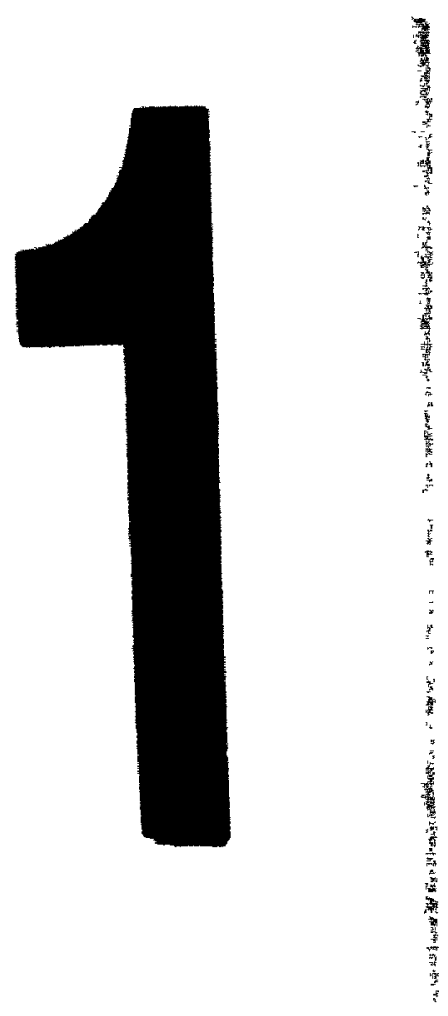

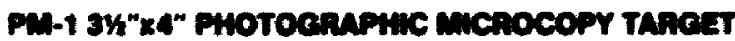

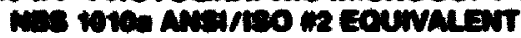

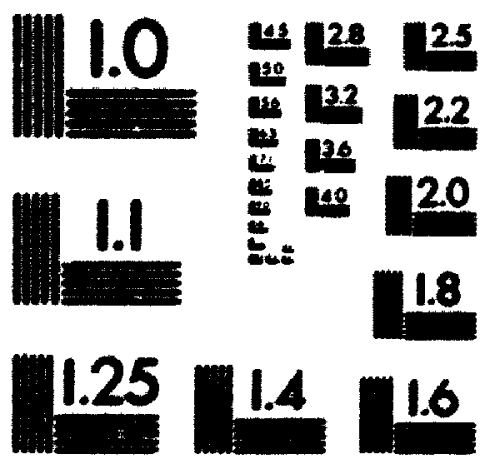

PRecranowem Resoumon tancets 
National Library

of Canada

Acquisitions and

Bibliographic Services Branch

395 Wethington Street

Otawa, Ontario

KiA ONA
Bibliotheque nationale

du Canada

Direction des acquisitions et

des services bibliographiques

395, ne Woltington

Ontawa (Ontano)

KIA ONA
Your liw votrecterence

Ou the Notre reference
NOTICE

The quality of this microform is heavily dependent upon the quallty of the original thesis submitted for microfilming. Every effort has been made to ensure the highest quality of reproduction possible.

If pages are missing, contact the university which granted the degree.

Some pages may have indistinct print especially if the original pages were typed with a poor typewriter ribbon or if the university sent us an inferior photocopy.
La qualite de cette microforme dépend grandement de la qualité de la these soumise au microfilmage. Nous avons tout fait pour assurer une qualité supérieure de reproduction.

S'll manque des pages, veuillez communiquer avec l'université quil a conféré le grade.

La qualite d'impression de certaines pages peut laisser à désirer, surtout si les pages originales ont ete dactylographiées à l'aide d'un ruban usé ou si l'université nous a fait parvenir une photocopie de qualit' inférieure.

La reproduction, méme partielle, de cette microforme est soumise à la Loi canadienne sur le droit d'auteur, SRC 1970, c. C-30, et ses amendements subséquents. 


\title{
Some Contributions to L-estimation in Linear Regression and Autoregression Models
}

\author{
by \\ Soroush Alimoradi, B.Sc., M.Sc. \\ A thesis submitted to \\ the Faculty of Graduate Studies and Research \\ in partial fulfillment of \\ the requirements for the degree of \\ Doctor of Philosophy \\ Department of Mathematics and Statistics \\ Ottawa-Carleton Institute of Mathematics and Statistics \\ Carleton University \\ Ottawa, Ontario \\ , 1995 \\ (C) Copyright \\ 1995, Soroush Alimoradi
}


Netionel Librery

of Ceneda

Acquistions and

Bibliographic Services Brench

305 Wumingon sineat

Oatum Orterio

RiAONA
Etbliotheque nationele

du Canada

Direction des acquisitions et

des senvices bibliographiques

395, ne Wollington

Otimave (Onterio)

KIA ONA vou the voire numence

Out the Norre nuturesce
The author has granted an irrevocable non-exclusive licence allowing the National Library of Canada to reproduce, loan, distribute or sell coples of his/her thesle by any means and in any form or format, making this thesis avallable to interested persons.
L'auteur a accordé une licence irrovocable of non exclusive permettant a la Bibliotheque nationale du Canada de reproduire, préter, distribuer ou vendre des copies de sa these de quelque maniere ot sous quelque forme que ce solt pour mettre des exemplaires de cette thdse a la disposition des personnes intéressées.

L'auteur conserve la proprióté du droit d'auteur qui protege sa these. Ni la these ni des oxtraits substantiels de colle-cl ne doivent stre imprimes ou autrement reprodults sane son autorisation. 
Nom Soroush. Alimoradi

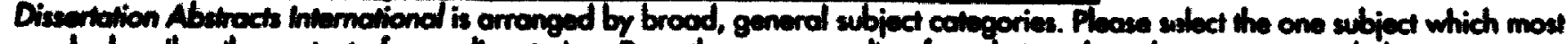
neorly deseribes the content of your diseartation. Enter the corresponding four-digit code in the speces provided.

Statistics
0141613 U.M.I strater

\section{Subind Conguies}

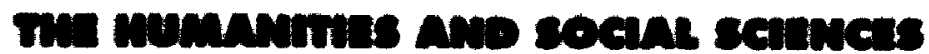
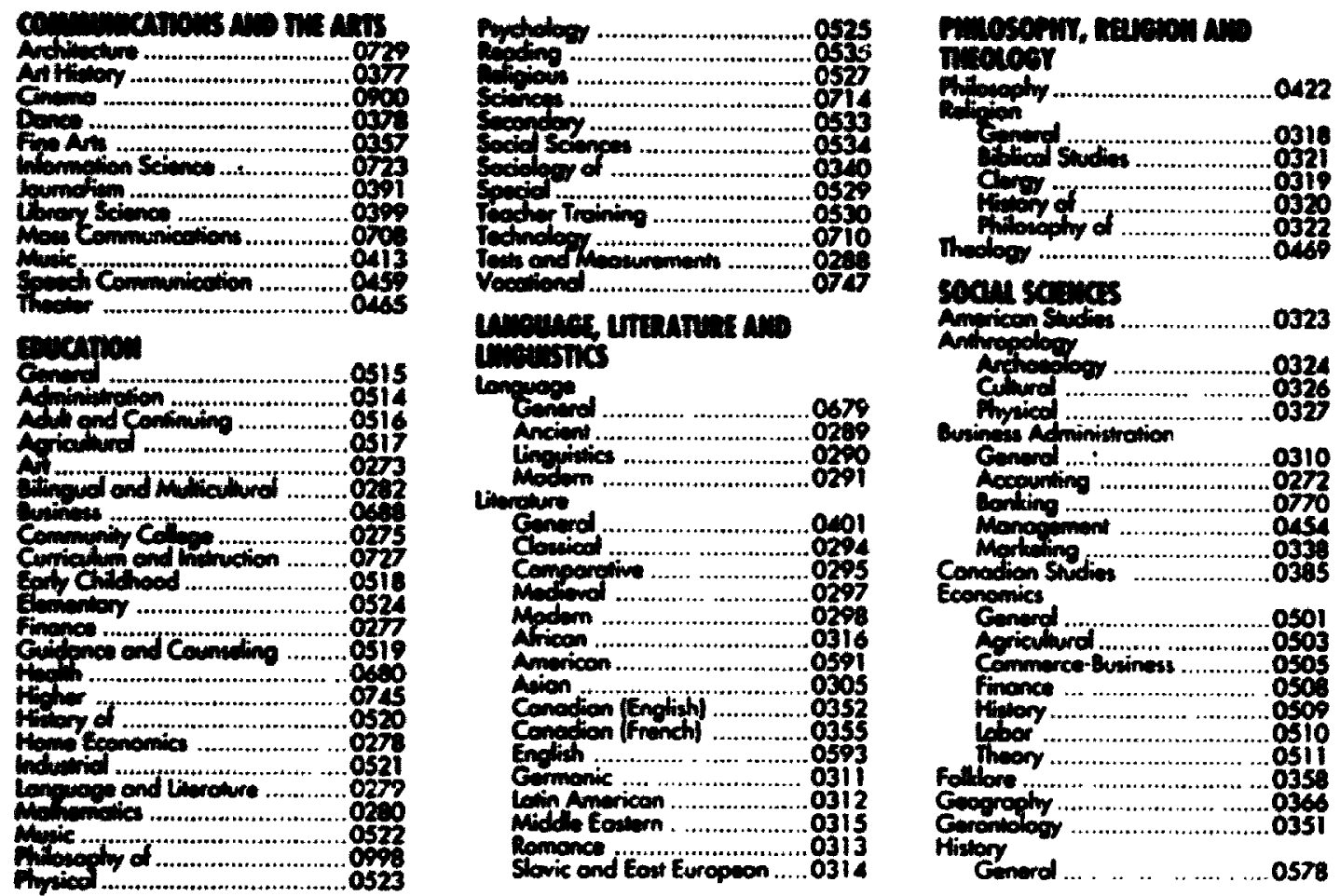

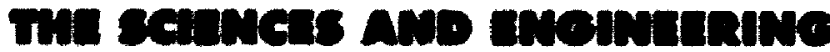

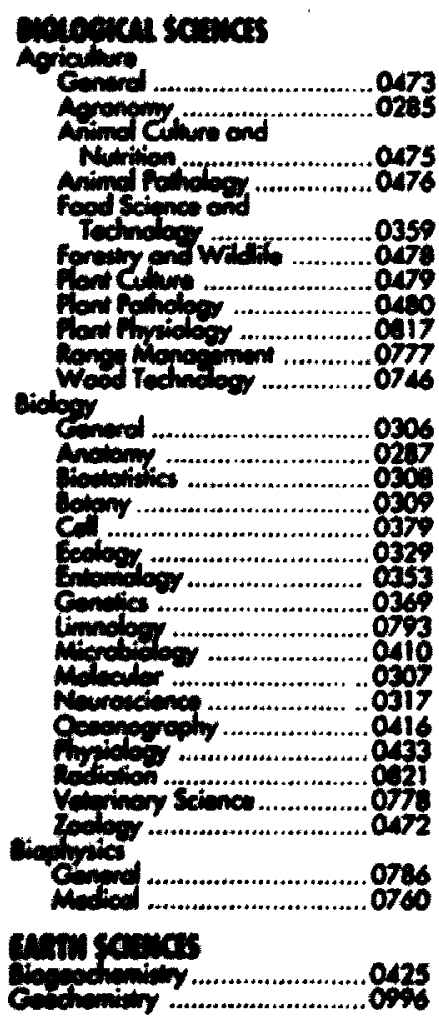

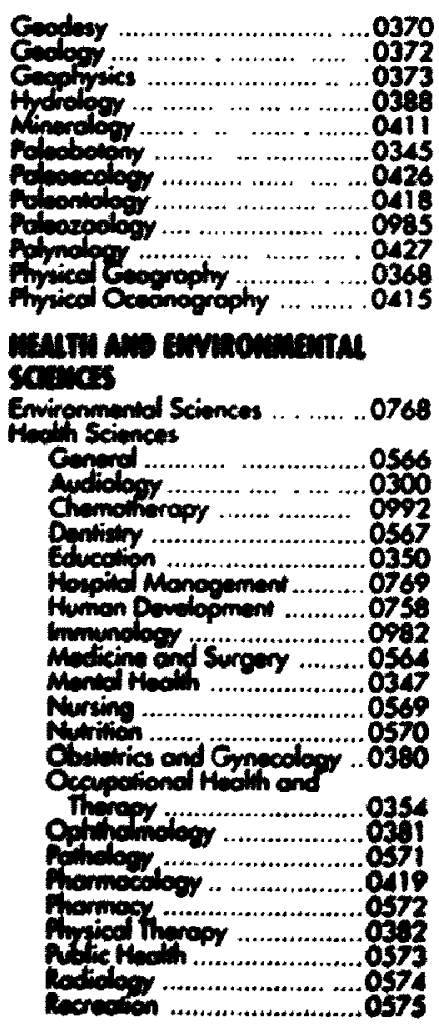

Q422

o318

310

(3)

Thelogr ....................................036

Animepelon

0323

0324

0327

0310

0272

04s:

0385

0501

0500

osit

0358

0578
Sean saings

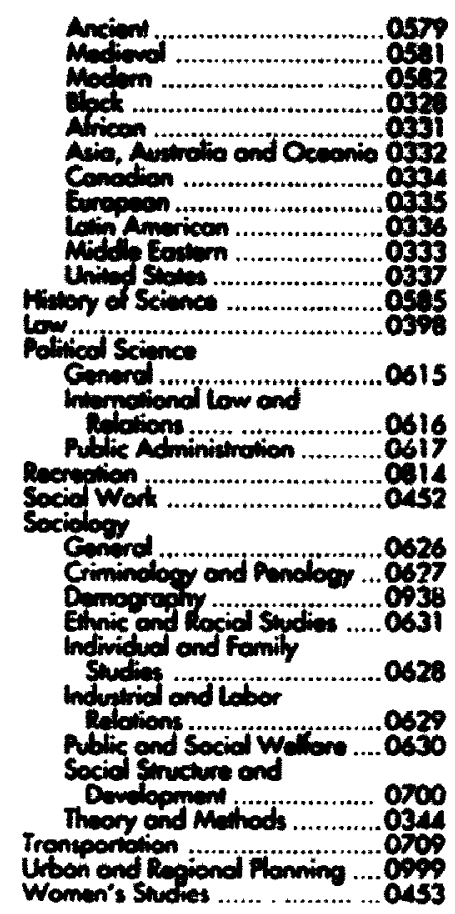

Spach Pothology . . . . .....8480 Toxicology
Hom Economics

Pursecu satios

Pure Sciunces

Chemisty

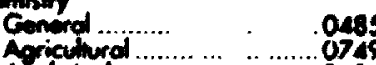

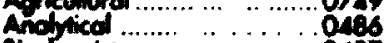

Inorgonic. ....................0489

Nuctoor .......................... . .

Orgonic ...............................0\%

Prived ...........................0494

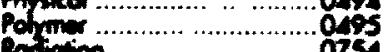

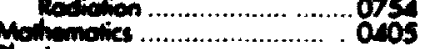

Physice

Cenerel .............................0\%

Acevilics..........

096

Alroghries

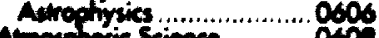

Ampir .........................074

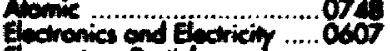

Eamentery Portidas and

Fiot Ento......

.0798

werler

Nectoor ....................................... OS10

Opics .............................0752

Solvition .............................858

sutitios

0463

Aritid seimoss

Apind Muchanic

g24
Enginouring $\ldots \ldots \ldots \ldots \ldots \ldots \ldots \ldots . .0537$

Arospace........................0538

Aoriculurd ... ... ................. 0538

Aulomptive .......................... 054

Chemical ............................0542

Cwit ……….................... 0543

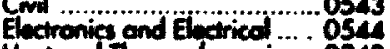

Hout ond thermodynomics ..0348

Thdredic ........................ 0545

Maring

Molriols science ...............0794

Muchonied .................... 0548

Metelurey ....................... 0743

Mining .......................... Oss

Pucker ..........................os52

Petrobum .....................0765

Sonitary ond Municipol .......0554

Sycem Science .....................0790

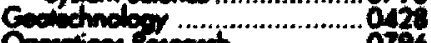

Gperdiom freverch .................. 079

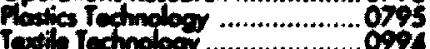

cromantery

amerd

.

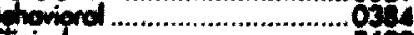

Ciniced ..............................087

meveriod...

Perwontity

myioleceol

protoblog

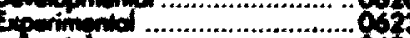

Pephonimites
Biechemistiny ...................0487 
The undersigned hereby recommend to The Faculty of Graduate Studies and Research acceptance of the thesis,

Some Contributions to L-estimation in Linear Regression and Autoregression Models submitted by

Soroush Alimoradi, B.Sc., M.Sc.

in partial fulfilment of the requirements for the degree of Doctor of Philosophy

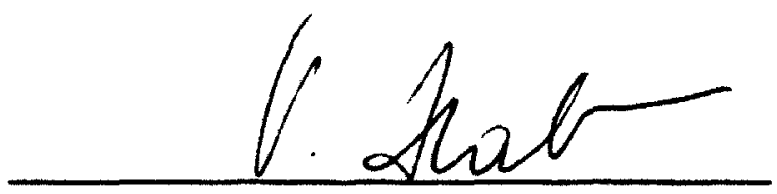

Professor V. Dab, Chair

Department of Mathematics and Statistics

Ces.ind Each

Professor A.K.Md.E. Saleh, Thesis Supervisor

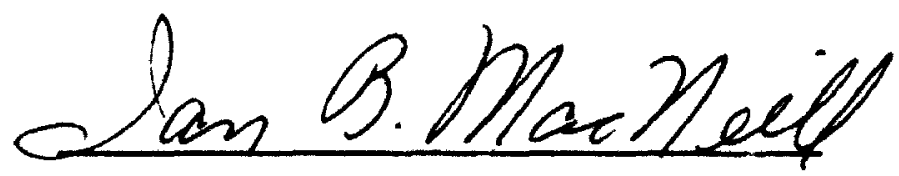

Professor lan B. MacNeill, External Examiner

Carleton University

1995 


\section{Abstract}

The theory of linear combinations (L-estimation) of order statistics/sample quantiles for the location model is well-known. This thesis considers the problem of L-estimation in linear and autoregression models.

First, we extend L-estimation methods for the regression parameters in a linear model based on a few selected regression quantiles and deal with the question of optimum regression quantiles. We also provide tables of optimum spacings, coefficients of $A B L U E$ and the quantities required to calculate the asymptotic efficiencies of these simplified L-estimators with $t$ and the logistic errors. Second, we consider Bahadur type representation of the regression quantiles and the trimined LSE under less restrictive conditions than that of Jurečková (1984) and we prove the asymptotic normality of these estimators. Third, we consider the estimation of the regression parameters under some uncertain prior sub-space restriction leading to the combination of two diverse arees, namely, robust estimation and shrinkage estimation via preliminary test approach.

Though, there has been some, progress on L-estimation for the linear model, relatively little progress has been made with the most popular time series model in application, namely, the $\operatorname{AR}(p)$-model. We develop the autoregression quantiles (ARQ) approach due to Koul and Saleh (1995) in order to deal with some aspects of L-estimation of the parameters of the $p^{\text {th }}$ order autoregression model. First, we 
consider the problem of L-estimation of the parameters of the AR(p)-model based on a few selected ARQ's. We also provide tables of optimum spacings, coefficients of ABLUE and the quantities required to calculate the asymptotic efficiencies of these simplified L-estimators with $t$ and the logistic errors. Second, we consider Bahadur type representation of the autoregression quantiles and the trimmed least square estimators (TLES) under the same conditions as we have done for regression model. Finally, we consider the preliminary test approach to shrinkage estimation to improve the robustified L-estimators in a sub-hypothesis problem. 


\section{Acknowledgements}

I am very grateful to my thesis supervisor Dr. A.K.Md. Ehsanes Saleh who introduced me to the area of regression quantiles and L-estimation. Without his constant encouragement and insight into the subject matter of the thesis, it mo not have come to reality. I will be ever thankful to him for his supervision and guidance in my ph.D program.

As well, thanks to Dr. Hira L. Koul of Michigan State University, USA for his very helpful and constructive discussion on the proofs of some theorems when he visited the department during summer 1995.

I would like to acknowledge Dr. S. Mills for her time she put to read my thesis carefully and pointing out editorial changes and also to Dr. A. Bose for his time to read my thesis.

I am also grateful to the Islamic Republic of Iran for scholarship to pursue my study in Canada.

Last, but not least, I grateful to my wife Mrs. N. Monzavi who helped me in typing of my thesis. I owe my achievement to her, my children Saied and Fatemeh, my parents and my parents in-law for their love and support. 


\section{Contents}

1 Introduction 1

2 Location and Scale Model 7

2.1 Introduction $\ldots \ldots \ldots \ldots \ldots \ldots \ldots \ldots \ldots \ldots$

2.2 Estimation of location and scale parameters based on order statistics 7

2.3 Estimation of location and scale parameters based on sample quantiles 10

2.4 Trimmed Least-Squared Estimation of Location Parameter And Its

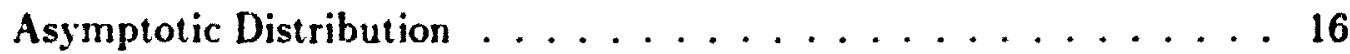

3 Regression Model $\quad 37$

3.1 Introduction $\ldots \ldots \ldots \ldots \ldots \ldots \ldots \ldots \ldots \ldots \ldots \ldots$

3.2 Regression Quantiles and Their Properties . . . . . . . 38

3.3 L-estimation of the parameters of a linear model based on a few selected regression quantiles with known error distributions. . . . . 43

3.3.1 Joint Estimation of Regression and Scale Parameters . . . . 44

3.3.2 Estimation of Conditional Quantile Function . . . . . . . 53

3.3.3 Estimating functions of parameters $\ldots \ldots \ldots \ldots \ldots$

3.4 Trimmed Least-Squared Estimation of Regression Parameters And Its Asymptotic Distribution $\ldots \ldots \ldots \ldots \ldots$ 
3.5 Trimmed Estimation of Regression Parameters Under Uncertain Prior

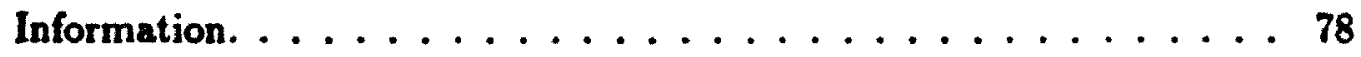

3.5.1 Five Trimmed Estimators . . . . . . . . . . . 80

3.5.2 Asymptotic Distributional Risks . . . . . . . . . . . 81

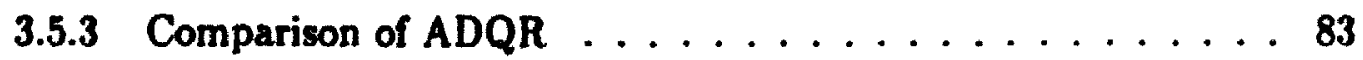

3.5.4 Conclusion . . . . . . . . . . . . . 87

4 Autoregression Model 89

4.1 Introduction . . . . . . . . . . . . . . 89

4.2 Autoregression Quantiles . . . . . . . . . . . . . . 90

4.3 L-estimation of the parameters of a autoregression model based on a few selected autoregression quantile . . . . . . . . . . 94

4.3.1 Joint Estimation of Autoregression and Scale Parameters . . 95

4.4 Trimmed Least Squares Estimation of AR(p) Parameters . . . . . 109

4.5 Estimation of Parameters Under Uncertain Prior Information. . . . 120

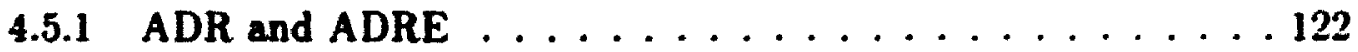

$\begin{array}{ll}\text { A t-dist. (location and scale parameters) } & 127\end{array}$

A t-Dist. (Regression Parameters, $p=1$ ) 135

A t-Dist. (Regression Parameters, $p=2$ ) 143

A t-Dist. (Regression Parameters, $p=3$ ) 151

A Logistic Distribution Table 159 


\section{Chapter 1}

\section{Introduction}

In the nineteenth century. linear combination of order statistics (the so called Lestimators) were in common use especially in astronomical calculations. Sometimes estimators were calculated by dropping smaller or larger values or both from the set of all observations giving the concept of trimming of oufliers. L-estimators, naturally, are an integral part of robust estimation (i.e. estimators which are not sensitive to small deviation from model distribution) of location parameter. In the case of location model sample mean has minimum variance in the class of unbiased estimators when the errors are normal and has also asymptotic efficiency of one. But this estimator has zero efficiency for the Cauchy distribution. Looking for alternative estimators which may be robust opposed to the sample mean has been apparent since the eighteenth century. There are three classes of estimator in the location model which are commonly used as robust estimators, namely, maximum likelihood type estimators (M-estimators), estimators based on ranks (R-estimators) and linear combination of order statistics (L-estimators). We describe them now. 
(i) M-estimators: Consider the location model

$$
Y_{i}=B_{0}+Z_{i} \quad ; \quad Z_{i} \in \mathbb{R}, \quad i=1, \ldots, n
$$

where $Z_{i}^{\prime} s, i=1, \ldots, n$ are $i . i . d$ with c.d.f. $F_{0}$. The M-estimator of $\beta_{0}$ is any solution of minimization problem

$$
\sum_{i} \rho\left(Y_{i}-b\right)=\min ; \quad b \in \mathbb{R}
$$

where $\rho$ is an arbitrary function, or solve the pseudo-likelihood equations with chosen ४-functions

$$
\sum_{i} \psi\left(Y_{i}-b\right)=0 .
$$

If $\rho$ is convex and $\psi^{\prime}=\rho^{\prime}$ these definitions coincide. Note that if we choose $\rho\left(Y_{i}-b\right)=-\log f_{0}\left(Y_{i}^{\prime}-b\right)$, it gives the ordinary MLE with $\psi=f_{0}^{\prime} / f_{0}$. The asymptotic properties of this kind of estimators are given by Huber (1981).

(ii) R-estimators: A robust estimator of location may be derived from a test statistic that is distribution-free under an appropriate null hypothesis about $\beta_{0}$. This technique was first proposed by Hodges and Lehmann (1963). A test can be carried cut based on one or two samples. In the case of one sample, let $Y_{3} \ldots, Y_{n}$ be a random sample from a continuou; distribution with $c . d . f F_{0}\left(y-\beta_{0}\right)$ when $F_{0}(\cdot)$ is symmetric about zero. Assume that $H_{0}: \beta_{0}=0$ vs $H_{1}: \beta_{0}>0$ and let $V\left(Y_{1}, \ldots, Y_{n}\right)$ be a test statistic which satisfies the following conditions:

i) $H_{0}$ is rejected when $V\left(Y_{1}, \ldots, Y_{n}\right)$ is large.

ii) For each $\left(y_{1}, \ldots, y_{n}\right), V\left(y_{1}+h, \ldots, y_{n}+h\right)$ is a nondecreasing function of $h$.

iii) Under $H_{0}$, the distribution of $V\left(Y_{1}, \ldots, Y_{n}\right)$ is symmetric about a value $\xi$ for every $F_{0}(\cdot)$ which is continuous and symmetric about 0 .

Now, we motivate a Hodges-Lehmann estimator of $\beta_{0}$ as follows. The random varjables $Y_{1}-\beta_{0}, \ldots, Y_{n}-\beta_{0}$ are independent with symmetric identical distribution. Thus, it is desirable to find an estimator of $\beta_{0}$, say $\hat{\beta}_{0}$, such that $Y_{1}-\hat{\beta}_{0}, \ldots, Y_{n}-\hat{\beta}_{0}$ 
is symmetrically distributed about 0 . The Hodges-Lehmann estimator of $\beta_{0}$ based on a test statistic $V\left(Y_{1}, \ldots, Y_{n}\right)$ satisfying (i), (ii) and (iii) is given by

$$
\hat{\beta}_{0}=\frac{\beta_{0}^{*}+\beta_{0}^{*}}{2},
$$

where

$$
\begin{aligned}
\beta_{0}^{*} & =\sup \left\{\beta_{0}: V\left(Y_{1}-\beta_{0}, \ldots, Y_{n}-\beta_{0}\right)>\xi\right\} . \\
\text { and } \beta_{0}^{* *} & =\sup \left\{\zeta_{0}: V\left(Y_{1}-\beta_{0}, \ldots, Y_{n}-\beta_{0}\right)<\xi\right\} .
\end{aligned}
$$

More details about this estimator and other R-estimators t...sed on ome sample with asymptotic properties can be seen in Randles and Wolfe (1979). Huber (1981) and Puri and Sen (1985)among others.

(iii) L-estimator- The mathematical theory of L-estimation of location and scale parameters for known distributions began with the work of Ogawa (1951) and Lloyd (1952). Lloyd's (1952) work pertains to best linear unbiased estimation (BLLE) based on order statistics in finite samples while Ogawa's (1951) work is based on the asymptotic distributions of several sample quantiles in large samples due to Mosteller (1946). This paved the way for the developments regarding the asymptotically best linear unbiased estimation (ABLCE) of the location and scale parameters from known distributions. These simplified L-estimators are precursors of the socalled Gastwirth's (1966) robust estimator of the location parameter of symmetric distributions.

Let $Y_{(1)}, \ldots, Y_{(n)}$ denote the order statistics of the sample $Y_{1} \ldots, Y_{n}$. Then a sequence of estimators whose elements are linear combinations of order statistics take the form

$$
\dot{\beta}_{0 n}=n^{-1} \sum_{i=1}^{n} J\left(\frac{i}{n+1}\right) Y_{(r)}
$$

for some function $J(\cdot)$ on $(0,1)$, and are called L-estimator with continuous weight function. Typically, $J(\cdot)$ is chosen to satisfy $J(t)=J(1-t)$ and $\int_{0}^{1} J(t) d t=1$. 
An asymptotically efficient L-estimator of location parameter $\beta_{0}$ in $F_{0}\left(z-\beta_{0}\right)$ is obtained by setting

$$
J\left(F_{0}(z)\right)=\frac{\left[\log f_{0}(z)\right]^{\prime \prime}}{I\left(F_{0}\right)}
$$

where $I\left(F_{0}\right)$ is the Fisher's information number. Readers may refer to Jung (1955), Chernoff, Gastwirth and Johns (1967), and Stigler (1974) among others. An Lestimator is said to be robust only if for some $\lambda \in(0,1 / 2], J(t)$ has the support $[\lambda, 1-\lambda]$. Examples of such robust estimators are the quick and dirty or inefficient estimators of Mosteller (1946) and Gastwirth's (1966) L-estimators. Undoubtedly, the most important class of L-estimators is the class of trimmed means which have the weight function

$$
J(t)=\frac{1}{1-2 \lambda}, \quad t \in[\lambda, 1-\lambda] .
$$

The idea of such a trimmed estimator is due to Tukey (1960). The asymptotic behavior of this estimator is treated in Stigler (1973). These subjects are again treated in chapter 2.

Analogues of M-estimators in the linear multiple regression model carry over from the location model virtually intact (see for example, Huber (1981)), though the problem of invariance under reparametrization of the design matrix remains a problem. As regards the R-estimator of the regression coefficients, the problem of alignment crops in the estimators of parameters in a sub-hypothesis problem (see Puri and Sen (1985)). As regards L-estimators of parameters in the linear model, the definition of order statistic/quantile became the nagging problem. However, Bickel (1973) extended the idea of linear combination of order statistics by ordering the residuals based on a preliminary estimator such as a least-square estimator. These estimators may be computed by iter 'ively reweighted least squares but are complicated and have the disadvantage of being not invariant to reparametrization of design matrix.

An alternative approach to L-estimators in the linear model is due to the well- 
accepted definition of regression quantiles and their properties by Koenker and Bassett (1978) which extends the definition of sample quantiles in location model to the linear model. The idea begins with the observation that the ordinary sample quantiles/order statistics of the location model, may be regarded as the M-estimations with $\rho$-function

$$
\rho_{\lambda}(x)=\left\{\begin{array}{cc}
\lambda x, & x \geq 0 \\
(\lambda-1) x, & x<0
\end{array}\right.
$$

where $\lambda \in(0,1)$. Then, it is straight forward to see that this $\rho$-function in the linear model yields a p-dimensional analogue of the sample quantiles. The median case $(\lambda=1 / 2)$ is the well-known least-absolute error (LAE) regression. This concept seems to provide a reasonable basis for the construction of robust L-estimators of regression parameters. The asymptotic behavior of the $p$ dimensional regression quantiles is strikingly similar to the behavior of the ordinary sample quantiles. Later on Ruppert and Carroll (1980) studied the trimmed least squares estimators (TLSE) based on regression quantiles. They derived the Bahadur-type representation of regression quantiles up to the order $o_{P}\left(n^{-1 / 2}\right)$ as well as the asymptotic distribution of the trimmed LSE when the design matrix satisfies the following conditious on the design elements:

$$
x_{i 1}=1 \quad i=1, \ldots, n ; \quad \sum_{i=1}^{n} x_{i j}=0 \quad j=2, \ldots, p+1 .
$$

Jurečková (1984) considered the same problem under general conditions on the elements of the design matrix. She also derived the Bahadur-type representation for regression quantiles and linear representation of the trimmed LSE up to the order $O_{P}\left(n^{-3 / 4}\right)$ as well as asymptotic distribution of TLSE, when the error distribution function $F$ is absolutely continuous and its second derivative is bounded. For the design matrix she considered conditions such as the average of the fourth power of design matrix elements should be bounded as $\mathbf{n}$ goes to infinity.

The main contribution on L-estimation begins with Chapter 3 on the linear re- 
gression model.First, we extend L-estimation methods for the regression parameters in a linear model based on a few selected regression quantiles and deal with the question of optimum regression quantiles. We also provide tables of optimum spacings, coefficients of ABLUE and the quantities required to calculate the asymptotic efficiencies of these simplified L-estimators with $t$ and the logistic errors. Second, we consider Bahadur type representation of the regression quantiles and the trimmed LSE under less restrictive conditions than that of Jurečková (1984) and we prove the asymptotic normality of these estimators. Third, we consider the estimation of the regression parameters under some uncertain prior sub-space restriction leading to the combination of two diverse areas, namely, robust estimation and shrinkage estimation via preliminary test approach.

Though, there has been some progress on L-estimation for the linear model, relatively little progress has been made with the most popular time series model in application, namely, the $A R(p)$-model.

In the final chapter 4, we develop the autoregression quantiles (ARQ) approach due to Koul and Saleh (1995) in order to deal with some aspects of L-estimation of the parameters of the $p^{\text {th }}$ order autoregression model. First, we consider the problem of L-estimation of the parameters of the AR(p)-model based on a few selected ARQ's. We also provide tables of optimum spacings, coefficients of ABLUE and the quantities required to calculate the asymptotic efficiencies of these simplified L-estimators with $t$ and the logistic errors. Second, we consider Bahadur type rpresentation of the autoregression quantiles and the trimmed least square estimators (TLES) under the same conditions as we have done for regression model. Finally, we consider the preliminary test approach to shrinkage estimation to improve the robustified L-estimators in a sub-hypothesis problem. 


\section{Chapter 2}

\section{Location and Scale Model}

\subsection{Introduction}

The object of this chapter is to review the developments of the estimation of locationscaie parameters of a distribution based on order statistics and a few selected sample quantiles leading to the so-called the theory of L-estimation. Section 2.2 contains the preliminary developments of L-estimation theory based on order statistics due to Lloyd (1952). In section 2.3, we present the theory of L-estimation based on a few sample quantiles due to Ogawa (1951). This section contains some new tables of optimum spacings and related coefficients for the L-estimation of location and scale parameters for the class of $t$-distributions. The last section (2.4) contains the trimmed least-squares estimator for location parameter. These ideas are treated in full detail which will be continued in later chapters with linear and autoregressive models.

\subsection{Estimation of location and scale parameters based on order statistics}

Consider the location model

$$
Y_{j}=\beta_{0}+\sigma Z, \quad j=1, \cdots, n
$$


where $Z_{1}, Z_{2}, \ldots, Z_{n}$ are i.i.d. r.v's with continuous cdf $F_{0}(z)$ (and $p d f f_{0}(z)$ ), $\left(\beta_{0}, \sigma\right)^{\prime}$ stands for the location and scale parameters of the distribution. Let $\boldsymbol{Y}_{(\cdot)}=$ $\left(Y_{(1)}, \cdots, Y_{(n)}\right)^{\prime}$ be the order statistic vector corresponding to $Y=\left(Y_{1}, \cdots, Y_{n}\right)^{\prime}$. Similarly, let $Z_{(\cdot)}=\left(Z_{(1)}, \cdots, Z_{(n)}\right)^{\prime}$ be the order statistic vector corresponding to $Z=\left(Z_{1}, \cdots, Z_{n}\right)^{\prime}$. Clearly,

$$
Y_{(\cdot)}=\beta_{0} 1_{n}+\sigma Z_{(\cdot)}, \quad 1_{n}=(1, \cdots, 1)^{\prime} .
$$

Further,

$$
E Y_{(\cdot)}=E\left(Y_{(1)}, \cdots, Y_{(n)}\right)^{\prime}=\beta_{0} 1_{n}+\sigma E Z_{(\cdot)}
$$

and

$$
\operatorname{cov}\left(Y_{(-)} Y_{(.)}^{\prime}\right)=\sigma^{2} \operatorname{cov}\left(Z_{(\cdot)} Z_{(\cdot)}^{\prime}\right)
$$

Since $F_{0}$ is known, $E Z_{(\cdot)}=\alpha_{(\cdot)}$ and $\operatorname{cov}\left(Z_{(\cdot)} Z_{(\cdot)}^{\prime}\right)=V$ are completely known. Thus, Lloyd (1952) proposed the linear (L-) estimation of $\left(\beta_{0}, \sigma\right)^{\prime}$ based on $Y_{(\cdot)}$ by minimization of the quadratic form

$$
\left(Y_{(\cdot)}-\beta_{0} 1_{n}-\sigma \alpha_{(\cdot)}\right)^{\prime} V^{-1}\left(Y_{(\cdot)}-\beta_{0} 1_{n}-\sigma \alpha_{(\cdot)}\right)
$$

with respert to $\beta_{0}$ and $\sigma$. The minimization process leads to the normal equations

$$
1_{n}^{\prime} V^{-1}\left(Y_{(\cdot)}-\beta_{0} 1_{n}-\sigma \alpha_{(\cdot)}\right)=0
$$

and

$$
\alpha_{(\cdot)}^{\prime} V^{-1}\left(Y_{(\cdot)}-\beta_{0} 1_{n}-\sigma \alpha_{(\cdot)}\right)=0
$$

This results in solving for $\beta_{0}$ and $\sigma$ from the equations

$$
\begin{aligned}
& \hat{\beta}_{0} d_{11}+\hat{\sigma} d_{12}=M_{1} \\
& \hat{\beta}_{0} d_{12}+\hat{\sigma} d_{22}=M_{2}
\end{aligned}
$$

where

$$
\begin{gathered}
d_{11}=1_{n}^{\prime} V^{-1} 1_{n}, d_{22}=\alpha_{(,)}^{\prime} V^{-1} \alpha_{(\cdot)}, d_{12}=\alpha_{(,)}^{\prime} V^{-1} 1_{n}, \\
M_{1}=1_{n}^{\prime} V^{-1} Y_{(\cdot)}, M_{2}=\alpha_{(\cdot)}^{\prime} V^{-1} Y_{(\cdot)} \text { and } D=d_{11} d_{22}-d_{12}^{2} .
\end{gathered}
$$


The explicit expression for $\hat{\beta}_{0}$ and $\hat{\sigma}$ are given by

$$
\hat{\beta}_{0}=\frac{1}{D}\left(M_{1} d_{22}-M_{2} d_{12}\right)
$$

and

$$
\dot{\sigma}=\frac{1}{D}\left(M_{2} d_{11}-M_{1} d_{12}\right)
$$

respectively. The covariance matrix for $\left(\hat{\beta}_{0}, \hat{\sigma}\right)^{\prime}$ is then given by

$$
\operatorname{Cov}\left(\dot{\beta}_{0}, \hat{\sigma}\right)=\frac{\sigma^{2}}{D}\left(\begin{array}{cc}
d_{22} & -d_{12} \\
-d_{12} & d_{11}
\end{array}\right) .
$$

The estimators $\hat{\beta}_{0}$ and $\hat{\sigma}$ are best linear unbiased estimators (BI,UEs) of $\beta_{0}$ and $\sigma$ respectively.

In the fifties and sixties many researchers such as Sarhan, Greenberg, Ogawa, Kulldorff and Saleh investigated properties of the above estimators based on a few order statistics. The studies covered uniform, exponential, double-exponential, logistic and normal distributions to name a few. The results of such studies have been documented in the monograph Contributions to Order Statistics by Sarhan and Greenberg (1962), Order Statistics by David (1981) and Order Statistics and Inference by Balakrishnan and Cohen (1991) among others. The methodology of the BLUE of $\left(\beta_{0}, \sigma\right)$ remains the same based on $k$ order statistics except that the observation vector $Y_{(\cdot)}$ of $n$-dimension is taken as

$$
Y_{(.)}^{k}=\left(Y_{\left(n_{1}\right)}, \cdots, Y_{\left(n_{k}\right)}\right)^{\prime}
$$

where $Y_{\left(n_{1}\right)}, \cdots, Y_{\left(n_{k}\right)}$ are the selected order statistics (with ranks $n_{1}, \cdots, n_{k}$ ) from $\boldsymbol{Y}_{(\cdot)}=\left(Y_{(1)}, \cdots, Y_{(n)}\right)^{\prime}$ and correspondingly $\boldsymbol{V}$ is changed to $k \times k$ matrix $V^{(\cdot)}=$ $\operatorname{cov}\left(Z_{(\cdot)}^{(k)} Z_{(\cdot)}^{(k)^{\prime}}\right), D$ is changed to $D^{(k)}$, and $d_{i j} \rightarrow d_{i j}^{(k)}$, for finite sample situations. The problem of optimum order statistics for a given $k(2 \leq k \leq n)$ arose and was solved specially for the exponential distribution by Sarhan and Greenberg (1962), Kulldorff (1964) and Saleh (1967) in complete and type Il censored samples. The 
relative efficiency (R.E.) of BLUEs' based on $k(2 \leq k \leq n)$ order statistics compared to the BLUEs' based on all observations may be written as

$$
R . E .\left(B L U E_{(k)} ; B L U E_{(n)}\right)=\frac{D^{(k)}}{D}=\frac{d_{11}^{(k)} d_{22}^{(k)}-\left(d_{12}^{(k)}\right)^{2}}{d_{11} d_{22}-d_{12}^{2}}
$$

\subsection{Estimation of location and scale parameters based on sample quantiles}

Mosteller (1946) proposed the use of so-called inefficient statistics based on a few sample quantiles as quick and dirty substitutes for more conventional estinators. Mosteller's idea was quickly extended by $O_{\text {gawa }}(1951)$ and provided the asymptotically best linear unbiased estimators (ABLUEs) of $\beta_{0}$ and $\sigma$ based on $k(2 \leq k<n)$ selected sample quantiles. Now we discuss this area of research which expanded during the fifties and sixties having implications in robust estimation of location parameters. First, we consider various definitions.

Definition 2.3.1 Let $Z$ be a random variable with adf $F_{0}(z)$. Then, $Q_{0}(\lambda)$ is called the population quantile-function of order $\lambda(0<\lambda<1)$ given by

$$
Q_{0}(\lambda)=\inf \left\{z: F_{0}(z) \geq \lambda\right\}=F_{0}^{-1}(\lambda)
$$

Definition 2.3.2 Let $Z$ be a random variable with $c d f F_{0}(z)$ and $p d f f_{0}(z)$. The density evaluated at $Q_{0}(\lambda)$, namely, $f_{0}\left(Q_{0}(\lambda)\right)$ is called the density-quantile function and is denoted by $q_{0}(\lambda)$.

Some quantile-functions and density-quantile function are given below. 
Table 2.1: Quantile functions and density quantile functions

\begin{tabular}{|l|c|c|c|} 
Dist. & Densities & $u=Q_{0}(\lambda)$ & $q_{0}(\lambda)$ \\
\hline Normal & $(2 \pi)^{-1 / 2} \exp \left(-z^{2} / 2\right)$ & $\Phi^{-1}(\lambda)$ & $(2 \pi)^{-1 / 2} \exp \left\{-\frac{1}{2}\left[\Phi^{-1}(\lambda)\right]^{2}\right\}$ \\
Cauchy & $\pi^{-1}\left(1+z^{2}\right)^{-1}$ & $\tan \left[\pi\left(\lambda-\frac{1}{2}\right)\right]$ & $\pi^{-1} \sin ^{2}(\pi \lambda)$ \\
Logistic & $e^{\frac{-\pi}{\sqrt{3}} /\left(1+e^{-\frac{1}{2}}\right)^{2}}$ & $\frac{\sqrt{3}}{\pi} \ln \left(\frac{\lambda}{1-\lambda}\right)$ & $\lambda(1-\lambda)$ \\
Log Nor. & $(2 \pi)^{-1 / 2} z^{-1} e^{-\frac{1}{2}(\ln z)^{2}}$ & $\exp \left[\Phi^{-1}(\lambda)\right]$ & $(2 \pi)^{\frac{1}{2}} e^{\left[\left(-\frac{1}{2}\left(\phi^{-1}(\lambda)\right)^{2}\right\}-\psi^{-1}(\lambda)\right]}$ \\
Double Exp. & $\frac{1}{2} e^{-|z|}$ & $\ln (2 \lambda), \lambda<\frac{1}{2}$ & $\left\{\begin{array}{cc}\lambda & \text { if } \lambda<\frac{1}{2} \\
1-\lambda & \text { if } \lambda>\frac{1}{2}\end{array}\right.$ \\
\hline
\end{tabular}

Let $Z_{1}, Z_{2}, \cdots, Z_{n}$ be a random sample of size $n$ from the common $\operatorname{cdf} F_{0}(z)$. Let $F_{0 n}(z)$ defined by

$$
F_{0 n}(z)=n^{-1}\left(\# Z_{i} \leq z\right)=n^{-1} \sum_{i=1}^{n} I\left(Z_{i} \leq z\right)
$$

be the empirical $c d f$ corresponding to $F_{0}(z)$. Then

$$
\bar{Q}_{0_{n}}(\lambda)=\inf \left\{z: F_{0_{n}}(z) \geq \lambda\right\} \equiv F_{0 n}^{-1}(\lambda)
$$

is the sample-quantile of order $\lambda(0<\lambda<1)$. Another popular way is to write

$$
\bar{Q}_{O_{n}}(\lambda)=Z_{(j)}, \quad \text { if } \frac{j-1}{n} \leq \lambda<\frac{i}{n} .
$$

for $j=1,2, \cdots, n$. This is a piecewise constant function over the interval $[0,1]$ and $Z_{(j)}$ stands for the order statistic with rank $j(1 \leq j \leq n)$. More commonly, one writes

$$
\begin{aligned}
\bar{Q}_{0 n}(\lambda) & =Z_{([n \lambda]+1)} \quad \text { if } n \lambda \text { is not an integer } \\
& =Z_{(n \lambda)} \quad \text { if } n \lambda \text { is an integer }
\end{aligned}
$$

However, a more general definition of sample quantile of order $\lambda$ for a location model

$$
Y_{i}=\beta_{0}+Z, \quad j=1, \cdots, n
$$


is the solution of the following minimization problem:

$$
\min _{b \in \mathbb{R}}\left[\sum_{t \in\left\{t: y_{t} \geq b\right\}} \lambda\left|y_{t}-b\right|+\sum_{t \in\left\{t: y_{t}<b\right\}}(1-\lambda)\left|y_{t}-b\right|\right] .
$$

This criterion was alluded to by Ferguson (1967) in an exercise and extended by Koenker and Bassett (1978). The case of the median is achieved when $\lambda=1 / 2$ which is well-known as the solution of the minimization problem:

$$
\min _{b \in \mathbb{R}}\left[\sum_{t=1}^{n}\left|y_{t}-b\right|\right]
$$

Sample quantiles in the location model are either identified with a single order statistic or a group of order statistics from the observed sample, for example in the case of the median from an even sample, they are identified with a closed interval between two order statistics and some combination of these two order statistics, say, the average. In fact if $F_{0}$ is continuous, then the solution of (2.22) is unique if and only if

$$
\lambda-1<\sum_{t \neq h}\left\{1 / 2-1 / 2 \operatorname{sign}\left(y_{t}-y_{h}\right)-\lambda\right\}<\lambda .
$$

See for example Bassett and Koenker (1978). The expression in the bracket takes the value $-\lambda$ if $y_{t}>y_{h}$ and $1-\lambda$ if $y_{t}<y_{h}$ so that (2.24) reduces to the requirement that the number of $y_{t}^{\prime} s$ less than $y_{h}$ be strictly between $n \lambda-1$ and $n \lambda$. This demands that $n \lambda$ be non-integral. In the case of the median the requirement boils down to $n$ being odd.

It is easy to verify that the sample quantiles satisfy the location and scale equivariance properties. Now we consider the asymptotic distribution of several sample quantiles, a result due to Mosteller (1946).

Theorem 2.3.3 Let $Y_{1}, Y_{2}, \cdots, Y_{n}$ be a random sample corresponding to the random variable with cdf $F_{0}\left(\frac{1-\beta_{0}}{-}\right)$ and pdf $\frac{1}{6} f_{0}\left(\stackrel{\underline{y}-\beta_{0}}{-}\right)$ continuous and positive at $Q\left(\lambda_{i}\right)=$ $\beta_{0}+\sigma Q_{0}\left(\lambda_{i}\right), i=1, \cdots, k(2 \leq k$ klen $)$ where $0<\lambda_{1}<\lambda_{2}<\cdots<\lambda_{k}<1$ are prefixed real numbers in $(0,1)$. Define $n_{j}=n \lambda_{j}+o\left(n^{-1 / 2}\right), j=1,2, \cdots, k$. Then,

$$
n^{1 / 2}\left(Y_{\left[n \lambda_{1}\right]+1}-Q\left(\lambda_{1}\right), \cdots, Y_{\left[n \lambda_{k}\right]+1}-Q\left(\lambda_{k}\right)\right)^{\prime} \stackrel{D}{\rightarrow} \mathcal{N}_{k}\left(0, \sigma^{2} \Omega_{k \times k}\right)
$$


whete

$$
\boldsymbol{\Omega}_{k \times k}=\left(\left(\frac{\lambda_{i} \wedge \lambda_{j}-\lambda_{i} \lambda_{j}}{q_{0}\left(\lambda_{i}\right) q_{0}\left(\lambda_{j}\right)}\right)\right)
$$

See Sen and Singer (1993) section 4.3 for the proof.

Ogawa (1951) formulated the asymptotically best linear unbiased estimators (ABLUEs) of $\beta_{0}$ and $\sigma$ based on $k(2 \leq k \leq n)$ selected sample quantiles using Mosteller's work. Thus, he minimized the quadratic form

$$
\left(Y_{(\cdot)}-\beta_{0} 1_{k}-\sigma Q_{0}(\lambda)\right)^{\prime} \Omega^{-1}\left(Y_{(\cdot)}-\beta_{0} 1_{k}-\sigma Q_{0}(\lambda)\right)
$$

with respect to $\beta_{0}$ and $\sigma$. Here, we have

$$
\begin{aligned}
Y_{(\cdot)} & =\left(Y_{\left[n \lambda_{1}\right]+1}, \cdots, Y_{\left[n \lambda_{k}\right]+1}\right)^{\prime}, \\
1_{k} & =(1, \cdots, 1)^{\prime} \text { a } k \text { tuple of } 1 \text { 's } \\
\text { and } Q_{0}(\lambda) & =\left(Q_{0}\left(\lambda_{1}\right), \cdots, Q_{0}\left(\lambda_{k}\right)\right)^{\prime} .
\end{aligned}
$$

Thus, ABLUE of $\left(\beta_{0}, \sigma\right)^{\prime}$ based on $Y_{(\cdot)}$ is given by

$$
\begin{gathered}
\hat{\beta}_{0}=\frac{1}{\Delta}\left\{K_{2} M_{1}-K_{3} M_{2}\right\}=a_{1} Y_{\left[n \lambda_{1}\right]+1}+\cdots+a_{k} Y_{\left[n \lambda_{k}\right]+1}, \\
\hat{\sigma}=\frac{1}{\Delta}\left\{K_{1} M_{2}-K_{3} M_{1}\right\}=b_{1} Y_{\left[n \lambda_{1}\right]+1}+\cdots+b_{k} Y_{\left[n \lambda_{k}\right]+1}
\end{gathered}
$$

and

$$
\begin{aligned}
a_{i}= & \frac{q_{0}\left(\lambda_{i}\right) K_{2}}{\Delta}\left\{\frac{q_{0}\left(\lambda_{i}\right)-q_{0}\left(\lambda_{i-1}\right)}{\lambda_{i}-\lambda_{i-1}}-\frac{q_{0}\left(\lambda_{i+1}\right)-q_{0}\left(\lambda_{i}\right)}{\lambda_{i+1}-\lambda_{i}}\right\}- \\
& \frac{q_{0}\left(\lambda_{i}\right) K_{3}}{\Delta}\left\{\frac{q_{0}\left(\lambda_{i}\right) Q_{0}\left(\lambda_{i}\right)-q_{0}\left(\lambda_{i-1}\right) Q_{0}\left(\lambda_{i-1}\right)}{\lambda_{i}-\lambda_{i-1}}-\frac{q_{0}\left(\lambda_{i+1}\right) Q_{0}\left(\lambda_{i+1}\right)-q_{0}\left(\lambda_{i}\right) Q_{0}\left(\lambda_{i}\right)}{\lambda_{i+1}-\lambda_{i}}\right\}, \\
b_{i}= & \frac{q_{0}\left(\lambda_{i}\right) K_{1}}{\Delta}\left\{\frac{q_{0}\left(\lambda_{i}\right) Q_{0}\left(\lambda_{i}\right)-q_{0}\left(\lambda_{i-1}\right) Q_{0}\left(\lambda_{i-1}\right)}{\lambda_{i}-\lambda_{i-1}}-\frac{q_{0}\left(\lambda_{i+1}\right) Q_{0}\left(\lambda_{i+1}\right)-q_{0}\left(\lambda_{i}\right) Q_{0}\left(\lambda_{i}\right)}{\lambda_{i+1}-\lambda_{i}}\right\} \\
& -\frac{q_{0}\left(\lambda_{i}\right) K_{3}}{\Delta}\left\{\frac{q_{0}\left(\lambda_{i}\right)-q_{0}\left(\lambda_{i-1}\right)}{\lambda_{i}-\lambda_{i-1}}-\frac{q_{0}\left(\lambda_{i+1}\right)-q_{0}\left(\lambda_{i}\right)}{\lambda_{i+1}-\lambda_{i}}\right\}
\end{aligned}
$$


$i=1,2, \cdots, k$, where

$$
\begin{gathered}
K_{1}=1_{k}^{\prime} \Omega^{-1} 1_{k}, \quad K_{2}=Q_{0}(\lambda)^{\prime} \Omega^{-1} Q_{0}(\lambda), \quad \Delta=K_{1} K_{2}-K_{3}^{\prime 2} \\
K_{3}=Q_{0}(\lambda)^{\prime} \Omega^{-1} 1_{k}, \quad M_{1}=1_{k}^{\prime} \Omega^{-1} Y_{(\cdot)} \text { and } M_{2}=Q_{0}(\lambda)^{\prime} \Omega^{-1} Y_{(\cdot)}
\end{gathered}
$$

The variance-covariance matrix of the ABLUE is given by

$$
\frac{\sigma^{2}}{n \Delta}\left(\begin{array}{cc}
K_{2} & -K_{3} \\
-K_{3} & K_{1}
\end{array}\right) \text {. }
$$

The explicit expressions for $K_{1}, K_{2}$ and $K_{3}$ are given by

$$
\begin{aligned}
& K_{1}=\sum_{i=1}^{k} \frac{\left[q_{0}\left(\lambda_{i}\right)-q_{0}\left(\lambda_{i-1}\right)\right]^{2}}{\lambda_{i}-\lambda_{i-1}} \\
& K_{2}=\sum_{i=1}^{k} \frac{\left[q_{0}\left(\lambda_{i}\right) Q_{0}\left(\lambda_{i}\right)-q_{0}\left(\lambda_{i-1}\right) Q_{0}\left(\lambda_{i-1}\right)\right]^{2}}{\lambda_{i}-\lambda_{i-1}} \\
& K_{3}=\sum_{i=1}^{k} \frac{\left[q_{0}\left(\lambda_{i}\right)-q_{0}\left(\lambda_{i-1}\right)\right]\left[q_{0}\left(\lambda_{i}\right) Q_{0}\left(\lambda_{i}\right)-q_{0}\left(\lambda_{i-1}\right) Q_{0}\left(\lambda_{i-1}\right)\right]}{\lambda_{i}-\lambda_{i-1}} .
\end{aligned}
$$

The joint asymptotic relative efficiency (JARE) of the ABLUE of $\beta_{0}$ and $\sigma$ may be obtained by the expression for the regular cases as

$$
J A R E[A B L U E: M L E]=\frac{K_{1} K_{2}-K_{3}^{2}}{I_{11} I_{22}-I_{12}^{2}},
$$

where

$$
I=\left(\begin{array}{ll}
I_{11} & I_{12} \\
I_{12} & I_{22}
\end{array}\right)
$$

is the information matrix for the location-scale distribution. The ARE of $\hat{\beta}_{0}$ or $\hat{\sigma}$ are then given by

$$
A R E\left[\hat{\beta}_{0}: M L E \text { of } \beta_{0}\right]=\frac{\left(K_{1} K_{2}-K_{3}^{2}\right) I_{22}}{K_{2}\left(I_{11} I_{22}-I_{12}^{2}\right)}
$$

and

$$
A R E[\hat{\sigma}: \text { MLE of } \sigma]=\frac{\left(K_{1} K_{2}-K_{3}^{2}\right) I_{11}}{K_{1}\left(I_{11} I_{22}-I_{12}^{2}\right)}
$$


Note that $I_{12}=0$ for symmetric distributions. Elements of information matrix are considered below for some symmetric distributions.

\begin{tabular}{|c|c|c|c|} 
Distribution & $I_{11}$ & $I_{22}$ & $I_{12}$ \\
\hline Normal & 1 & 2 & 0 \\
Cauchy & $1 / 2$ & $1 / 2$ & 0 \\
Student's $(\nu$ d.f) & $\frac{v+1}{u+3}$ & $\frac{2 \nu}{v+3}$ & 0 \\
Logistic & $\frac{\frac{2}{2}^{2}}{0}$ & $\frac{3+\pi^{2}}{0}$ & 0 \\
Doublc-Exp & 1 & 1 & 0 \\
\hline
\end{tabular}

Naturally, the question of optimum spacing vector arises and may be obtained by maximizing (2.41), (2.43) and (2.44) by choosing $Q_{0}\left(\lambda_{1}\right), \cdots, Q_{0}\left(\lambda_{k}\right)$ appropriately. Some numerical results on optimum spacings are given in appendix $A$ for the $t$ distribution with $\nu$ degrees of freedom for $\nu=1(1) 3,5,10,20,500$ and $k=2(1) 10$.

In this section, we have discussed the so-called inefficient statistics due to Mosteller's (1946) initiatives based on a few sample quantiles as quick and dirfy substitutes for more conventional estimators. It can be seen that the estimators of this type are almost as efficient as the maximum likelihood estimators. Gastwirth (1966) and others established some estimators of this type with good efficiency properties for a variety of distribution: Our check with the class of t-distributions provides such evidence of efficiency as well. Although these quick and dirty estimators are slightly inefficient in a fixed parametric model, in practice they may actually be preferable to putatively optimal estimators like the sample mean, if there is some uncertainty about the shape of the underlying distribution. These simple estimators may be called simplified L-estimators. Recent work has devoted to extending results of this type beyond a fixed number of quantiles. The asymptotic theory of such L-estimators has achieved classical standing through the efforts of Chernoff et al (1967) and Bickel (1968). The most common L-estimator of location is the $\lambda$-trimmed mean which is simply the mean of the sample after the proportion $\lambda$ of largest and smallest observations have been removed. In the next section we discuss this estimator with the 
asymptotic theory.

\subsection{Trimmed Least-Squared Estimation of Loca- tion Parameter And Its Asymptotic Distri- bution}

We have considered the Lestimation of location and scale parameters based on all order statistics or on a few optimum sample quantiles which resulted in quick estimator of $\beta_{0}$ and $\sigma$ for each distribution under consideration, sacrificing very little on the asymptotic relative efficiency. The idea of derivation was based on the generalized least squares principle.

For the location model

$$
Y=\beta_{0} 1_{n}+Z,
$$

where $Z=\left(Z_{1}, \cdots, Z_{n}\right)^{\prime}$ have components which are i.i.d r.v's from a continuous cdf $F_{0}(z)$ (with $p d f f_{0}(z)$ ), the least squares estimator (LSE) of $\beta_{0}$ is the sample mean $\overline{\boldsymbol{Y}}_{n}$ given by

$$
\bar{Y}_{n}=\left(1_{n}^{\prime} 1_{n}\right)^{-1} 1_{n}^{\prime} Y
$$

Tukey (1960) proposed the trimmed estimator of $\beta_{0}$ as

$$
\bar{Y}_{n}(r)=\frac{1}{n-2 r}\left\{Y_{(r+1)}+\cdots+Y_{(n-r)}\right\},
$$

where $r$ is the number of trimmed order statistics from the bottom and the top. This estimator was the integral part of the Huber's (1981) robust estimator of $\beta_{0}$. We obtain a similar estimator by first defining the $n \times n$ diagonal matrix $A$ whose elements are

$$
a_{i i}=\left\{\begin{array}{lc}
1, & \text { if } \bar{Q}_{n}\left(\lambda_{1}\right)<Y_{i} \leq \bar{Q}_{n}\left(\lambda_{2}\right) \\
0, & \text { otherwise, }
\end{array}\right.
$$


where $Q_{n}\left(\lambda_{1}\right)$ and $Q_{n}\left(\lambda_{2}\right)$ are the sample quantiles of order $\lambda_{1}$ and $\lambda_{2}$ respectively, $\left(0<\lambda_{1}<1 / 2<\lambda_{2}<1\right)$ used for trimming the sample. Thus, minimizing

$$
\left(\boldsymbol{Y}-\boldsymbol{\beta}_{0} 1\right)^{\prime} A\left(\boldsymbol{Y}-\boldsymbol{\beta}_{0} 1\right)
$$

w.r.t $\beta_{0}$, we obtain the trimmed least squares estimator (TLSE) as follows:

$$
\bar{\beta}_{0 n}\left(\lambda_{1}, \lambda_{2}\right)=\left(1_{n}^{\prime} A 1_{n}\right)^{-1}\left(1_{n}^{\prime} A Y\right)
$$

The objective of this section is to provide an asymptotic linearity (AL) result for this statistic. Before we proceed with the representation of $\bar{\beta}_{0 n}\left(\lambda_{1}, \lambda_{2}\right)$, we present some of the historical results regarding sample quantiles. The sample quantiles are themselves excellent summary data and fascinating random variables so much so that many researchers have devoted their life-time research to their study. One such result is due to Bahadur (1966) giving a representation of sample quantiles, namely,

$$
\bar{Q}_{n}(\lambda)-\beta_{0}-Q_{0}(\lambda)=n^{-1} \sum_{i=1}^{n} \Psi_{F}\left(Z_{1}\right)+O\left(n^{-3 / 4} \log n\right) \quad \text { a.s. }
$$

where

$$
\Psi_{F}\left(Z_{i}\right)=-\left[q_{0}(\lambda)\right]^{-1}\left\{I\left(Z_{i} \leq Q_{0}(\lambda)\right)-\lambda\right\}
$$

and when $F_{0}$ has at least two derivatives in the neighborhood of $Q_{0}(\lambda)$ and is bounded in that neighborhood and $q_{0}(\lambda)>0$. Note that the R.H.S of $(2.51)$ is the sum of i.i.d. r.v. Hence, the asymptotic joint distribution of severa! sample quantiles follow easily than using the density method due to Mosteller (1946). More apt is the strong approximation results due to Csörgö and Révész (1975). First, we recall the definition of Brownian bridge process $\{B(\lambda): 0<\lambda<1\}$; it is a zero-mean normal process with covariance function

$$
K_{B}\left(\lambda_{1}, \lambda_{2}\right)=\lambda_{1} \wedge \lambda_{2}-\lambda_{1} \lambda_{2}
$$

Theorem 2.4.1 (Csörgö and Révész, 1975) A Brownian bridge $\left\{B_{n}(\lambda): 0<\right.$ $\lambda<1\}$ can be defined for each $n$ such that almost surely

$$
\sup _{0<\lambda<1}\left|n^{1 / 2}\left\{\bar{Q}_{n}(\lambda)-\lambda\right\}-B_{n}(\lambda)\right|=O\left(n^{-1 / 2} \log n\right)
$$


where $Q_{n}(\lambda)$ is the quantile of order $\lambda$ from a sample of size $n$ from the uniform distribution.

For the purpuse of statistical inference, we can interpret the forgoing result as follows for the location-scale case

$$
\frac{n^{1 / 2}}{\sigma} q_{0}(\lambda)\left\{Q_{n}(\lambda)-\beta_{0}-\sigma Q_{0}(\lambda)\right\} \sim B(\lambda)
$$

For izstance, see Parzen (1979). Eubank (1981) in his Ph.D thesis developed the results of Ogawa (1951) for the estimation of $\beta_{0}$ and $\sigma$ based on a few selected sample quantiles using the result in (2.55). Further, he defined optimum density function which generates the optimum spacings for the ABLUE of $\beta_{0}$ and $\sigma$.

Now, we return to the TLSE case. In this case, we need only a weaker representation for $\bar{Q}_{n}(\lambda)$ as given by

$$
\bar{Q}_{n}(\lambda)-\beta_{0}-Q_{0}(\lambda)=-n^{-1}\left[g_{0}(\lambda)\right]^{-1} \sum_{i=1}^{n}\left\{I\left(Z_{i} \leq Q_{0}(\lambda)\right)-\lambda\right\}+o_{P}(1) .
$$

We are going to show this representation under similar condition as we need to represent $\bar{\beta}_{0 n}\left(\lambda_{1}, \lambda_{2}\right)$ given at $(2.50)$. First, we consider the model $(2.45)$ and the process

$$
T_{n}(t ; \lambda)=n^{-1 / 2} \sum_{i=1}^{n}\left\{I\left(Y_{i}-t \leq 0\right)-\lambda\right\}
$$

Now, defining

$$
\beta_{0}(\lambda)=\beta_{0}+Q_{0}(\lambda)
$$

we have the process $(2.57)$ at $\beta_{0}(\lambda)$ given by

$$
T_{n}\left(\beta_{0}(\lambda) ; \lambda\right)=n^{-1 / 2} \sum_{i=1}^{n}\left\{I\left(Z_{i} \leq Q_{0}(\lambda)\right)-\lambda\right\}
$$

As a result, we have the following theorem under the condition F1:

F1 $F_{0}$ is continuous and has positive and continuous density function $f_{0}$ in the neighborhood of $Q_{0}(\lambda)$. 
Theorem 2.4.2 Under the condition F1

$$
n^{1 / 2}\left(\bar{Q}_{n}(\lambda)-\beta_{0}(\lambda)\right)=-\left[q_{0}(\lambda)\right]^{-1} T_{n}\left(\beta_{0}(\lambda) ; \lambda\right)+o_{P}(1)
$$

The theorem is proved based on the following two lemmas with new proofs.

Lemma 2.4.3 Let the condition F1 hold. Then, for each $b>0$

$$
\sup _{|1| \leq b}\left|T_{r}\left(\beta_{0}(\lambda)+n^{-1 / 2} t ; \lambda\right)-T_{n}\left(\beta_{0}(\lambda) ; \lambda\right)-t q_{0}(\lambda)\right|=o_{P}(1)
$$

\section{Proof:}

The expression (2.61) is bounded above by

$$
\begin{aligned}
\sup _{|1| \leq b} \mid & \left\{T_{n}\left(\beta_{0}(\lambda)+n^{-1 / 2} t ; \lambda\right)-E\left[T_{n}\left(\beta_{0}(\lambda)+n^{-1 / 2} t ; \lambda\right)\right]\right\} \\
- & \left\{T_{n}\left(\beta_{0}(\lambda) ; \lambda\right)-E\left[T_{n}\left(\beta_{0}(\lambda) ; \lambda\right)\right]\right\} \mid \\
+\sup _{|1| \leq b} \mid & E\left[T_{n}\left(\beta_{0}(\lambda)+n^{-1 / 2} t ; \lambda\right)\right]-E\left[T_{n}\left(\beta_{0}(\lambda) ; \lambda\right)\right]-t q_{0}(\lambda) \mid \\
= & I_{1}+I_{2} .
\end{aligned}
$$

Rewriting $I_{1}$ and $I_{2}$ as

$$
I_{1}=\sup _{|t| \leq b}\left|T_{n}^{0}(t ; \lambda)-T_{n}^{0}(0 ; \lambda)\right|
$$

where

$$
T_{n}^{0}(t ; \lambda)=T_{n}\left(\beta_{0}(\lambda)+n^{-1 / 2} t ; \lambda\right)-E\left[T_{n}\left(\beta_{0}(\lambda)+n^{-1 / 2} t ; \lambda\right)\right]
$$

and

$$
\left.I_{2}=\sup _{|\lambda| \leq b} n^{1 / 2} \mid F_{0}\left(Q_{0}(\lambda)+n^{-1 / 2} t\right)-F_{0}\left(Q_{0}(\lambda)\right)-n^{-1 / 2} t q_{0}(\lambda)\right) \mid
$$

First, we consider $I_{2}$. Under condition F1 and using the mean-value theorem, we get

$$
I_{2} \leq b \cdot \sup _{|\Delta(t)| \leq \delta_{n}}\left|f_{0}\left(Q_{0}(\lambda)+\Delta(t)\right)-f_{0}\left(Q_{0}(\lambda)\right)\right|
$$


where $\delta_{n}=n^{-1 / 2}$ b. So, $\forall \epsilon>0 \exists N^{e}$ and $\delta>0$ such that $\delta_{n}<\delta \forall n>N^{c}$. Therefore, by $\mathrm{Fl}$ we have $I_{2} \leq \epsilon$.

Now, we consider the following set

$$
\mathcal{N}(b)=\{t \in \mathbb{R}:|t| \leq b\}
$$

For $I_{1}$, first we will show that $I_{1}=\operatorname{op}(1)$ for each $t \in \mathcal{N}(b)$ where

$$
\begin{aligned}
I_{1}= & \left|T_{n}^{0}(t ; \lambda)-T_{n}^{0}(0 ; \lambda)\right| \\
= & n^{-1 / 2} \mid \sum_{i=1}^{n} I\left(Z_{i} \leq Q_{0}(\lambda)+n^{-1 / 2} t\right)-F_{0}\left(Q_{0}(\lambda)+n^{-1 / 2} t\right) \\
& \quad-I\left(Z_{i} \leq Q_{0}(\lambda)\right)+F_{0}\left(Q_{0}(\lambda)\right) \mid \\
= & n^{-1 / 2}\left|\sum_{i=1}^{n}\left(V_{i}(t)-\nu_{n}(t)\right)\right|
\end{aligned}
$$

where

$$
\begin{aligned}
& V_{i}(t)=I\left(Q_{0}(\lambda)<Z_{i} \leq Q_{0}(\lambda)+n^{-1 / 2} t\right) \\
& \nu_{n}(t)=E\left(V_{i}(t)\right)=F_{0}\left(Q_{0}(\lambda)+n^{-1 / 2} t\right)-F_{0}\left(Q_{0}(\lambda)\right) .
\end{aligned}
$$

Then, for any $\epsilon>0$

$$
\begin{aligned}
P\left\{\left|n^{-1 / 2} \sum_{i=1}^{n}\left(V_{i}(t)-\nu_{n}(t)\right)\right|^{2}>\epsilon\right\} & \leq \frac{1}{\epsilon^{2}} E\left(n^{-1 / 2} \sum_{i=1}^{n}\left(V_{i}(t)-\nu_{n}(t)\right)\right)^{2} \\
& =\frac{1}{\epsilon^{2}} \frac{1}{n} \sum_{i=1}^{n} E\left(V_{i}(t)-\nu_{n}(t)\right)^{2} \\
& =\frac{1}{\epsilon^{2}}\left(\nu_{n}(t)-\nu_{n}^{2}(t)\right) .
\end{aligned}
$$

But, $\nu_{n}(t) \rightarrow 0$ as $n \rightarrow \infty$ by continuity of $F_{0}$. Therefore, $I_{1}=o_{P}(1)$. For the tightness of the process we use the compactness property of the set $\mathcal{N}(b)$. Since the set $\mathcal{N}(b)$ is compact, it suffices to show that for every $\epsilon>0$, there is a $\delta>0$ such that for each $s \in \mathcal{N}(b)$,

$$
\limsup _{n} P\left(\sup _{|t-s| \leq \delta}\left|T_{n}^{0}(t ; \lambda)-T_{n}^{0}(s ; \lambda)\right|>2 \epsilon\right)<\epsilon .
$$


Using the definition of $T_{n}^{0}(\cdot ; \cdot)$ we have

$$
\begin{aligned}
T_{n}^{0}(t ; \lambda)-T_{n}^{0}(s ; \lambda) & n^{-1 / 2} \sum_{i=1}^{n}\left\{\left(I\left(Z_{i} \leq Q_{0}(\lambda)+n^{-1 / 2} t\right)-I\left(Z_{i} \leq Q_{0}(\lambda)+n^{-1 / 2} s\right)\right)\right. \\
& \left.-\left(F_{0}\left(Q_{0}(\lambda)+n^{-1 / 2} t\right)-F_{0}\left(Q_{0}(\lambda)+n^{-1 / 2} s\right)\right)\right\} \\
= & n^{-1 / 2} \sum_{i=1}^{n}\left\{\left(\alpha_{i}(t)-\alpha_{i}(s)\right)-\left(\mu_{n}(t)-\mu_{n}(s)\right)\right\}
\end{aligned}
$$

where

$$
\alpha_{i}(t)=I\left(Z_{i} \leq Q_{0}(\lambda)+n^{-1 / 2} t\right) \text { and } \mu_{n}(t)=E\left(\alpha_{i}(t)\right)
$$

Now, using the monotone property of the indicator function with the relationship between $s$ and $t$, i.e

$$
s-\delta \leq t \leq s+\delta
$$

we have

$$
\begin{aligned}
& T_{n}^{0}(t ; \lambda)-T_{n}^{0}(s ; \lambda) \\
& \leq n^{-1 / 2} \sum_{i=1}^{n}\left\{\alpha_{i}(s+\delta)-\alpha_{i}(s)-\mu_{n}(s-\delta)+\mu_{n}(s)\right\} \\
& =n^{-1 / 2} \sum_{i=1}^{n}\left\{\alpha_{i}(s+\delta)-\alpha_{i}(s)-\mu_{n}(s+\delta)+\mu_{n}(s)\right. \\
& \left.\quad \quad+\mu_{n}(s+\delta)-\mu_{n}(s-\delta)\right\} \\
& \quad=T_{n}^{0}(s+\delta ; \lambda)-T_{n}^{0}(s ; \lambda)+n^{1 / 2}\left\{\mu_{n}(s+\delta)-\mu_{n}(s-\delta)\right\}
\end{aligned}
$$

Similarly, we have

$$
\begin{aligned}
T_{n}^{0}(s-\delta ; \lambda)-T_{n}^{0}(s ; \lambda)+n^{1 / 2}\left\{\mu_{n}(s-\delta)-\mu_{n}(s+\delta)\right\} \\
\leq T_{n}^{00}(t ; \lambda)-T_{n}^{0}(s ; \lambda) \leq T_{n}^{0}(s+\delta ; \lambda)-T_{n}^{0}(s ; \lambda) \\
\quad+n^{1 / 2}\left\{\mu_{n}(s+\delta)-\mu_{n}(s-\delta)\right\}
\end{aligned}
$$


Thus,

$$
\begin{aligned}
\left|T_{n}^{0}(t ; \lambda)-T_{n}^{0}(s ; \lambda)\right| \leq\left|T_{n}^{0}(s+\delta ; \lambda)-T_{n}^{0}(s ; \lambda)\right| & \\
& +\left|T_{n}^{0}(s-\delta ; \lambda)-T_{n}^{0}(s ; \lambda)\right| \\
& +2 n^{1 / 2}\left|\mu_{n}(s+\delta)-\mu_{n}(s-\delta)\right| \\
& =I_{11}+I_{12}+I_{13}=o_{P}(1) \text { as } n \rightarrow \infty
\end{aligned}
$$

By continuity of $F_{0}$ we have $\left|\mu_{n}(s+\delta)-\mu_{n}(s)\right| \rightarrow 0$ as $n \rightarrow \infty$ and $I_{11}=o_{P}(1)=I_{12}$ by previous discussion. Hence the lemma.

The next step is to show that the sample quantiles are bounded in probability, which we state below:

Lemma 2.4.4 Under the condition $F 1$, for each $\lambda \in(0,1)$,

$$
n^{1 / 2}\left(\bar{Q}_{n}(\lambda)-\beta_{0}(\lambda)\right)=O_{P}(1)
$$

\section{Proof:}

This Lemma is proved by two different methods. For the first one we assume that $f_{0}\left(F_{0}^{-1}(\lambda)\right)>0$ and $t$ be any positive real number. If we take $k_{n}=n \lambda+o\left(n^{-1 / 2}\right)$, we have

$$
\begin{aligned}
P & \left\{Y_{\left(k_{n}\right)} \leq \beta_{0}(\lambda)-n^{-1 / 2} t\right\} \\
= & P\left\{F_{n}\left(\beta_{0}(\lambda)-n^{-1 / 2} t\right) \geq n^{-1} k_{n}\right\} \\
= & P\left\{n^{1 / 2}\left[F_{n}\left(\beta_{0}(\lambda)-n^{-1 / 2} t\right)-F\left(\beta_{0}(\lambda)-n^{-1 / 2} t\right)\right]\right. \\
& \left.\geq n^{1 / 2}\left[n^{-1} k_{n}-F\left(\beta_{0}(\lambda)-n^{-1 / 2} t\right)\right]\right\}
\end{aligned}
$$

$\leq \epsilon$.

Since

$$
n^{1 / 2}\left[n^{-1} k_{n}-F\left(\beta_{0}(\lambda)-n^{-1 / 2} t\right)\right]=t q_{0}(\lambda)+o(1)
$$


by Taylor's expansion while $n^{1 / 2}\left[F_{n}(x)-F(x)\right]$ is asymptotically normal with mean 0 and finite variance, by taking $t$ large enough we get the result for sufficiently large n. A similar result holds for the right tail as well and the proof o the Lemma is complete.

The second method is based on Jureckova (1984) and is the one which we shall follow in subsequent chapters involving regression and autoregression models. We need the following steps to prove this Lemma

$$
\text { i) } \quad T_{n}\left(\bar{Q}_{n}(\lambda) ; \lambda\right)=o_{P}(1)
$$

ii) $\forall c>0,0<u<\propto \exists b\left(=b_{u}^{e}\right)$ and an $N^{e}$ such that

$$
\text { for each } \lambda \in(0,1)
$$

$$
P\left(\inf _{|l|>b}\left|T_{n}\left(\beta_{0}(\lambda)+n^{-1 / 2} t ; \lambda\right)\right|>u\right)>1-\epsilon
$$

Proof of (i)

Follows from Lemma A.2 of Ruppert and Carroll (1980). Lemma A.2 of Ruppert and Carroll (1980) in this case is:

The sequence of solutions $\bar{Q}_{n}(\lambda)$ of (2.22) satisfies

$$
n^{-1 / 2} \sum_{i=1}^{n}\left\{I\left(y_{i}-\bar{Q}_{n}(\lambda) \leq 0\right)-\lambda\right\} \rightarrow 0 \quad \text { a.s. }
$$

ProOF of (ii)

To prove this, we shall follow the Koul and Saleh (1995) approach. By using the non-decreasing property of $T_{n}\left(\beta_{0}(\lambda)+n^{-1 / 2} t ; \lambda\right)$ with respect to $t$, we have

$$
\inf _{|t|>b}\left|T_{n}\left(\beta_{0}(\lambda)+n^{-1 / 2} t ; \lambda\right)\right|^{2} \geq \inf _{|t|=b}\left|T_{n}\left(\beta_{0}(\lambda)+n^{-1 / 2} t ; \lambda\right)\right|^{2}
$$

for each $\lambda \in(0,1)$. Let us define

$$
T_{n}^{*}(t ; \lambda)=T_{n}\left(\beta_{0}(\lambda) ; \lambda\right)+t q_{0}(\lambda)
$$

By Lemma 2.4.3 we have

$$
T_{n}\left(\beta_{0}(\lambda)+n^{-1 / 2} t ; \lambda\right)-T_{n}^{*}(t ; \lambda)=o_{P}(1) .
$$


Then, for all $\epsilon>0$ there is an $N^{2}$ such that

$$
\begin{aligned}
& P\left(\inf _{|t|=b}\left\{T_{n}\left(\beta_{0}(\lambda)+n^{-1 / 2} t ; \lambda\right)\right\}^{2}>u\right) \\
& \quad \geq P\left(\inf _{|| \mid=b}\left\{T_{n}^{*}(t ; \lambda)\right\}^{2}>u\right)-\epsilon / 3, \quad n>N^{e}
\end{aligned}
$$

Thus, it is enough to see the behavior of $T_{n}^{*}(t ; \lambda)$. Now, using the absolute value property, we have

$$
\begin{aligned}
& P\left(\inf _{|k|=b}\left\{T_{n}^{*}(t ; \lambda)\right\}^{2}>u\right) \\
& \geq P\left(|| T_{n}\left(\beta_{0}(\lambda) ; \lambda\right)\left|-b q_{0}(\lambda)\right|>u^{1 / 2}\right) \\
& \geq P\left(\left|T_{n}\left(\beta_{0}(\lambda) ; \lambda\right)\right| \leq-u^{1 / 2}+b q_{0}(\lambda)\right) \\
& \geq 1-(2 \epsilon / 3), \quad \forall n>N^{e}
\end{aligned}
$$

where $b>\frac{x^{1 / 2}+M}{90(\lambda)}$. Equation (2.88) results from the fact that $T_{n}\left(\beta_{0}(\lambda) ; \lambda\right)$ is a sum of $i . i . d$ random variables which by C.L.T converges to a normal distribution as $n \rightarrow \infty$. Let $\mathrm{Z}$ be a standard normal variable. Then, $\forall \epsilon>0 \exists M_{c}>0$ and $N^{c} \ni$

$$
P\left(\left|T_{n}\left(\beta_{0}(\lambda) ; \lambda\right)\right| \leq M_{c}\right)>1-(2 \epsilon / 3) \quad \forall n>N^{c}
$$

Now, we apply the results of (2.81) and (2.82) to complete the proof of Lemma 2.4.4. We observe that for any $b>0$

$$
\begin{aligned}
P\left(n^{1 / 2}\left|\bar{Q}_{n}(\lambda)-\beta_{0}(\lambda)\right|>b\right) & \\
= & P\left(n^{1 / 2}\left|\bar{Q}_{n}(\lambda)-\beta_{0}(\lambda)\right|>b,\left|T_{n}\left(\beta_{0}(\lambda)+n^{-1 / 2}\left[n^{1 / 2}\left(\bar{Q}_{n}(\lambda)-\beta_{0}(\lambda)\right)\right] ; \lambda\right)\right|<u\right) \\
& +P\left(n^{1 / 2}\left|\bar{Q}_{n}(\lambda)-\beta_{0}(\lambda)\right|>b,\left|T_{n}\left(\beta_{0}(\lambda)+n^{-1 / 2}\left[n^{1 / 2}\left(\bar{Q}_{n}(\lambda)-\beta_{0}(\lambda)\right)\right] ; \lambda\right)\right| \geq u\right) \\
\leq & P\left(\inf _{|z|>b}\left|T_{n}\left(\beta_{0}(\lambda)+n^{-1 / 2} t ; \lambda\right)\right|<u\right)+P\left(\left|T_{n}\left(\bar{Q}_{n}(\lambda) ; \lambda\right)\right| \geq u\right) \\
\leq & \epsilon / 2+c / 2 \quad \text { by }(2.81) \text { and }(2.82) \quad \forall n>N^{\epsilon} .
\end{aligned}
$$


Now by using Lemmas 2.4.3 and 2.4.4 we get the representation of $Q_{n}(\lambda)$. By Lemma 2.4 .3 for every bounded $t$,

$$
T_{n}\left(\beta_{0}(\lambda)+n^{-1 / 2} t ; \lambda\right)-T_{n}\left(\beta_{0}(\lambda) ; \lambda\right)-t g_{0}(\lambda)=o_{P}(1)
$$

Hence, by applying the Lemma 2.4.4 with $t=n^{1 / 2}\left(Q_{n}(\lambda)-\beta_{0}(\lambda)\right)$, we have

$$
\begin{aligned}
& T_{n}\left(\beta_{0}(\lambda)+n^{-1 / 2}\left[n^{\frac{1}{2}}\left(Q_{n}(\lambda)-\beta_{0}(\lambda)\right)\right] ; \lambda\right)-T_{n}\left(\beta_{0}(\lambda) ; \lambda\right)-q_{0}(\lambda) n^{\frac{1}{1}}\left(Q_{n}(\lambda)-\beta_{0}(\lambda)\right) \\
& =o_{P}(1) .
\end{aligned}
$$

On the other hand

$$
T_{n}\left(\bar{Q}_{n}(\lambda) ; \lambda\right)-T_{n}\left(\beta_{0}(\lambda) ; \lambda\right)-\varphi_{0}(\lambda) n^{\frac{1}{2}}\left(\bar{Q}_{n}(\lambda)-\beta_{0}(\lambda)\right)=o_{P}(1),
$$

and (2.81) implies

$$
\begin{aligned}
n^{1 / 2}\left(\bar{Q}_{n}(\lambda)-\beta_{0}(\lambda)\right) & =-\left[q_{0}(\lambda)\right]^{-1} T_{n}\left(\beta_{0}(\lambda) ; \lambda\right)+o_{P}(1) \\
& =-n^{-1 / 2}\left[q_{0}(\lambda)\right]^{-1} \sum_{i=1}^{n}\left\{I\left(Z_{i} \leq Q_{0}(\lambda)\right)-\lambda\right\}+o_{P}(1)
\end{aligned}
$$

As we mentioned before, the object of this section is to represent the TLSE of $\beta_{0}$ given in (2.50). Thus, we have

Theorem 2.4.5 Let $F_{0}$ be continuous with continuous and positive density function $f_{0}$ in the neighborhood of $Q_{0}\left(\lambda_{1}\right)$ and $Q_{0}\left(\lambda_{2}\right)$ respectively. Then

$$
\begin{gathered}
\text { i) } \quad n^{1 / 2}\left(\bar{\beta}_{0 n}\left(\lambda_{1}, \lambda_{2}\right)-\beta_{0}\right) \\
=\left(\lambda_{2}-\lambda_{1}\right)^{-1} n^{-1 / 2} \sum_{i=1}^{n}\left(\phi\left(Z_{i}\right)-\gamma\right)+o_{P}(1) \\
\text { ii) } \quad n^{1 / 2}\left(\bar{\beta}_{0 n}\left(\lambda_{1}, \lambda_{2}\right)-\beta_{0}\right) \stackrel{D}{\rightarrow} \mathcal{N}\left(0, \sigma^{2}\left(\lambda_{1}, \lambda_{2}\right)\right),
\end{gathered}
$$

where

$$
\begin{aligned}
& \phi(Z)=\left\{\begin{array}{ccc}
Q_{0}\left(\lambda_{1}\right), & \text { if } & Z<Q_{0}\left(\lambda_{1}\right), \\
Z, & \text { if } & Q_{0}\left(\lambda_{1}\right)<Z \leq Q_{0}\left(\lambda_{2}\right), \\
Q_{0}\left(\lambda_{2}\right), & \text { if } & Z>Q_{0}\left(\lambda_{2}\right)
\end{array}\right. \\
& \gamma=\lambda_{1} Q_{0}\left(\lambda_{1}\right)+\left(1-\lambda_{2}\right) Q_{0}\left(\lambda_{2}\right)
\end{aligned}
$$


CHAPTER 2. LOCATION AND SCALE MODEL

26

and

$$
\begin{aligned}
& \sigma^{2}\left(\lambda_{1}, \lambda_{2}\right) \\
& =\left(\lambda_{2}-\lambda_{1}\right)^{-2}\left\{\int_{\lambda_{1}}^{\lambda_{2}}\left(Q_{0}(u)-\delta_{0}\right)^{2} d u+\lambda_{1}\left(Q_{0}\left(\lambda_{1}\right)-\delta_{0}\right)^{2}\right. \\
& \left.\quad+\left(1-\lambda_{2}\right)\left(Q_{0}\left(\lambda_{2}\right)-\delta_{0}\right)^{2}-\left[\lambda_{1}\left(Q_{0}\left(\lambda_{1}\right)-\delta_{0}\right)+\left(1-\lambda_{2}\right)\left(Q_{0}\left(\lambda_{2}\right)-\delta_{0}\right)\right]^{2}\right\}
\end{aligned}
$$

with

$$
\delta_{0}=\left(\lambda_{2}-\lambda_{1}\right)^{-1} \int_{\lambda_{1}}^{\lambda_{2}} Q_{0}(u) d u
$$

Proof: We shall prove this Theorem based on the two Lemmas (2.4.6 and 2.4.7). First, we define the following processes

$$
\tilde{T}_{n}(t ; \lambda)=n^{-1 / 2} \sum_{i=1}^{n} Z_{i} I\left(Z_{i} \leq Q_{0}(\lambda)+n^{-1 / 2} t\right)
$$

and

$$
\begin{aligned}
\dot{T}_{n}^{0}(t ; \lambda) & =\dot{T}_{n}(t ; \lambda)-E\left(\dot{T}_{n}(t ; \lambda)\right) \\
& =n^{-1 / 2} \sum_{i=1}^{n}\left\{Z_{i} I\left(Z_{i} \leq Q_{0}(\lambda)+n^{-1 / 2} t\right)-\mu\left(Q_{0}(\lambda)+n^{-1 / 2} t\right)\right\}
\end{aligned}
$$

where

$$
\mu\left(Q_{0}(\lambda)+n^{-1 / 2} t\right)=E\left[Z_{i} I\left(Z_{i} \leq Q_{0}(\lambda)+n^{-1 / 2} t\right)\right]
$$

and

$$
\mu^{\prime}(u)=u f(u)
$$

Then, we have the Lemma 2.4.6.

Lemma 2.4.6 Under the condition F1 for each $b>0$,

$$
\sup _{|l| \leq b}\left|\tilde{T}_{n}(t ; \lambda)-\tilde{T}_{n}(0 ; \lambda)-t q_{0}(\lambda) Q_{0}(\lambda)\right|=o_{P}(1)
$$




\section{Proof:}

$$
\begin{aligned}
& \sup _{|t| \leq b}\left|\tilde{T}_{n}(t ; \lambda)-\tilde{T}_{n}(0 ; \lambda)-t q_{0}(\lambda) Q_{0}(\lambda)\right| \\
& \quad \leq \sup _{\| 1 \leq b}\left|\tilde{T}_{n}^{0}(t ; \lambda)-\tilde{T}_{n}^{0}(0 ; \lambda)\right|+\sup _{\| t \leq b}\left|E\left(\tilde{T}_{n}(t ; \lambda)\right)-E\left(\tilde{T}_{n}(0 ; \lambda)\right)-t q_{0}(\lambda) Q_{0}(\lambda)\right| \\
& =J_{1}+J_{2} .
\end{aligned}
$$

First, note that

$$
J_{2}=\sup _{|t| \leq b} n^{1 / 2}\left|\mu\left(Q_{0}(\lambda)+n^{-1 / 2} t\right)-\mu\left(Q_{0}(\lambda)\right)-n^{-1 / 2} Q_{0}(\lambda) q_{0}(\lambda) t\right| .
$$

Then by using the mean value theorem, we have

$$
J_{2} \leq b \cdot Q_{0}(\lambda) \sup _{|\Delta(t)| \leq \delta_{n}}\left|f_{0}\left(Q_{0}(\lambda)+\Delta(t)\right)-f_{0}\left(Q_{0}(\lambda)\right)\right|+c_{n} .
$$

Hence R.H.S of (2.107) is o(1) as $n \rightarrow \infty$ by condition F1. Now, to prove the Lemma 2.4.6, it is enough to show that $J_{1}=O_{P}(1)$ where

$$
J_{1}=\sup _{|\ell| \leq b}\left|\tilde{T}_{n}^{0}(t ; \lambda)-\tilde{T}_{n}^{0}(0 ; \lambda)\right| \text {. }
$$

Here, it is necessary to show that for each $t \in \mathcal{N}(b)$

$$
\left|\tilde{T}_{n}^{0}(t ; \lambda)-\tilde{T}_{n}^{0}(0 ; \lambda)\right|=o_{P}(1)
$$

where

$$
\begin{aligned}
& \left|\dot{T}_{n}^{0}(t ; \lambda)-\dot{T}_{n}^{0}(0 ; \lambda)\right| \\
& =n^{-1 / 2} \mid \sum_{i=1}^{n} Z_{i}\left\{I\left(Z_{i} \leq Q_{0}(\lambda)+n^{-1 / 2} t\right)-I\left(Z_{i} \leq Q_{0}(\lambda)\right)\right\} \\
& \quad-\left\{\mu\left(Q_{0}(\lambda)+n^{-1 / 2} t\right)-\mu\left(Q_{0}(\lambda)\right)\right\} \mid \\
& =n^{-1 / 2}\left|\sum_{i=1}^{n}\left(\tilde{V}_{i}(t)-\tilde{\nu}_{n}(t)\right)\right|
\end{aligned}
$$

and

$$
\tilde{V}_{i}(t)=Z_{i} I\left(Q_{0}(\lambda)<Z_{i} \leq Q_{0}(\lambda)+n^{-1 / 2} t\right) \text { and } \dot{\nu}_{n}(t)=E\left(\dot{V}_{i}(t)\right) \text {. }
$$


Now, for each $\epsilon>0$

$$
\begin{gathered}
P\left\{\left(n^{-1 / 2} \sum_{i=1}^{n}\left(\tilde{V}_{i}(t)-\tilde{V}_{n}(t)\right)\right)^{2}>\epsilon^{2}\right\} \\
\leq \frac{1}{\epsilon^{2}} \frac{1}{n} E\left(\sum_{i=1}^{n}\left(\tilde{V}_{i}(t)-\tilde{\nu}_{n}(t)\right)\right)^{2} \\
=\frac{1}{\epsilon^{2}} \frac{1}{n} \sum_{i=1}^{n} E\left(\tilde{V}_{i}(t)-\tilde{\nu}_{n}(t)\right)^{2} \\
=\frac{1}{\epsilon^{2}}\left\{E\left(\tilde{V}_{1}(t)\right)^{2}-\left(\dot{\nu}_{n}(t)\right)^{2}\right\}
\end{gathered}
$$

By continuity of fo each term inside the above bracket goes to zero as $n \rightarrow \infty$ for each $t$. For the tightness, we use the same argument as in Lemma 2.4.3. So, it is necessary to show that for all $\epsilon>0 \exists \delta>0$ such that for each $s \in \mathcal{N}(b)$

$$
\underset{n}{\limsup P} P\left(\sup _{|t-\infty| \leq \delta}\left|T_{n}^{0}(t ; \lambda)-T_{n}^{0}(s ; \lambda)\right|>2 \epsilon\right)<\epsilon .
$$

In this case we have

$$
\begin{aligned}
& \left|\tilde{T}_{n}^{0}(t ; \lambda)-\tilde{T}_{n}^{0}(s ; \lambda)\right| \\
& \leq n^{-1 / 2} \sum_{i=1}^{n}\left|Z_{i}\left\{I\left(Z_{i} \leq Q_{0}(\lambda)+n^{-1 / 2} t\right)-I\left(Z_{i} \leq Q_{0}(\lambda)+n^{-1 / 2} s\right)\right\}\right| \\
& \quad+n^{1 / 2}\left|\mu\left(Q_{0}(\lambda)+n^{-1 / 2} t\right)-\mu\left(Q_{0}(\lambda)+n^{-1 / 2} s\right)\right|
\end{aligned}
$$

By the monotone property of the indicator function with the relation (2.74), the above expression is bounded above by

$$
\begin{aligned}
& n^{-1 / 2} \sum_{i=1}^{n}\left|Z_{i}\right|\left\{I\left(Z_{i} \leq Q_{0}(\lambda)+n^{-1 / 2}+n^{-1 / 2} \delta\right)\right. \\
& \left.-I\left(Z_{i} \leq Q_{0}(\lambda)+n^{-1 / 2} s-n^{-1 / 2} \delta\right)\right\} \\
& +n^{1 / 2}\left|\mu\left(Q_{0}(\lambda)+n^{-1 / 2} t\right)-\mu\left(Q_{0}(\lambda)+n^{-1 / 2} s\right)\right| \\
& =J_{11}+J_{12} .
\end{aligned}
$$


By similar argument as $(2.106)$ and $(2.107) J_{12}=o(1)$. We only need to show that $J_{11}=\operatorname{op}(1)$. As we see, $J_{11}$ does not depend on $t$. We use Chebyshev inequality to show that it converges to zero in probability. Let us define

$$
\mu^{*}(x)=E\left[\left|Z_{i}\right| I\left(Z_{i} \leq x\right)\right] .
$$

Then,

$$
\begin{aligned}
J_{11}= & n^{-1 / 2} \sum_{i=1}^{n}\left\{|| Z_{i} \mid I\left(Z_{i} \leq Q_{0}(\lambda)+n^{-1 / 2} s+n^{-1 / 2} \delta\right)-\mu^{-}\left(Q_{0}(\lambda)+n^{-1 / 2} s+n^{-1 / 2} \delta\right)\right] \\
& \left.-\left[\left|Z_{i}\right| I\left(Z_{i} \leq Q_{0}(\lambda)+n^{-1 / 2} s-n^{-1 / 2} \delta\right)-\mu^{*}\left(Q_{0}(\lambda)+n^{-1 / 2} s-n^{-1 / 2} \delta\right)\right]\right\} \\
& +n^{1 / 2}\left\{\mu^{*}\left(Q_{0}(\lambda)+n^{-1 / 2} s+n^{-1 / 2} \delta\right)-\mu^{*}\left(Q_{0}(\lambda)+n^{-1 / 2} s-n^{-1 / 2} \delta\right)\right\}
\end{aligned}
$$

The second term of R.H.S of $J_{11}$ is less than $\epsilon$ for sufficiently small $\delta$ by the mean value Theorem. The first part is op $(1)$ by the argument similar to the proof of $J_{1}$ for fixed $t$ of Lemma 2.4.3. This ends the pronf of Lemma 2.4.6.

Before stating the next Lemma we consider the following processes,

$$
U_{n}(t)=n^{-1} \sum_{i=1}^{n} I\left(Z_{i} \leq Q_{0}(\lambda)+n^{-1 / 2} t\right)
$$

and

$$
U_{n}^{0}(t)=U_{n}(t)-E\left[U_{n}(t)\right]=U_{n}(t)-F_{0}\left(Q_{.1}(\lambda)+n^{-1 / 2} t\right) .
$$

Then,

Lemma 2.4.7 Assume that the condition FI hold. Then for every $b>0$

$$
\sup _{|x| \leq b}\left|U_{n}(t)-\lambda\right| \stackrel{P}{\rightarrow} 0
$$

Proof:

$$
\sup _{|l| \leq b}\left|U_{n}(t)-\lambda\right|
$$




$$
\begin{aligned}
& =\sup _{|k| \leq t}\left|n^{-1} \sum_{i=1}^{n}\left(I\left(Z_{i} \leq Q_{0}(\lambda)+n^{-1 / 2} t\right)-\lambda\right)\right| \\
& =\sup _{|k| \leq b} n^{-1}\left|\sum_{i=1}^{n}\right|\left(I\left(Z_{i} \leq Q_{0}(\lambda)+n^{-1 / 2} t\right)-F_{0}\left(Q_{0}(\lambda)+n^{-1 / 2} t\right)\right. \\
& \left.+F_{0}\left(Q_{0}(\lambda)+n^{-1 / 2} t\right)-\lambda\right] \mid \\
& \leq \sup _{|1| \leq b} n^{-1} \mid \sum_{i=1}^{n}\left[\left(I\left(Z_{i} \leq Q_{0}(\lambda)+n^{-1 / 2} t\right)-F_{0}\left(Q_{0}(\lambda)+n^{-1 / 2} t\right)\right] \mid\right. \\
& +\sup _{|k| \leq b}\left|F_{0}\left(Q_{0}(\lambda)+n^{-1 / 2} t\right)-\lambda\right| \\
& =I_{1}+I_{2} \text {. }
\end{aligned}
$$

Under the condition Fl we get $I_{2} \rightarrow 0$ as $n \rightarrow \infty$. F c $I_{2}$, we follow the same argument as in Lemma 2.4 .3 along with the weak law of large number.

Now, we change the process $U_{n}(t)$ to $U_{n}^{*}(t)$ as

$$
U_{n}^{*}(t)=n^{-1} \sum_{i=1}^{n} I\left(Q_{0}\left(\lambda_{1}\right)+n^{-1 / 2} t<Z_{i} \leq Q_{0}\left(\lambda_{2}\right)+n^{-1 / 2} t\right) .
$$

Then, if $f_{0}$ is continuous in the neighborhood of $Q_{0}\left(\lambda_{1}\right)$ and $Q_{0}\left(\lambda_{2}\right)$, Lemma 2.4.7 implies

$$
\sup _{|x| \leq b}\left|U_{n}^{*}(t)-\left(\lambda_{2}-\lambda_{1}\right)\right|=o_{P}(1)
$$

This result together with the structure of $A$ implies

$$
n^{-1} 1^{\prime} A 1=\left(\lambda_{2}-\lambda_{1}\right)+O_{P}(1) \text { as } n \rightarrow \infty \text {. }
$$

Further, note that

$$
\begin{aligned}
n^{-1 / 2} 1^{\prime} A Z= & n^{-1 / 2} \sum_{i=1}^{n} Z_{i}\left\{I\left(Z_{i} \leq Q_{0}\left(\lambda_{2}\right)+n^{-1 / 2}\left[n^{1 / 2}\left(\bar{Q}_{n}\left(\lambda_{2}\right)-\beta_{0}\left(\lambda_{2}\right)\right)\right]\right)\right. \\
& \left.-I\left(Z_{i} \leq Q_{0}\left(\lambda_{1}\right)+n^{-1 / 2}\left[n^{1 / 2}\left(Q_{n}\left(\lambda_{1}\right)-\beta_{0}\left(\lambda_{1}\right)\right)\right]\right)\right\} . \quad(2.1
\end{aligned}
$$

Now, using Lemma 2.4 .6 for the first and second term of the R.H.S of (2.125), we have

$$
n^{-1 / 2} 1^{\prime} A Z=T_{n}\left(\beta_{0}\left(\lambda_{1}\right) ; \lambda_{1}\right) Q_{0}\left(\lambda_{1}\right)-T_{n}\left(\beta_{0}\left(\lambda_{2}\right) ; \lambda_{2}\right) Q_{0}\left(\lambda_{2}\right)
$$




$$
+n^{-1 / 2} \sum_{i=1}^{n} Z_{i} I\left(Q_{0}\left(\lambda_{1}\right)<Z_{i} \leq Q_{0}\left(\lambda_{2}\right)\right)+o_{P}(1)
$$

and by the definition of $T_{n}\left(\beta_{0}(\lambda) ; \lambda\right)$ (given in (2.59)) we get

$$
n^{-1 / 2} 1^{\prime} A Z=n^{-1 / 2} \sum_{i=1}^{n}\left(\phi\left(Z_{i}\right)-\gamma\right)+o p(1) .
$$

Finally, we have

$$
\left(1^{\prime} A 1\right)^{-1} 1^{\prime} A Z=\left(1^{\prime} A 1\right)^{-1} 1^{\prime} A Y-\beta_{0}
$$

or

$$
\left(n^{-1} 1^{\prime} A 1\right)^{-1} n^{-1 / 2} 1^{\prime} A Z=n^{1 / 2}\left(\bar{\beta}_{0 n}\left(\lambda_{1}, \lambda_{2}\right)-\beta_{0}\right)
$$

and as a result

$$
n^{1 / 2}\left(\bar{\beta}_{0 n}\left(\lambda_{1}, \lambda_{2}\right)-\beta_{0}\right)=\left(\lambda_{2}-\lambda_{1}\right)^{-1} n^{-1 / 2} \sum_{i=1}^{n}\left(\phi\left(Z_{i}\right)-\gamma\right)+o_{P}(1) .
$$

This completes the proof of part (i) of Theorem 2.4.5. The proof of the second part of this theorem follows by the C.L.T property of i.i.d random variables.

Theorem 2.4.3 states that TLSE converges in probability to a normal distribution with variance $\sigma^{2}\left(\lambda_{1}, \lambda_{2}\right)$. Now, we wish to determine a consistent estimator of $\sigma^{2}\left(\lambda_{1}, \lambda_{2}\right)$ which will be helpful for future inference ie.g. testing hypotheses, confidence intervals). Let $Y_{1}, Y_{2} \ldots, Y_{n}$ be a sample from the location model (2.45). We may define an estimate of $\sigma^{2}\left(\lambda_{1}, \lambda_{2}\right)$ by

$$
\begin{aligned}
S_{n}^{2}\left(\lambda_{1}, \lambda_{2}\right)= & \left(\lambda_{2}-\lambda_{1}\right)^{-2}\left\{(n-1)^{-i} \sum_{i=1}^{n}\left(Y_{i}-\delta_{n}\right)^{2} I\left(\bar{Q}_{n}\left(\lambda_{1}\right)<Y_{i} \leq Q_{n}\left(\lambda_{2}\right)\right)\right. \\
& +\lambda_{1}\left(\bar{Q}_{n}\left(\lambda_{1}\right)-\delta_{n}\right)^{2}+\left(1-\lambda_{2}\right)\left(\bar{Q}_{n}\left(\lambda_{2}\right)-\delta_{n}\right)^{2} \\
& \left.-\left[\lambda_{1}\left(\bar{Q}_{n}\left(\lambda_{1}\right)-\delta_{n}\right)+\left(1-\lambda_{2}\right)\left(\bar{Q}_{n}\left(\lambda_{2}\right)-\delta_{n}\right)\right]^{2}\right\}
\end{aligned}
$$

where $\bar{Q}_{n}\left(\lambda_{i}\right),(i=1,2)$ is the sample quantile with order $\lambda_{i}$ and

$$
\delta_{n}=\left[n\left(\lambda_{2}-\lambda_{1}\right)\right]^{-1} \sum_{i=1}^{n} Y_{i} I\left(\bar{Q}_{n}\left(\lambda_{1}\right)<Y_{i} \leq \bar{Q}_{n}\left(\lambda_{2}\right)\right)
$$

Then, 
Theorem 2.4.8 Let the condition of Theorem 2.4.5 hold. Then

$$
S_{n}^{2}\left(\lambda_{1}, \lambda_{2}\right) \stackrel{P}{\rightarrow} \sigma^{2}\left(\lambda_{1}, \lambda_{2}\right)
$$

Proof:

To prove this theorem first we shall show that

$$
\boldsymbol{\delta}_{n} \stackrel{P}{\rightarrow} \delta
$$

where

$$
\delta=\left(\lambda_{2}-\lambda_{1}\right)^{-1} \int_{Q\left(\lambda_{1}\right)}^{Q\left(\lambda_{2}\right)} Y d F(y)=\beta_{0}+\delta_{0}
$$

and $\delta_{0}$ defined in (2.99). Equivalently, it is necessary to show that

$$
\delta_{0 n} \stackrel{P}{\rightarrow} \delta_{0}
$$

where

$$
\delta_{0 n}=\left[n\left(\lambda_{2}-\lambda_{1}\right)\right]^{-1} \sum_{i=1}^{n} Z_{i} I\left(\bar{Q}_{0 n}\left(\lambda_{1}\right)<Z_{i} \leq \bar{Q}_{0 n}\left(\lambda_{2}\right)\right) .
$$

We need the following lemma to prove (2.136).

Lemma 2.4.9 Let $\xi_{1}, \xi_{2}, \ldots, \xi_{n}$ be i.i.d r.v's with cdf $F$ and let $g$ be a measurable function such that $g(\cdot) \geq 0$ and $E\left(g\left(\xi_{1}\right)\right)<\infty$. Define

$$
W_{n}(x)=n^{-1} \sum_{i=1}^{n}\left[g\left(\xi_{i}\right) I\left(\xi_{i} \leq x\right)-\nu(x)\right]
$$

where

$$
\nu(x)=E\left[g\left(\xi_{1}\right) I\left(\xi_{1} \leq x\right)\right]
$$

Then

$$
\sup _{-\infty<x<\infty}\left|W_{n}(x)\right| \rightarrow 0 \text { a.s. }
$$

We shall prove this lemma at the end of this chapter. 
Now, we are going to show (2.136)

$$
\begin{aligned}
\left|\delta_{0 n}-\delta_{0}\right|= & c\left|n^{-1} \sum_{i=1}^{n}\left\{Z_{i} I\left(\bar{Q}_{0 n}\left(\lambda_{1}\right)<Z_{i} \leq \bar{Q}_{0 n}\left(\lambda_{2}\right)\right)-\int_{Q_{0}\left(\lambda_{1}\right)}^{Q_{0}\left(\lambda_{2}\right)} Z d F_{0}(z)\right\}\right| \\
\leq & c\left|n^{-1} \sum_{i=1}^{n}\left\{Z_{i} I\left(Z_{i} \leq \bar{Q}_{0_{n}}\left(\lambda_{2}\right)\right)-\nu\left(Q_{0}\left(\lambda_{2}\right)\right)\right\}\right| \\
& +c\left|n^{-1} \sum_{i=1}^{n}\left\{Z_{i} I\left(Z_{i} \leq \bar{Q}_{0 n}\left(\lambda_{1}\right)\right)-\nu\left(Q_{0}\left(\lambda_{1}\right)\right)\right\}\right| \\
= & c\left(I_{1}+I_{2}\right) .
\end{aligned}
$$

Thus, we have to show that $I_{1}=o p(1)=I_{2}$. Let us write the r.v $Z_{1}$ as

$$
Z_{i}=Z_{i}^{+}-Z_{i}^{-}
$$

and define

$$
W_{n}^{ \pm}(\lambda)=n^{-1} \sum_{i=1}^{n} Z_{i}^{ \pm} I\left(Z_{i} \leq \bar{Q}_{0 n}(\lambda)\right)-\nu^{ \pm}\left(Q_{0}(\lambda)\right)
$$

It is necessary to show that $\left|W_{n}^{+}(\lambda)\right|=o_{P}(1)=\left|W_{n}^{-}(\lambda)\right|$, where

$$
\begin{aligned}
\left|W_{n}^{+}(\lambda)\right|= & \left|n^{-1} \sum_{i=1}^{n} Z_{i}^{+} I\left(Z_{i} \leq \bar{Q}_{0_{n}}(\lambda)\right)-\nu^{+}\left(Q_{0}(\lambda)\right)\right| \\
\leq & \left|n^{-1} \sum_{i=1}^{n} Z_{i}^{+} I\left(Z_{i} \leq \bar{Q}_{0_{n}}(\lambda)\right)-\nu^{+}\left(\bar{Q}_{0 n}(\lambda)\right)\right| \\
& \quad+\left|\nu^{+}\left(\bar{Q}_{0 n}(\lambda)\right)-\nu^{+}\left(Q_{0}(\lambda)\right)\right| .
\end{aligned}
$$

The first term of R.H.S of (2.144) goes to zero a.s. by Lemma 2.4.9. The second term also converges to zero as $n \rightarrow 0$ by consistency of sample quantile given in Lemma 2.4.4. By the same argument we get that $\left|W_{n}^{-}(\lambda)\right| \rightarrow 0$ as $n \rightarrow \infty$ and then,

$$
\left|W_{n}(\lambda)\right| \rightarrow 0 \quad \text { a.s. }
$$

Therefore we get that $I_{1}=o_{P}(1)=I_{2}$. Using similar argument we have

$$
\mid n^{-1} \sum_{i=1}^{n}\left\{Z_{i}^{2} I\left(\bar{Q}_{0 n}\left(\lambda_{1}\right)<Z_{i} \leq \bar{Q}_{0 n}\left(\lambda_{2}\right)\right)-\int_{Q_{0}\left(\lambda_{1}\right)}^{Q_{0}\left(\lambda_{2}\right)} Z^{2} d F_{0}(z) \mid=o_{P}(1)\right.
$$


Finally, by Lemma 2.4.4 we have

$$
\bar{Q}_{\text {on }}(\lambda) \stackrel{P}{\rightarrow} Q_{0}(\lambda)
$$

Combining all the above results we claim the proof of Theorem 2.4.8.

Proor of Lemma 2.4.9:

Let $0<M<\infty$ be an arbitrary fixed real number. Then, ve may write

$$
\begin{aligned}
\sup _{-\infty<x<\infty}\left|W_{n}(x)\right| \leq & \sup _{-\infty<x<-M}\left|W_{n}(x)\right|+\sup _{-M \leq x \leq M}\left|W_{n}(x)\right| \\
& +\sup _{M<x<\infty}\left|W_{n}(x)\right| \\
& =I_{1}+I_{2}+I_{3} .
\end{aligned}
$$

We have to show that each term of R.H.S of above inequality goes to zero. First, we are going to show that $I_{2} \rightarrow 0$ a.s. For this reason ve partition the interval $[-M, M]$ into $k$ parts. Then, for each $x$,

$$
\frac{j}{k} 2 M \leq x \leq \frac{j+1}{k} 2 M
$$

we have

$$
\begin{gathered}
W_{n}\left(\frac{j}{k} 2 M\right)+\nu\left(\frac{j}{k} 2 M\right)-\nu\left(\frac{j+1}{k} 2 M\right) \leq W_{n}(x) \\
\quad \leq W_{n}\left(\frac{j+1}{k} 2 M\right)+\nu\left(\frac{j+1}{k} 2 M\right)-\nu\left(\frac{j}{k} 2 M\right)
\end{gathered}
$$

which implies for each $x \in\left[\frac{i}{k} 2 M, \frac{i+1}{k} 2 M\right]$,

$$
\left|W_{n}(x)\right| \leq 2 \max _{1 \leq j \leq k}\left|W_{n}\left(\frac{j}{k} 2 M\right)\right|+\max _{1 \leq j \leq k}\left|\nu\left(\frac{j+1}{k} 2 M\right)-\nu\left(\frac{j}{k} 2 M\right)\right| .
$$

Thus,

$$
\begin{aligned}
\sup _{-M \leq x \leq M}\left|W_{n}(x)\right| & \leq 2 \max _{1 \leq j \leq k}\left|W_{n}\left(\frac{j}{k} 2 M\right)\right|+\max _{1 \leq j \leq k}\left|\nu\left(\frac{j+1}{k} 2 M\right)-\nu\left(\frac{j}{k} 2 M\right)\right| \\
& =I_{31}+I_{22} .
\end{aligned}
$$


By S.L.L.N, for each $1 \leq j \leq k$ we have $I_{21} \rightarrow 0$ a.s. We get the same result for the maximum over $j$ since $M$ and $k$ are fixed. For $I_{22}, \forall \in>0$ there exist $M=\left(M_{\mathrm{e}}\right), k=k_{\mathrm{e}}(M)$ and $\delta>0$ such that $\frac{2 M}{k}<\delta$. Then,

$$
\max _{1 \leq j \leq k}\left|\nu\left(\frac{j+1}{k} 2 M\right)-\nu\left(\frac{j}{k} 2 M\right)\right|<\epsilon .
$$

Now, we consider $I_{1}$

$$
\begin{aligned}
I_{1} & =\sup _{-\infty<x<-M}\left|W_{n}(x)\right| \\
& \leq \sup _{-\infty<x<-M} n^{-1} \sum_{i=1}^{n} g\left(\xi_{i}\right) I\left(\xi_{i} \leq x\right)+\sup _{-\infty<x<-M} \nu(x) \\
& =I_{12}+I_{12} .
\end{aligned}
$$

But, for each $\epsilon>0 \exists M_{\epsilon}$ such that

$$
I_{12} \leq \int_{-\infty}^{-M} g(\xi) d F(\xi)<\epsilon \quad \forall M>M_{c} .
$$

For, $I_{11}$ we have

$$
\begin{aligned}
I_{11} & =\sup _{-\infty<x<-M} n^{-1} \sum_{i=1}^{n} g\left(\xi_{i}\right) I\left(\xi_{i} \leq x\right) \\
& \leq n^{-1} \sum_{i=1}^{n} g\left(\xi_{i}\right) I\left(\xi_{i} \leq-M\right) \rightarrow \nu(-M) \quad \text { a.s. }
\end{aligned}
$$

From (2.155), we have $\nu(-M)<\epsilon \quad \forall M>M_{\epsilon}$. Therefore, we get the result for $I_{1}$. The last one which must be checked is $I_{3}$ where,

$$
\begin{aligned}
I_{3}= & \sup _{M<x<\infty}\left|W_{n}(x)\right| \\
= & \sup _{M<x<\infty}\left|n^{-1} \sum_{i=1}^{n} g\left(\xi_{i}\right) I\left(\xi_{i} \leq x\right)-\nu(x)\right| \\
= & \sup _{M<x<\infty}\left|n^{-1} \sum_{i=1}^{n}\left\{g\left(\xi_{i}\right)\left(1-I\left(\xi_{i}>x\right)\right)-E\left[g\left(\xi_{i}\right)\left(1-I\left(\xi_{i}>x\right)\right)\right]\right\}\right| \\
\leq & \left|n^{-1} \sum_{i=1}^{n}\left\{g\left(\xi_{i}\right)-E\left[g\left(\xi_{i}\right)\right]\right\}\right|+\sup _{M<x<\infty} n^{-1} \sum_{i=1}^{n} g\left(\xi_{i}\right) I\left(\xi_{i}>x\right) \\
& +\sup _{M<x<\infty} E\left[g\left(\xi_{i}\right) I\left(\xi_{i}>x\right)\right] \\
= & I_{31}+I_{32}+I_{33} .
\end{aligned}
$$


CHAPTER 2. LOCATION AND SCALE MODEL

36

By S.L.L.N we obtain that $I_{31} \rightarrow 0$ ass. For $I_{32}$ we have $\forall \epsilon>0 \exists M_{\text {c }}$ such that

$$
\begin{aligned}
I_{32} & =\sup _{M<x<\infty} n^{-1} \sum_{i=1}^{n} g\left(\xi_{i}\right) I\left(\xi_{i}>x\right) \\
& \leq n^{-1} \sum_{i=1}^{n} g\left(\xi_{i}\right) I\left(\xi_{i}>M\right) \rightarrow \int_{M}^{\infty} g(\xi) d F(\xi) \quad \text { ass. } \\
& <\epsilon \forall M>M_{\mathrm{c}} .
\end{aligned}
$$

We also get the same result for $I_{33}$. ie, $I_{33}<\epsilon \forall M>M_{\ell}$ which implies that $I_{3} \rightarrow 0$ ass. as $n \rightarrow \infty$ and the proof of the Lemma 2.4 .9 is complete. 


\section{Chapter 3}

\section{Regression Model}

\subsection{Introduction}

Linear combination of order statistics or L-estimators plays an extremely important role in the development of robust methods for location parameters. An important precursor to the trimmed L-estimator is the L-estimator consisting of a few fixed number of order statistics, for example, the median, trimmed, and Gastwirth's estimator. In the last chapter, we have studied L-estimators based on a few selected optimum sample quantiles for some parametric models to evaluate the asymptotic relative efficiencies of such procedures. Hogg (1974) preferred trimmed means and Stigler (1977) concluded that the $10 \%$ trimmed mean emerges as the victor in analysing data from $18^{\text {th }}$ and $19^{\text {th }}$ century experiments designed to measure some physical constants. As a first attempt, Bickel (1973) considered a class of onestep L-estimators of regression coefficients in a linear model depending on a "preliminary estimator". Although these estimators have good asymptotic properties, they are computationally complex and apparently not invariant to reparameterization. The object of this chapter is to consider the well accepted definition of regression quantiles of Koenker and Bassett (1978) in the linear model and pursue the question of L-estimation based on a few selected regression quantiles and trimmed least 
squares estimation. The results about the trimmed least square estimator in this chapter are obtained by improving the conditions initially given by Ruppert and Carroll (1980) and Jurekkova (1984) and are new.

In section 3.2 we present the definition of regression quantiles and its properties in detail as developed by Koenker and Bassett (1978) and Bassett and Koenker (1978). Other sections that follow are new results. Section 3.3 describes the Lestimation of regression parameters based on few selected regression quantiles for some known error distributions in order to assess the asymptotic relative efficiency (ARE) properties. Section 3.4 contains the trimmed L-estimators while section 3.5 concludes with some improved estimators of regression parameters on the basis of trimmed L-estimators combining the idea of robust estimation and Stein-type estimation using the preliminary test approach of Saleh and Sen (1978-86).

\subsection{Regression Quantiles and Their Properties}

Consider the linear model

$$
Y_{n \times 1}=\beta_{0} 1_{n}+X_{n \times p} \beta_{p \times 1}+Z_{n \times 1}
$$

where $Z_{n \times 1}=\left(Z_{1}, \cdots, Z_{n}\right)^{\prime}$ consists of i.i.d. r.v's with a continuous $c d f F_{0}(z)$ and $p d f f_{0}(z), z \in \mathbb{R}$. It is well-known that the least squares estimator (LSE) of $\beta^{*}=\left(\beta_{0}, \beta^{\prime}\right)^{\prime}$ is given by

$$
\tilde{\beta}_{n}^{* L}=\left(D_{n}^{\prime} D_{n}\right)^{-1} D_{n}^{\prime} Y
$$

where $D_{n}=\left(1_{n} \mid X\right)$ is the design matrix and the quadratic estimator of variance is given by

$$
S^{2}=\frac{\left(Y-\tilde{\beta}_{0}^{L} 1_{n}-X \tilde{\beta}_{n}^{L}\right)^{\prime}\left(Y-\tilde{\beta}_{0}^{L} 1_{n}-X \tilde{\beta}_{n}^{L}\right)}{n-p-1}
$$


It is further well-known that the LSE of $\boldsymbol{\beta}^{*}$ is not robust. A natural curiosity is to develop analogues of linear combination of order statistics and simplified quantile estimators for the linear model. Bickel (1973) considered ordering of the residuals based on a preliminary estimator. But his method is computationally complicated and needs basically iteratively re-weighted least squares techniques.

However, Koenker and Bassett (1978) extended a well-accepted definition of sample quantiles in linear models which they call regression quantiles. We shall now devote our attention to this concept of quantiles, present their properties as in Koenker and Bassett (1978) and apply them to the estimation of regression parameters.

Definition 3.2.1 regression quantiles of order $\lambda$ is the solution of the minimization problem

$$
\min _{b \in \mathbb{R}^{p+1}}\left[\sum_{j \in J} \lambda\left|y_{j}-d_{j}^{\prime} b\right|+\sum_{j \in J}(1-\lambda)\left|y_{j}-d_{j}^{\prime} b\right|\right]
$$

where $J=\left\{j: y_{j}-d_{j}^{\prime} b \geq 0\right\}$ and $d_{j}$ is the $j^{\text {th }}$ row of $D_{n}$ the design matrix. For $p=0$ and $d_{j}=1$ the minimization problem yields $b_{0}$ equal to the sample quantile of order $\lambda$, while $\lambda=1 / 2$ yields $b_{0}$ to be the sample median. Thus, the median is the least absolute error estimator (LAE) of $\beta_{0}$.

Let $\hat{\mathbf{B}}(\lambda)$ be the solution sets consisting of $\left(\hat{\boldsymbol{\beta}}_{0}(\lambda), \hat{\boldsymbol{\beta}}_{n}^{\prime}(\lambda)\right)^{\prime}$ of the regr.sssion quantiles by minimising (3.4). Also, let $\mathcal{H}$ denote the $(p+1)$-element subsets of $I=\{1,2, \cdots, n\}$. There are $\left(\begin{array}{c}n \\ p+1\end{array}\right)$ possible subsets in $\mathcal{H}$. Elements $h \in \mathcal{H}$ have complements $\bar{h}=I-h$ and $h$ and $\bar{h}$ partition the vector $Y$ as well as the design matrix $D_{n}$. Thus, the notation $Y(h)$ stands for the $(p+1)$-element vector of $y$ 's from $Y$, i.e. $\left\{y_{j}, j \in h\right\}$ while $D_{n}(\bar{h})$ stands for a $(n-p-1) \times(p+1)$ matrix with rows $\left\{\left(1, x_{j}\right) \mid j \in \bar{h}\right\}$. Finally, let

$$
\mathbf{H}=\left\{h \in \mathcal{H} \mid \operatorname{rank} D_{n}(h)=p+1\right\} .
$$

Then, the following theorem gives the form of the solution of (3.4). 
Theorem 3.2.2 If the rank of $D_{n}$ is $p+1$, then the set of regression quantiles $\hat{\mathbf{B}}(\lambda)$ has at least one element of the form

$$
\hat{\boldsymbol{\beta}}_{n}^{*}(\lambda)=D_{n}(h)^{-1} \boldsymbol{Y}(h)
$$

for some $h \in \mathcal{H}$. Moreover, $\hat{\mathbf{B}}(\lambda)$ is the convex hull of all solutions of the form (3.6).

The proof of Theorem 3.2.2 follows from the linear programing formulation of the minimization problem as given below:

$$
\min \left[\lambda 1_{n}^{\prime} r^{+}+(1-\lambda) 1_{n}^{\prime} r^{-}\right], 1_{n}=(1, \ldots, 1)^{\prime}
$$

$$
\text { subject to } Y=D_{n} b+r^{+}-r^{-}, \quad\left(b, r^{+}, r^{-}\right) \in \mathbb{R}^{p+1} \times \mathbb{R}_{+}^{n} \times \mathbb{R}_{+}^{n} \text {. }
$$

For example see Abdelmalek (1974).

Sample quentiles in the location model are identified with a single order statistic in a sample. Theorem 3.2.2 generasized this feature to regression quantiles where normals to hyperplanes defined by subset of $(p+1)$ observations play the role of order statistics.

The following Theorems describe various other properties of regression quantiles (RQs') given in Koenker and Bassett (1978).

Theorem 3.2.3 If $\hat{\boldsymbol{B}}_{n}^{*}\left(\lambda, Y, D_{n}\right) \in \hat{\mathbf{B}}\left(\lambda, Y, D_{n}\right)$ then the following are elements of the specified transformed problems:

(i) $\quad \hat{\beta}_{n}^{*}\left(\lambda, c Y, D_{n}\right)=c \hat{\beta}_{n}^{*}\left(\lambda, Y, D_{n}\right), \quad c \in[0, \infty)$.

(ii) $\quad \hat{\beta}_{n}^{*}\left(1-\lambda, d Y, D_{n}\right)=d \hat{\beta}_{n}^{*}\left(\lambda, Y, D_{n}\right), \quad d \in(-\infty, 0]$.

(iii) $\hat{\boldsymbol{\beta}}_{n}^{*}\left(\lambda, Y+D_{n} \gamma, D_{n}\right)=\hat{\beta}_{n}^{*}\left(\lambda, Y, D_{n}\right)+\gamma, \quad \gamma \in \mathbb{R}^{p+1}$

(iv) $\hat{\beta}_{n}^{*}\left(\lambda, Y, D_{n} C\right)=C^{-1} \hat{\beta}_{n}^{*}\left(\lambda, Y, D_{n}\right), \quad C_{p+1 \times p+1}$ non-singular matrix.

The following theorem gives the conditions when RQ's are unique. 
Theorem 3.2.4 If the error distribution $F_{0}(z)$ is continuous, then with probability one: $\hat{\beta}_{n}^{*}(\lambda)=D_{n}(h)^{-1} Y(h)$ is a unique solution if and only if

$$
(\lambda-1) 1_{p+1}^{\prime}<\sum_{j \neq h}\left[1 / 2-1 / 2 \operatorname{sgn}\left(y_{j}-d_{j}^{\prime} \hat{\beta}_{n}^{*}\right)-\lambda\right] d_{j}^{\prime} D_{n}(h)^{-1}<\lambda 1_{p+1}^{\prime}
$$

where $1_{p+1}$ is a vector of ones.

The next Theorem states the relationship of the number residual errors $\hat{u}_{j}=$ $y_{j}-\alpha_{j}^{*} \hat{\beta}_{n}^{*}(\lambda), j=1, \cdots, n$ that are positive, negative and zero with that of $n \lambda$.

Theorem 3.2.5 Let $\hat{u}=\left(\hat{u}_{1}, \cdots, \hat{u}_{n}\right)^{\prime}$ and $P(\hat{u}), N(\hat{u})$ and $Z(\hat{u})$ be the number of positive, negative and zero residuals. Then,

$$
N(\hat{\boldsymbol{u}}) \leq n \lambda \leq n-P(\hat{\boldsymbol{u}})=N(\hat{\boldsymbol{u}})+Z(\dot{\boldsymbol{u}})
$$

for every $\left.\hat{\boldsymbol{\beta}}_{n}^{-}(\lambda) \in \hat{\mathbf{B}}^{(} \lambda\right)$. If $\hat{\boldsymbol{\beta}}_{n}^{*}(\lambda)$ is unique, i.e., $\hat{\boldsymbol{\beta}}_{n}^{-}(\lambda)=\hat{\mathbf{B}}(\lambda)$ then inequalities hold strictly.

The following theorem has the following geometric interpretation: Consider a scatter diagram of sample observations in $\mathbb{R}^{2}$ with $\lambda^{\text {th }}$-regression quantiles slicing through the scatter. Now, consider the effect (on the position of the $\lambda$-regression quantile) of moving observations up or down in the scatter. The result states that as long as these movements leave observations on the same side of the original line, its position is unaffected.

Theorem 3.2.6 If $\hat{\beta}_{n}^{*}(\lambda) \in \hat{\mathbf{B}}\left(\lambda, Y, D_{n}\right)$, then $\hat{\beta}_{n}^{*}(\lambda) \in \hat{\mathbf{B}}\left(\lambda, D_{n} \hat{\beta}_{n}^{*}+A \hat{u}, D_{n}\right)$ where $\hat{u}=\boldsymbol{Y}-D_{n} \hat{\beta}_{n}^{*}$ and $A$ is any $n \times n$ diagonal matrix with nonnegative elements.

Before stating the next Theorem it is necessary to consider the following conditions on the distribution function and the design matrix.

A0) $\quad F_{0}$ is continuous and has continuous density $f_{0}$ in the neighborhood of $Q_{0}\left(\lambda_{i}\right)$ when $q_{0}\left(\lambda_{i}\right)>0 \quad i=1, \ldots k$ 


$$
n^{-1 / 2} \max _{i, j}\left|d_{i j}\right|=o(1)
$$

$$
\lim _{n \rightarrow \infty} \Sigma_{n}=\lim _{n \rightarrow \infty} n^{-1} D_{n}^{\prime} D_{n}=\Sigma \text {. p.d. }
$$

Then,

Theorem 3.2.7 Let $\left(\hat{\boldsymbol{\beta}}_{n}^{*}\left(\lambda_{1}\right), \cdots, \hat{\beta}_{n}^{*}\left(\lambda_{k}\right)\right)$ with $0<\lambda_{2}<\cdots<\lambda_{k}<1$ for ficed $k(p+1 \leq k \leq n)$, be the sequence of unique RQ's from the model (9.1). Define

$$
\beta^{*}(\lambda)=\left(\beta_{0}+\sigma Q_{0}(\lambda), \beta_{1}, \cdots, \beta_{p}\right)
$$

Then

$$
n^{1 / 2}\left[\left(\hat{\beta}_{n}^{-}\left(\lambda_{1}\right)-\beta\left(\lambda_{1}\right)\right), \cdots,\left(\dot{\beta}_{n}^{*}\left(\lambda_{k}\right)-\beta\left(\lambda_{k}\right)\right)\right] \stackrel{D}{\rightarrow} \mathcal{N}_{k \times p+1}\left(0, \Omega \otimes \Sigma^{-1}\right)
$$

where

$$
\boldsymbol{\Omega}=\left(\left(\frac{\lambda_{i} \wedge \lambda_{j}-\lambda_{i} \lambda_{j}}{q_{0}\left(\lambda_{i}\right) q_{0}\left(\lambda_{j}\right)}\right)\right)
$$

We recognize that $\boldsymbol{\Omega}$ is the covariance matrix of $\boldsymbol{k}$ regression quantile with the spacing $\lambda=\left(\lambda_{1}, \cdots, \lambda_{k}\right)^{\prime}$. We will also prove this theorem via weighted empirical processes in section 3.4 .

Finally, we present the following theorem 3.2.8 which is the basis of studying the problem of estimation of the regression parameters based on a few selected "regression quantiles" in some known error distributions.

Theorem 3.2.8 Let $a(\lambda)=\left(a\left(\lambda_{1}\right), \ldots, a\left(\lambda_{k}\right)\right)^{\prime}$ be coefficients such that $\sum_{i=1}^{k} a\left(\lambda_{i}\right)=1$ and let $A 0-A 2$ hold. Then

$$
\dot{\beta}_{n}^{*}(\lambda)=\sum_{i=1}^{k} a\left(\lambda_{i}\right) \dot{\beta}_{n}^{*}\left(\lambda_{i}\right)
$$

is invariant to location, scale and reparameterization of design and $n^{1 / 2}\left(\tilde{\beta}_{n}^{*}(\lambda)-\right.$ $\left.\beta^{*}(\lambda)\right)$ converges in distribution to a $(p+1)$-variate Gaussian distribution with mean 0 and covariance matrix $a(\lambda) \operatorname{Ra}(\lambda) \Sigma^{-1}$. 


\subsection{L-estimation of the parameters of a linear model based on a few selected regression quan- tiles with known error distributions.}

In this section we consider the simplified L-estimation of the regression and scale parameters of a linear model based on a few selected regression quantiles.

Consider the linear model

$$
Y=D_{n} \beta^{*}+\sigma Z
$$

where $Z=\left(Z_{1}, \cdots, Z_{n}\right)^{\prime}$ consists of $i \cdot i \cdot d$. error variables with a continuous known $c d f F_{0}(z)$ and $p d f f_{0}(z), z \in \mathbb{R}$ and $\beta^{*}$ and $\sigma$ are the regression and scale parameters respectively. It is well-known that least squares estimation leads to the best linear unbiased estimator (BLUE) of $\beta^{*}$ given by (3.2) and the optimum quadratic estimator of $\sigma^{2}$ is given by (3.3). These estimators are not robust. Also the asymptotic efficiency of this BLUF tepends on the error distribution.

In this section, we introduce the estimation of $\left(\beta^{* \prime}, \sigma\right)^{\prime}$ based on a few selected regression quantiles (RQ) of Koenker and Bassett (1978) which will relate to simple robust extimators of $\beta^{*}$. This study will also extend the work of Ogawa (1951) on the estimation of location and scale model leading to simplified estimation of the regression and scale parameters of a linear model. Our program is to propose asymptotically best linear (in regression quantiles) unbiased estimators (ABLUE) of $\beta^{*}$ and $\sigma$ based on a few fixed, say $k,(p+2 \leq k<n)$ selected regression quantiles (RQ) when the error distribution is known. We shall also assess their asymptotic relative efficiency properties when the error distribution is the class of t-distributions.

For a given integer $k(p+2 \leq k<n)$, consider the spacing vector $\lambda=\left(\lambda_{1}, \cdots, \lambda_{k}\right)^{\prime}$ whose components satisfy the constraints $0<\lambda_{1}<\cdots<\lambda_{k}<1$. Now, by the 
minimisation problem (3.4), we obtain the $k$ RQ's given by the vector

$$
\hat{\beta}_{j n}(\lambda)=\left(\hat{\beta}_{j n}\left(\lambda_{1}\right), \cdots, \hat{\beta}_{j n}\left(\lambda_{k}\right)\right)^{\prime}, \quad j=0,1, \cdots, p
$$

Then, using Theorem 3.2.7, one obtains that the $k(p+1)$-dimensional random variable

$$
n^{1 / 2}\left(\left(\hat{\beta}_{0 n}(\lambda)-\beta_{0} 1_{k}-\sigma u\right)^{\prime},\left(\hat{\beta}_{1 n}(\lambda)-\beta_{1} 1_{k}\right)^{\prime}, \cdots,\left(\hat{\beta}_{p m}(\lambda)-\beta_{p} 1_{k}\right)^{\prime}\right)^{\prime}
$$

converges in law to the $k(p+1)$ dimensional normal distribution with mean 0 and covariance matrix $\sigma^{2}\left(\Sigma^{-1} \otimes \Omega\right)$ where $v=\left(u_{1}, \cdots, u_{k}\right)^{\prime}$ with $u_{j}=Q_{0}\left(\lambda_{j}\right), j=$ $1, \ldots, k, 1=(1, \cdots, 1)^{\prime}$, a k-tuple of ones, and $\Omega$ is given at (3.13).

\subsubsection{Joint Estimation of Regression and Scale Parameters}

In this subsection, we propose asymptotically best linear (in regression quantiles) unbiased estimators (ABLUE) of $\left(\beta^{* \prime}, \sigma\right)^{\prime}$. ABLUE of $\left(\beta_{0}, \beta_{1}, \cdots, \beta_{p}\right)^{\prime}$ and $\sigma$ can be obtained by minimising the quadratic form using (3.17) as

$$
\sum_{i=0}^{p} \sum_{j=0}^{p} \sigma_{i+1, j+1}\left(\hat{\theta}_{i n}-\theta_{i}\right)^{\prime} \Omega^{-1}\left(\hat{\theta}_{j n}-\theta_{j}\right),
$$

where $\sigma_{i j}$ is the $i j^{\text {th }}$ element of covariance matrix $\boldsymbol{\Sigma}$ and by setting

$$
\hat{\theta}_{0 n}=\dot{\boldsymbol{\beta}}_{0 n}(\lambda), \quad \theta_{0}=\beta_{0} 1_{k}+\sigma u, \quad \hat{\theta}_{j n}=\hat{\boldsymbol{\beta}}_{j n}(\lambda), \quad \theta_{j}=\beta, \mathbf{1}_{k},
$$

$j=1, \cdots, p$, to obtain the normal equations:

$$
\left[\begin{array}{cc}
K_{1} \Sigma & m K_{3} \\
K_{3} m^{\prime} & K_{2}
\end{array}\right]\left[\begin{array}{c}
\dot{\beta}_{n}^{*} \\
\tilde{\sigma}_{n}
\end{array}\right]=\left[\begin{array}{c}
\Sigma V \\
m^{\prime} V^{*}
\end{array}\right] .
$$

The explicit expressions for $\overline{\boldsymbol{\beta}}_{n}^{*}$ and $\tilde{\sigma}_{n}$ are given respectively by

$$
\begin{aligned}
\tilde{\beta}_{n}^{*} & =\frac{1}{\Delta}\left[K_{2} V-K_{3} V^{*}\right], \\
\text { and } \quad \tilde{\sigma}_{n} & =\frac{1}{\Delta}\left[K_{1} m^{\prime} V^{*}-K_{3} m^{\prime} V\right],
\end{aligned}
$$


where

$$
\begin{aligned}
V & =\left(V_{0}, V_{1}, \cdots, V_{p}\right)^{\prime}, \quad V_{j}=1_{k}^{\prime} \Omega^{-1} \dot{\beta}_{j n}(\lambda) ; j=0,1, \cdots, p \\
V^{*} & =\left(V_{0}^{*}, V_{1}^{*}, \cdots, V_{p}^{*}\right)^{\prime}, \quad V_{j}^{*}=u^{\prime} \Omega^{-1} \dot{\beta}_{j n}(\lambda) ; j=0,1, \cdots, p \\
K_{1} & =1_{k}^{\prime} \Omega^{-1} 1_{k}, \quad K_{2}=u^{\prime} \Omega^{-1} u, \quad K_{3}=1_{k}^{\prime} \Omega^{-1} u, \\
\Delta & =K_{1} K_{2}-K_{3}^{2}, \quad m=\left(1, \bar{x}_{1}, \ldots, \bar{x}_{p}\right)^{\prime} .
\end{aligned}
$$

The asymptotic covariance matrix of $\left(\tilde{\beta}_{n}^{* \prime}, \tilde{\sigma}_{n}\right)^{\prime}$ is given by

$$
\frac{\sigma^{2}}{n \Delta}\left[\begin{array}{cc}
K_{2} \Sigma^{-1} & -K_{3} \Sigma^{-1} m \\
-K_{3} m^{\prime} \Sigma^{-1} & K_{1}
\end{array}\right]
$$

Also, the explicit forms of $K_{1}, K_{2}$ and $K_{3}^{\prime}$ are given by

$$
\begin{aligned}
& K_{1}=\sum_{i=1}^{k+1} \frac{\left[g_{0}\left(\lambda_{i}\right)-q_{0}\left(\lambda_{1-1}\right)\right]^{2}}{\lambda_{i}-\lambda_{i-1}} \\
& K_{2}=\sum_{i=1}^{k+1} \frac{\left[g_{0}\left(\lambda_{i}\right) Q_{0}\left(\lambda_{1}\right)-q_{0}\left(\lambda_{i-1}\right) Q_{0}\left(\lambda_{i-1}\right)\right]^{2}}{\lambda_{i}-\lambda_{i-1}}, \\
& K_{3}=\sum_{i=1}^{k+1} \frac{\left[g_{0}\left(\lambda_{i}\right)-q_{0}\left(\lambda_{i-1}\right)\right]\left[g_{0}\left(\lambda_{i}\right) Q_{0}\left(\lambda_{i}\right)-q_{0}\left(\lambda_{1-1}\right) Q_{0}\left(\lambda_{i-1}\right)\right]}{\lambda_{i}-\lambda_{i-1}}
\end{aligned}
$$

with $\lambda_{0}=0$ and $\lambda_{k+1}=1$. If $F_{0}$ is symmetric and $\lambda_{i}=\lambda_{k-i+1}, i=1, \cdots, k$, then, $K_{3}=0$ and the ABLUE of $\beta^{*}$ and $\sigma$ simplify to

$$
\dot{\beta}_{n}^{*}=\frac{V}{K_{1}} \quad \text { and } \quad \tilde{\sigma}_{n}=\frac{m^{\prime} V^{*}}{K_{!}}
$$

with variance and covariance given by

$$
\operatorname{Var}\left(\dot{\beta}_{n}^{*}\right)=\frac{\sigma^{2}}{n K_{1}} \Sigma^{-1}, \quad \operatorname{Var}\left(\dot{\sigma}_{n}\right)=\frac{\sigma^{2}}{n K_{2}}
$$

and

$$
\operatorname{Cov}\left(\tilde{\beta}_{n}^{*}, \dot{\sigma}_{n}\right)=0
$$


Now, the joint asymptotic relative efficiency (JARE) of the ABLUE $\left(\tilde{\boldsymbol{\beta}}_{n}^{* \prime}, \tilde{\sigma}_{n}\right)^{\prime}$ relative to the MLE $\left(\check{\beta}_{n}^{* \prime}, \delta_{n}\right)^{\prime}$ may be computed as

$$
J A R E[A B L U E: M L E]=\frac{K_{1}^{p} \Delta}{I_{11}^{p}\left(I_{11} I_{22}-I_{12}^{2}\right)}
$$

and

$$
\begin{aligned}
A R E\left[\tilde{\beta}_{n}^{*}: M L E\right] & =\frac{I_{22}^{p+1} \Delta^{p+1}}{K_{2}^{p+1}\left(I_{11} I_{22}-I_{12}^{2}\right)^{p+1}} \\
\text { and } \operatorname{ARE}\left(\tilde{\sigma}_{n}: M L E\right) & =\frac{i_{11} \Delta}{K_{1}\left(I_{11} I_{22}-I_{12}^{2}\right)}
\end{aligned}
$$

where $I_{11}, I_{22}$ and $I_{12}$ are the elements of the informa tion matrix for a location and scale distribution. Further, for $K_{3}=0$, and $I_{12}=0$, we obtain (note that $I_{12}=0$ for symmetric distributicns)

$$
J A R E[A B L U E: M L E]=\frac{K_{1}^{p+1} K_{2}}{I_{11}^{p+1} I_{22}}
$$

and

$$
A R E\left[\tilde{\beta}_{n}^{*}: M L E\right]=K_{1}^{p+1} I_{11}^{-(p+1)} \text { and } A R E\left[\tilde{\sigma}_{n}: M L E\right]=K_{2} I_{22}^{-1}
$$

Another method of estimating $\left(\beta_{0}, \sigma\right)$ and $\beta$ would be to consider two marginal distributions namely, the marginal of

$$
\begin{array}{ll} 
& \text { (i) } n^{1 / 2}\left(\hat{\beta}_{0 n}(\lambda)-\beta_{0} 1_{k}-\sigma u\right)^{\prime} \\
\text { and } & \text { (ii) } n^{1 / 2}\left(\left(\hat{\beta}_{1 n}(\lambda)-\beta_{1} 1_{k}\right)^{\prime}, \cdots,\left(\hat{\beta}_{p n}(\lambda)-\beta_{p} 1_{k}\right)^{\prime}\right)^{\prime}
\end{array}
$$

The first one yields the estimators

$$
\begin{aligned}
\tilde{\tilde{\beta}}_{0 n} & =\frac{1}{\Delta}\left\{K_{2} V_{0}-K_{3} V_{0}^{*}\right\}, \\
\text { in.t } \quad \tilde{\tilde{\sigma}}_{n} & =\frac{1}{\Delta}\left\{K_{1} V_{0}^{*}-K_{3} V_{0}\right\},
\end{aligned}
$$

and the second one yields the estimators of $\beta$ as

$$
\tilde{\tilde{\beta}}_{n}=\frac{1}{K_{1}} V, \quad V=\left(V_{1}, \cdots, V_{p}\right)^{\prime} .
$$


The estimate of $\sigma$ is $\tilde{\tilde{\sigma}}_{n}$ which is non-negative but, (3.22) may not be non-negative. The ARE of these estimators relative to the corresponding marginal likelihood estimators may be determined easily.

Now, we consider the explicit expressions for the coefficients in (3.21) and (3.39). They are

$$
\begin{aligned}
& a=\frac{1}{\Delta}\left\{K_{2} \mathbf{1}_{k}^{\prime} \boldsymbol{\Omega}^{-1}-K_{3} u^{\prime} \boldsymbol{\Omega}^{-1}\right\}=\left(a_{1}, \cdots, a_{k}\right)^{\prime} \\
& b=\frac{1}{\Delta}\left\{K_{1} u^{\prime} \boldsymbol{\Omega}^{-1}-K_{3} \mathbf{1}_{k}^{\prime} \boldsymbol{\Omega}^{-1}\right\}=\left(b_{1}, \cdots, b_{k}\right)^{\prime}
\end{aligned}
$$

where, for $i=1,2, \ldots, k$,

$$
\begin{aligned}
a_{i}= & \frac{q_{0}\left(\lambda_{i}\right) K_{2}}{\Delta}\left\{\frac{q_{0}\left(\lambda_{i}\right)-q_{0}\left(\lambda_{i-1}\right)}{\lambda_{i}-\lambda_{i-1}}-\frac{q_{0}\left(\lambda_{i+1}\right)-q_{0}\left(\lambda_{i}\right)}{\lambda_{i+1}-\lambda_{1}}\right\}- \\
& \frac{q_{0}\left(\lambda_{i}\right) K_{3}}{\Delta}\left\{\frac{q_{0}\left(\lambda_{i}\right)^{\prime} \operatorname{co}_{0}\left(\lambda_{i}\right)-q_{0}\left(\lambda_{i-1}\right) Q_{0}\left(\lambda_{i-1}\right)}{\lambda_{i}-\lambda_{i-1}}-\frac{q_{0}\left(\lambda_{i+1}\right) Q_{0}\left(\lambda_{i+1}\right)-q_{0}\left(\lambda_{i}\right) Q_{0}\left(\lambda_{i}\right)}{\lambda_{i+1}-\lambda_{i}}\right\} \\
b_{i}= & \frac{q_{0}\left(\lambda_{i}\right) K_{1}}{\Delta}\left\{\frac{q_{0}\left(\lambda_{i}\right) Q_{0}\left(\lambda_{i}\right)-q_{0}\left(\lambda_{i-1}\right) Q_{0}\left(\lambda_{i-1}\right)}{\lambda_{i}-\lambda_{i-1}}-\frac{q_{0}\left(\lambda_{i+1}\right) Q_{0}\left(\lambda_{i+1}\right)-q_{0}\left(\lambda_{i}\right) Q_{0}\left(\lambda_{i}\right)}{\lambda_{i+1}-\lambda_{i}}\right\} \\
& -\frac{q_{0}\left(\lambda_{i}\right) K_{3}}{\Delta}\left\{\frac{q_{0}\left(\lambda_{i}\right)-q_{0}\left(\lambda_{i-1}\right)}{\lambda_{i}-\lambda_{i-1}}-\frac{q_{0}\left(\lambda_{i+1}\right)-q_{0}\left(\lambda_{i}\right)}{\lambda_{i+1}-\lambda_{i}}\right\} .
\end{aligned}
$$

Using these coefficients, we may write the estimators (3.21) and (3.22) as follows:

$$
\left[\begin{array}{c}
\tilde{\beta}_{0 n} \\
\tilde{\beta}_{1 n} \\
\vdots \\
\tilde{\beta}_{p n} \\
\tilde{\sigma}_{n}
\end{array}\right]=\left[\begin{array}{c}
a_{1} \hat{\beta}_{0 n}\left(\lambda_{1}\right)+\cdots+a_{k} \hat{\beta}_{0 n}\left(\lambda_{k}\right) \\
a_{1} \hat{\beta}_{1 n}\left(\lambda_{1}\right)+\cdots+a_{k} \hat{\beta}_{1 n}\left(\lambda_{k}\right) \\
\vdots \\
a_{1} \hat{\beta}_{p n}\left(\lambda_{1}\right)+\cdots+a_{k} \hat{\beta}_{p n}\left(\lambda_{k}\right) \\
\sum_{j=0}^{p} \bar{d}_{j}\left(b_{1} \hat{\beta}_{j n}\left(\lambda_{1}\right)+\cdots+b_{k} \dot{\beta}_{j n}\left(\lambda_{k}\right)\right)
\end{array}\right], \bar{d}_{0}=1
$$

Note that the coefficients for the estimators of the components of $\beta^{*}$ are the same satisfying the condition $\sum_{i=1}^{k} a_{i}=1$ as in Theorem 4.3 of Koenker and Bassett (1978) while the coefficients of the estimator of $\sigma$ satisfying $\sum_{j=1}^{k} b_{j}=0$. It also involves the vector $m^{\prime}=\left(1, \bar{x}_{1}, \ldots, \bar{x}_{p}\right)$. Unless $m^{\prime}=(1,0,0, \ldots, 0)$ one may not be 
able to guarantee $\tilde{\sigma}_{n}>0$. This suggest the course of estimation using (3.39) and (3.40), where there no such anamoly.

In order to obtain the ABLUE of $\left(\beta^{* \prime}, \sigma\right)^{\prime}$ optimally, we maximise $K_{1}^{p} \Delta$ with respect to $\lambda_{1}, \lambda_{2}, \ldots, \lambda_{k}$ satisfying $0<\lambda_{1}<\lambda_{2}<\cdots<\lambda_{k}<1$. Once we have obtained the optimum spacing vector $\lambda^{0}=\left(\lambda_{1}^{0}, \ldots \lambda_{k}^{0}\right)^{0}$, we can determine the optimal coefficients $a^{0}=\left(a_{1}^{0}, \ldots, a_{k}^{0}\right)^{\prime}$ and $b^{0}=\left(b_{1}^{0}, \ldots, b_{k}^{0}\right)^{\prime}$. For the class of t-distribution with chosen degrees of freedom, we have tabled in Appendix A the optimum spacings, coefficients and the values of $K_{1}^{p} \Delta$ for $k=3(1) 10$ and $\nu=1(1) 3,5,10,20,500$, which covers the Cauchy distribution as well as the normal distribution. We have also considered the Logistic distribution. To illustrate the methodology, we consider the Cauchy distribution. Here, we note that

$$
\boldsymbol{\Omega}=\left(\left(\frac{\pi^{2}\left(\lambda_{i} \wedge \lambda_{j}-\lambda_{i} \lambda_{j}\right)}{\sin ^{2}\left(\pi \lambda_{i}\right) \sin ^{2}\left(\pi \lambda_{i}\right)}\right)\right)
$$

and

$$
Q_{0}\left(\lambda_{j}\right)=\tan \left[\pi\left(\lambda_{j}-1 / 2\right)\right] ; j=1, \ldots k .
$$

The explicit forms of $K_{1}, K_{2}$ and $K_{3}$ are

$$
\begin{aligned}
& K_{1}=\sum_{i=1}^{k+1} \frac{\left[\sin ^{2}\left(\pi \lambda_{i}\right)-\sin ^{2}\left(\pi \lambda_{i-1}\right)\right]^{2}}{\pi^{2}\left(\lambda_{i}-\lambda_{i-1}\right)} \\
& K_{2}=\sum_{i=1}^{k+1} \frac{\left[\sin \left(2 \pi \lambda_{i}\right)-\sin \left(2 \pi \lambda_{i-1}\right)\right]^{2}}{\pi^{2}\left(\lambda_{i}-\lambda_{i-1}\right)} \\
& K_{3}=\sum_{i=1}^{k+1} \frac{\left[\sin ^{2}\left(\pi \lambda_{i}\right)-\sin ^{2}\left(\pi \lambda_{i-1}\right)\right]\left[\sin \left(2 \pi \lambda_{i}\right)-\sin \left(2 \pi \lambda_{i-1}\right)\right]}{2 \pi\left(\lambda_{i}-\lambda_{i-1}\right)} .
\end{aligned}
$$

The optimum spacings for the estimation of $\left(\beta^{* \prime}, \sigma\right)^{\prime}$ may be obtained by maximising $2^{p+2} K_{1} \Delta$ in this case since $I_{11}=I_{22}=1 / 2$ and $I_{12}=0$. We know that optimum spacings for the location-scale parameters are given by

$$
\lambda^{0}=\left(\frac{1}{k+1}, \frac{2}{k+1}, \cdots, \frac{k}{k+1}\right)
$$


[see for example, Balmer et al (1974) and Cane (1974)]. Using these spacings we obtain the optimum coefficients as

$$
\begin{aligned}
a_{i} & =-\frac{4}{k+1} \sin ^{4} \frac{\pi i}{k+1} \cos \frac{2 \pi i}{k+1}, i=1, \ldots, k \\
\text { and } \quad b_{i} & =-\frac{4}{k+1} \sin ^{2} \frac{\pi i}{k+1} \cos \frac{2 \pi i}{k+1}, i=1, \ldots, k
\end{aligned}
$$

These coefficients may be used to obtain the ABLUE of $\beta^{*}$ and $\sigma$ respectively. The JARE of the estimator for this case will be $\left(\frac{k+1}{\pi} \sin \frac{\pi}{k+1}\right)^{2 p+4}$.

Now, we present some numerical examples using the computational algorithms of Koenker and d'Orey $(1987,1994)$ for computing the RQs' for two sets of data.

Example 3.3.1 Consider the simple regression model $y=\beta_{0}+\beta_{1} x+z$, with the followiny data:

$$
\begin{array}{rrrrrrrrrrrrrrr}
X: & 78 & 78 & 78 & 78 & 78 & 77 & 76 & 76 & 76 & 76 & 76 & 75 & 77 & 76 \\
& 75 & 73 & 71 & 70 & 68 & 68 & 66 & 66 & 65 & 63 & 62 & 60 & 60 & \\
Y: & 17.3 & 17.6 & 15.0 & 18.1 & 18.7 & 17.9 & 18.4 & 18.1 & 16.3 & 19.4 & 17.6 & 19.5 & 12.7 & 17.0 \\
& 16.1 & 17.3 & 18.4 & 17.3 & 16.1 & 15.9 & 14.6 & 16.5 & 15.8 & 19.5 & 15.3 & 17.4 & 17.6 &
\end{array}
$$

Then, the different fitted lines are as follows,

$$
\text { LSE : } \quad 14.72224+0.0329 x
$$

Regression Median (RQ(.5)) : $\quad 9.9999+0.1 x$

Regression 1* Quantile (RQ(.25)): $\quad 9.1+0.099999 x$

Regression $3^{\text {rd }}$ Quantile $(R Q(.75)): 15.72499+0.03125 x$

Now, we represent the solutions for $k=7$ optimum regression quantiles for the 
normal distribution. They are

\begin{tabular}{c|ccc} 
& $\lambda$ & $\hat{\beta}_{0}$ & $\hat{\beta}_{1}$ \\
\hline & & & \\
1 & .0171 & 26.0000019 & -0.1727273 \\
2 & .0857 & 16.46249771 & -0.01874998 \\
3 & .2411 & 12.499999 & 0.0499999 \\
4 & .50 & 9.9999294 & 0.1000009 \\
5 & .7589 & 14.599999 & 0.049999 \\
6 & .9143 & 19.984619141 & -0.00769 \\
7 & .9829 & 19.49999 & 0.000000089
\end{tabular}

Based on these regression quantiles, we obtain the estimates of $\beta_{0}$ and $\beta_{1}$ as (14.0088, 0.04139). The following figure shows the scatter plot of data with different fitted lines. 


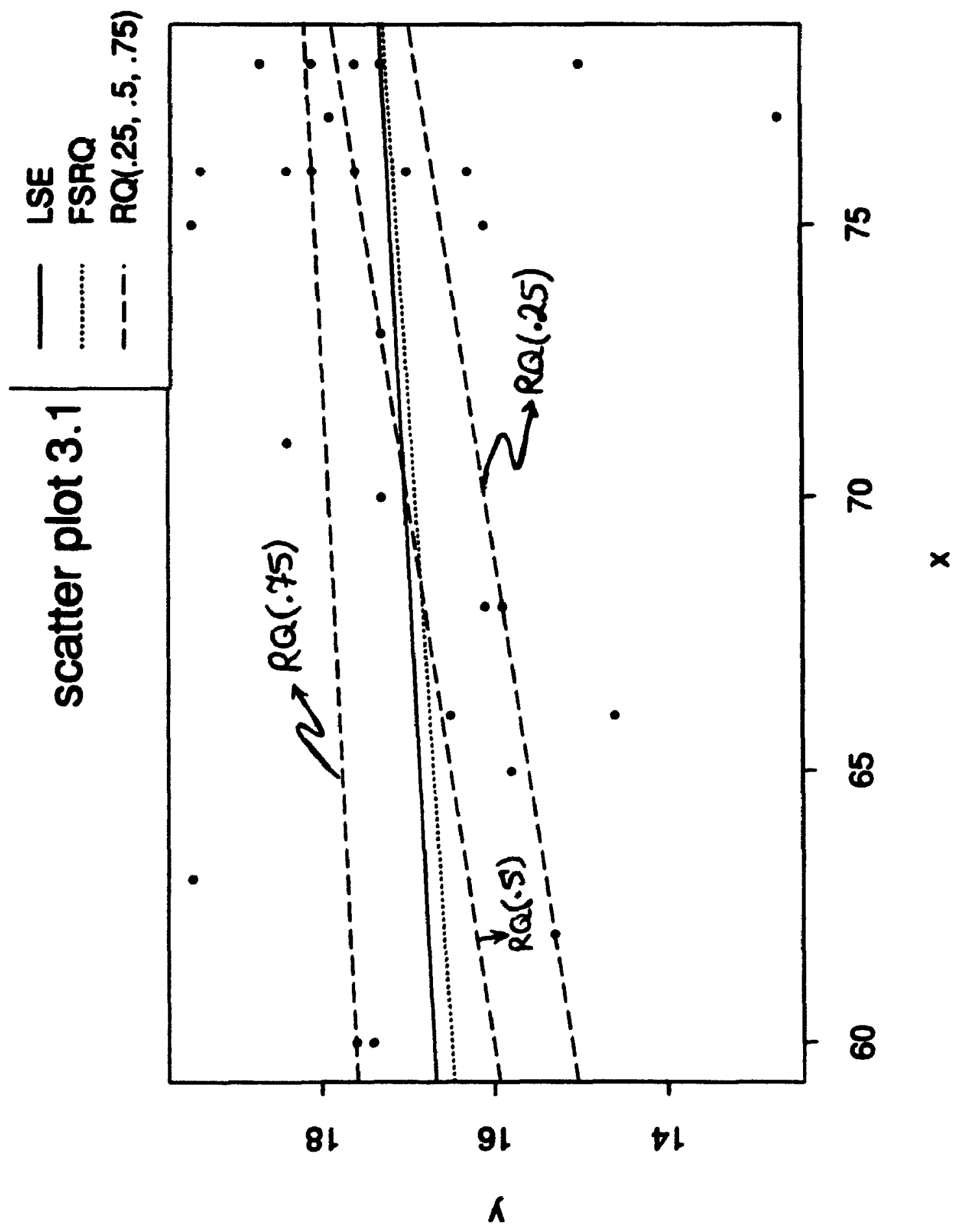


Example 3.3.2 In this example we consider the regression model

$$
y=\beta_{0}+\beta_{1} x_{1}+\beta_{2} x_{2}+\beta_{3} x_{3}+z
$$

with the following data,

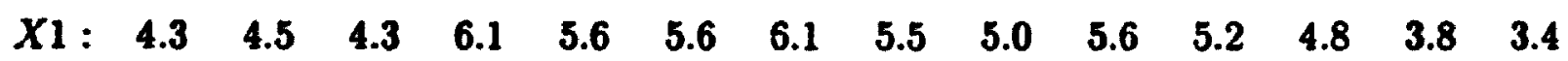

$\begin{array}{lllllllllllll}3.6 & 3.9 & 5.1 & 5.9 & 4.9 & 4.6 & 4.8 & 4.9 & 5.1 & 5.4 & 6.5 & 6.8 & 6.2\end{array}$

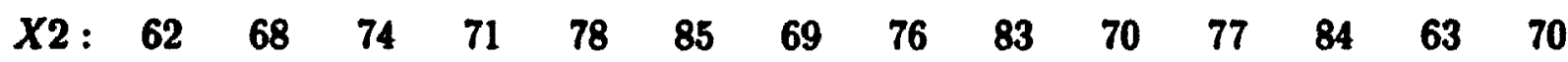

$\begin{array}{lllllllllllll}77 & 63 & 70 & 77 & 63 & 70 & 77 & 56 & 63 & 70 & 49 & 56 & 63\end{array}$

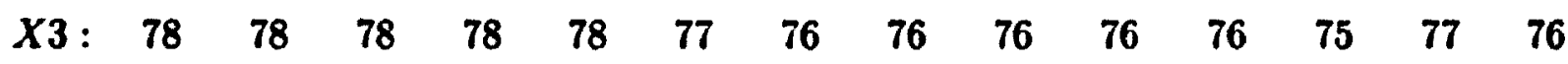

$\begin{array}{lllllllllllll}75 & 73 & 71 & 70 & 68 & 68 & 66 & 66 & 65 & 63 & 62 & 60 & 60\end{array}$

$\begin{array}{lllllllllllllll}Y: & 17.3 & 17.6 & 15.0 & 18.1 & 18.7 & 17.9 & 18.4 & 18.1 & 16.3 & 19.4 & 17.6 & 19.5 & 12.7 & 17.0\end{array}$

$\begin{array}{lllllllllllll}16.1 & 17.3 & 18.4 & 17.3 & 16.1 & 15.9 & 14.6 & 16.5 & 15.8 & 19.5 & 15.3 & 17.4 & 17.6\end{array}$

Then, the LSE fit and Regression Median fit is given,

$L S E: \quad 5.344783+0.9234785 x_{1}+0.04712587 x_{2}+0.05217684 x_{3}$

$R M: \quad 5.48703623+0.84914058 x_{1}-0.01677862 x_{2}+0.11797349 x_{3}$

In this case we obtain the solutions for $k=7$ optimum regression quantiles for the normal distribution. The results are:

\begin{tabular}{c|ccccc} 
& $\lambda$ & $\hat{\beta}_{0}$ & $\hat{\beta}_{1}$ & $\hat{\beta}_{2}$ & $\hat{\beta}_{3}$ \\
\hline & & & & & \\
1 & 0.0211 & -5.79232454 & 1.84005880 & 0.07403532 & 0.08877762 \\
2 & 0.1009 & -7.45990467 & 1.93643999 & 0.07792763 & 0.10249341 \\
3 & 0.2642 & 3.67410445 & 0.86474842 & 0.02345317 & 0.09597122 \\
4 & 0.5000 & 5.48703623 & 0.84914058 & -0.01677862 & 0.11797349 \\
5 & 0.7358 & 9.57583427 & 0.45074245 & 0.05087731 & 0.03373820 \\
6 & 0.8991 & 12.98698711 & 0.79262042 & 0.07539532 & -0.04833032 \\
7 & 0.9789 & 11.04361057 & 1.01503742 & 0.06348020 & -0.02330833
\end{tabular}


The estimates of $\beta_{0}, \beta_{1}, \beta_{2}$, and $\beta_{3}$ based on these regression quantiles are given respectively by $(5.065928,0.941837,0.0356317,0.0665379)$.

We provide here a sample table of optimum spacings, coefficients and related quantiles for ARE computation where error distribution of the linear model $(p=1)$ is Cauchy.

\begin{tabular}{|c|c|c|c|c|c|c|c|c|}
\hline$k$ & 3 & 4 & $\mathbf{s}$ & 6 & 7 & 8 & 9 & 10 \\
\hline $\begin{array}{l}\lambda_{2} \\
a_{1}\end{array}$ & $\begin{array}{l}0.2500 \\
0.0000\end{array}$ & $\begin{array}{l}0.1837 \\
-.0769\end{array}$ & $\begin{array}{l}0.1535 \\
-.0730\end{array}$ & $\begin{array}{r}0.1321 \\
-.0593\end{array}$ & $\begin{array}{l}0.1147 \\
-.0446\end{array}$ & $\begin{array}{r}0.1013 \\
-.0333\end{array}$ & $\begin{array}{l}0.0911 \\
-.0254\end{array}$ & $\begin{array}{l}0.0827 \\
-.0197\end{array}$ \\
\hline $\begin{array}{l}\lambda_{2} \\
a_{2}\end{array}$ & $\begin{array}{l}0.5000 \\
1.0000\end{array}$ & $\begin{array}{l}0.4009 \\
0.5769\end{array}$ & $\begin{array}{l}0.3465 \\
0.2664\end{array}$ & $\begin{array}{l}0.2941 \\
0.0092\end{array}$ & $\begin{array}{l}0.2500 \\
0.0000\end{array}$ & $\begin{array}{r}0.2170 \\
-.0350\end{array}$ & $\begin{array}{r}0.1926 \\
-.0453\end{array}$ & $\begin{array}{r}0.1732 \\
-.0454\end{array}$ \\
\hline $\begin{array}{l}\lambda_{3} \\
a_{3}\end{array}$ & $\begin{array}{l}0.7300 \\
0.0000\end{array}$ & $\begin{array}{l}0.5901 \\
0.5769\end{array}$ & $\begin{array}{l}0.5000 \\
0.6132\end{array}$ & $\begin{array}{l}0.4364 \\
0.4701\end{array}$ & $\begin{array}{l}0.3853 \\
0.3147\end{array}$ & $\begin{array}{l}0.3431 \\
0.1860\end{array}$ & $\begin{array}{l}0.3074 \\
0.0948\end{array}$ & $\begin{array}{l}0.2765 \\
0.0354\end{array}$ \\
\hline $\begin{array}{l}\lambda_{1} \\
a_{4}\end{array}$ & & $\begin{array}{r}0.8163 \\
-.0769\end{array}$ & $\begin{array}{l}0.6535 \\
0.2664\end{array}$ & $\begin{array}{l}0.5636 \\
0.4701\end{array}$ & $\begin{array}{l}0.50000 \\
0.4599\end{array}$ & $\begin{array}{l}0.4501 \\
0.3823\end{array}$ & $\begin{array}{l}0.1089 \\
0.2234\end{array}$ & $\begin{array}{l}0.3736 \\
0.2117\end{array}$ \\
\hline$\lambda_{s}$ & & & $\begin{array}{l}0.8465 \\
-.0730\end{array}$ & $\begin{array}{l}0.7059 \\
0.0892\end{array}$ & $\begin{array}{l}0.6147 \\
0.3147\end{array}$ & $\begin{array}{l}0.3499 \\
0.3823\end{array}$ & $\begin{array}{l}0.5000 \\
0.3650\end{array}$ & $\begin{array}{l}0.4591 \\
0.3180\end{array}$ \\
\hline $\begin{array}{l}\lambda_{6} \\
a_{6}\end{array}$ & & & & $\begin{array}{r}0.8679 \\
-.0593\end{array}$ & $\begin{array}{l}0.7500 \\
0.0000\end{array}$ & $\begin{array}{l}0.6569 \\
0.1860\end{array}$ & $\begin{array}{l}0.5911 \\
0.2934\end{array}$ & $\begin{array}{l}0.5409 \\
0.3180\end{array}$ \\
\hline $\begin{array}{l}\lambda_{7} \\
a_{7}\end{array}$ & & & & & $\begin{array}{r}0.8853 \\
-.0446\end{array}$ & $\begin{array}{r}0.7830 \\
-.0350\end{array}$ & $\begin{array}{l}0.6926 \\
0.0048\end{array}$ & $\begin{array}{l}0.6264 \\
0.2117\end{array}$ \\
\hline$\lambda_{0}$ & & & & & & $\begin{array}{l}0.8987 \\
-.0333\end{array}$ & $\begin{array}{l}0.8074 \\
-.0453\end{array}$ & $\begin{array}{l}0.7235 \\
0.0354\end{array}$ \\
\hline $\begin{array}{l}\lambda_{0} \\
a_{0}\end{array}$ & & & & & & & $\begin{array}{l}0.9089 \\
-.0254\end{array}$ & $\begin{array}{r}0.8268 \\
-.0454\end{array}$ \\
\hline $\begin{array}{l}\lambda_{10} \\
a_{10}\end{array}$ & & & & & & & & $\begin{array}{r}0.9173 \\
-.0197\end{array}$ \\
\hline $\begin{array}{l}K_{1} \\
K_{2} \\
K_{3} \\
\Delta\end{array}$ & $\begin{array}{l}0.4053 \\
0.4053 \\
0.0000 \\
0.0666\end{array}$ & $\begin{array}{l}0.4469 \\
0.4244 \\
0.0000 \\
0.0848\end{array}$ & $\begin{array}{l}0.4631 \\
0.4457 \\
0.0000 \\
0.0956\end{array}$ & $\begin{array}{l}0.4712 \\
0.4619 \\
0.0000 \\
0.1025\end{array}$ & $\begin{array}{l}0.4774 \\
0.4713 \\
0.0000 \\
0.1074\end{array}$ & $\begin{array}{l}0.4822 \\
0.4770 \\
0.0000 \\
0.1109\end{array}$ & $\begin{array}{l}0.4855 \\
0.4813 \\
0.0000 \\
0.1135\end{array}$ & $\begin{array}{l}0.4880 \\
0.4846 \\
0.0000 \\
0.1154\end{array}$ \\
\hline
\end{tabular}

In this case, as an example, if we choose $k=7$ the ARE is

$$
A R E=K_{1} I_{11}^{-1}=(0.4774) \times 2=0.9548
$$

\subsubsection{Estimation of Conditional Quantile Function}

In this subsection, we consider the estimation of a conditional quantile function defined by

$$
Q(\lambda)=t_{0}^{\prime} \beta^{*}+\sigma Q_{0}(\lambda), \quad 0<\lambda<1
$$


based on a few, say $k,(p+2 \leq k<n)$ selected RQ's where $t_{0}$ is a prefixed vector in advance. To achieve this, we simply substitute the ABLUE of $\left(\beta^{* \prime}, \sigma\right)^{\prime}$ in (3.51) from (3.21) and (3.22) and obtain

$$
\tilde{Q}_{n}(\lambda)=t_{0}^{\prime} \tilde{\beta}_{n}^{*}+\tilde{\sigma}_{n} Q_{0}(\lambda), \quad \text { for each } \lambda \in(0,1) .
$$

Then the asymptotic variance is given by

$$
\operatorname{Var}\left(\tilde{Q}_{n}(\lambda)\right)=\frac{\sigma^{2}}{n \Delta}\left\{K_{2} t_{0}^{\prime} \Sigma^{-1} t_{0}-2 K_{3} t_{0}^{\prime} \Sigma^{-1} m Q_{0}(\lambda)+K_{1} Q_{0}^{2}(\lambda)\right\}
$$

Similarly, the MLE of $Q(\lambda)$, say, $Q_{n}(\lambda)$ has the asymptotic variance given by

$$
\frac{\sigma^{2}}{n|I|}\left\{I_{22} t_{0}^{\prime} \Sigma^{-1} t_{0}-2 I_{12} t_{0}^{\prime} \Sigma^{-1} m Q_{0}(\lambda)+I_{11} Q_{0}^{2}(\lambda)\right\}
$$

The ARE of $\tilde{Q}_{n}(\lambda)$ relative to $Q_{n}(\lambda)$ is given by (3.54) divided by (3.53). The ARE expression lies between

$$
C h_{\min }\left(K^{*} I^{--1}\right) \leq A R E\left[\tilde{Q}_{n}(\lambda): \bar{Q}_{n}(\lambda)\right] \leq C h_{\max }\left(K^{*} I^{*-1}\right)
$$

where

$$
K^{*}=\left(\begin{array}{cc}
K_{1} \Sigma & m K_{3} \\
K_{3} m^{\prime} & K_{2}
\end{array}\right) \text { and } I^{*}=\left(\begin{array}{cc}
I_{11} \Sigma & m I_{12} \\
I_{12} m^{\prime} & I_{22}
\end{array}\right) \text {, }
$$

and $C h_{\min }(A)$ and $C h_{\max }(A)$ are a minimum and maximum characteristic root of $A$. Now, consider two quantities namely $(i) \operatorname{tr}\left[K^{*} I^{*-1}\right]$ and $(i i)\left|K^{*} I^{*-1}\right|^{1 / p+2}$. Then, $\frac{1}{p+2} \operatorname{tr}\left[K^{*} I^{*-1}\right]$ will provide us with the average ARE of $\tilde{Q}_{n}(\lambda)$ relative to $\bar{Q}_{n}(\lambda)$ which lies between $C h_{\min }\left(K^{*} I^{*-1}\right)$ and $C h_{\max }\left(K^{*} I^{*-1}\right)$ while $\left|K^{*} I^{*-1}\right|^{1 / p+2}$ gives the geometric mean of the characteristics roots of $K^{*} I^{*-1}$. Hence, we determine the average $A R E$ as

$$
\frac{1}{p+2} \operatorname{tr}\left[K^{*} I^{*-1}\right]=\left[(p+1) K_{1} I_{22}-2 K_{3} I_{12}+K_{2} I_{11}\right] /(p+2) .
$$

On the other hand,

$$
\left|K^{*} I^{*-1}\right|=\frac{K_{1}^{p} \Delta}{I_{11}^{p}\left(I_{11} I_{22}-I_{12}^{2}\right)}
$$


The optimization of (3.57) or (3.58) will provide the required optimum spacings for the determination of the regression quantiles required to estimate $\left(\beta^{* \prime}, \sigma\right)^{\prime}$ and thereby $Q(\lambda)$. It is clear that (3.57) and (3.58) do not depend on the design matrix $D_{n}$. Alternatively, if $f_{0}(z)$ is symmetric then, ihe $(p+2)$ characteristic roots of $\left(K^{*} I^{*-1}\right)$ are

$$
\begin{aligned}
& K_{1} I_{11}^{-1}, \ldots, K_{1} I_{11}^{-1}, K_{1} I_{11}^{-1}, \\
& \quad\left(K_{1} I_{11}^{-1}+K_{2} I_{22}^{-1}\right) / 2 \pm 1 / 2\left(\left(K_{1} I_{11}^{-1}-K_{2} I_{22}^{-1}\right)^{2}+4 K_{3} I_{11}^{-1} I_{22}^{-1}\right)^{1 / 2}
\end{aligned}
$$

Thus, we maximise

$$
\left(K_{1} I_{11}^{-1}+K_{2} I_{22}^{-1}\right) / 2+1 / 2\left(\left(K_{1} I_{11}^{-1}-K_{2}^{\prime} I_{22}^{-1}\right)^{2}+4 K_{3} I_{11}^{-1} I_{22}^{-1}\right)^{1 / 2}
$$

w.r.t $Q_{0}(\lambda)$ to obtain the optimum spacing vector which is independent of $D_{n}$.

\subsubsection{Estimating functions of parameters}

Let $g\left(\beta^{*}, \sigma\right)$ be a function of the regression and scale parameters of the linear model. We consider the estimation of $g\left(\beta^{*}, \sigma\right)$ based on $k,(p+2 \leq k \leq n)$ regression quantiles $\hat{\boldsymbol{\beta}}_{j n}(\lambda)=\left(\hat{\beta}_{j n}\left(\lambda_{1}\right), \ldots, \hat{\beta}_{j n}\left(\lambda_{k}\right)\right)^{\prime}, j=0,1, \ldots, p$ as in $(3.18)$ and then estimate $g\left(\beta^{*}, \sigma\right)$ by the substitution method as $g\left(\check{\beta}^{*}, \tilde{\sigma}\right)$. To study the properties of this estimator, we use the local linearization theorem as in Rao (1973). This allows us to obtain the asymptotic variance of $g\left(\tilde{\beta}^{*}, \tilde{\sigma}\right)$.

Now, assume that $g\left(\beta^{*}, \sigma\right)$ is defined on $\mathbb{R}^{p+1} \times \mathbb{R}^{+}$possessing continuous partial derivatives of order at least two at each point of $\mathbb{R}^{p+1} \times \mathbb{R}^{+}$. Then, using Rao (1973), the asymptotic variance of $g\left(\check{\beta}^{*}, \tilde{\sigma}\right)$ may be written as

$$
\operatorname{Var}\left(g\left(\tilde{\beta}^{*}, \tilde{\sigma}\right)\right)=\frac{\sigma^{2}}{n} \omega^{\theta} K^{*-1} \omega, \omega=\left(\omega_{0}, \omega_{1}, \ldots, \omega_{p+1}\right)^{\prime},
$$

where

$$
\omega_{i}=\frac{\partial g\left(\beta^{*}, \sigma\right)}{\partial \beta_{i}}, i=0,1, \ldots, p ; \omega_{p+1}=\frac{\partial g\left(\beta^{*}, \sigma\right)}{\partial \sigma}
$$


and $\mathrm{K}^{*-1}$ is the covariance factor in section 3.3.1.

Similarly, let $g\left(\bar{\beta}^{*}, \sigma\right)$ be the MLE of $g\left(\beta^{*}, \sigma\right)$ with asymptotic variance

$$
\operatorname{Var}\left[g\left(\bar{\beta}^{*}, \delta\right)\right]=\frac{\sigma^{2}}{n} \omega^{\prime} \Gamma^{*-1} \omega .
$$

The ARE of $g\left(\overline{\boldsymbol{\beta}}^{*}, \tilde{\sigma}\right)$ relative to $g\left(\overline{\boldsymbol{\beta}}^{*}, \bar{\sigma}\right)$ is given by

$$
A R E\left[g\left(\tilde{\beta}^{*}, \tilde{\sigma}\right): g\left(\bar{\beta}^{*}, \tilde{\sigma}\right)\right]=\frac{\omega^{\prime} \boldsymbol{I}^{*-1} \omega}{\omega^{\prime} \boldsymbol{K}^{*-1} \omega} .
$$

To obtain the optimum spacing vector for the estimation of $g\left(\beta^{*}, \sigma\right)$, one has to maximize (3.63) with respect to $Q_{0}\left(\lambda_{1}\right), \ldots, Q_{0}\left(\lambda_{k}\right)$ for fixed vector $\omega$. However, the function $g\left(\beta^{*}, \sigma\right)$ is nonlinear in general and the vector $\omega$ depends on the parameters $\left(\beta^{* \prime}, \sigma\right)^{\prime}$ which is unknown, therefore, we may apply the Courant-Fisher Theorem (Rao, 1973) and find that

$$
C h_{\min }\left(K^{*} \Gamma^{*-1}\right) \leq A R E \leq C h_{\max }\left(K^{*} \Gamma^{*-1}\right) .
$$

Now, we follow section 3.3.2, for optimization problem to determine the optimum spacings. In this way one is able to estimate functions of $\left(\beta^{\prime \prime}, \sigma\right)^{\prime}$ such as, (i) conditional survival function and (ii) conditional hazard function .

\subsection{Trimmed Least-Squared Estimation of Re- gression Parameters And Its Asymptotic Dis- tribution}

In the previous section we considered the L-estimation of regression and scale parameters based on a few selected regression quantiles. These estimators resulted in quick and robust estimator of $\beta$ and $\sigma$ against outliers for each distribution under consideration while sacrificing very little on the asymptotic relative efficiency. The basic idea of derivation was based on thr: generalized least squares principle. In 
this section we are going to consider another L-estimator namely, the trimmed least squares estimator (TLSE) of $\beta$.

Consider the regression model

$$
Y=D_{n} \beta+Z, \text { where } \beta=\left(\beta_{0}, \beta_{1}, \ldots, \beta_{p}\right)^{\prime} \in \mathbb{R}^{p+1}
$$

where $Z=\left(Z_{1}, \cdots, Z_{n}\right)^{\prime}$ has components which are i.i.d r.v's from a continuous $c d f$ $F_{0}(z)$ (with $p d f f_{0}(z)$ ). The least squares estimator (LSE) of $\beta$ is given by

$$
\tilde{\beta}_{n}^{L}=\left(D_{n}^{\prime} D_{n}\right)^{-1} D_{n}^{\prime} Y
$$

which is obtained by minimizing

$$
\left(\boldsymbol{Y}-D_{n} \beta\right)^{\prime}\left(\boldsymbol{Y}-D_{n} \beta\right)
$$

with respect to $\beta$. For the trimmed least squares estimation (TLSE) of $\beta$, we first define an $n \times n$ diagonal matrix $A$ whose elements are

$$
a_{i i}=\left\{\begin{array}{cc}
0, & \text { if } Y_{i} \leq \alpha_{i} \hat{\beta}_{n}\left(\lambda_{1}\right) \text { or } Y_{i}>\alpha_{i} \hat{\beta}_{n}\left(\lambda_{2}\right) \\
1, & \text { otherwise }
\end{array}\right.
$$

where $\hat{\beta}_{n}\left(\lambda_{1}\right)$ and $\hat{\beta}_{n}\left(\lambda_{2}\right)$ are the regression quantiles of orders $\lambda_{1}$ and $\lambda_{2}$ respectively for $0<\lambda_{1}<1 / 2<\lambda_{2}<1$. We obtain the TLSE of $\beta$ by minimising

$$
\left(\boldsymbol{Y}-D_{n} \beta\right)^{\prime} A\left(Y-D_{n} \beta\right)
$$

with respect to $\boldsymbol{\beta}$ obtaining

$$
\bar{\beta}_{n}\left(\lambda_{1}, \lambda_{2}\right)=\left(D_{n}^{\prime} A D_{n}\right)^{-1} D_{n}^{\prime} A Y .
$$

Ruppert and Carroll (1980) studied the TLSE under the conditions

1. $F_{0}$ has continuous density $f_{0}$ that is positive on the support of $F_{0}$.

2. The first column of design matrix is 1 , i.e $d_{12}=1 i=1, \cdots, n$ and

$$
\sum_{i=1}^{n} d_{i j}=0, \text { for } j=2, \cdots, p+1 \text {. }
$$


3. $\quad \lim _{n \rightarrow \infty}\left(\max _{i j}\left(n^{-1 / 2}\left|d_{i j}\right|\right)\right)=0$.

4. There exists positive definite $\boldsymbol{\Sigma}$ such that

$$
\lim _{n \rightarrow \infty} n^{-1}\left(D_{n}^{\prime} D_{n}\right)=\Sigma
$$

Jurecková (1984) stated the representation and asymptotic properties of $\bar{\beta}_{n}\left(\lambda_{1}, \lambda_{2}\right)$ under the following conditions:

i) $F_{0}$ is absolutely continuous with density function $f_{0}$ such that $f_{0}(\cdot)>0$ and $f_{0}^{\prime}(\cdot)$ is bounded in a neighborhood of $F_{0}^{-1}(\lambda)$.

ii) $\quad \max _{i, j} n^{-1 / 4}\left|d_{i j}\right|=O(1)$, as $n \rightarrow \infty$.

iii) $\quad \lim _{n \rightarrow \infty} n^{-1}\left(D_{n}^{\prime} D_{n}\right)=\Sigma$, where $\Sigma$ is a positive definite $(p+1) \times(p+1)$ matrix.

iv) $\quad \frac{1}{n} \sum_{i=1}^{n} d_{i j}^{n}=O(1)$, as $n \rightarrow \infty, j=1, \cdots, p+1$.

v) $\lim _{n \rightarrow \infty} \bar{d}_{n j}=\bar{d}_{j}, j=1, \cdots, p+1$.

We are going to obtain the same results as in Ruppert and Carroll (1980) and Jurexková (1984) under weaker conditions on the design matrix $D_{n}$ and distribution function $F_{0}$. For this reason we use the weighted empirical approach of Koul (1992) providing new proofs for the theorems and lemmas that follows later on. Before we state the main theorem for TSLE we need an asymptotic linearity result for $\dot{\boldsymbol{\beta}}_{n}(\lambda)$. First, we consider the conditions A0, A1 and A2 from section 3.2 and define

$$
\beta(\lambda)=\beta+Q_{0}(\lambda) e_{1}, \quad e_{1}=(1,0, \cdots, 0)
$$

Now, consider the weighted empirical process

$$
T_{n}(t ; \lambda)=n^{-1 / 2} \sum_{i=1}^{n} d_{i}\left\{I\left(y_{i}-d_{i}^{d} t \leq 0\right)-\lambda\right\}
$$

which is basic for Theorem 3.4.1 given below.

Theorem 3.4.1 Under the conditions $A 0, A 1$ and $A 2$ for each $\lambda \in(0,1)$ we have

$$
n^{1 / 2}\left(\dot{\beta}_{n}(\lambda)-\beta(\lambda)\right)=-\left\{q_{0}(\lambda) \Sigma_{n}\right\}^{-1} T_{n}(\beta(\lambda) ; \lambda)+o_{P}(1)
$$


where

$$
T_{n}(\beta(\lambda) ; \lambda)=n^{-1 / 2} \sum_{i=1}^{n} d_{i}\left\{I\left(Z_{i} \leq Q_{0}(\lambda)\right)-\lambda\right\}
$$

We prove this Theorem with the aid of two Lemmas.

Lemma 3.4.2 Let the conditions of Theorem 3.4.1 hold. Then for each $0<\lambda<$ 1 and $6>0$

$$
\sup _{\|t\| \leq b}\left\|T_{n}\left(\beta(\lambda)+n^{-1 / 2} t ; \lambda\right)-T_{n}(\beta(\lambda) ; \lambda)-q_{0}(\lambda) \Sigma_{n} t\right\|=o P(1)
$$

Proof:

This lemma is a weaker version of Theorem 2.3.1 of Koul (1992) where he proves the above result uniformly in $\lambda$ when $\mathrm{AO}$ is replaced by assuming $f_{0}$ to be uniformly continuous. Denote by $T_{n}(t ; \lambda)=\left(T_{n 0}(t ; \lambda) . \cdots, T_{n p}(t ; \lambda)\right)$. So, we prove that

$$
\sup _{\|t\| \leq b}\left|T_{n j}\left(\beta(\lambda)+n^{-1 / 2} t ; \lambda\right)-T_{n j}(\beta(\lambda) ; \lambda)-g_{0}(\lambda) \frac{1}{n} \sum_{i=1}^{n} d_{i j} d_{i}^{\prime} t\right|=o_{P}(1) \text {. }
$$

by considering the centered $T_{n}\left(\beta(\lambda)+n^{-1 / 2} t ; \lambda\right)$

$$
T_{n j}^{0}(t ; \lambda)=T_{n j}\left(\beta(\lambda)+n^{-1 / 2} t ; \lambda\right)-E\left[T_{n j}\left(\beta(\lambda)+n^{-1 / 2} t ; \lambda\right)\right]
$$

and the compact set

$$
\mathcal{N}(b)=\left\{t \in \mathbb{R}^{p+1} \mid\|t\| \leq b\right\}, 0<b<\infty
$$

Then, the expression (3.76) is bounded above by

$$
\begin{aligned}
& \sup _{\|t\| \leq b}\left|T_{n j}^{0}(t ; \lambda)-T_{n j}^{0}(0 ; \lambda)\right| \\
& \quad+\sup _{\|t\| \leq b}\left|E\left[T_{n j}\left(\beta(\lambda)+n^{-1 / 2} t ; \lambda\right)\right]-E\left[T_{n j}(\beta(\lambda) ; \lambda)\right]-g_{0}(\lambda) \frac{1}{n} \sum_{i=1}^{n} d_{i j} d_{i}^{\prime} t\right| \\
& =I_{1}+I_{2} .
\end{aligned}
$$

First, we consider $I_{2}$ where

$$
I_{2}=\sup _{\|:\| \leq b}\left|n^{-1 / 2} \sum_{i=1}^{n} d_{i j}\left\{F_{0}\left(C_{\nu}(\lambda)+n^{-1 / 2} d_{i}^{\prime} t\right)-F_{0}\left(Q_{0}(\lambda)\right)-y_{0}(\lambda) n^{-1 / 2} d_{i}^{*} t\right\}\right|
$$




$$
\begin{gathered}
\leq n^{-1 / 2} \sum_{i=1}^{n}\left|d_{i j}\right| \sup _{\|t\| \leq b} \mid F_{0}\left(Q_{c}(\lambda)+n^{-1 / 2} d_{i}^{\prime} t\right)-F_{0}\left(Q_{0}(\lambda)\right) \\
-q_{0}(\lambda) n^{-1 / 2} d_{i}^{\prime} t \mid .
\end{gathered}
$$

By the mean value theorem $\exists \Delta_{n}(t) \in\left(0, n^{-1 / 2} d_{i}^{\prime} t\right)$ such that

$$
I_{2} \leq n^{-1 / 2} \sum_{i=1}^{n}\left|d_{i j}\right| \sup _{\|t\| \leq b}\left|n^{-1 / 2} d_{i} t\right|\left|f_{0}\left(Q_{0}(\lambda)+\Delta_{n}(t)\right)-f_{0}\left(Q_{0}(\lambda)\right)\right|
$$

and by taking $\delta_{n}=n^{-1 / 2}\left\|d_{i}\right\| b$ we have

$$
\begin{aligned}
I_{2} & \leq n^{-1} \sum_{i=1}^{n}\left|d_{i j}\right|\left\|d_{i}\right\| b \sup _{\Delta_{n}(t) \leq \delta_{n}}\left|f_{0}\left(Q_{0}(\lambda)+\Delta_{n}(t)\right)-f_{0}\left(Q_{0}(\lambda)\right)\right| \\
& =o(1) \text { as } n \rightarrow \infty \text { and conditions A0 and A2 }
\end{aligned}
$$

Now, we prove that $I_{1}=o p(1)$ where

$$
I_{1}=\sup _{\|t\| \leq b}\left|T_{n j}^{0}(t ; \lambda)-T_{n j}^{0},(0 ; \lambda)\right| .
$$

First, we prove it for each $t \in \mathcal{N}(b)$

$$
\begin{aligned}
& \left|T_{n j}^{0}(\mathbf{t} ; \lambda)-T_{n j}^{0}(0 ; \lambda)\right| \\
& =\mid n^{-1 / 2} \sum_{i=1}^{n} d_{i j}\left\{I\left(Z_{i} \leq Q_{0}(\lambda)+n^{-1 / 2} d_{i}^{\prime} \mathbf{t}\right)-I\left(Z_{i} \leq Q_{0}(\lambda)\right)\right. \\
& \left.\quad-F_{0}\left(Q_{0}(\lambda)+n^{-1 / 2} d_{i}^{\prime} \mathbf{t}\right)+F_{0}\left(Q_{0}(\lambda)\right)\right\} \mid \\
& =\left|n^{-1 / 2} \sum_{i=1}^{n} d_{i j}\left\{V_{i}(\mathbf{t})-\nu_{n i}(\mathbf{t})\right\}\right|
\end{aligned}
$$

where

$$
\begin{aligned}
V_{i}(\mathrm{t}) & =I\left(Q_{0}(\lambda)<Z_{i} \leq\left(Q_{0}(\lambda)+n^{-1 / 2} d_{i}^{\prime} t\right)\right. \\
\nu_{n i}(\mathrm{t}) & =F_{0}\left(Q_{0}(\lambda)+n^{-1 / 2} d_{i}^{\prime} t\right)-F_{0}\left(Q_{0}(\lambda)\right)
\end{aligned}
$$

Now, we apply the Chebyshev inequality to get the result.

$$
P\left(\left|n^{-1 / 2} \sum_{i=1}^{n} d_{i j}\left\{V_{i}(t)-\nu_{n i}(t)\right\}\right|^{2}>\epsilon^{2}\right)
$$




$$
\begin{aligned}
& \leq \frac{1}{\epsilon^{2}} \frac{1}{n} E\left(\sum_{i=1}^{n} d_{i j}\left\{V_{i}(t)-\nu_{n i}(t)\right\}\right)^{2} \\
& =\frac{1}{\epsilon^{2}} \frac{1}{n} \sum_{i} \sum_{k} d_{i j} d_{k j} E\left(V_{i}(t)-\nu_{n i}(t)\right)\left(V_{k}(t)-\nu_{n k}(t)\right) \\
& =\frac{1}{\epsilon^{2}} \frac{1}{n} \sum_{i=1}^{n} d_{i j}^{2} E\left(V_{i}(t)-\nu_{n i}(t)\right)^{2} \\
& \leq \epsilon \text { as } n \rightarrow \infty \text { by A0 and A2, }
\end{aligned}
$$

which implies $I_{1}=o_{P}(1)$ for fixed $t$. For tightness, we use the compactness property of set $\mathcal{N}(b)$. Since, $\mathcal{N}(b)$ is a compact set it is suffices to show that for each $s \in \mathcal{N}(b)$ and for every $\epsilon>0$, there is a $\delta>0$ such that

$$
\operatorname{limsu}_{n}, P\left(\sup _{\|t-s\| \leq \delta}\left|T_{n j}^{0}(t ; \lambda)-T_{n j}^{0}(s ; \lambda)\right|>2 \epsilon\right)<\epsilon .
$$

We need the following relations to prove (3.87)

$$
d_{i}^{\prime} \mathrm{t}=d_{i}^{\prime} s+\left(d_{i}^{\prime} \mathrm{t}-d_{i}^{\prime} s\right)
$$

So that

$$
d_{i}^{\prime} \mathrm{t}-d_{z}^{\prime} s=d_{i}^{\prime}(\mathrm{t}-s)
$$

and

$$
\begin{aligned}
\left|d_{i}^{\prime} \mathrm{t}-d_{i}^{\prime} s\right| & =\left|d_{i}^{\prime}(\mathbf{t}-s)\right| \\
& \leq\left\|d_{i}\right\|\|\mathbf{t}-s\| \\
& \leq\left\|d_{i}\right\| \delta .
\end{aligned}
$$

Thus,

$$
d_{i}^{\prime} s-\left\|d_{i}\right\| \delta \leq d_{i}^{\prime} t \leq d_{i}^{\prime} s+\left\|d_{i}\right\| \delta .
$$

Therefore, we have

$$
\left|T_{n j}^{0}(\mathbf{t} ; \lambda)-T_{n j}^{0}(s ; \lambda)\right|=
$$




$$
\begin{gathered}
\mid n^{-1 / 2} \sum_{i=1}^{n} d_{i j}\left\{\left[I\left(Z_{i} \leq Q_{0}(\lambda)+n^{-1 / 2} d_{i}^{\prime} t\right)-I\left(Z_{i} \leq Q_{0}(\lambda)+n^{-1 / 2} d_{i}^{\prime} s\right)\right]\right. \\
\left.-\left[F_{0}\left(Q_{0}(\lambda)+n^{-1 / 2} d_{i}^{\prime} t\right)-F_{0}\left(Q_{0}(\lambda)+n^{-1 / 2} d_{i}^{\prime} s\right)\right]\right\} \mid \\
\leq n^{-1 / 2} \sum_{i=1}^{n}\left|d_{i j}\right| \mid I\left(Z_{i} \leq Q_{0}(\lambda)+n^{-1 / 2} d_{i}^{\prime} t\right) \\
-I\left(Z_{i} \leq Q_{0}(\lambda)+n^{-1 / 2} d_{i}^{\prime} s\right) \mid \\
+n^{-1 / 2} \sum_{i=1}^{n}\left|d_{i j}\right|\left|F_{0}\left(Q_{0}(\lambda)+n^{-1 / 2} d_{i}^{\prime} t\right)-F_{0}\left(Q_{0}(\lambda)+n^{-1 / 2} d_{i}^{\prime} s\right)\right|
\end{gathered}
$$

Now, using the monotone property of the indicator function with the relation (3.90), we can write the first term of (3.91) under the absolute sign as

$$
\begin{aligned}
& {\left[I\left(Z_{i} \leq Q_{0}(\lambda)+n^{-1 / 2} d_{i}^{\prime} s-n^{-1 / 2}\left\|d_{i}\right\| \delta\right)-I\left(Z_{i} \leq Q_{0}(\lambda)+n^{-1 / 2} d_{i}^{\prime} s\right)\right]} \\
& \quad \leq\left[I\left(Z_{i} \leq Q_{0}(\lambda)+n^{-1 / 2} d_{i}^{\prime} t\right)-I\left(Z_{i} \leq Q_{0}(\lambda)+n^{-1 / 2} d_{i}^{\prime} s\right)\right] \\
& \leq\left[I\left(Z_{i} \leq Q_{0}(\lambda)+n^{-1 / 2} d_{i}^{\prime} s+n^{-1 / 2}\left\|d_{i}\right\| \delta\right)-I\left(Z_{i} \leq Q_{0}(\lambda)+n^{-1 / 2} d_{i}^{\prime} s\right)\right]
\end{aligned}
$$

so that

$$
\begin{aligned}
& \left|I\left(Z_{i} \leq Q_{0}(\lambda)+n^{-1 / 2} d_{i}^{\prime} \mathrm{t}\right)-I\left(Z_{i} \leq Q_{0}(\lambda)+n^{-1 / 2} d_{i}^{\prime} s\right)\right| \leq \\
& \leq \mid I\left(Z_{i} \leq Q_{0}(\lambda)+n^{-1 / 2} d_{i}^{\prime} s+n^{-1 / 2}\left\|d_{i}\right\| \delta\right)- \\
& I\left(Z_{i} \leq Q_{0}(\lambda)+n^{-1 / 2} d_{i}^{\prime} s-n^{-1 / 2}\left\|d_{i}\right\| \delta\right) \mid
\end{aligned}
$$

and for the second term, we have

$$
\begin{aligned}
& \left|F_{0}\left(Q_{0}(\lambda)+n^{-1 / 2} d_{i}^{\prime} \mathrm{t}\right)-F_{0}\left(Q_{0}(\lambda)+n^{-1 / 2} d_{i}^{\prime} s\right)\right| \\
& \quad \leq F_{0}\left(Q_{0}(\lambda)+n^{-1 / 2} d_{i}^{\prime} s+n^{-1 / 2}\left\|d_{i}\right\| \delta\right)-F_{0}\left(Q_{0}(\lambda)+n^{-1 / 2} d_{i}^{\prime} s-n^{-1 / 2}\left\|d_{i}\right\| \delta\right) .
\end{aligned}
$$

If we substitute (3.93) and (3.94) in (3.91) then add and subtract the corresponding mean, we have

$$
\left|\cdot i_{i j}^{+0}(\mathbf{t} ; \lambda)-T_{n j}^{0}(s ; \lambda)\right| \leq
$$




$$
\begin{array}{r}
n^{-1 / 2} \sum_{i=1}^{n}\left|d_{i j}\right|\left\{\mid I\left(Z_{i} \leq Q_{0}(\lambda)+n^{-1 / 2} d_{i}^{\prime} s+n^{-1 / 2}\left\|d_{i}\right\| \delta\right)\right. \\
\left.-F_{0}\left(Q_{0}(\lambda)+n^{-1 / 2} d_{i}^{\prime} s+n^{-1 / 2}\left\|d_{i}\right\| \delta\right)\right] \\
-\left[I\left(Z_{i} \leq Q_{0}(\lambda)+n^{-1 / 2} d_{i}^{\prime} s-n^{-1 / 2}\left\|d_{i}\right\| \delta\right)\right\} \\
\left.\left.-F_{0}\left(Q_{0}(\lambda)+n^{-1 / 2} d_{i}^{\prime} s-n^{-1 / 2}\left\|d_{i}\right\| \delta\right)\right]\right\} \\
+2 n^{-1 / 2} \sum_{i=1}^{n}\left|d_{i j}\right|\left\{F_{0}\left(Q_{0}(\lambda)+n^{-1 / 2} d_{i}^{\prime} s+n^{-1 / 2}\left\|d_{i}\right\| \delta\right)\right. \\
\left.-F_{0}\left(Q_{0}(\lambda)+n^{-1 / 2} d_{i}^{\prime} s-n^{-1 / 2}\left\|d_{i}\right\| \delta\right)\right\} .
\end{array}
$$

As we see, the R.H.S of (3.95) (designating it as $I_{11}$ and $I_{12}$ respectively), does not depend on $t$ anymore and $\boldsymbol{s}$ is fixed. Now we apply the same argument as we mentioned in the proof of $I_{1}$ for fixed $t$ to obtain $I_{11}=o_{P}(1)$. By condition $A 0$ and A2, $I_{12}<\epsilon$ for sufficiently small $\delta$ which completes the proof of Lemma 3.4.2.

The next Lemma will show that the regression quantiles are bounded in probability, i.e

Lemma 3.4.3 Under the condition of Theorem 3.4.1

$$
n^{1 / 2}\left(\hat{\beta}_{n}(\lambda)-\beta(\lambda)\right)=O_{P}(1) .
$$

We need the following steps to prove this Lemma

i)

$$
\begin{aligned}
T_{n j}\left(\hat{\beta}_{n}(\lambda) ; \lambda\right) & =n^{-1 / 2} \sum_{i=1}^{n} d_{\imath}\left\{I\left(y_{\imath}-d_{i}^{\prime} \hat{\beta}_{n}(\lambda) \leq 0\right)-\lambda\right\} \\
& =o_{P}(1)
\end{aligned}
$$

ii)

$$
T_{n j}(\beta(\lambda) ; \lambda)=O_{P}(1)
$$

iii) $\forall \epsilon>0,0<u<\infty \exists b\left(=b_{u}^{c}\right)$ and an $N^{\iota}$ such that for each $\lambda \in(0,1)$

$$
P\left(\inf _{\|t\|>b}\left\|T_{n}\left(\beta(\lambda)+n^{-1 / 2} t ; \lambda\right)\right\|>u\right)>1-\epsilon
$$


Proof of (i):

We prove this step via Koul and Saleh (1995). By assumption $A 0, F_{0}$ is continuous hence we obtain $\hat{\boldsymbol{\beta}}_{n}(\lambda)$ as a unique solution of the minimization problem. Now use of Theorem 3.3 of Koenker and Bassett (1978) yields after writing $\operatorname{sgn}(x) \equiv 1-2 I(x \leq$ $0)+I(x=0)$, for each $\lambda \in(0,1)$ with probability one (w.p.1)

$$
\begin{aligned}
(\lambda-1) 1_{p+1}^{\prime}< & \sum_{i \in h^{c}} d_{i}^{\prime}\left\{I\left(y_{i}-d_{i}^{\prime} \hat{\beta}_{n}(\lambda) \leq 0\right)-\lambda\right\} D_{n}^{-1}(h) \\
& \quad+1 / 2 \sum_{i \in h^{c}} d_{i}^{\prime} I\left(y_{i}-d_{i}^{\prime} \hat{\beta}_{n}(\lambda)=0\right) D_{n}^{-1}(h)<\lambda 1_{p+1}^{\prime}
\end{aligned}
$$

where $D_{n}(h)$ is defined in section 3.2. Let $w_{n}^{\prime}(\lambda)$ denote the vector inside the inequalities, i.e

$$
(\lambda-1) 1_{p+1}^{\prime}<w_{n}^{\prime}(\lambda)<\lambda 1_{p+1}^{\prime} .
$$

Also, for each $i \in h$ by Theorem 3.2.1 we have

$$
\because \hat{\boldsymbol{S}}_{n}(\lambda)=d_{i}^{\prime} D_{n}^{-1}(h) Y(h)=\left(0, \cdots, \stackrel{i}{i}_{1}, \cdots, 0\right) Y(h)=y_{i}
$$

which implies that w.p.1

$$
I\left(y_{i}-d_{i}^{\prime} \hat{\beta}_{n}(\lambda)=0\right)=0 \quad \forall i \in h^{c} .
$$

Then, w.p.l we have

$$
\begin{aligned}
& {\left[\sum_{i=1}^{n} d_{i}\left\{I\left(y_{i}-d_{i}^{\prime} \hat{\beta}_{n}(\lambda) \leq 0\right)-\lambda\right\}\right.} \\
& \left.\quad-\sum_{i \in h} d_{i}^{\prime}\left\{I\left(y_{i}-d_{i}^{\prime} \hat{\beta}_{n}(\lambda) \leq 0\right)-\lambda\right\}\right] D_{n}^{-1}(h)=w_{n}^{\prime}(\lambda) .
\end{aligned}
$$

Note that (3.103) implies w.p. 1

$$
I\left(y_{i}-d_{i}^{\prime} \hat{\beta}_{n}(\lambda) \leq 0\right)=1 \quad \forall i \in h .
$$

Therefore by the definition of $T_{n}\left(\hat{\beta}_{n}(\lambda) ; \lambda\right)$, we have

$$
T_{n}\left(\hat{\beta}_{n}(\lambda) ; \lambda\right)-n^{-1 / 2} \sum_{i \in h} d_{i}(1-\lambda)=n^{-1 / 2} D_{n}^{\prime}(h) w_{n}(\lambda)
$$


which implies that

$$
\left\|T_{n}\left(\dot{\beta}_{n}(\lambda) ; \lambda\right)\right\| \leq n^{-1 / 2} \sum_{i \in h}\left\|d_{i}\right\|(1-\lambda)+n^{-1 / 2}\left\|D_{n}^{\prime}(h) w_{n}(\lambda)\right\| .
$$

From (3.101), we further obtain

$$
\left\|D_{n}^{\prime}(h) w_{n}(\lambda)\right\| \leq\left\|D_{n}^{\prime}(h) 1_{p+1}\right\|
$$

where

$$
D_{n}^{\prime}(h) 1_{p+1}=\left(\sum_{j=0}^{p} d_{0}, \cdots, \sum_{j=0}^{p} d_{p j}\right) .
$$

But, each component is bounded above by $\max _{i}\left\|d_{i}\right\| \sqrt{p+1}$ which implies

$$
n^{-1 / 2}\left\|D_{n}^{\prime}(h) \mathbf{1}_{p+1}\right\| \leq n^{-1 / 2} \max _{1}\left\|d_{i}\right\|(p+1)
$$

Also we have

$$
n^{-1 / 2} \sum_{i \in h}\left\|d_{i}\right\|(1-\lambda) \leq n^{-1 / 2} \max _{i}\left\|d_{i}\right\|(p+1) .
$$

Thus, we get w.p.1

$$
\left\|T_{n}\left(\hat{\beta}_{n}(\lambda) ; \lambda\right)\right\| \leq 2(p+1) n^{-1 / 2} \max _{i}\left\|d_{i}\right\|
$$

and the R.H.S converges to zero in probability by $\mathrm{Al}$.

Now, we prove step(ii),

Proof of (ii):

To show $(3.98)$, we look at the asymptotic distribution of $T_{n}(\beta(\lambda) ; \lambda)$. Since, $Z_{1}^{\prime} s$ are i.i.d we use the Hajek-Šidak C.L.T, Theorem 3.3.6 of Sen and Singer (1993). For this reason we check the condition which is satisfied by conditions A1 and A2, i.e

$$
\frac{n^{-1} \max _{i} d_{i j}^{2}}{n^{-1} \sum_{i=1}^{n} d_{i j}^{2}} \rightarrow 0 \text { as } n \rightarrow \infty
$$

which implies that for each $\lambda \in(0,1)$

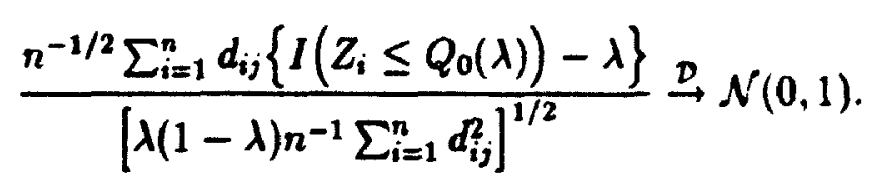


Therefore, this property implies that for every $\epsilon>0$ there exists an $M_{e}$ such that

$$
P\left(T_{n j}(\beta(\lambda) ; \lambda) \leq M_{c}\right)>1-2 \epsilon / 3 .
$$

We apply this result to prove step (iii).

Proof of (iii):

We prove this part via Koul and Saleh (1995). First, we define the following process for simplicity

$$
T_{n}^{*}(t ; \lambda)=T_{n}\left(\beta(\lambda)+n^{-1 / 2} t ; \lambda\right) .
$$

Now, every $t \in \mathbb{R}^{p+1}$ with $\|t\|>b$ could be written as $t=r \theta,|r|>b$ and $\|\theta\|=1$. Also note that $T_{n}^{* \prime}(r \theta ; \lambda) \cdot \theta$ is nondecreasing function of $r, \forall \theta \in \mathbb{E}^{p+1}$. Therefore, Cauchy-Schwarz inequality implies for each $\lambda \in(0,1)$

$$
\inf _{\|t\|>b}\left\|T_{n}^{*}(t ; \lambda)\right\|^{2} \geq \inf _{|r|>b, \| \theta_{\|=1}}\left\{T_{n}^{* \prime}(r \theta ; \lambda) \cdot \theta\right\}^{2} \geq \inf _{|r|=b, \| \theta_{\|=1}}\left\{T_{n}^{* \prime}(r \theta ; \lambda) \cdot \theta\right\}^{2}
$$

Now, let

$$
\tilde{T}_{n}^{*}(r, \theta ; \lambda)=T_{n}^{* \prime}(0 ; \lambda) \cdot \theta+q_{0}(\lambda) r \theta^{\prime} \Sigma_{n} \theta
$$

and

$$
\begin{aligned}
k_{n} & =\inf \left\{\theta^{\prime} \Sigma_{n} \theta,\|\theta\|=1\right\} \\
k & =\lim _{n} k_{n}=\inf \left\{\theta^{\prime} \Sigma \theta,\|\theta\|=1\right\} \\
A_{n}^{c} & =\left\{k(1-\epsilon) \leq k_{n} \leq(1+\epsilon) k\right] .
\end{aligned}
$$

Since $\boldsymbol{\Sigma}$ is positive definite we have $\boldsymbol{k}>0$. This with the result of step (ii), implies that for every $\epsilon>0$ there exists an $M_{\epsilon}, N_{1}^{e}$ and $N_{2}^{\epsilon}$ such that

$$
\left.P\left(A_{n}^{\epsilon}\right)=: \cdot k_{n}-k \mid<\epsilon k\right) \geq 1-\epsilon / 3, \quad \forall n \geq N_{1}^{e}
$$

and

$$
P\left(\sup _{\| \theta_{\|=1}}\left|T_{n}^{\prime \prime}(0 ; \lambda) \cdot \theta\right| \leq M_{\imath}\right) \geq 1-\epsilon / 3 . \quad \forall n \geq N_{2}^{\epsilon} .
$$


Also, by Lemma 3.4.2 $\exists$ an $N_{3}^{e}$ such that

$$
\begin{aligned}
& P\left(\inf _{|r|=b, \| \theta_{\|=1}}\left\{T_{n}^{\prime \prime}(r \theta ; \lambda) \cdot \theta\right\}^{2}>u\right) \\
& \quad \geq P\left(\inf _{|r|=6, \| \theta_{\|=1}}\left\{\tilde{T}_{n}^{*}(r, \theta ; \lambda)\right\}^{2}>u\right)-\epsilon / 3, \quad n \geq N_{3}^{e} .
\end{aligned}
$$

Now, we use the fact that ||$d|-| c|| \leq|d+c|, d, c \in \mathbb{R}$. Then, we have

$$
\begin{aligned}
& P\left(\inf _{|r|=b . \| \theta_{\|=1}}\left\{\tilde{T}_{n}^{*}(r, \theta ; \lambda)\right\}^{2}>u\right) \\
& \quad \geq P\left(|| T_{n}^{\prime \prime}\left(0 ; \lambda, \cdot \theta\left|-q_{0}(\lambda) b \theta^{\prime} \Sigma_{n} \theta\right|>u^{1 / 2} \quad \forall\|\theta\|=1\right)\right. \\
& \geq P\left(\left|T_{n}^{* \prime \prime}(0 ; \lambda) \cdot \theta\right| \leq-u^{-1 / 2}+b k_{n} q_{0}(\lambda) \quad \forall\|\theta\|=1\right) \\
& \geq P\left(\sup _{\| \theta_{\|=1}}\left|T_{n}^{* \prime}(0 ; \lambda) \cdot \theta\right| \leq-u^{-1 / 2}+b k(1-\epsilon) q_{0}(\lambda) ; A_{n}^{c}\right) \\
& \geq 1-(2 \epsilon / 3), \quad \forall n \geq n^{\prime}=N_{1}^{\prime \prime} \vee N_{2}^{c} \vee N_{3}^{c}
\end{aligned}
$$

by (3.120) and (3.121), as long as $b \geq \frac{\left(M_{1}+u^{1 / 2}\right)}{k(1-c)_{90}(\lambda)}$, which completes the proof of step (iii).

Now, we use the results (3.97) and (3.99), to prove the Lemma similar to Jurecková (1984) by defining

$$
t=n^{1 / 2}\left(\hat{\beta}_{n}(\lambda)-\beta(\lambda)\right)
$$

Thus, for all $\epsilon>0,0<u<\infty \exists b\left(=b_{\epsilon}\right)>0$ and $N^{k}$ so that

$$
\begin{aligned}
& P\left(n^{1 / 2}\left\|\hat{\beta}_{n}(\lambda)-\beta(\lambda)\right\|>b\right) \\
& =P\left(n^{1 / 2}\left\|\hat{\beta}_{n}(\lambda)-\beta(\lambda)\right\|>b,\left\|T_{n}\left(\beta(\lambda)+n^{-1 / 2} t ; \lambda\right)\right\|<u\right) \\
& \quad+P\left(n^{1 / 2}\left\|\hat{\beta}_{n}(\lambda)-\beta(\lambda)\right\|>b,\left\|T_{n}\left(\beta(\lambda)+n^{-1 / 2} t ; \lambda\right)\right\| \geq u\right) \\
& \leq P\left(\inf _{\|\ell\|>b}\left\|T_{n}\left(\beta(\lambda)+n^{-1 / 2} t ; \lambda\right)\right\|<u\right)+P\left(\left\|T_{n}\left(\hat{\beta}_{n}(\lambda) ; \lambda\right)\right\| \geq u\right) \\
& =\epsilon / 2+\epsilon / 2=\epsilon .
\end{aligned}
$$


Therefore, the proof of Lemma 3.4.2 is complete.

Proof of Theorem 3.4.1

It follows from Lemma 3.4 .2 that

$$
T_{n}\left(\beta(\lambda)+n^{-1 / 2} t ; \lambda\right)-T_{n}(\beta(\lambda) ; \lambda)-\Sigma_{n} t q_{0}(\lambda)=o_{P}(1)
$$

for ev - $y$ sequence of random vector $t$, such that $\|t\|=O_{p}(1)$. Hence, by substituting $t$ with $n^{1 / 2}\left(\hat{\beta}_{n}(\lambda)-\beta(\lambda)\right)$ we have

$$
\begin{gathered}
T_{n}\left(\beta(\lambda)+n^{-1 / 2}\left[n^{1 / 2}\left(\hat{\beta}_{n}(\lambda)-\beta(\lambda)\right)\right] ; \lambda\right)-T_{n}(\beta(\lambda) ; \lambda) \\
-q_{0}(\lambda) \Sigma_{n}\left[n^{1 / 2}\left(\hat{\beta}_{n}(\lambda)-\beta(\lambda)\right)\right]=o_{P}(1) .
\end{gathered}
$$

On the other hand

$$
T_{n}\left(\hat{\beta}_{n}(\lambda) ; \lambda\right)-T_{n}(\beta(\lambda) ; \lambda)-q_{0}(\lambda) \Sigma_{n}\left[n^{1 / 2}\left(\hat{\beta}_{n}(\lambda)-\beta(\lambda)\right)\right]=o_{P}(1)
$$

But, by (3.97) we have $T_{n}\left(\hat{\boldsymbol{\beta}}_{n}(\lambda) ; \lambda\right)=$ op (1). Therefore, the proof of Theorem 3.4.1 is complete.

The following Corollaries give the asymptotic distribution of RQ's.

Corollary 3.4.4 Assume the conditions of theorem 3.4.1 satisficd. Then,

$$
n^{1 / 2}\left(\hat{\beta}_{n}(\lambda)-\beta(\lambda)\right) \stackrel{\mathcal{D}}{\rightarrow} \mathcal{N}_{p+1}\left(0, \frac{\lambda(1-\lambda)}{q_{0}^{2}(\lambda)} \Sigma^{-1}\right)
$$

Corollary 3.4.5 Let the conditions A0-A2 hold. Then, for each $\lambda_{1}, \cdots, \lambda_{k}$ such that $0<\lambda_{1}<\cdots<\lambda_{k}<1$ the asymptotic joint distribution of the regression quantiles is given by

$$
\left[n^{1 / 2}\left(\hat{\beta}_{n}\left(\lambda_{1}\right)-\beta\left(\lambda_{1}\right)\right), \cdots, n^{1 / 2}\left(\hat{\beta}_{n}\left(\lambda_{k}\right)-\beta\left(\lambda_{k}\right)\right)\right] \stackrel{\mathcal{D}}{\rightarrow} \mathcal{N}_{p+1 \times k}\left(0, \Omega \otimes \Sigma^{-1}\right)
$$

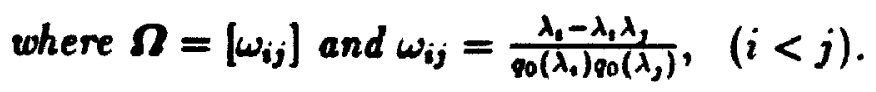




\section{Proof}

By Theorem 3.4.1 we write

$$
\begin{aligned}
& {\left[n^{1 / 2}\left(\hat{\beta}_{n}\left(\lambda_{1}\right)-\beta\left(\lambda_{1}\right)\right), \ldots, n^{1 / 2}\left(\hat{\beta}_{n}\left(\lambda_{k}\right)-\beta\left(\lambda_{k}\right)\right)\right]=} \\
& \quad-\left\{\frac{1}{q_{0}\left(\lambda_{1}\right)}, \ldots, \frac{1}{q_{0}\left(\lambda_{k}\right)}\right\} \otimes \Sigma_{n}^{-1}\left[T_{n}\left(\beta\left(\lambda_{1}\right) ; \lambda_{1}\right), \ldots, T_{n}\left(\beta\left(\lambda_{k}\right) ; \lambda_{k}\right)\right]
\end{aligned}
$$

and then by corollary 3.4.4 and Cramer-Wold device we get the result.

Now, we state the main Theorem of TLSE similar to Jureckková (1984).

Theorem 3.4.6 Assume that the conditions A0, AI and A2 hold. Then, for each $\lambda_{1}$ and $\lambda_{2}$ such that $0<\lambda_{1}<1 / 2<\lambda_{2}<1$

$$
\begin{aligned}
& \text { i) } \quad n^{1 / 2}\left(\bar{\beta}_{n}\left(\lambda_{1}, \lambda_{2}\right)-\beta\right) \\
& n^{-1 / 2}\left\{\left(\lambda_{2}-\lambda_{1}\right) \Sigma_{n}\right\}^{-1} \sum_{i=1}^{n} d_{i}\left(\phi\left(Z_{i}\right)-\gamma\right)+o_{P}(1) \\
& \text { ii) } \quad n^{1 / 2}\left(\bar{\beta}_{n}\left(\lambda_{1}, \lambda_{2}\right)-\beta\right) \stackrel{D}{\rightarrow} \mathcal{N}_{p+1}\left(0, \sigma^{2}\left(\lambda_{1}, \lambda_{2}\right) \Sigma^{-1}\right)
\end{aligned}
$$

where $\phi\left(Z_{i}\right), \gamma$ and $\sigma^{2}\left(\lambda_{1}, \lambda_{2}\right)$ are as defined in (2.97) and (2.98) respectively.

\section{Proof}

This Theorem will be proved also with the aid of two Lemmas. First. we define the following processes

$$
\tilde{T}_{n}\left(t_{i} \lambda\right)=n^{-1 / 2} \sum_{i=1}^{n} d_{i} Z_{1} I\left(Z_{1} \leq Q_{0}(\lambda)+n^{-1 / 2} d_{i}^{\prime} t\right)
$$

and

$$
\begin{aligned}
& \tilde{T}_{n}^{0}(t ; \lambda)= \tilde{T}_{n}(t ; \lambda)-E\left(\dot{T}_{n}(t ; \lambda)\right) \\
&=n^{-1 / 2} \sum_{i=1}^{n} d_{i}\left\{Z_{i} I\left(Z_{i} \leq Q_{0}(\lambda)+n^{-1 / 2} d_{i}^{\prime} t\right)\right. \\
&\left.-\mu\left(Q_{0}(\lambda)+n^{-1 / 2} d_{i}^{\alpha} t\right)\right\}
\end{aligned}
$$

where $\mu(\cdot)$ is defined in (2.103). Then, 
Lemma 3.4.7 Under the condition of Theorem 3.4.6 for each $b>0$

$$
\sup _{\|t\| \leq b}\left\|\tilde{T}_{n}(t ; \lambda)-\tilde{T}_{n}(0 ; \lambda)-g_{0}(\lambda) Q_{0}(\lambda) \Sigma_{n} t\right\|=O_{P}(1) .
$$

\section{Proof}

Consider $\tilde{T}_{n j}(t ; \lambda), j=0, \ldots, p$ as a $j^{\text {th }}$ component of $\tilde{T}_{n}(t ; \lambda)$. Then, we have

$$
\begin{aligned}
& \sup _{\|\| \| \leq b}\left|\tilde{T}_{n j}(t ; \lambda)-\tilde{T}_{n j}(0 ; \lambda)-q_{0}(\lambda) Q_{0}(\lambda) \Sigma_{n} t\right| \\
& \leq \sup _{\|t\| \leq b}\left|\tilde{T}_{n j}^{0}(t ; \lambda)-\tilde{T}_{n j}^{0}(0 ; \lambda)\right| \\
& \quad+\sup _{\|t\| \leq b}\left|E\left(\tilde{T}_{n j}(t ; \lambda)\right)-E\left(\tilde{T}_{n j}(0 ; \lambda)\right)-\varphi_{0}(\lambda) Q_{0}(\lambda) \Sigma_{n} t\right| \\
& =J_{1}+J_{2} .
\end{aligned}
$$

First, note that

$$
J_{2} \leq n^{-1 / 2} \sum_{i=1}^{n}\left|d_{i j}\right| \sup _{\|t\| \leq b}\left|\mu\left(Q_{0}(\lambda)+n^{-1 / 2} d_{i}^{\prime} t\right)-\mu\left(Q_{0}(\lambda)\right)-q_{0}(\lambda) Q_{0}(\lambda) n^{-1 / 2} d_{i}^{\prime} t\right|
$$

Then, by the mean value Theorem there is a $\Delta(t) \in\left(0, n^{-1 / 2} d_{i}^{\prime} t\right)$ such that

$$
\begin{aligned}
J_{2} & \leq b n^{-1} \sum_{i=1}^{n}\left|d_{i j}\right|\left\|d_{i}\right\| \sup _{\|t\| \leq b} \mid\left(Q_{0}(\lambda)+\Delta(t)\right) f_{0}\left(Q_{0}(\lambda)+\Delta(t)\right) \\
& =0(1) .
\end{aligned}
$$

Since, $\|t\| \leq b$ implies that $\Delta(t) \leq \delta_{n}=n^{-1 / 2}\left\|d_{i}\right\| b$ then, by conditions $A 0$ and A2 we get the result. To prove Lemma 3.4.7 it is enough to show that $J_{1}=o_{P}(1)$ where

$$
J_{1}=\sup _{\|t\| \leq b}\left|\tilde{T}_{n j}^{0}(t ; \lambda)-\tilde{T}_{n j}^{0}(0 ; \lambda)\right|
$$

First, we consider for each $t \in \mathcal{N}(b)$

$$
\left|\tilde{T}_{n j}^{0}(\mathrm{t} ; \lambda)-\tilde{T}_{n j}^{0}(0 ; \lambda)\right|
$$




$$
\begin{aligned}
& =\mid n^{-1 / 2} \sum_{i=1}^{n} d_{i j}\left\{Z _ { i } I \left(Z_{i} \leq\left(Q_{0}(\lambda)+n^{-1 / 2} d_{i}^{\prime} t\right)-\mu\left(Q_{0}(\lambda)+n^{-1 / 2} d_{i}^{\prime} t\right)\right.\right. \\
& \left.\quad-Z_{i} I\left(Z_{i} \leq Q_{0}(\lambda)\right)+\mu\left(Q_{0}(\lambda)\right)\right\} \mid \\
& =\left|n^{-1 / 2} \sum_{i=1}^{n} d_{i j}\left\{\dot{V}_{i}(t)-\bar{\nu}_{n i}(t)\right\}\right|
\end{aligned}
$$

where

$$
\begin{aligned}
& \dot{V}_{i}(\mathrm{t})=Z_{i} I\left(Q_{0}(\lambda)<Z_{i} \leq Q_{0}(\lambda)+n^{-1 / 2} d_{i}^{\prime} \mathrm{t}\right) \\
& \dot{\nu}_{n t}(\mathrm{t})=\mu\left(Q_{0}(\lambda)+n^{-1 / 2} d_{i}^{\nu} \mathrm{t}\right)-\mu\left(Q_{0}(\lambda)\right) .
\end{aligned}
$$

Now, we apply Chebyshev inequality to show that (3.141) goes to zero in probability.

$$
\begin{aligned}
& P\left(\left|n^{-1 / 2} \sum_{i=1}^{n} d_{i j}\left\{\tilde{V}_{i}(t)-\dot{V}_{n t}(t)\right\}\right|^{2}>\epsilon^{2}\right) \\
& \leq \frac{1}{c^{2}} \frac{1}{n} E\left(\sum_{i=1}^{n} d_{i j}\left\{\dot{V}_{i}(t)-\dot{\nu}_{n t}(t)\right\}\right)^{2} \\
& \frac{1}{\epsilon^{2}} \frac{1}{n} \sum_{i=1}^{n} d_{i j}^{2} E\left(\tilde{V}_{i}(t)-\dot{\nu}_{n t}(t)\right)^{2} \\
& \leq \epsilon \text { as } n \rightarrow \infty \text { by condition } A 0 \text { and } A 2 .
\end{aligned}
$$

Therefore, $J_{1}=o_{P}(1)$ for fixed $t$. For the tightness. we follow similar argument as in expression (3.8i) and use the compactness property of $\mathcal{N}(b)$. So, it is necessary to show that

$\forall \in>0 \exists \delta>0 \ni \forall s \in \mathcal{N}(b)$

$$
\limsup _{n} P\left(\sup _{\|t-s\| \leq b}\left|\tilde{T}_{n j}^{0}(t ; \lambda)-\tilde{T}_{n j}^{0}(s ; \lambda)\right|>2 \epsilon\right)<\epsilon .
$$

In this case we have

$$
\begin{aligned}
& \left|\tilde{T}_{n j}^{0}(\mathrm{t} ; \lambda)-\tilde{T}_{n j}^{0}(s ; \lambda)\right| \leq \\
& \quad n^{-1 / 2} \sum_{i=1}^{n}\left|d_{i j} Z_{i}\right|\left|I\left(Z_{i} \leq Q_{0}(\lambda)+n^{-1 / 2} d_{i}^{\prime} t\right)-I\left(Z_{i} \leq Q_{0}(\lambda)+n^{-1 / 2} d_{i}^{\prime} s\right)\right| \\
& \quad+n^{-1 / 2} \sum_{i=1}^{n}\left|d_{i j}\right|\left|\mu\left(Q_{0}(\lambda)+n^{-1 / 2} d_{i}^{\prime} t\right)-\mu\left(Q_{0}(\lambda)+n^{-1 / 2} d_{i}^{\prime} s\right)\right|
\end{aligned}
$$


By inequality (3.93) the above expression is bounded aboie by

$$
\begin{aligned}
& n^{-1 / 2} \sum_{i=1}^{n}\left|d_{i j} Z_{i}\right|\left\{I\left(Z_{i} \leq Q_{0}(\lambda)+n^{-1 / 2} d_{i}^{*}=+n^{-1 / 2}\left\|d_{i}\right\| \delta\right)\right. \\
& \left.-I\left(Z_{i} \leq Q_{0}(\lambda)+n^{-1 / 2} d_{i}^{\prime} \bullet-n^{-1 / 2}\left\|d_{i}\right\| \delta\right)\right\} \\
& +n^{-1 / 2} \sum_{i=1}^{n}\left|d_{i j}\right|\left|\mu\left(Q_{0}(\lambda)+n^{-1 / 2} d_{i}^{\prime} t\right)-\mu\left(Q_{0}(\lambda)+n^{-1 / 2} d_{i}^{\prime} \cdot\right)\right| \\
& =J_{11}+J_{12} .
\end{aligned}
$$

By the mean value Theorem and the same argument as in (3.139) we have

$$
\sup _{\|t-s\| \leq \delta} J_{12}=o(1)
$$

To show $\sup _{\|t-\varepsilon\| \leq \delta} J_{11}=o p(1)$ we note that $J_{11}$ does not depend on $t$ and so it is enough to show that $J_{11}=o_{P}(1)$. For this we center $J_{11}$ to obtain

$$
\begin{gathered}
J_{11}=n^{-1 / 2} \sum_{i=1}^{n}\left|d_{i j}\right|\left\{|| Z_{i} \mid I\left(Z_{i} \leq Q_{0}(\lambda)+n^{-1 / 2} d_{i}^{\prime} s+n^{-1 / 2}\left\|d_{i}\right\| \delta\right)\right. \\
\left.-\mu^{*}\left(Q_{0}(\lambda)+n^{-1 / 2} d_{i}^{\prime} s+n^{-1 / 2}\left\|d_{i}\right\| \delta\right)\right] \\
-\left[I\left(Z_{i} \leq Q_{0}(\lambda)+n^{-1 / 2} d_{i}^{\prime} s-n^{-1 / 2}\left\|d_{i}\right\| \delta\right)\right. \\
\left.\left.-\mu^{*}\left(Q_{0}(\lambda)+n^{-1 / 2} d_{i}^{\prime} s-n^{-1 / 2}\left\|d_{i}\right\| \delta\right)\right]\right\} \\
+n^{-1 / 2} \sum_{i=.}^{n}\left|d_{i j}\right|\left\{\mu^{*}\left(Q_{0}(\lambda)+n^{-1 / 2} d_{i}^{\prime} s+n^{-1 / 2}\left\|d_{i}\right\| \delta\right)\right. \\
\left.-\mu^{*}\left(Q_{0}(\lambda)+n^{-1 / 2} d_{i}^{\prime} s-n^{-1 / 2}\left\|d_{i}\right\| \delta\right)\right\} .
\end{gathered}
$$

By the same argument as we did for fixed $t$ in $J_{1}$, the first term of R.H.S of (3.148) goes to zero in probability. The second term is also less than $\epsilon$ by continuity of $\mu^{*}$ for sufficient small $\delta$. Therefore, the proof of lemma is complete.

Before we state the next lemma it is necessary to introduce the following processes,namely

$$
U_{j k}(t)=n^{-1} \sum_{i=1}^{n} d_{i j} d_{i k} I\left(Z_{i} \leq Q_{0}(\lambda)+n^{-1 / 2} d_{i}^{\prime} t\right)
$$


and

$$
U_{j k}^{0}(t)=U_{j k}(t)-E\left[U_{j k}(t)\right]
$$

Then,

Lemma 3.4.8 Under the conditions of Theorem 9.4.6, for $0<b<\infty$ and $\lambda \in$ $(0,1)$

$$
\sup _{\|t\| \leq b}\left|U_{j k}(t)-\lambda \sigma_{j k}\right| \stackrel{P}{\rightarrow} 0
$$

where $\sigma_{j k}$ is the $j \times k^{\text {th }}$ element of $\boldsymbol{\Sigma}$.

\section{Proof}

First, we prove this lemma for each $t$. Then,

$$
\begin{aligned}
& \left|U_{j k}(\mathrm{t})-\lambda \sigma_{j k}\right| \\
& =\left|n^{-1} \sum_{i=1}^{n} d_{i j} d_{i k} I\left(Z_{i} \leq Q_{0}(\lambda)+n^{-1 / 2} d^{\prime} . t\right)-\lambda \sigma_{j k}\right| \\
& =\mid n^{-1} \sum_{i=1}^{n} d_{i j} d_{i k}\left\{I\left(Z_{i} \leq Q_{0}(\lambda)+n^{-1 / 2} d_{i}^{\prime} t\right)-F_{0}\left(Q_{0}(\lambda)+n^{-1 / 2} d_{i}^{\prime} t\right)\right. \\
& \left.\quad+F_{0}\left(Q_{0}(\lambda)+n^{-1 / 2} d_{i}^{\prime} t\right)\right\}-,_{j k} F_{0}\left(Q_{0}(\lambda)\right) \mid \\
& \leq\left|r^{-1} \sum_{i=1}^{n} d_{i j} d_{i k}\left\{I\left(Z_{i} \leq Q_{0}(\lambda)+n^{-1 / 2} d_{i}^{\prime} t\right)-F_{0}\left(Q_{0}(\lambda)+n^{-1 / 2} d_{i}^{\prime} t\right)\right\}\right| \\
& +\left|n^{-1} \sum_{i=1}^{n} d_{i j} d_{i k} F_{0}\left(Q_{0}(\lambda)+n^{-1 / 2} d_{i}^{\prime} t\right)-\sigma_{j k} F_{0}\left(Q_{0}(\lambda)\right)\right| \\
& =I_{1}+I_{2}
\end{aligned}
$$

First we show that $I_{1}=o_{P}(1)$ that is to say that for every $\epsilon>0$, we have

$$
\begin{gathered}
P\left\{\left|n^{-1} \sum_{i=1}^{n} d_{i j} d_{i k}\left\{I\left(Z_{i} \leq Q_{0}(\lambda)+n^{-1 / 2} d_{i}^{\prime} t\right)-F_{0}\left(Q_{0}(\lambda)+n^{-1 / 2} d_{i}^{\prime} t\right)\right\}\right|^{2}>\epsilon^{2}\right\} \\
\leq \frac{1}{\epsilon^{2}} \frac{1}{n^{2}} E\left\{\sum_{i=1}^{n} d_{i j} d_{i k}\left\{I\left(Z_{i} \leq Q_{0}(\lambda)+n^{-1 / 2} d_{i}^{\prime} t\right)-F_{0}\left(Q_{0}(\lambda)+n^{-1 / 2} d_{i}^{\prime} t\right)\right\}\right\}^{2} \\
=\frac{1}{\epsilon^{2}} \frac{1}{n^{2}} \sum_{i=1}^{n} d_{i j}^{2} d_{i k}^{2} E\left\{I\left(Z_{i} \leq Q_{0}(\lambda)+n^{-1 / 2} d_{i}^{\prime} t\right)-F_{0}\left(Q_{0}(\lambda)+n^{-1 / 2} d_{i}^{\prime} t\right)\right\}^{2}
\end{gathered}
$$




$$
\begin{aligned}
& \leq \frac{1}{\epsilon^{2}} \frac{1}{n^{2}} \sum_{i=1}^{n} d_{i j}^{2} d_{i k}^{2} \\
& \leq \frac{1}{\epsilon^{2}}\left(n^{-1 / 2} \max _{i, j}\left|d_{i j}\right|\right)^{2} \cdot n^{-1} \sum_{i=1}^{n} d_{3 k}^{2} \\
& \leq \epsilon \text { as } n \rightarrow \infty \text { by } A 1 \text { and } A 2 .
\end{aligned}
$$

Continuity of $F_{0}$ together with C-S inequality and condition A2 implies that $I_{2}=$ $o(1)$. Therefore, we have the result for fixed $t$. For tightness of $I_{1}$ we follow the proof of the Lenıma 3.4.2. Next we ronsider $I_{2}$. For this we have

$$
\begin{aligned}
\sup _{\|t\| \leq b} I_{2}= & \left.\sup _{\|t\| \leq b} \mid n^{-1} \sum_{i=1}^{n} d_{i j} d_{i k} F_{0}\left(Q_{0}(\lambda)\right)+n^{-1 / 2} d_{i}^{\prime} \mathrm{t}\right)-\sigma_{j k} F_{0}\left(Q_{0}(\lambda)\right) \mid \\
\leq & \sup _{\|t\| \leq b}\left|n^{-1} \sum_{i=1}^{n} d_{i j} d_{i k}\left[F_{0}\left(Q_{0}(\lambda)+n^{-1 / 2} d_{i}^{\prime} \mathbf{t}\right)-F_{0}\left(Q_{0}(\lambda)\right)\right]\right| \\
& \quad+\Gamma_{0}\left(Q_{0}(\lambda)\right)\left|n^{-1} \sum_{i=1}^{n} d_{i j} d_{i k}-\sigma_{j k}\right| \\
\leq & n^{-1} \sum_{i=1}^{n}\left|d_{i j} d_{i k}\right| \sup _{\|t\| \leq b} \mid F_{0}\left(Q_{0}\left(\lambda !+n^{-1 / 2} d_{i}^{\prime} \mathrm{t}\right)-F_{0}\left(Q_{0}(\lambda)\right) \mid+o(1)\right. \\
\leq & \left(n^{-1} \sum_{i=1}^{n} d_{i j}^{2}\right)^{1 / 2}\left(n^{-1} \sum_{i=1}^{n} d_{i k}^{2}\right)^{1 / 2} \cdot o(1)+o(1) \\
\leq & \epsilon \text { as } n \rightarrow \infty \text { by C-S inequality and A0 and A2. }
\end{aligned}
$$

Now we change the process $U_{j k}(\cdot)$ to

$$
U_{j k}^{*}(\mathbf{t})=n^{-1} \sum_{i=1}^{n} d_{\cdot j} d_{i k} I\left(Q_{0}\left(\lambda_{1}\right)+n^{-1 / 2} d_{i}^{\prime} \mathbf{t}<Z_{\mathbf{t}} \leq Q_{0}\left(\lambda_{2}\right): n^{-1 / 2} d_{i}^{\prime} \mathbf{t}\right)
$$

for each $\lambda_{1}, \lambda_{2} \ni 0<\lambda_{1}<\lambda_{2}<1$. Then, we have

$$
\sup _{\|t\| \leq b}\left|U_{j k}^{*}(t)-\left(\lambda_{2}-\lambda_{1}\right) \sigma_{i k}\right|=o_{P}(1)
$$

We apply (3.156) to prove Theorem 3.4.6.

Proof of Theorem 3.4.6 (i)

By using the result of Lemma 3.4.8 in the matrix form we have

$$
n^{-1} D_{n}^{\prime} A D_{n}=n^{-1} \sum_{i=1}^{n} d_{i} d_{i}^{\prime} J\left(Q_{0}\left(\lambda_{1}\right)+n^{-1 / 2} d_{i}^{\prime} t<Z_{i} \leq Q_{0}\left(\lambda_{2}\right)+n^{-1 / 2} d_{i}^{\prime} t\right)
$$




$$
=\left(\lambda_{2}-\lambda_{1}\right) \Sigma+o_{P}(1) \text { as } n \rightarrow \infty \text {. }
$$

By the structure of matrix $A$,

$$
\begin{aligned}
n^{-1 / 2} D_{n}^{\prime} A Z= & n^{-1 / 2} \sum_{i=1}^{n} d_{i}\left\{I\left(Z_{i} \leq Q_{0}\left(\lambda_{2}\right)+d_{i}^{\prime}\left(\hat{\beta}_{n}\left(\lambda_{2}\right)-\beta\left(\lambda_{2}\right)\right)\right)\right. \\
& \left.-I\left(Z_{i} \leq Q_{0}\left(\lambda_{1}\right)+d_{i}^{\prime}\left(\hat{\beta}_{n}\left(\lambda_{1}\right)-\beta\left(\lambda_{1}\right)\right)\right)\right\} Z_{1} \\
= & \tilde{T}_{n}\left(t_{2} ; \lambda_{2}\right)-\tilde{T}_{n}\left(t_{1} ; \lambda_{1}\right)
\end{aligned}
$$

where

$$
\mathbf{t}_{i}=n^{1 / 2}\left(\hat{\boldsymbol{\beta}}_{n}\left(\lambda_{1}\right)-\boldsymbol{\beta}\left(\lambda_{1}\right)\right) \quad i=1,2
$$

But.

$$
\begin{aligned}
\tilde{\boldsymbol{T}}_{n}\left(\mathbf{t}_{2} ; \lambda_{2}\right)-\tilde{\boldsymbol{T}}_{n}\left(\mathbf{t}_{1} ; \lambda_{1}\right)= & {\left[\tilde{\boldsymbol{T}}_{n}\left(\mathbf{t}_{2} ; \lambda_{2}\right)-\tilde{\boldsymbol{T}}_{n}\left(\mathbf{0} ; \lambda_{2}\right)-\boldsymbol{q}_{0}\left(\lambda_{2}\right) Q_{0}\left(\lambda_{2}\right) \Sigma_{n} \mathbf{t}_{2}\right] } \\
& -\left[\tilde{\boldsymbol{T}}_{n}\left(\mathbf{t}_{1} ; \lambda_{1}\right)-\tilde{\boldsymbol{T}}_{n}\left(\mathbf{0} ; \lambda_{1}\right)-q_{0}\left(\lambda_{1}\right) Q_{0}\left(\lambda_{1}\right) \Sigma_{n} \mathbf{t}_{1}\right] \\
& +\left[\tilde{\boldsymbol{T}}_{n}\left(\mathbf{0} ; \lambda_{2}\right)-\tilde{\boldsymbol{T}}_{n}\left(\mathbf{0} ; \lambda_{1}\right)+\boldsymbol{q}_{0}\left(\lambda_{2}\right) Q_{0}\left(\lambda_{2}\right) \boldsymbol{\Sigma}_{n} \mathbf{t}_{2}\right. \\
& \left.-q_{0}\left(\lambda_{1}\right) Q_{0}\left(\lambda_{1}\right) \boldsymbol{\Sigma}_{n} \mathbf{t}_{1}\right]
\end{aligned}
$$

By Lemma 3.4.7 the first and second terms of (3.160) are of (1). Also, by substituting $t_{i}$ with its representation given in the Theorem 3.4 .1 we have

$$
\begin{aligned}
n^{-1 / 2} D_{n}^{\prime} \boldsymbol{A Z} & =\boldsymbol{T}_{n}\left(\boldsymbol{\beta}\left(\lambda_{1}\right) ; \lambda_{1}\right) Q_{0}\left(\lambda_{1}\right)-\boldsymbol{T}_{n}\left(\boldsymbol{\beta}\left(\lambda_{2}\right) ; \lambda_{2}\right) Q_{0}\left(\lambda_{2}\right) \\
& +n^{-1 / 2} \sum_{i=1}^{n} \boldsymbol{d}_{1} Z_{1}\left\{I\left(Q_{0}\left(\lambda_{1}\right)<Z_{1} \leq Q_{0}\left(\lambda_{2}\right)\right)\right\}+u_{P}(1)(3.1
\end{aligned}
$$

and finally by the definition of $\boldsymbol{T}_{n}(\beta(\lambda) ; \lambda)$ and using the fact that $I\left(Z_{i} \leq a\right)=$ $1-I\left(Z_{i}>a\right)$ we have

$$
\begin{aligned}
n^{-1 / 2} D_{n}^{\prime} A Z= & n^{-1 / 2} \sum_{i=1}^{n} d_{i}\left\{I\left(Z_{1} \leq Q_{0}\left(\lambda_{1}\right)\right) Q_{0}\left(\lambda_{1}\right)+Z_{1} I\left(Q_{0}\left(\lambda_{1}\right)<Z_{i} \leq Q_{0}\left(\lambda_{2}\right)\right)\right. \\
& \left.+I\left(Z_{i}>Q_{0}\left(\lambda_{2}\right)\right) Q_{0}\left(\lambda_{3}\right)-\lambda_{1} Q_{0}\left(\lambda_{1}\right)-\left(1-\lambda_{2}\right) Q_{0}\left(\lambda_{2}\right)\right\}+o_{P}(1) \\
= & n^{-1 / 2} \sum_{i=1}^{n} d_{i}\left\{\phi\left(Z_{1}\right)-\gamma\right\}+o_{P}(1)
\end{aligned}
$$


where $\phi\left(Z_{i}\right)$ and $\gamma$ are given in the (2.97). Finally we have

$$
\left(D_{n}^{\prime} A D_{n}\right)^{-1} D_{n}^{\prime} A Z=\left(D_{n}^{\prime} A D_{n}\right)^{-1} D_{n}^{\prime} A Y-\beta
$$

or

$$
\left(n^{-1} D_{n}^{\prime} A D_{n}\right)^{-1} n^{-1 / 2} D_{n}^{\prime} A Z=n^{1 / 2}\left(\bar{\beta}_{n}\left(\lambda_{1}, \lambda_{2}\right)-\beta\right)
$$

As a result

$$
n^{1 / 2}\left(\bar{\beta}_{n}\left(\lambda_{1}, \lambda_{2}\right)-\beta\right)=\left(\left(\lambda_{2}-\lambda_{1}\right) \Sigma_{n}\right)^{-1} n^{-1 / 2} \sum_{i=1}^{n} d_{i}\left\{\phi\left(Z_{i}\right)-\gamma\right\}+o_{P}(1) \text {. }
$$

Therefore, $t \mathrm{~h}$. proof of the first part of Theorem 3.4.6 is complete.

Proof of Theorem 3.4.6 (ii)

The result of the second part is given by using the result of part (i), considering $F_{0}$ symmetric with $\lambda_{1}=1-\lambda_{2}$. In this case we have $\gamma=0$ and $E\left(\phi\left(Z_{1}\right)\right)=0$. Thus, by similar argument as (3.114) we have

$$
\frac{n^{-1 / 2} \sum_{i=1}^{n} d_{i j} \phi\left(Z_{i}\right)}{\left[\sigma^{2}\left(\lambda_{1}, \lambda_{2}\right) n^{-1} \sum_{i=1}^{n} d_{i j}^{2}\right]^{1 / 2}} \stackrel{D}{\rightarrow} \mathcal{N}(0,1)
$$

and so W.L.G. the proof of the second part is complete.

As we saw in the result of theorem 3.4.6, TLSE converges to normal distribution with variance $\sigma^{2}\left(\lambda_{1}, \lambda_{2}\right) \Sigma^{-1}$. Here we define a consistent estimator of $\sigma^{2}\left(\lambda_{1}, \lambda_{2}\right)$. Let $Y_{1}, Y_{2}, \ldots, Y_{n}$ be sample from regression model (3.65). Then the estimation of $\sigma^{2}\left(\lambda_{1}, \lambda_{2}\right)$ is given by

$$
\begin{aligned}
S_{n}^{2}\left(\lambda_{1}, \lambda_{2}\right)= & \left(\lambda_{2}-\lambda_{1}\right)^{-2}\left\{(n-1)^{-1} \sum_{i=1}^{n}\left(Y_{i}-\delta_{n}\right)^{2} I\left(\hat{\beta}_{n}\left(\lambda_{1}\right)<Y_{i} \leq \hat{\beta}_{n}\left(\lambda_{2}\right)\right)\right. \\
& +\lambda_{1}\left(Q_{n}\left(\lambda_{1}\right)-\delta_{n}\right)^{2}+\left(1-\lambda_{2}\right)\left(Q_{n}\left(\lambda_{2}\right)-\delta_{n}\right)^{2} \\
& \left.-\left[\lambda_{1}\left(Q_{n}\left(\lambda_{1}\right)-\delta_{n}\right)+\left(1-\lambda_{2}\right)\left(Q_{n}\left(\lambda_{2}\right)-\delta_{n}\right)\right]^{2}\right\}
\end{aligned}
$$

where

$$
\begin{aligned}
\delta_{n} & =\left(\lambda_{1}-\lambda_{1}\right)^{-1} n^{-1} \sum_{i=1}^{n} Y_{i} I\left(d_{i}^{\prime} \hat{\beta}_{n}\left(\lambda_{1}\right)<Y_{i} \leq d_{i}^{\prime} \hat{\beta}_{n}\left(\lambda_{2}\right)\right) \\
& =c n^{-1} \sum_{i=1}^{n} Y_{i} I\left(Q_{0}\left(\lambda_{1}\right)+n^{-1 / 2} d_{i}^{\prime} t_{1}<Z_{i} \leq Q_{0}\left(\lambda_{2}\right)+n^{-1 / 2} d_{i}^{\prime} t_{2}\right)
\end{aligned}
$$


and $\mathbf{t}_{i}=n^{1 / 2}\left(\hat{\boldsymbol{\beta}}_{n}\left(\lambda_{i}\right)-\boldsymbol{\beta}\left(\lambda_{i}\right)\right)$. The following theorem states the consistency of $S_{n}^{2}\left(\lambda_{1}, \lambda_{2}\right)$.

Theorem 3.4.9 Let the conditions of Theorem 3.4.6 hold. Then,

$$
S_{n}^{2}\left(\lambda_{1}, \lambda_{2}\right) \stackrel{P}{\rightarrow} \sigma^{2}\left(\lambda_{1}, \lambda_{2}\right)
$$

Proof:

To prove this theorem first we shall show that

$$
\delta_{n} \stackrel{p}{\rightarrow} \delta
$$

where

$$
\delta=\left(\lambda_{2}-\lambda_{1}\right)^{-1} \int_{Q\left(\lambda_{1}\right)}^{Q\left(\lambda_{2}\right)} Y d F(y)=d^{\prime} \beta+\delta_{0}
$$

and $\delta_{0}$ is given in (2.99). Equivalently, it is necessary to show that

$$
\delta_{0 n} \stackrel{P}{\rightarrow} \delta_{0}
$$

where

$$
\delta_{0 n}=c n^{-1} \sum_{i=1}^{n} Z_{1} I\left(Q_{0}\left(\lambda_{1}\right)+n^{-1 / 2} d_{i}^{\prime} \mathbf{t}_{1}<Z_{i} \leq Q_{0}\left(\lambda_{2}\right)+n^{-1 / 2} d_{i}^{\prime} \mathbf{t}_{2}\right) .
$$

For this reason we see

$$
\begin{aligned}
\left|\delta_{0 n}-\delta_{0}\right|= & c \mid n^{-1} \sum_{i=1}^{n} Z_{1} I\left(Q_{0}\left(\lambda_{1}\right)+n^{-1 / 2} d_{i}^{\prime} \mathbf{t}_{1}<Z_{i} \leq Q_{0}\left(\lambda_{2}\right)+n^{-1 / 2} d_{i}^{\prime} t_{2}\right) \\
& \quad-\int_{Q_{0}\left(\lambda_{1}\right)}^{Q_{0}\left(\lambda_{2}\right)} Z d F_{0}(z) \mid \\
\leq & c\left|n^{-1} \sum_{i=1}^{n} Z_{i} I\left(Z_{i} \leq Q_{0}\left(\lambda_{2}\right)+n^{-1 / 2} d_{i}^{\prime} t_{2}\right)-\nu\left(Q_{0}\left(\lambda_{2}\right)\right)\right| \\
& +c\left|n^{-1} \sum_{i=1}^{n} Z_{i} I\left(Z_{i} \leq Q_{0}\left(\lambda_{1}\right)+n^{-1 / 2} d_{i}^{\prime} t_{1}\right)-\nu\left(Q_{0}\left(\lambda_{1}\right)\right)\right| \\
= & c\left(I_{1}+I_{2}\right)
\end{aligned}
$$


where

$$
\nu(u)=\int_{-\infty}^{u} Z d F_{0}(z)
$$

So, we have to show that $I_{1}=o_{P}(1)=I_{2}$ where

$$
\begin{aligned}
I_{1} \leq & c\left|n^{-1} \sum_{i=1}^{n} Z_{i} I\left(Z_{i} \leq Q_{0}\left(\lambda_{1}\right)+n^{-1 / 2} d_{i}^{\prime} t_{1}\right)-\nu\left(Q_{0}\left(\lambda_{1}\right)+n^{-1 / 2} d_{i}^{\prime} t_{1}\right)\right| \\
& +c\left|\nu\left(Q_{0}\left(\lambda_{1}\right)+n^{-1 / 2} d_{i}^{\prime} t_{1}\right)-\nu\left(Q_{0}\left(\lambda_{1}\right)\right)\right| \\
& =I_{11}+I_{12} .
\end{aligned}
$$

In this case we may write $I_{11}=I_{11}^{+}-I_{11}^{-}$where

$$
I_{11}^{ \pm}=c\left|n^{-1} \sum_{i=1}^{n} Z_{i}^{ \pm} I\left(Z_{i} \leq Q_{0}\left(\lambda_{1}\right)+n^{-1 / 2} d_{i}^{\prime} t_{1}\right)-\nu^{ \pm}\left(Q_{0}\left(\lambda_{1}\right)+n^{-1 / 2} d_{i}^{\prime} \mathbf{t}_{1}\right)\right| .
$$

Then Lemma 2.4 .9 implies that $I_{11}^{ \pm}=o_{P}(1)$. Also, by continuity of $\nu(\cdot)$ we have $I_{12}=o(1)$. Therefore, $I_{1}=o_{P}(1)$ and similarly $I_{2}=o_{P}(1)$.

By similar argument we have

$$
\begin{aligned}
\mid n^{-1} \sum_{i=1}^{n} Z_{i}^{2} I\left(Q_{0}\left(\lambda_{1}\right)+n^{-1 / 2} d_{1}^{\prime} \mathbf{t}_{1}<\right. & \left.Z_{\mathrm{i}} \leq Q_{0}\left(\lambda_{2}\right)+n^{-1 / 2} d_{i}^{\prime} \mathrm{t}_{2}\right) \\
& -\int_{Q_{0}\left(\lambda_{1}\right)}^{Q_{0}\left(\lambda_{2}\right)} Z^{2} d F_{0}(z) \mid=o_{P}(1) .
\end{aligned}
$$

Also, by same argument as (2.147) we have

$$
Q_{\text {or }}(\lambda) \stackrel{P}{\rightarrow} Q_{0}(\lambda) \text {. }
$$

Combining the above results we obtain the proof of Theorem 3.4.9.

\subsection{Trimmed Estimation of Regression Parame- ters Under U'ncertain Prior Information.}

In this section we discuss the estimation of $\boldsymbol{\beta}$ when there exists some uncertain prior information on $\boldsymbol{\beta}$ in the form of a hypothesis. Consider the usual tisear model 
(3.65). We are interested in the robust estimation of $\beta$ when it is suspected but not sure that the null hypothesis

$$
H_{0}: \mathbf{R} \boldsymbol{\beta}=\mathbf{r}
$$

may hold, where $\mathbf{R}$ is a $q \times p+1$ matrix of rank $q(\leq p+1)$. Saleh and Han (1990) considered the problem based on least-squares estimator of $\beta$ while Saleh and Shiraishi (1989) dealt with $R$ and $M$ estimators. In this chapter, we consider $\left(\lambda_{1}, \lambda_{2}\right)$-trimmed estimator for the linear model (3.65) as discussed in section 3.4 and the modification there of. For the model (3.65), we denote the $\left(\lambda_{1}, \lambda_{2}\right)$-trimmed estimator of $\boldsymbol{\beta}$ by $\bar{\beta}_{n}\left(\lambda_{1}, \lambda_{2}\right)$ and is called the unrestricted trimmed L-estimator (UTLE) after trimming off $\lambda_{1}$ and $\lambda_{2}$ proportion of observations using (3.68). When $H_{0}$ is true, the corresponding trimmed estimator of $\beta$ is denoted by $\tilde{\beta}_{n}\left(\lambda_{1}, \lambda_{2}\right)$ and is called the restricted trimmed L-estimator (RTLE). It may be pointed out that $\tilde{\beta}_{n}\left(\lambda_{1}, \lambda_{2}\right)$ has smaller asymptotic dispersion than $\bar{\beta}_{n}\left(\lambda_{1}, \lambda_{2}\right)$ under $H_{0}: \mathbf{R} \boldsymbol{\beta}=\mathbf{r}$, although, $\dot{\beta}_{n}\left(\lambda_{1}, \lambda_{2}\right)$ may be biased and even inconsistent. However, when $H_{0}$ is uncertain, we consider a preliminary test $(\mathrm{PT})$ on $H_{0}$ and consider $\tilde{\beta}_{n}\left(\lambda_{1}, \lambda_{2}\right)$ or $\bar{\beta}_{n}\left(\lambda_{1}, \lambda_{2}\right)$ according as $H_{0}$ is accepted or rejected using a reasonable test statistic. We denote this estimator by $\tilde{\boldsymbol{\beta}}_{n}^{P T}\left(\lambda_{1}, \lambda_{2}\right)$ and is called the preliminary test trimmed L-estimator (PTTLE). Further, we consider some Stein-type estimators incorporating $\bar{\beta}_{n}\left(\lambda_{1}, \lambda_{2}\right)$, $\tilde{\beta}_{n}\left(\lambda_{1}, \lambda_{2}\right)$ and the test statistic. The usual shrinkage trimmed $L$-estimator (STLE) will be denoted by $\tilde{\beta}_{n}^{S}\left(\lambda_{1}, \lambda_{2}\right)$ and the positive-rule shrinkage trimmed L-estimator (PRSTLE) denoted by $\dot{\boldsymbol{\beta}}_{n}^{S+}\left(\lambda_{1}, \dot{\lambda}_{2}\right)$.

In subsection 3.5.1, five trimmed estimators of $\beta$ are introduced. In subsection 3.5.2, the asymptotic distributional quadratic risks(ADQR) of these estimators under local alternatives are investigated. In subsection 3.5.3, the main results on relative dominance of these trimmed estimators are obtained. 


\subsubsection{Five Trimmed Estimators}

This subsection contains the proposed five estimators of $\beta$ stemming out of the TLSE of $\beta$. In the Theorem 3.4.6 (ii), we showed that

$$
\sqrt{n}\left(\bar{\beta}_{n}\left(\lambda_{1}, \lambda_{2}\right)-\beta\right) \stackrel{D}{\longrightarrow} N_{p}\left(0, \sigma^{2}\left(\lambda_{1}, \lambda_{2}\right) \Sigma^{-1}\right)
$$

Now, by analogy of LSE of $\beta$ subject to $R \boldsymbol{\beta}=r$, we propose the RTLE as

$$
\tilde{\boldsymbol{\beta}}_{n}\left(\lambda_{1}, \lambda_{2}\right)=\overline{\boldsymbol{\beta}}_{n}\left(\lambda_{1}, \lambda_{2}\right)-\boldsymbol{\Sigma}_{n}^{-1} \mathbf{R}^{\prime}\left(\mathbf{R} \boldsymbol{\Sigma}_{n}^{-1} \mathbf{R}^{\prime}\right)^{-1}\left(\mathbf{R} \overline{\boldsymbol{\beta}}_{n}\left(\lambda_{1}, \lambda_{2}\right)-\mathbf{r}\right)
$$

To obtain the PTTLE of $\beta$, we consider the following test statistic, $L_{n}$ given by

$$
L_{n}=\frac{\left[\mathbf{R} \bar{\beta}_{n}\left(\lambda_{1}, \lambda_{2}\right)-\mathbf{r}\right]^{\prime}\left[\mathbf{R} \Sigma_{n}^{-1} \mathbf{R}^{\prime}\right]^{-1}\left[\mathbf{R} \bar{\beta}_{n}\left(\lambda_{1}, \lambda_{2}\right)-\mathbf{r}\right]}{q S_{n}^{2}\left(\lambda_{1}, \lambda_{3}\right)}
$$

where $S_{n}^{2}\left(\lambda_{1}, \lambda_{2}\right)$ given in the (3.167). By Theorem 3.4.9, $S_{n}^{2}\left(\lambda_{1}, \lambda_{2}\right) \stackrel{P}{\longrightarrow} \sigma^{2}\left(\lambda_{1}, \lambda_{2}\right)$. Hence, as $n \rightarrow \infty, L_{n}$ converges in law to the central chi-squared distribution with $q$ degrees of freedom $(\dot{d f})$ under $\boldsymbol{H}_{0}$. Hence, we define the PTTLE as

$$
\tilde{\boldsymbol{\beta}}_{n}^{P T}\left(\lambda_{1}, \lambda_{2}\right)=\overline{\boldsymbol{\beta}}_{n}\left(\lambda_{1}, \lambda_{2}\right)-\Sigma_{n}^{-1} \mathbf{R}^{\prime}\left(\mathbf{R} \Sigma_{n}^{-1} \mathbf{R}^{\prime}\right)^{-1}\left(\mathbf{R} \bar{\beta}_{n}\left(\lambda_{1}, \lambda_{2}\right)-\mathbf{r}\right) I\left(L_{n} \leq \lambda_{q, 0}^{2}\right)
$$

where $\chi_{q, \alpha}^{2}$ is the upper $100 \alpha \%$ point of the central chi-square distribution with $q d f$ and $I(A j$ is the indicator function of set $A$. As for the STLE and PRSTLE we propose the following:

$$
\tilde{\boldsymbol{\beta}}_{n}^{S}\left(\lambda_{1}, \lambda_{2}\right)=\overline{\boldsymbol{\beta}}_{n}\left(\lambda_{1}, \lambda_{2}\right)-c L_{n}^{-1} \boldsymbol{\Sigma}_{n}^{-1} \mathbf{R}^{\prime}\left(\mathbf{R} \boldsymbol{\Sigma}_{n}^{-1} \mathbf{R}^{\prime}\right)^{-1}\left(\mathbf{R} \overline{\boldsymbol{\beta}}_{n}\left(\lambda_{1}, \lambda_{2}\right)-\mathbf{r}\right)
$$

and

$$
\tilde{\beta}_{n}^{S+}\left(\lambda_{1}, \lambda_{2}\right)=\tilde{\beta}_{n}\left(\lambda_{1}, \lambda_{2}\right)+\left[1-c L_{n}^{-1}\right] I\left(L_{n}>c\right)\left(\bar{\beta}_{n}\left(\lambda_{1}, \lambda_{2}\right)-\tilde{\beta}_{n}\left(\lambda_{1}, \lambda_{2}\right)\right)
$$

respectively where $0<c<2(q-2)$. Note that as $L_{n} \rightarrow \infty, \tilde{\beta}_{n}^{S}\left(\lambda_{1}, \lambda_{2}\right)\left\{\tilde{\beta}_{n}^{S+}\left(\lambda_{1}, \lambda_{2}\right)\right\} \rightarrow$ $\overline{\boldsymbol{\beta}}_{n}\left(\lambda_{1}, \lambda_{2}\right)$ and as $L_{n} \rightarrow c, \tilde{\beta}_{n}^{S}\left(\lambda_{1}, \lambda_{2}\right)\left\{\tilde{\beta}_{n}^{S+}\left(\lambda_{1}, \lambda_{2}\right)\right\} \rightarrow \tilde{\beta}_{n}\left(\lambda_{1}, \lambda_{2}\right)$, which is similar to the property of PTTLE but (3.185) and (3.186) are smooth version of PTTLE. 


\subsubsection{Asymptotic Distributional Risks}

We consider the asymptotic distributional risks under local alternatives, noting that the test based on $L_{n}$ is consistent for fixed $\beta$ such that $R \beta \neq r$ and the PTTLE, STLE and PRSTLE are asymptotically equivalent to UTLE under fixed alternatives. Hence, we study the asymptotic risks under local alternatives:

$$
\mathbf{H}_{\mathbf{a}}: \mathbf{R} \boldsymbol{\beta}=\mathbf{r}+n^{-\frac{1}{2} \boldsymbol{\delta}}, \quad \boldsymbol{\delta}=\left(\delta_{1}, \cdots, \delta_{q}\right)^{\prime} .
$$

Then we have the following theorem.

Theorem 3.5.1 Under $\left\{H_{a}\right\}$ and conditions of theorem 3.4.6, the distribution of $L_{n}, \bar{\beta}_{n}\left(\lambda_{1}, \lambda_{2}\right), \tilde{\beta}_{n}\left(\lambda_{1}, \lambda_{2}\right)$, and $\tilde{\beta}_{n}^{P T}\left(\lambda_{1}, \lambda_{2}\right)$ are given by

(i) $\lim P\left\{L_{n} \leq \mathbf{x}\right\}=H_{q}(\mathbf{x}, \Delta), \quad \Delta=\frac{\boldsymbol{\delta}^{\prime}\left(\mathbf{R} \Sigma^{-1} \mathbf{R}^{\prime}\right)^{-1} \boldsymbol{\delta}}{\sigma^{2}\left(\lambda_{1}, \lambda_{2}\right)}$

(ii) $\lim P\left\{\sqrt{n}\left(\overline{\boldsymbol{\beta}}_{n}\left(\lambda_{1}, \lambda_{2}\right)-\boldsymbol{\beta}\right) \leq \mathbf{x}\right\}=G_{p+1}\left(\mathbf{x} ; \mathbf{0}, \sigma^{2}\left(\lambda_{1}, \lambda_{2}\right) \Sigma^{-1}\right)$

(iii) $\lim P\left\{\sqrt{n}\left(\tilde{\beta}_{n}\left(\lambda_{1}, \lambda_{2}\right)-\beta\right) \leq \mathbf{x}\right\}$

$$
=G_{p+1}\left(\mathbf{x}+\Sigma^{-1} \mathbf{R}^{\prime}\left(\mathbf{R} \Sigma^{-1} \mathbf{R}^{\prime}\right)^{-1} \boldsymbol{\delta} ; \mathbf{0}, \sigma^{2}\left(\lambda_{1}, \lambda_{2}\right) \mathbf{V}\right)
$$

(iv) $\lim P\left\{\sqrt{n}\left(\dot{\boldsymbol{\beta}}_{n}^{P T}\left(\lambda_{1}, \lambda_{2}\right)-\boldsymbol{\beta}\right) \leq \mathbf{x}\right\}=$

$$
\begin{gathered}
H_{\imath}\left(\chi_{q, \alpha}^{2} ; \Delta\right) G_{p+1}\left\{\mathbf{x}+\Sigma^{-1} \mathbf{R}^{\prime}\left(\mathbf{R} \Sigma^{-1} \mathbf{R}^{\prime}\right)^{-1} \delta ; 0, \sigma^{2}\left(\lambda_{1}, \lambda_{2}\right) \mathbf{V}\right\} \\
+\int_{E(\delta)} G_{p+1}\left\{\mathbf{x}-\Sigma^{-1} \mathbf{R}^{\prime}\left(\mathbf{R} \Sigma^{-1} \mathbf{R}^{\prime}\right)^{-1} z ; 0, \sigma^{2}\left(\lambda_{1}, \lambda_{2}\right) \mathbf{V}\right\} \\
d G_{p+1}\left(z ; 0, \sigma^{2}\left(\lambda_{1}, \lambda_{2}\right)\left(\mathbf{R} \Sigma^{-1} \mathbf{R}^{\prime}\right)\right)
\end{gathered}
$$

where

$$
\mathbf{V}=\boldsymbol{\Sigma}^{-1}-\boldsymbol{\Sigma}^{-1} \mathbf{R}^{\prime}\left(\mathbf{R} \boldsymbol{\Sigma}^{-1} \mathbf{R}^{\prime}\right)^{-1} \mathbf{R} \boldsymbol{\Sigma}^{-1}
$$

and $H_{q}(\cdot ; \Delta)$ is the cdf of a non-central chi-square distribution with $q$ df and noncentrality parameter $\Delta, G_{p+1}(\mathbf{x} ; \mu, \mathbf{\Sigma})$ is the cdf of a $p+1$-dimensional normal 
distribution with mean $\mu$ and covariance matrix $\Sigma$ and

$$
E(\delta)=\left\{z: \frac{(z+\delta)^{\prime}\left(R \Sigma^{-1} R^{\prime}\right)^{-1}(z+\delta)}{\sigma^{2}\left(\lambda_{1}, \lambda_{2}\right)} \geq \chi_{r, a}^{2}\right\}
$$

Furthermore,

$$
\begin{aligned}
& \text { (v) } \quad \sqrt{n}\left(\tilde{\beta}_{n}^{S}\left(\lambda_{1}, \lambda_{2}\right)-\beta\right) \stackrel{D}{=} \\
& \mathbf{U}-c \sigma^{2}\left(\lambda_{1}, \lambda_{2}\right) \frac{\Sigma^{-1} \mathbf{R}^{\prime}\left(\mathbf{R} \Sigma^{-1} \mathbf{R}^{\prime}\right)^{-1}(\mathbf{R U}+\delta)}{(\mathbf{R U}+\delta)^{\prime}\left(\mathbf{R} \Sigma^{-1} \mathbf{R}^{\prime}\right)^{-1}(\mathbf{R U}+\delta)}
\end{aligned}
$$

where

$$
\mathbf{U} \stackrel{\mathcal{D}}{=} \mathcal{N}_{p+1}\left(0, \sigma^{2}\left(\lambda_{1}, \lambda_{2}\right) \Sigma^{-1}\right)
$$

Similarly,

$$
\text { (vi) } \quad \begin{gathered}
\sqrt{n}\left(\bar{\beta}_{n}^{s+}\left(\lambda_{1}, \lambda_{2}\right)-\beta\right) \stackrel{D}{=} \mathbf{U}-\Sigma^{-1} \mathbf{R}^{\prime}\left(\mathbf{R} \Sigma^{-1} \mathbf{R}^{\prime}\right)^{-1}(\mathbf{R U}+\delta) \\
+\left\{1-c \sigma^{2}\left(\lambda_{1}, \lambda_{2}\right)\left[(\mathbf{R U}+\delta)^{\prime}\left(\mathbf{R} \Sigma^{-1} \mathbf{R}^{\prime}\right)^{-1}(\mathbf{R U}+\delta)\right]^{-1}\right\} \\
I\left\{(\mathbf{R U}+\delta)^{\prime}\left(\mathbf{R} \Sigma^{-1} \mathbf{R}^{\prime}\right)^{-1}(\mathbf{R U}+\delta) \geq c\right\} \\
\Sigma^{-1} \mathbf{R}^{\prime}\left(\mathbf{R} \Sigma^{-1} \mathbf{R}^{\prime}\right)^{-1}(\mathbf{R U}+\delta)
\end{gathered}
$$

Assume that, for an estimator $\tilde{\beta}_{n}^{*}\left(\lambda_{1}, \lambda_{2}\right)$ of $\beta$,

$$
G^{*}(\mathbf{x})=\lim P\left\{\sqrt{n}\left(\tilde{\beta}_{n}^{*}\left(\lambda_{1}, \lambda_{2}\right)-\beta\right) \leq x\right\}
$$

Then, we define the asymptotic distributional quadratic risk (ADQR) of $\grave{\beta}_{n}^{*}\left(\lambda_{1}, \lambda_{2}\right)$ by

$$
\Re\left(\tilde{\beta}_{n}^{*}\left(\lambda_{1}, \lambda_{2}\right) ; \mathbf{W}\right)=\int_{R^{p}} \mathbf{x}^{\prime} \mathbf{W} \mathbf{x} d G^{*}(\mathbf{x})=\operatorname{tr}\left(\Sigma^{*} \mathbf{W}\right)
$$

where

$$
\boldsymbol{\Sigma}^{*}=\int \mathbf{x x}^{\prime} d G^{*}(\mathbf{x})
$$

and $\mathbf{W}$ is a positive definite matrix associated with the quadratic loss function

$$
L\left(\tilde{\beta}_{n}^{*}\left(\lambda_{1}, \dot{\lambda}_{2}\right) ; \beta\right)=n\left(\tilde{\beta}_{n}^{*}\left(\lambda_{1}, \lambda_{2}\right)-\beta\right)^{\prime} \mathbf{W}\left(\tilde{\beta}_{n}^{*}\left(\lambda_{1}, \lambda_{2}\right)-\beta\right)
$$


Theorem 3.5.2 Under the assumption of theorem 9.5.1, the ADQR's of the estimators are given by

(i) $\Re\left(\bar{\beta}_{n}\left(\lambda_{1}, \lambda_{2}\right) ; W\right)=\sigma^{2}\left(\lambda_{1}, \lambda_{2}\right) \operatorname{tr} \Sigma^{-1} \mathbf{W}$,

(ii) $\Re\left(\tilde{\beta}_{n}\left(\lambda_{1}, \lambda_{2}\right) ; W\right)=\sigma^{2}\left(\lambda_{1}, \lambda_{2}\right)\left[\operatorname{tr}\left(\Sigma^{-1} \mathbf{W}\right)-\operatorname{tr}(\mathbf{B})\right]+\sigma^{\prime}\left(\mathbf{R} \Sigma^{-1} \mathbf{R}^{\prime}\right)^{-1} \mathbf{B} \delta$

(iii) $\quad \Re\left(\bar{\beta}_{n}^{P T}\left(\lambda_{1}, \lambda_{2}\right) ; W\right)=\sigma^{2}\left(\lambda_{1}, \lambda_{2}\right) \operatorname{tr}\left(\Sigma^{-1} \mathbf{W}\right)-\sigma^{2}\left(\lambda_{1}, \lambda_{2}\right) \operatorname{tr}(B) H_{q+2}\left(\chi_{q, a}^{2} ; \Delta\right)$

$$
+\delta^{\prime}\left(\mathbf{R} \Sigma^{-1} \mathbf{R}^{\prime}\right)^{-1} \mathrm{~B} \delta\left\{2 H_{q+2}\left(\chi_{q, \alpha}^{2} ; \Delta\right)-H_{q+1}\left(x_{q, a}^{2} ; \Delta\right)\right\} \text {, }
$$

(iv) $\quad \Re\left(\hat{\beta}_{n}^{S}\left(\lambda_{1}, \lambda_{2}\right) ; \mathbf{W}\right)=\sigma^{2}\left(\lambda_{1}, \lambda_{2}\right) \operatorname{tr}\left(\Sigma^{-1} \mathbf{W}\right)-\sigma^{2}\left(\lambda_{1}, \lambda_{2}\right) c \operatorname{tr}(\mathbf{B})\left\{2 E\left[\lambda_{q+2}^{-2}(\Delta)\right]\right.$

$$
\left.-c E\left[\chi_{q+2}^{-4}(\Delta)\right]\right\}+c(c+4)\left\{\sigma^{\prime}\left(\mathbf{R} \Sigma^{-1} \mathbf{R}^{\prime}\right)^{-1} \mathbf{B} \delta E\left[\chi_{q+4}^{-4}(\Delta)\right]\right\},
$$

(v) $\quad \Re\left(\tilde{\beta}_{n}^{S+}\left(\dot{\lambda}_{1}, \lambda_{2}\right) ; \mathbf{W}\right)=\Re\left(\dot{\boldsymbol{\beta}}_{n}^{S} ; \mathbf{W}\right)-\sigma^{2}\left(\lambda_{1}, \lambda_{2}\right) \operatorname{tr}(\mathbf{B})$

$$
\begin{gathered}
E\left[\left(1-c \chi_{q+2}^{-2}(\Delta)\right)^{2} I\left(\chi_{q+2}^{2}(\Delta) \leq c\right)\right] \\
+\delta^{\prime}\left(\mathbf{R} \Sigma^{-1} \mathbf{R}\right)^{-1} \mathbf{B} \delta\left\{2 E\left[\left(1-c \lambda_{q+2}^{-2}(\Delta)\right) I\left(\lambda_{q+2}^{2} \leq c\right)\right]\right. \\
\left.-E\left[\left(1-c \lambda_{q+4}^{-2}(\Delta)\right) I\left(\lambda_{q+4}^{2}(\Delta) \leq c\right)\right]\right\}
\end{gathered}
$$

where

$$
\mathbf{B}=\mathbf{R} \boldsymbol{\Sigma}^{-1} \mathbf{W} \boldsymbol{\Sigma}^{-1} \mathbf{R}^{\prime}\left(\mathbf{R} \boldsymbol{\Sigma}^{-1} \mathbf{R}\right)^{-1}
$$

\section{Proof:}

(i) and (ii) follow by straight forward computations using Theorem 3.5.1 (ii) and (iii). Using Theorem 3.5.1 (iv) - (v), (iii), (iv) and (v) are given by the same argument as in Judge and Bock (1978).

\subsubsection{Comparison of ADQR}

First, we compare the $\bar{\beta}_{n}\left(\lambda_{1}, \lambda_{2}\right)$ with $\bar{\beta}_{n}\left(\lambda_{1}, \lambda_{2}\right)$ by the ratio of the ADQR of 
$\bar{\beta}_{n}\left(\lambda_{1}, \lambda_{2}\right)$ to that of $\bar{\beta}_{n}\left(\lambda_{1}, \lambda_{2}\right)$.

$$
\operatorname{ARE}\left[\bar{\beta}_{n}\left(\lambda_{1}, \lambda_{2}\right)_{i} \bar{\beta}_{n}\left(\lambda_{1}, \lambda_{2}\right)\right]=\left\{1-\frac{\operatorname{tr}(\mathbf{B})}{\operatorname{tr}\left(\Sigma^{-1} \mathbf{W}\right)}+\frac{\delta^{\prime}\left(\mathbf{R} \Sigma^{-1} \mathbf{R}^{\prime}\right)^{-1} \mathbf{B} \delta}{\sigma^{2}\left(\lambda_{1}, \lambda_{2}\right) \operatorname{tr}\left(\Sigma^{-1} \mathbf{W}\right)}\right\}^{-1} .
$$

Hence, $A R E\left[\tilde{\beta}_{n}\left(\lambda_{1}, \lambda_{2}\right) ; \tilde{\beta}_{n}\left(\lambda_{1}, \lambda_{2}\right)\right] \stackrel{\leq}{1}$ according as

$$
\sigma^{\prime}\left(\mathbf{R} \Sigma^{-1} \mathbf{R}^{\prime}\right)^{-1} \mathbf{B} \delta \leq \sigma^{2}\left(\lambda_{1}, \lambda_{2}\right) \operatorname{tr}\left(\Sigma^{-1} \mathbf{W}\right) .
$$

Specially, if $\sigma=0$, ie, under $H_{0}, A R E\left(\tilde{\beta}_{n}\left(\lambda_{1}, \lambda_{2}\right) ; \bar{\beta}_{n}\left(\lambda_{1}, \lambda_{2}\right)\right) \geq 1$. Let $c h_{\min }(B)$ and $c h_{\text {max }}(B)$ be the smallest and largest characteristic root of $B$, then using the relation

$$
\begin{gathered}
\operatorname{ch} h_{\min }(\mathbf{B}) \Delta \leq \frac{\delta^{\prime}\left(\mathbf{R} \Sigma^{-1} \mathbf{R}^{\prime}\right)^{-1} \mathbf{B} \delta}{\sigma^{2}\left(\lambda_{1}, \lambda_{2}\right)} \leq c h_{\max }(\mathbf{B}) \Delta \\
\left\{1-\frac{\operatorname{tr}(\mathbf{B})}{\operatorname{tr}\left(\Sigma^{-1} \mathbf{W}\right)}+\frac{\operatorname{ch} \max (\mathbf{B}) \Delta}{\operatorname{tr}\left(\Sigma^{-1} \mathbf{W}\right)}\right\}^{-1} \leq A R E\left(\bar{\beta}_{n}\left(\lambda_{1}, \lambda_{2}\right), \bar{\beta}_{n}\left(\lambda_{1}, \lambda_{2}\right)\right) \\
\leq\left\{1-\frac{\operatorname{tr}(\mathbf{B})}{\operatorname{tr}\left(\Sigma^{-1} \mathbf{W}\right)}+\frac{\operatorname{ch} \min (\mathbf{B}) \Delta}{\operatorname{tr}\left(\Sigma^{-1} \mathbf{W}\right)}\right\}^{-1}
\end{gathered}
$$

Both sides of inequality (3.20E) are decreasing in $\Delta$. Next, we investigate the ARE of $\tilde{\beta}_{n}^{P T}\left(\lambda_{1}, \lambda_{2}\right)$ relative to $\bar{\beta}_{n}\left(\lambda_{1}, \lambda_{2}\right)$.

$$
\operatorname{ARE}\left(\tilde{\beta}_{n}^{P T}\left(\lambda_{1}, \lambda_{2}\right), \bar{\beta}_{n}\left(\lambda_{1}, \lambda_{2}\right)\right)=[1+h(\delta)]^{-1}
$$

where

$$
\begin{aligned}
h(\delta)=\sigma^{-2}\left(\lambda_{1}, \lambda_{2}\right)\left\{\delta^{\prime}\left(\mathbf{R} \Sigma^{-1} \mathbf{R}^{\prime}\right)^{-1} \mathbf{B} \delta\right\}\left\{\frac{2 H_{q+2}\left(\chi_{q, a}^{2}, \Delta\right)-H_{q+4}\left(\chi_{q, \alpha}^{2}, \Delta\right)}{\operatorname{tr}\left(\Sigma^{-1} \mathbf{W}\right)}\right\} \\
-\left\{\frac{\operatorname{tr}(\mathbf{B}) H_{0+2}\left(\chi_{q, a}^{2} ; \Delta\right)}{\operatorname{tr}\left(\Sigma^{-1} \mathbf{W}\right)}\right\} .
\end{aligned}
$$

Hence we get $A R E\left(\tilde{\beta}_{n}^{P T}\left(\lambda_{1}, \lambda_{2}\right), \bar{\beta}_{n}\left(\lambda_{1}, \lambda_{2}\right)\right) \leqq 1 \quad$ according to

$$
\frac{\delta^{\prime}\left(\mathbf{R} \Sigma^{-1} \mathbf{R}^{\prime}\right)^{-1} \mathbf{B} \delta}{\sigma^{2}\left(\lambda_{1}, \lambda_{2}\right)} \leqq \frac{\operatorname{tr}(\mathbf{B}) H_{q+2}\left(\chi_{q, a}^{2} ; \Delta\right)}{2 H_{q+2}\left(\chi_{q, a}^{2}, \Delta\right)-H_{q+1}\left(\chi_{q, \alpha}^{2}, \Delta\right)}
$$


Also let us define

$$
\begin{aligned}
& h_{\max }(\Delta)=\operatorname{ch}_{\max }(\mathrm{B}) \Delta\left\{\frac{2 H_{0+2}\left(\chi_{p, a,}^{2}, \Delta\right)-H_{1+4}\left(\chi_{q, a}^{2}, \Delta\right)}{\operatorname{tr}\left(\Sigma^{-1} W\right)}\right. \\
& \left.-\frac{\operatorname{tr}(B) H_{a+2}\left(\chi_{a, a}^{2} ; \Delta\right)}{\operatorname{tr}\left(\Sigma^{-1} W\right)}\right\} \\
& h_{\min }(\Delta)=c h_{\min }(\mathrm{B}) \Delta\left\{\frac{2 H_{q+2}\left(\chi_{q, a}^{2}, \Delta\right)-H_{q+1}\left(\chi_{q, a,}^{2}, \Delta\right)}{\operatorname{tr}\left(\Sigma^{-1} \mathbf{W}\right)}\right. \\
& \left.-\frac{\operatorname{tr}(\mathrm{B}) H_{\mathrm{a}+2}\left(\chi_{1,0 ; \Delta}^{2} ; \Delta\right)}{\operatorname{tr}\left(\Sigma^{-1} \mathrm{~W}\right)}\right\}
\end{aligned}
$$

Then we obtain the inequality

$$
\left[1+h_{\max }(\Delta)\right]^{-1} \leq A R E\left(\dot{\beta}_{n}^{P T}\left(\lambda_{1}, \lambda_{2}\right), \bar{\beta}_{n}\left(\lambda_{1}, \lambda_{2}\right)\right) \leq\left[1+h_{\min }(\Delta)\right]^{-1}
$$

We compare $\tilde{\beta}_{n}\left(\lambda_{1}, \lambda_{2}\right)$ with $\bar{\beta}_{n}\left(\lambda_{1}, \lambda_{2}\right)$. The difference of the risks is given by

$$
\begin{aligned}
& \Re\left(\bar{\beta}_{n}\left(\lambda_{1}, \lambda_{2}\right) ; \mathbf{W}\right)-\Re\left(\dot{\beta}_{n}^{S}\left(\lambda_{1}, \lambda_{2}\right) ; \mathbf{W}\right)= \\
& \sigma^{2}\left(\lambda_{1}, \lambda_{2}\right) c \operatorname{tr}(\mathbf{B})\left\{2 E\left[\chi_{q+2}^{-2}(\Delta)\right]-c E\left[\chi_{q+2}^{-4}(\Delta)\right]\right\} \\
& -c(c+4)\left\{\delta^{\prime}\left(\mathbf{R} \Sigma^{-1} \mathbf{R}^{\prime}\right)^{-1} \mathbf{B} \delta E\left[\chi_{q+4}^{-4}(\Delta)\right]\right\} .
\end{aligned}
$$

Using the relation (3.204), the R.H.S of (3.212) is bounded from below by

$$
\begin{aligned}
\sigma^{2}\left(\lambda_{1}, \lambda_{2}\right) c & \operatorname{tr}(\mathbf{B})\left\{2 E\left[\chi_{q+2}^{-2}(\Delta)\right]\right. \\
& \left.-c E\left[\chi_{q+2}^{-4}(\Delta)\right]\right\}-c(c+4) c h_{\max }(\mathbf{B}) \Delta E\left[\chi_{q+4}^{-4}(\Delta)\right]
\end{aligned}
$$

We have also the relation

$$
E\left[\chi_{q+2}^{-2}(\Delta)\right]-(q-2) E\left[\chi_{q+2}^{-4}(\Delta)\right]=\Delta E\left[\chi_{q+4}^{-4}(\Delta)\right]
$$

We may rewrite (3.213) as

$$
\sigma^{2}\left(\lambda_{1}, \lambda_{2}\right) c \operatorname{tr}(\mathbf{B})\left\{(2-g) E\left[\chi_{q+2}^{-2}(\Delta)\right]+\{(q-2) g-c\} E\left[\chi_{q+2}^{-4}(\Delta)\right]\right\}
$$


where $g=(c+4) h$ and $h=c h_{\max }(B) / \operatorname{tr}(B)$. Thus, for (3.212) to be non-negative for all $\delta$ it suffices to choose $c$ such that

$$
0 \leq g \leq 2 \quad \text { and } \quad(q-2) g \geq c \geq 0
$$

Note that $g \leq 2$ is equivalent to $c \leq 2\left(h^{-1}-2\right)$. This implies that $\operatorname{tr}(B)>$ $2 c h_{\max }(B)$ is the minimum requirement for (3.212) to be non-negative. Also, from non-negativeness of (3.215) at $\delta=0$, it follows that $c$ has to be less than or equal to $2(q-2)(\Rightarrow q \geq 3)$. Hence, we get

Theorem 3.5.3 A sufficient condition for the asymptotic dominance of the STLE over UTLE $\left[\right.$ i.e $\Re\left(\bar{\beta}_{n}\left(\lambda_{2}, \lambda_{2}\right) ; W\right) \geq \Re\left(\tilde{\beta}_{n}^{S}\left(\lambda_{1}, \lambda_{2}\right) ; W\right)$ for all $\delta \in R^{\eta}$ and $\left.\mathbf{W}(p . d)\right]$ is that the shrinkage factor $c$ is positive and it satisfies the follouing inequality

$$
2 E\left[\chi_{q+2}^{-2}(\Delta)\right]-c E\left[\chi_{q+2}^{-4}(\Delta)\right]-(c-4) h \Delta E\left[\chi_{p+4}^{-4}(\Delta)\right] \geq 0 \quad \forall \Delta \geq 0
$$

which, in turn, requires that $q \geq 3, \quad 0<c<2(q-2)$

and $\operatorname{tr}(\mathrm{B})>2 \operatorname{ch}_{\max }(\mathrm{B})$.

By the same argument as in the proof of Theorem 4.3 of Sen and Saleh (1987) we get

Theorem 3.5.4 Under the sufficient condition of Theorem 3.5.3 the PTTLE fails to dominate the STLE. Also, if for $\alpha$, the level of significance of the preliminary test, we have

$$
\begin{aligned}
H_{q+2}\left(\lambda_{q, a}^{2} ; 0\right) & \geq r[2(q-2)-r] /[q(q-2)] \\
r & =(q-2) \wedge[2 /(h-4)]
\end{aligned}
$$

then STLE fails to dominate the PTTLE.

Finally, we compare $\tilde{\beta}_{n}^{S+}\left(\lambda_{1}, \lambda_{2}\right)$ with $\tilde{\beta}_{n}^{S}\left(\lambda_{1}, \lambda_{2}\right)$, the difference of the risks is given by

$$
\mathfrak{R}\left(\tilde{\boldsymbol{\beta}}_{n}^{\mathcal{S}+}\left(\lambda_{1}, \lambda_{2}\right) ; \mathbf{W}\right)-\mathfrak{R}\left(\tilde{\boldsymbol{\beta}}_{n}^{S}\left(\lambda_{1}, \lambda_{2}\right) ; \mathbf{W}\right)=
$$




$$
\begin{aligned}
& -\left\{\sigma^{2}\left(\lambda_{1}, \lambda_{2}\right) \operatorname{tr}(\mathbf{B}) E\left[\left(1-c \chi_{q+2}^{-2}(\Delta)\right)^{2} I\left(\chi_{q+2}^{2}(\Delta) \leq c\right)\right]\right. \\
& +\delta^{\prime}\left(\mathbf{R} \Sigma^{-1} \mathbf{R}\right)^{-1} \mathbf{B} \delta\left(E\left[\left(1-c \chi_{q+4}^{-2}(\Delta)\right) I\left(\chi_{q+4}^{2}(\Delta) \leq c\right)\right]\right. \\
& \left.\left.-2 E\left[\left(1-c \chi_{q+2}^{-2}(\Delta)\right) I\left(\chi_{q+2}^{2} \leq c\right)\right]\right)\right\} .
\end{aligned}
$$

By using the relation

$$
E\left[\chi_{p+2}^{-2}(\Delta)\right]-E\left[\chi_{p+4}^{-2}(\Delta)\right]=2 E\left[\chi_{p+2}^{-4}(\Delta)\right]
$$

The R.H.S of (3.215) is always negative so

\section{Theorem 3.5.5 PSTLE dominates STLE.}

In general the dominance picture may be stated as

$$
\Re\left(\dot{\boldsymbol{\beta}}_{n}\left(\lambda_{1}, \lambda_{2}\right): \mathbf{W}\right)<\Re\left(\dot{\boldsymbol{\beta}}_{n}^{S+}\left(\lambda_{1}, \lambda_{2}\right): \mathbf{W}\right)<\Re\left(\dot{\boldsymbol{\beta}}_{n}^{S}\left(\lambda_{1}, \lambda_{2}\right): \mathbf{W}\right)<\Re\left(\dot{\beta}_{1}\left(\lambda_{1}, \lambda_{2}\right): \mathbf{W}\right)
$$

while $\Re\left(\dot{\boldsymbol{\beta}}_{n}^{P T}\left(\lambda_{1}, \lambda_{2}\right)\right.$; W) may sit between $\Re\left(\dot{\boldsymbol{\beta}}_{n}\left(\lambda_{1}, \lambda_{2}\right): \mathbf{W}\right)$ and $\Re\left(\dot{\beta}_{n}\left(\lambda_{1}, \lambda_{2}\right): \mathbf{W}\right)$ depending on the size of the test.

\subsubsection{Conclusion}

In this section, we proposed several alternative estimators of the regression parameters based on trimmed least squares in the linear model. It is observed that the performance of the preliminary test estimators near tie nu!!-hypothesis is generally better than other estimators depending on the size of the test wilile the shrinkage estimators having uniform dominance and never depend on the size of the t.ast. as such are preferable. The positive rui is preferred over the usual shrinkage estimator. However, we notice that the shrinkage stimator depends on the rank of the matrix $\mathbb{R}$ which is $q$ and the requirement for the application is thit $q \geq 3$ while the 

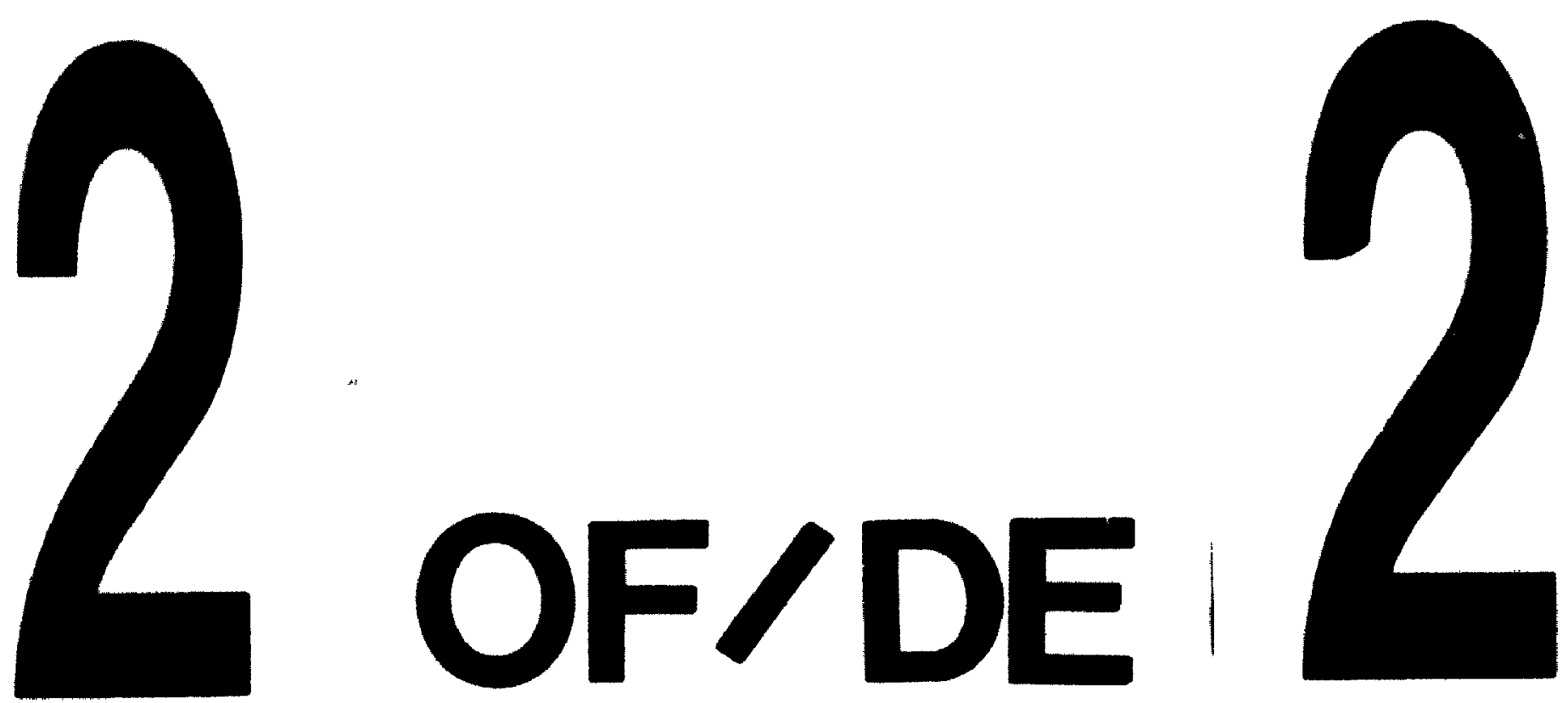

PM-1 3\%" "X4" PHOTOCRAPMIC MICROCOPY TARCET NAS $1010 a$ ANS1/1SO *2 EOUIVALENT

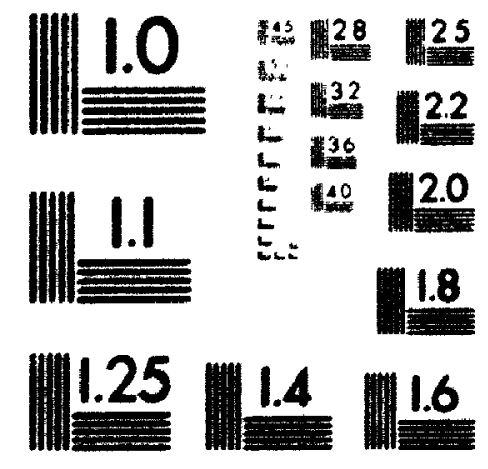

PRECISLNAM RESOLUTION TARETT 
preliminary test estimator does not depend on the size of q. Thus for y $\leq \geq$, one is forced to use the preliminary +rst estimator. For optinum choice of the size of $\alpha$, one may benefit using preliminary test estimator while shrinkage estimators are always preferred provided $q \geq 3$.

the following graphs represent the risk of the five estimators discussed in this section. .

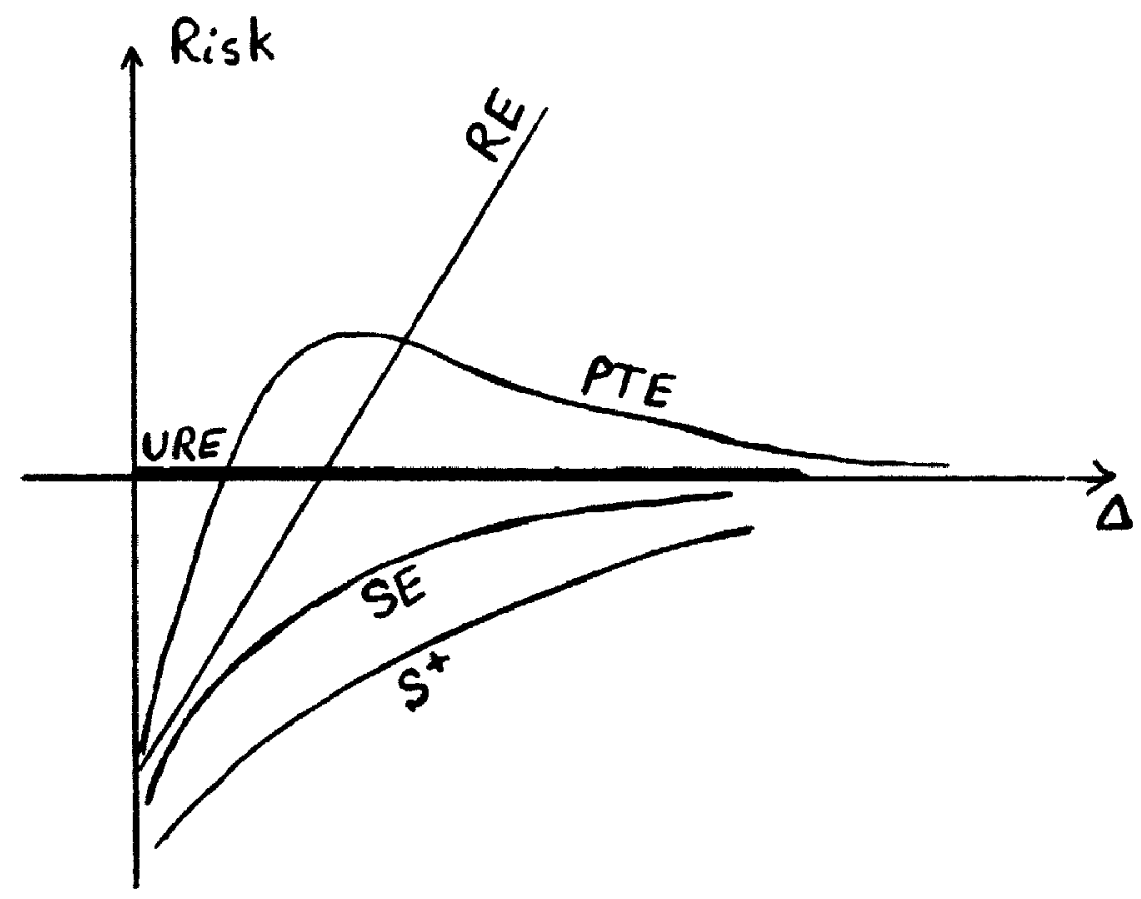

Figure 3.2: Risk of the five estimators 


\section{Chapter 4}

\section{Autoregression Model}

\subsection{Introduction}

The purpose of this chapter is to present the autoregression quantile (ARQ) approach to some aspects of $L$ - estimation of the parameters for the $p^{\text {th }}$ order autoregression. AR( $\boldsymbol{p})$-models. This approach is similar to the previous chapter in commection with the linear models and thereby extends the statistical nethodology to the most applied models with dependent observations. For the AR(p)-models with Gaussian errors, Saleh (\$992) discusses various improved estimators of the parameters based on maximum likelihood estimators (MLEs) and Koul and Salel (1993) considers the rank theory version of the same problem when the errors are i.i.d r.v's from some unknown $d . f F_{0}$. In this chapter, we are particularly concerned with : extension of the idea of L-estimation based on a few selected $A R Q ' s$ and the trimmed least squarcs estimators in the linear model to the AR(p)-models using the ideas of hoenker and Bassett (1978) and Jurečková (1984) for the linear models. Though the results are parallel, the analysis is complex to put the matter in rigorous terms.

The material of this chapter is presented in three sections. Section 4.2 contains the introduction to the $A R(p)$-model along with its basic properties and the least 
squares estimator of the parameters. It also contains the definition of autoregres. sion quantile (ARQ) introduced by houl and Saleh (1995). The basic ideat of the developments for the AR(p) model on autoregression quantile's (ARQ) are based on randomly weighted empirical process due to houl and Ossainder (1991). Here, we present the asymptotic properties and the asymptotic representation of AIRQ $\mathrm{s}$. Section 4.3 contains the discussion of L-estimation of parameters of an auturegression model based on a few selected autoregression quantiles. In section 4.4 we present the trimmed least squares estimators of the autoregression paranueters while ser tion 4.5 contains estimation of the autoregression parameters under uncertain prior information.

\subsection{Autoregression Quantiles}

Consider the autoregression model

$$
X_{1}=\rho_{0}+\rho_{1} X_{2-1}+\cdots+\rho_{p} X_{i-p}+Z_{2} \quad i=0, \pm 1, \pm:, \cdots
$$

where $p(\geq 1)$ is a fixed integer, $\rho:=\left(\rho_{0}, \rho_{1}, \cdots, \rho_{r}\right)^{\prime} \in \mathbb{E}^{p+1}$ is the vector of pai rameters in (4.1) belonging to the $p+1$ dinensional Euclidean space, and $Z_{i}^{\prime}, i:-$ $0, \pm 1, \pm 2, \cdots$ are $\mathrm{i} \cdot \mathrm{i} \cdot \mathrm{d}$ random variables with continuous distribution function triv. We assume further the following:

$$
\mathrm{E}\left(Z_{i}\right)=0 \quad \mathrm{E}\left(Z_{i}^{2}\right)<\infty
$$

a2) all roots of the equation $x^{p}-\rho_{1} x^{p-1}-\cdots-\rho_{p}=0$ are inside 1 he untit circle for all $x \in C$.

For a given sample, let us rewrite the model (4.1) in the form

$$
X_{\mathbf{1}}=\boldsymbol{X}_{\mathrm{i}-1}^{\prime} \rho+Z_{1}, \quad i=0, \pm 1, \pm 2, \cdots
$$

where $\boldsymbol{X}_{1-1}=\left(1, X_{i-1}, \cdots, X_{1-p}\right)^{\prime}$ and let $\boldsymbol{X}_{n}$ be the $n,(p+1)$ design matrix with the $i^{\text {th }}$ row being $X_{1-1}^{\prime},(1 \leq i \leq n)$. Since the first clenent of $X_{1-1}$ in cone, 
wr define the components of $\boldsymbol{X}_{i-1}$ as a $\boldsymbol{X}_{i-j}, j=0,1, \ldots, p$. In the analysis of the autoregression model, under the conditions $a 1$ and $a 2$, the AR(p) model is stationary, causal and invertible. Details may be found in Brockwell and Davis (1991). The invertibility property of $\left\{X_{1}\right\}$ together with continuity of $F_{0}$ implies that the rows and columns of $\mathcal{X}_{n}$ are linearly independent w.p.1. This allows us to consider the use of the inverse of $\mathcal{X}_{n}$ in the sequel. The causal property with Theorem IV.2.3 of Hannan (1970) implies that the process $\left\{X_{1}\right\}$ is ergodic. We need the ergodic property to prove some of the Theorems in this chapter. The following conditions of the design matrix $\mathcal{X}_{n}$ are implied by assumption $a 1$ and $a$ ?.

$$
n^{-1 / 2} \max _{i}\left|X_{i-1}\right|=o_{P}(1) \text {. }
$$

B:2 $\left\{X_{i, j}, 1 \leq i \leq n\right\}$ is square integrable for each $n \geq 1$.

B33

$$
n^{-1} \mathcal{X}_{n}^{\prime} \mathcal{X}_{n}=\Sigma_{n} \stackrel{P}{\rightarrow} \boldsymbol{\Sigma} \quad p \cdot d .
$$

Before stating the results we introduce the necessary definitions and assumptions as follows:

Let $(\Omega, \mathcal{F}, P)$ be a probability space and $\left(Z_{i}, X_{i-}, \xi_{n i}\right), 1 \leq i \leq n$ be ant array of trivariate r.v's defined on $(\Omega, \mathcal{F})$ such that $\left\{Z_{i}\right\}$ are i.i.d. $F_{0}$ and $Z_{i}$ is independent of $\left(X_{1-j}, \xi_{n t}\right), 1 \leq i \leq n$. Furthermore. let $\left\{\mathcal{F}_{1}\right\}$ be an array of suboficlds such that $\mathcal{F}_{1} \subset \mathcal{F}_{1+1}, 1 \leq i \leq n ;\left(X_{1-\jmath}, \xi_{n 1}\right)$ is $\mathcal{F}_{1}$-measurable, the $r \cdot v$ 's $\left\{Z_{1}, \cdots, Z_{l-1} ;\left(X_{1-j}, \xi_{n i}\right), 1 \leq i \leq l\right\}$ are $\mathcal{F}_{l}$-measurable, $2 \leq l \leq n$; and $Z_{l}$ is independent of $\mathcal{F}_{l}, 1 \leq l \leq n$.

Now. consider the estimation of $\rho$. It is well-known that the least squares estimator of $\rho$ is

$$
\dot{\rho}^{L}=\left(\boldsymbol{X}_{n}^{\prime} \mathcal{X}_{n}\right)^{-1} \mathcal{X}_{n}^{\prime} \boldsymbol{X}
$$

where $X=\left(X_{1}, \cdots, X_{n}\right)^{\prime}$ and

$$
n^{1 / 2}\left(\dot{\rho}^{L}-\rho\right) \stackrel{D}{\rightarrow} \mathcal{N}_{p+1}(0, \Sigma)
$$

where $\Sigma$ is the covariance matrix. 
We define a $\lambda$-outoregression quantile (ARQ) for the model (4.1) following hinul and Saleh (1995). An $\lambda$-autoregression quantile $\hat{\boldsymbol{\rho}}_{n}(\lambda) \in \dot{\mathcal{B}}_{n}(\lambda), 0<1<1$, where $\hat{\boldsymbol{B}}_{n}(\lambda)$ is the set of all solutions of the minimization problem:

$$
\sum g_{\lambda}\left(X_{1}-X_{i-1}^{\prime} b\right)=\min
$$

with respect to $\mathrm{b} \in \mathbb{R}^{p+1}$, where $g_{\lambda}(u)=\lambda u I(u>0)-(1-\lambda) u l(u \leq 0)$, $u \in$ $\mathbb{R}$ and $\lambda \in(0,1)$. Note that $\hat{\rho}_{n}(1 / 2)$ is the well-known least alsolute deviation estimator (LAE) of $\rho$. i.e. it is the autoregression median. In this catse also, at in $\mathrm{KB}(1978), \hat{\mathcal{B}}_{n}(\lambda)$ is the convex hull of all solutions having the form

$$
b_{h}=\mathcal{X}_{h}^{-1} X_{h}
$$

where $h$ is a subset of $\{1,2, \ldots, n\}$ of size $p+1$ and $\boldsymbol{X}_{h}$ is suld-denign mat rix wilt rows $\mathbf{X}_{i-1}^{\prime}, i \in \mathbf{h}$.

We may write the above minimization problem to the following linear program ming problem:

$$
\begin{gathered}
\min \left[\lambda 1_{n}^{\prime} \boldsymbol{r}^{+}+(1-\lambda) \mathbf{1}_{n}^{\prime} \boldsymbol{r}^{-}\right], \mathbf{1}_{n}=(1, \ldots, 1)^{\prime} . \\
\text { subject to } \quad \boldsymbol{X}=\boldsymbol{X}_{n} b+\boldsymbol{r}^{+}-\boldsymbol{r}^{-}, \quad\left(\boldsymbol{b}, \boldsymbol{r}^{+}, \boldsymbol{r}^{-}\right) \in \mathbb{E}^{n+1}, \because_{+}^{n} \times \because_{+}^{\prime}
\end{gathered}
$$

To state the results, we may rewrite the condition $\mathrm{Fl}$ from chapter 2 :

F1 $\quad F_{0}$ is continuous and has positive and continuous density function $f_{0}$ in the neighborhood of $Q_{0}(\lambda)$ for each $\lambda \in(0,1)$.

Now we define for each $\lambda \in(0,1)$

$$
\rho(\lambda)=\rho+Q_{0}(\lambda) e_{1}, \quad e_{1}=(1,0, \cdots, 0)^{\prime}
$$

and the randomly weighted process

$$
T_{n}(t ; \lambda)=n^{-1 / 2} \sum_{i=1}^{n} X_{i-1}\left\{I\left(X_{i}-X_{i-1}^{\prime} t \leq 0\right)-\lambda\right\}
$$


Then, we have

$$
T_{n}\left(\rho(\lambda)+n^{-1 / 2} t ; \lambda\right)=n^{-1 / 2} \sum_{i=1}^{n} X_{i-1}\left\{I\left(Z_{i} \leq Q_{0}(\lambda)+n^{-1 / 2} X_{1-1}^{\prime} t\right)-\lambda\right\}
$$

Also, we set $n^{-1 / 2} X_{i-1}^{\prime} t \equiv \xi_{n i}$ as given in the second paragaph of this section.

Now, we state the following results which are the weaker version of the same results given by Koul and Saleh (1995).

Theorem 4.2.1 Inder the conditions a1, a2 and F1 for each $\lambda \in(0,1)$

$$
n^{1 / 2}\left(\hat{\rho}_{n}(\lambda)-\rho(\lambda)\right)=-\left(\Sigma_{n} q_{0}(\lambda)\right)^{-1} T_{n}(\rho(\lambda) ; \lambda)+o_{1}(1)
$$

Lemma 4.2.2 Under the conditions of Thearem 4.2.1 for cach $\lambda \in(0,1)$

$$
\sup _{\|t\| \leq b}\left\|T_{n}\left(\rho(\lambda)+n^{-1 / 2} t ; \lambda\right)-T_{n}(\rho(\lambda): \lambda)-q_{0}(\lambda) \Sigma_{n} t\right\|=o_{l}(1)
$$

The next Lemma will show that the autoregression quantiles are bounded in probability, i.e

Lemma 4.2.3 Vinder the condition of Theorem 4.2.1, for each fixed $\lambda$.

$$
n^{1 / 2}\left(\hat{\rho}_{n}(\lambda)-\rho(\lambda)\right)=O_{p}(1)
$$

The following corollaries give the asymptotic distribution of ARQs.

Corollary 4.2.4 Assume that the conditions of Theortm 4.2.1 hold. Then,

$$
n^{1 / 2}\left(\hat{\rho}_{n}(\lambda)-\rho(\lambda)\right) \stackrel{\mathcal{I}}{\rightarrow} \mathcal{N}_{p+1}\left(0, \frac{\lambda(1-\lambda)}{q_{0}^{2}(\lambda)} \Sigma^{-1}\right)
$$

Corollary 4.2.5 Let $F_{0}$ be continuous with continuous density $f_{0}$ in the neighborhood of $Q_{0}\left(\lambda_{i}\right)$ with $q_{0}\left(\lambda_{i}\right)>0, i=1, \ldots, k$ and let conditions $a 1$ and a 2 hold. Then, for each $\lambda_{1}, \cdots, \lambda_{k}$ such that $0<\lambda_{1}<\lambda_{2}<\cdots<\lambda_{k}<1$

$$
\left(n^{1 / 2}\left(\hat{\rho}\left(\lambda_{1}\right)-\rho\left(\lambda_{1}\right)\right), \cdots, n^{1 / 2}\left(\hat{\rho}\left(\lambda_{k}\right)-\rho\left(\lambda_{k}\right)\right)\right) \stackrel{\mathcal{D}}{\rightarrow} \mathcal{N}_{p+1 \times k}\left(0, \Omega 3 \Sigma^{-1}\right)
$$

whe re $\boldsymbol{\Omega}=\left[\omega_{1 j}\right]$ and $\alpha_{i j}=\frac{\lambda_{1}-\lambda_{1} \lambda_{2}}{g_{0}\left(\lambda_{4}\right) 9_{0}\left(\lambda_{1}\right)} \quad(i<j)$. 


\subsection{L-estimation of the parameters of a autore- gression model based on a few selected au- toregression quantile}

In this section we consider the L-estimation of the autoregression and scale pa rameters of the autoregressive model (4.1) based on the asymptotic mormality of autoregression quantiles.

Consider the linear model

$$
X=X_{n} \rho+\sigma Z
$$

where $Z=\left(Z_{1}, \cdots, Z_{n}\right)^{\prime}$ consists of $i \cdot i \cdot d$. crror variables with a continuous $\cdot d f$ $F_{0}(z)$ and positive $p d f f_{0}(z), z \in \mathbb{E}$. It is well-known that the least squares unethod leads to the estimator of $\rho$ given by

$$
\tilde{\rho}_{n}^{L}=\left(\mathcal{X}_{n}^{\prime} \mathcal{X}_{n}\right)^{-1} \boldsymbol{X}_{n}^{\prime} \boldsymbol{Y}
$$

and an estimator of $\sigma^{2}$ is given by

$$
S^{2}=\frac{\left(\boldsymbol{X}-\boldsymbol{X}_{n} \dot{\boldsymbol{\rho}}_{n}^{L}\right)^{\prime}\left(\boldsymbol{X}-\boldsymbol{X}_{n} \tilde{\boldsymbol{\rho}}_{n}^{L}\right)}{(n-\boldsymbol{p})}
$$

In this section, we introduce the estimation of $\left(\rho^{\prime}, \sigma\right)^{\prime}$ bascod on a few scelectod autoregression quantiles (ARQ). This study will extend the work of (Ogatwa (1951) on the estimation of location and scale model leading to simplifiod estimation of the autoregression and scale parameters of the model (4.15). Our ain is to profose. asymptotically best linear (in ARQ) unbiased estimators (ABLVIEs) of $\rho$ and $\sigma$ based on a few fixed, say $k,(p+2 \leq k<n)$, selected autoregression quantilis (ARQ) under the conditions a1 arid a2.

For a given integer $k(p+2 \leq k<n)$. consider the spacing vector $\lambda \quad\left(\lambda_{1}, \cdots, \lambda_{k}\right)^{\prime}$ whose components satisfy the constraint $0<\lambda_{1}<\cdots<\lambda_{k}=1$. Now, by tho 
minimization problem in the autoregression case similar to (3.4), we obtain the $k$ ARQs given by the vector

$$
\hat{\boldsymbol{\rho}}_{j n}(\lambda)=\left(\hat{\rho}_{j n}\left(\lambda_{1}\right), \ldots, \hat{\rho}_{j_{n}}\left(\lambda_{k}\right)\right)^{\prime}, \quad j=0,1, \ldots, p
$$

Then, using the Corollary 4.2 .5 , one finds that the $k(p+1)$-dimensional random variable

$$
n^{1 / 2}\left(\left(\hat{\rho}_{0 n}(\lambda)-\rho_{0} \mathbf{1}_{k}-\sigma u\right)^{\prime},\left(\dot{\rho}_{1 n}(\lambda)-\rho_{1} \mathbf{1}_{k}\right)^{\prime} \ldots,\left(\dot{\rho}_{p n}(\lambda)-\rho_{p} \mathbf{1}_{k}\right)^{\prime}\right)^{\prime}
$$

converges in law to the $k(p+1)$ dimensional normal distribution with mean 0 and covariance matrix $\sigma^{2}\left(\boldsymbol{\Sigma}^{-1} \otimes \boldsymbol{\Omega}\right)$ where $u=\left(u_{1}, \cdots, u_{k}\right)^{\prime}, u_{j}=Q_{v}\left(\lambda_{j} \quad=1, \ldots k\right.$ and $1=(1, \cdots, 1)^{\prime}$ a $k$-tuple of ones, and $\Omega$ is given in (4.14).

\subsubsection{Joint Estimation of Autoregression and Scale Param- eters}

In this subsection, we propose asymptotically best linear (in $A R Q$ ) unbiased eslimators (ABLUEs) of $\left(\rho^{\prime}, \sigma\right)^{\prime}$. ABLUE of $\left(\rho_{0}, \rho_{1}, \ldots, \rho_{p}\right)^{\prime}$ and $\sigma$ may be obtained by minimizing the quadratic form using (4.19) as

$$
\sum_{i=0}^{p} \sum_{j=0}^{p} S_{i+1, j+1}\left(\dot{\theta}_{2 n}-\theta_{i}\right)^{\prime} \Omega^{-1}\left(\hat{\theta}_{3 n}-\theta_{j}\right),
$$

where $S_{i}$, is the $i j^{\text {th }}$ element of the matrix $\Sigma_{n}$, and by setting

$$
\hat{\boldsymbol{\theta}}_{\mathrm{on}}=\hat{\boldsymbol{\rho}}_{0}(\lambda), \quad \boldsymbol{\theta}_{0}=\rho_{0} \mathbf{1}_{k}+\sigma \boldsymbol{u}, \quad \hat{\boldsymbol{\theta}}_{\mathrm{jn}}=\hat{\boldsymbol{\rho}}_{3 n}(\lambda), \quad \boldsymbol{\theta},=\rho_{j} \mathbf{1}_{k},
$$

for $j=0,1, \cdots, p$. The normal equations are then given by

$$
\left[\begin{array}{cc}
K_{1} \Sigma_{n} & m K_{3} \\
K_{3} m^{\prime} & K_{2}
\end{array}\right]\left[\begin{array}{c}
\hat{\rho}_{n} \\
\tilde{\sigma}_{n}
\end{array}\right]=\left[\begin{array}{c}
\Sigma_{n} V \\
m^{\prime} V^{* *}
\end{array}\right] ; \quad m=\left(1, \bar{X}_{1}, \ldots, \bar{X}_{p}\right)^{\prime}
$$


where

$$
\bar{X}_{j}=n^{-1} \sum_{i=1}^{n} x_{i-n}
$$

The explicit expressions for $\hat{\rho}_{n}$ and $\dot{\sigma}_{n}$ are given by

$$
\begin{aligned}
\dot{\rho}_{n} & =\frac{1}{\Delta}\left[K_{2} V-K_{3} V^{*}\right] \\
\text { and } \quad \dot{\sigma}_{n} & =\frac{1}{\Delta}\left[h_{1} m^{\prime} V^{*}-K_{3} m^{\prime} V\right]
\end{aligned}
$$

respectively, where

$$
\begin{aligned}
& V=\left(V_{0}^{\prime}, V_{1}, \cdots, V_{p}\right)^{\prime}, \quad V_{j}^{\prime}=\mathbf{1}_{k}^{\prime} \boldsymbol{\Omega}^{-1} \dot{\boldsymbol{\rho}}_{j u}(\lambda), \quad(j=0.1, \cdots, p) \\
& V^{*}=\left(V_{0}^{*}, l_{1}^{*}, \cdots, V_{p}^{*}\right)^{\prime}, I_{j}^{*}=u^{\prime} \Omega^{-1} \dot{\rho}_{, \ldots}(\lambda),(j=1,1, \cdots, p)(1,27) \\
& h_{1}=1_{k}^{\prime} \Omega^{-1} 1_{k} . \quad h_{2}=u^{\prime} \Omega^{-1} u . \quad h_{3}=1_{h}^{\prime} \Omega^{-1} u .
\end{aligned}
$$

and $\Delta=\boldsymbol{K}_{1} \boldsymbol{H}_{2}-\boldsymbol{h}_{3}^{2}$.

The asymptotic covariance matrix of $\left(\hat{\boldsymbol{\rho}}_{n}^{\prime}, \hat{\sigma}_{n}\right)^{\prime}$ is given $\mathrm{l}_{\mathrm{y}}$

$$
\frac{\sigma^{2}}{n \Delta}\left[\begin{array}{cc}
K_{2} \Sigma^{-1} & -K_{3} \Sigma^{-1} m \\
-K_{3} m^{\prime} \Sigma^{-1} & K_{1}
\end{array}\right]
$$

Also, the explicit forms of $h_{1}, K_{2}$ and $K_{3}$ are respectively given l,y

$$
\begin{aligned}
& K_{1}=\sum_{i=1}^{k+1} \frac{\left[q_{0}\left(\lambda_{i}\right)-q_{0}\left(\lambda_{t-1}\right)\right]^{2}}{\lambda_{i}-\lambda_{i-1}} \\
& h_{2}=\sum_{i=1}^{k+1} \frac{\left[g_{0}\left(\lambda_{1}\right) Q_{0}\left(\lambda_{i}\right)-g_{0}\left(\lambda_{i-1}\right) Q_{0}\left(\lambda_{1-1}\right)\right]^{2}}{\lambda_{1}-\lambda_{1-1}} \text {, } \\
& K_{3}=\sum_{i=1}^{k+1} \frac{\left[g_{0}\left(\lambda_{i}\right)-g_{0}\left(\lambda_{i-1}\right)\right]\left[g_{0}\left(\lambda_{i}\right) Q_{U}\left(\lambda_{2}\right)-q_{0}\left(\lambda_{i-1}\right) Q_{U}\left(\lambda_{i-1}\right)\right]}{\lambda_{1}-\lambda_{2-1}}
\end{aligned}
$$

with $\lambda_{0}=0$ and $\lambda_{k+1}=1$. If $F_{0}$ is symmetric and $\lambda_{2}=\lambda_{k-t+1}, i=1, \cdots, k$, th:t1 $K_{3}=0$ and the ABLUE of $\rho$ and $\sigma$ simplify to

$$
\tilde{\rho}_{n}=\frac{V}{K_{1}} \quad \text { and } \quad \tilde{\sigma}_{n}=\frac{m^{\prime} V^{\prime}}{K_{2}}
$$


with variance and covariance given by

$$
\operatorname{Var}\left(\tilde{p}_{n}\right)=\frac{\sigma^{2}}{n K_{1}} \Sigma^{-1}, \quad \operatorname{Var}\left(\tilde{\sigma}_{n}\right)=\frac{\sigma^{2}}{n K_{2}^{\prime}}
$$

and

$$
\operatorname{Cov}\left(\hat{\boldsymbol{\rho}}_{n}, \tilde{\sigma}_{n}\right)=0 .
$$

In general the joint asymptotic relative efficiency (JARE) of the ABLLE $\left(\hat{\boldsymbol{\rho}}_{n}^{\prime}, \dot{\sigma}_{n}\right)^{\prime}$ relative to the LSE $\left(\dot{\boldsymbol{\rho}}_{n}^{L \prime}, \dot{\sigma}_{n}^{L}\right)^{\prime}$ may be computed as

$$
J A R E[A B L U E: L S E]=\frac{h_{1}^{p} \Delta}{I_{11}^{p}\left(I_{11} I_{22}-I_{12}^{2}\right)}
$$

and

$$
\begin{aligned}
A R E\left[\hat{\rho}_{n}, L S E\right] & =\frac{I_{22}^{p+1} \Delta^{p+1}}{K_{2}^{p+1}\left(J_{11} I_{22}-I_{12}^{2}\right)^{p+1}} \\
\text { and } \left.A R E\left[\hat{\sigma}_{n}\right), L S E\right] & =\frac{I_{11} \Delta}{K_{1}\left(J_{11} I_{22}-I_{12}^{2}\right)}
\end{aligned}
$$

where $I=\left(\left(I_{2},\right)\right)$ is the information matrix of the location and scale distribution. Another method of estimating $\left(\rho_{0}, \sigma\right)$ and $\rho$ would be to consider two marginal dist ributions, namely, the marginals of

$$
\begin{aligned}
& \text { (i) } n^{1 / 2}\left(\hat{\rho}_{0 n}(\lambda)-\rho_{0} 1_{k}-\sigma u\right)^{\prime} \\
& \text { (ii) } n^{1 / 2}\left(\left(\hat{\rho}_{1 n}(\lambda)-\rho_{1} 1_{k}\right)^{\prime}, \cdots,\left(\hat{\rho}_{p n}(\lambda)-\rho_{p} 1_{k}\right)^{\prime}\right)^{\prime}
\end{aligned}
$$

The first one yields the estimators

$$
\begin{aligned}
& \tilde{\tilde{\rho}}_{0 n}=\frac{1}{\Delta}\left\{K_{2} V_{0}-K_{3} V_{0}^{r_{0}^{*}}\right\} . \\
& \dot{\tilde{\sigma}}_{n}=\frac{1}{\Delta}\left\{K_{1} V_{0}^{*}-K_{3} V_{0}^{\prime}\right\}
\end{aligned}
$$

and the second one yields the estimators of $\rho$ as

$$
\dot{\hat{\rho}}_{n}=\frac{1}{h_{1}} V, \quad V=\left(V_{1}^{\prime}, \cdots, V_{p}\right)^{\prime}
$$


The estimator $\grave{\hat{\sigma}}_{n}$ of $\sigma$ is non-neguive but (4.25) may not 'we non- negative. The ARl: of these estimators relative to the corresponding marginal likelihood est imators may be determined easily.

Now consider the explicit expressions for the coefficien's in $(4.24)$ and $(1.10)$. They are

$$
\begin{aligned}
& a=\frac{1}{\Delta}\left\{K_{2} \mathbf{1}_{k}^{\prime} \boldsymbol{\Omega}^{-1}-\boldsymbol{K}_{3} \boldsymbol{u}^{\prime} \boldsymbol{\Omega}^{-1}\right\}=\left(a_{1}, \cdots, a_{k}\right)^{\prime} \\
& \boldsymbol{b}=\frac{1}{\Delta}\left\{\boldsymbol{K}_{1} \boldsymbol{u}^{\prime} \boldsymbol{\Omega}^{-1}-\boldsymbol{K}_{3} \boldsymbol{1}_{k}^{\prime} \boldsymbol{\Omega}^{-1}\right\}=\left(b_{1}, \cdots, b_{k}\right)^{\prime}
\end{aligned}
$$

where for $i=1,2, \ldots, k$ we have

$$
\begin{aligned}
& a_{2}=\frac{q_{0}\left(\lambda_{2}\right) K_{2}}{\Delta}\left\{\frac{q_{0}\left(\lambda_{i}\right)-q_{0}\left(\lambda_{i-1}\right)}{\lambda_{i}-\lambda_{1-1}}-\frac{q_{0}\left(\lambda_{t+1}\right)-q_{0}\left(\lambda_{1}\right)}{\lambda_{1+1}-\lambda_{1}}\right\} \\
& \frac{q_{0}\left(\lambda_{i}\right) K_{3}}{\Delta}\left\{\frac{q_{0}\left(\lambda_{2}\right) Q_{0}\left(\lambda_{1}\right)-q_{0}\left(\lambda_{1-1}\right) Q_{0}\left(\lambda_{t-1}\right)}{\lambda_{1}-\lambda_{1-1}}-\frac{q_{0}\left(\lambda_{i+1}\right) Q_{0}\left(\lambda_{1+1}\right)-q_{01}\left(\lambda_{1}\right) Q_{01}\left(\lambda_{1}\right)}{\lambda_{i+1}-\lambda_{1}}\right\} \\
& b_{s}=\frac{q_{0}\left(\lambda_{i}\right) K_{1}}{\Delta}\left\{\frac{q_{0}\left(\lambda_{i}\right) Q_{0}\left(\lambda_{i}\right)-q_{0}\left(\lambda_{t-1}\right) Q_{0}\left(\lambda_{t-1}\right)}{\lambda_{i}-\lambda_{t-1}}-\frac{q_{0}\left(\lambda_{t+1}\right) Q_{0}\left(\lambda_{t+1}\right)-q_{01}\left(\lambda_{1}\right) Q_{0}\left(\lambda_{0}\right)}{\lambda_{t+1}-\lambda_{1}}\right\} \\
& -\frac{q_{0}\left(\lambda_{1}\right) h_{3}}{\Delta}\left\{\frac{q_{0}\left(\lambda_{1}\right)-q_{0}\left(\lambda_{t-1}\right)}{\lambda_{1}-\lambda_{t-1}}-\frac{q_{0}\left(\lambda_{t+1}\right)-q_{0}\left(\lambda_{1}\right)}{\lambda_{1+1}-\lambda_{1}}\right\} \text {. }
\end{aligned}
$$

Using these coefficients, we may write the estimators (-1.24) and (4.25) as follows:

$$
\left[\begin{array}{c}
\tilde{\rho}_{0 n} \\
\tilde{\rho}_{1 n} \\
\vdots \\
\hat{\rho}_{p n} \\
\tilde{\sigma}_{n}
\end{array}\right]=\left[\begin{array}{c}
a_{1} \hat{\rho}_{u n}\left(\lambda_{1}\right)+\cdots+a_{k} \dot{\rho}_{1 m}\left(\lambda_{k}\right) \\
a_{1} \hat{\rho}_{1 n}\left(\lambda_{1}\right)+\cdots+a_{k} \dot{\rho}_{1 n}\left(\lambda_{k}\right) \\
\vdots \\
a_{1} \dot{\rho}_{p n}\left(\lambda_{1}\right)+\cdots+a_{k} \hat{\rho}_{2 n}\left(\lambda_{k}\right) \\
\sum_{J=0}^{p} \bar{X}_{j}\left(b_{1} \dot{\rho}_{j n}\left(\lambda_{1}\right)+\cdots+b_{k} \dot{\rho}_{j n}\left(\lambda_{k}\right)\right.
\end{array}\right] .
$$

Once we know the optimum spacing vector $\lambda^{0}=\left(\lambda_{1}^{0}, \ldots, \lambda_{k}^{0}\right)$, we can determine. $\hat{\rho}_{j n}\left(\lambda_{i}^{0}\right), j=0,1, \ldots, p$ for each $i=1, \ldots, k$, and the coefficients $\left(a_{1}, \ldots, a_{k}\right)^{\prime}$ and $\left(b_{1}, \ldots, b_{k}\right)^{\prime}$.

Tables in appendix A provide some optimum spacings when the errors are distributad as $t$-distribution and logistic distribution. 
Now we present a numerical example using the computational algorithms of Kownker and d'Orey (198i, 1994) extended to the autoregression case for computing ARQs'.

Example 4.3.1 Consider the following 100 observations from autorgressive model of orde; one (AR(I)) given in example 7.3 by Fuller (1976). He gencrated these data with independent normal $(0.1)$ errors.

$\begin{array}{cccccccccc}0.874 & 0.850 & 2.345 & 2.501 & 1.657 & 1.649 & 2.498 & 1.330 & 1.307 & 3.10 .1 \\ 2.445 & 2.805 & 1.639 & 1.240 & 1.116 & 0.448 & 0.377 & -0.488 & -0.96 & -0.579 \\ -1.674 & -0.366 & -0.922 & -1.174 & -1.585 & -0.613 & 0.110 & 0.113 & -0.305 & 0.723 \\ -0.257 & 1.051 & 0.803 & 0.116 & -1.454 & 0.296 & 1.501 & 0.880 & -0.672 & 0.436 \\ 0.930 & 1.168 & 1.999 & 1.376 & 1.613 & 2.030 & 0.616 & 0.667 & 0.707 & 1.029 \\ -0.366 & -1.420 & -0.183 & -0.044 & -0.391 & -0.095 & -0.971 & 0.371 & -1.622 & -2.941 \\ -2.814 & -1.784 & -2.471 & -3.508 & -2.979 & -0.779 & 0.869 & 1.786 & 0.123 & 0.093 \\ -0.731 & -1.253 & -2.213 & -0.252 & 0.403 & -0.995 & -0.948 & 0.046 & 0.091 & 0.254 \\ 2.750 & 1.673 & 2.286 & 1.220 & -0.256 & 0.252 & 0.325 & -0.338 & 0.378 & 0.127 \\ -2.006 & -2.380 & -2.024 & -1.085 & 1.037 & -0.467 & -0.794 & -0.493 & -0.157 & 0.659\end{array}$

In this rase the model is

$$
X_{t}=\rho_{0}+\rho_{1} X_{t-1}+Z_{t}
$$

The LSE of the parameters $\left(\rho_{0}, \rho_{1}\right)$ is $(0.0259,0.7434)$ and the predicted ralues based 
CHAPTER 4. ALTOREGRESSION MODEI.

on this estimator are

$\begin{array}{llllllllll}0.026 & 0.676 & 0.658 & 1.769 & 1.885 & 1.258 & 1.252 & 1.853 & 1.015 & 0.9495\end{array}$ $\begin{array}{llllllllll}2.556 & 1.844 & 2.111 & 1.244 & 0.948 & 0.856 & 0.359 & 0.306 & -0.337 & \cdots\end{array}$

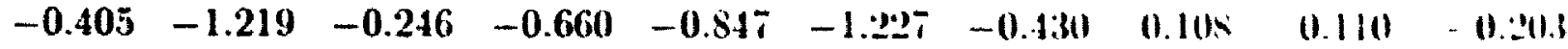

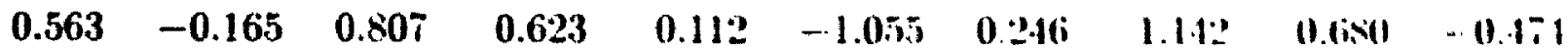
$\begin{array}{llllllllll}0.350 & 0.717 & 0.894 & 1.512 & 1.049 & 1.225 & 1.535 & 0.414 & 0.52 .2 & 0.501\end{array}$

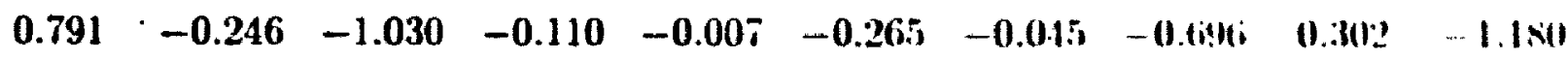

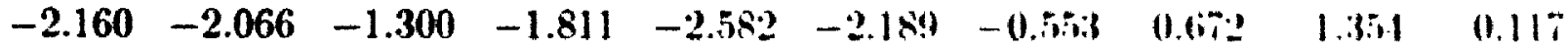

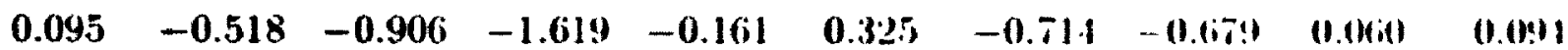

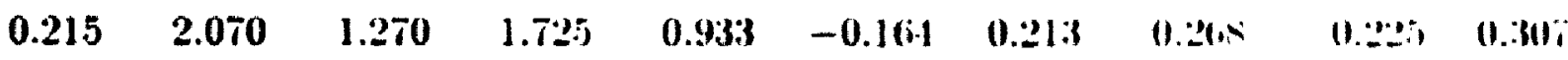
$\begin{array}{llllllllll}0.120 & -1.465 & -1.743 & -1.479 & -0.781 & 0.797 & -0.321 & -0.561 & 0.311 & 0.031\end{array}$

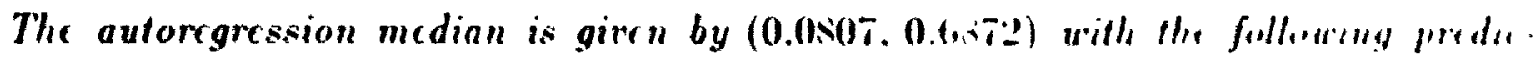
tions:

\begin{tabular}{|c|c|c|c|c|c|c|c|c|c|}
\hline 0.081 & 0.681 & 0.665 & 1.692 & .899 & 219 & 1.21 .1 & דית & $99 \%$ & 1.979 \\
\hline 2.420 & 61 & 008 & - & 8 & 0.8 .48 & 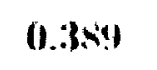 & 11 & $\therefore$ & :u \\
\hline .317 & .070 & 0.171 & 3 & $6 i$ & 7 & 1 & i & $0.15 \mathrm{k}$ & $0.1: 31$ \\
\hline 0.578 & -0.096 & 0.803 & 0.633 & 0.160 & ה & $0.2 \times 1$ & 1.112 & (0.6is:) & $0.3 x$ \\
\hline 0.380 & 0.720 & 0.883 & 154 & aor & $1 \times 0$ & 1.176 & 1 & $0.5 \times 4$ & 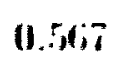 \\
\hline 0.788 & 1 & .8 & -0.0 .45 & $0.0 \div 0$ & 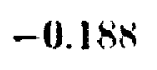 & 0.0 & $i$ & 0.3346 & 1.10 .11 \\
\hline-1.940 & -1.853 & -1.145 & $-1.61 i$ & -2.330 & $-1.9666_{0}$ & -0.455 & Q.tiTs & 1.:3rk & (1.160\% \\
\hline 0.145 & -0.422 & -0.780 & -1.440 & -0.092 & 0.358 & $-0.60: 3$ & $-0.5 \pi 1$ & 0.112 & 0.1 .13 \\
\hline .255 & 1.971 & 1.230 & 1.652 & 0.919 & 0.095 & 0.25 & 0.3011 & -0.172 & . \\
\hline 0.168 & -1.298 & -1.555 & -1.310 & -0.665 & 0.793 & -0.240 & 0.465 & $-0.2 \sin$ & (1.0.192 \\
\hline
\end{tabular}

Plot 4.1 shows these two results comparing with the original datu as frollow: 


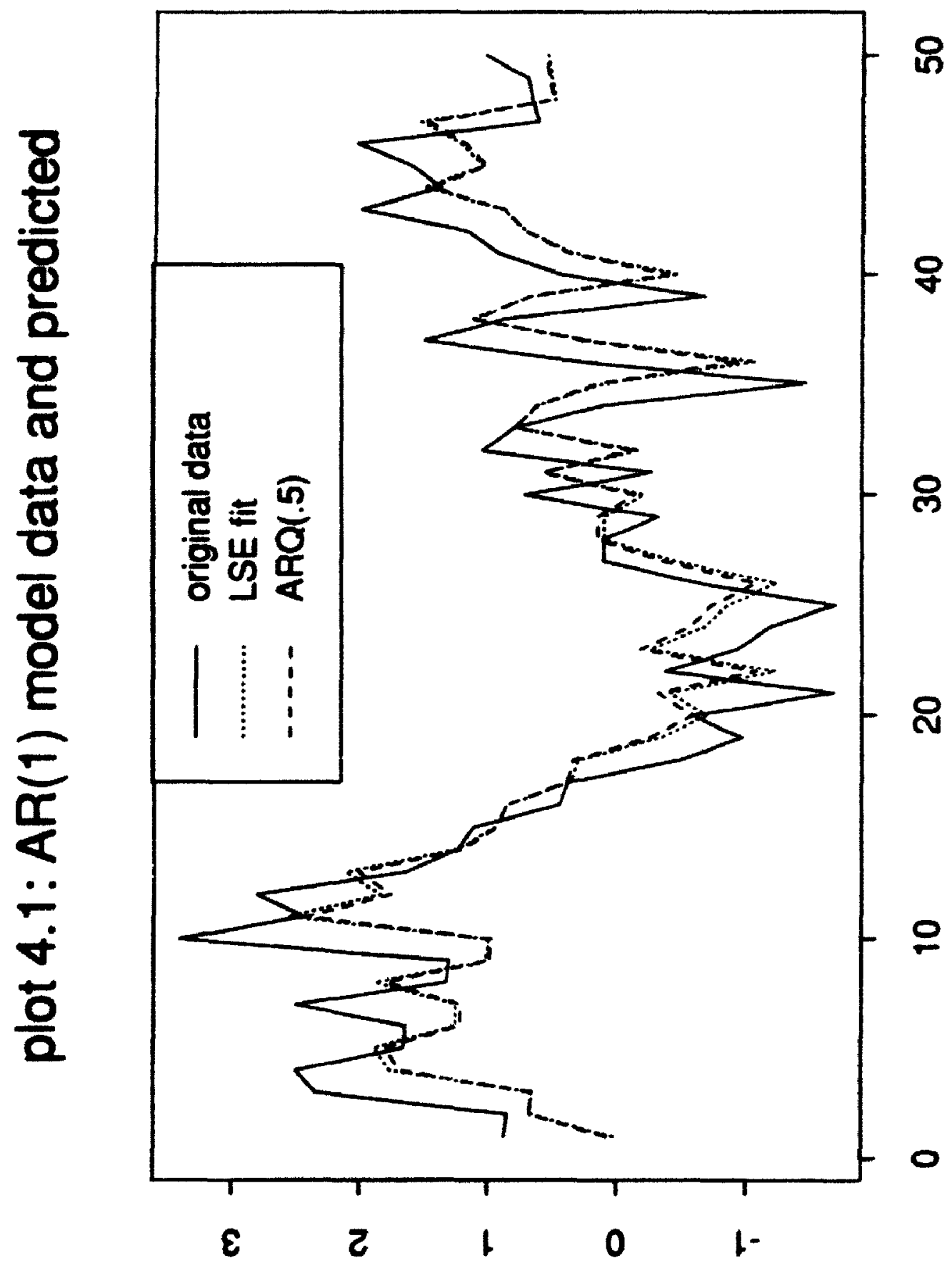




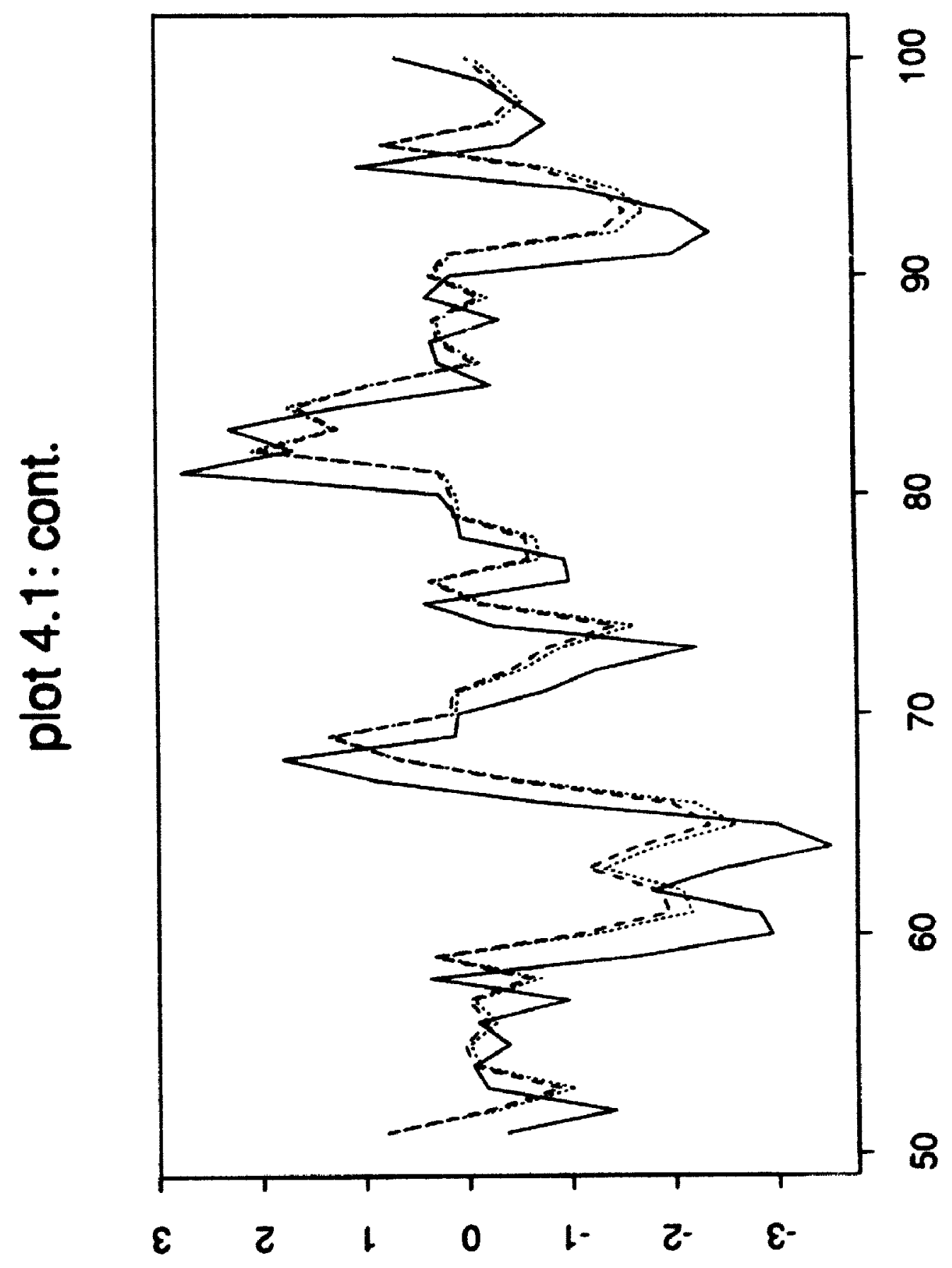




\section{Example 4.3.2 Gastuiths' Estimator:}

The Gast wirth estimator is the linear combination of $1 / 3,1 / 2$ and $2 / 3$ autoregres. sion quantiles with the coefficient $.3, .4$ and .3 respectively. The estimator of parameters based on this method is $(0.0396,0.696)$ with the following prediction values

$\begin{array}{cccccccccc}0.040 & 0.648 & 0.631 & 1.672 & 1.780 & 1.193 & 1.187 & 1.778 & 0.965 & 0.949 \\ 2.409 & 1.741 & 1.992 & 1.180 & 0.903 & 0.816 & 0.351 & 0.302 & -0.300 & -0.629 \\ -0.363 & -1.126 & -0.215 & -0.602 & -0.778 & -1.133 & -0.387 & 0.116 & 0.118 & -0.175 \\ 0.543 & -0.139 & 0.771 & 0.598 & 0.120 & -0.972 & 0.246 & 1.08 . & 0.652 & -0.428 \\ 0.343 & 0.687 & 0.853 & 1.431 & 0.997 & 1.162 & 1.452 & 0.468 & 0.50 .1 & 0.532 \\ 0.756 & -0.215 & -0.949 & -0.088 & 0.009 & -0.233 & -0.027 & -0.636 & 0.298 & -1.089 \\ -2.007 & -1.919 & -1.202 & -1.680 & -2.402 & -2.034 & -0.503 & 0.64 . & 1.283 & 0.125 \\ 0.104 & -0.469 & -0.832 & -1.501 & -0.136 & 0.320 & -0.653 & -0.620 & 0.072 & 0.103 \\ 0.216 & 1.954 & 1.204 & 1.631 & 0.889 & -0.139 & 0.215 & 0.266 & -0.196 & 0.303 \\ 0.128 & -1.357 & -1.617 & -1.369 & -0.716 & 0.761 & -0.285 & -0.513 & -0.304 & -0.070\end{array}$

(iraphical display of these results together with autorgression median and original data is giren in plot 4.2 . 


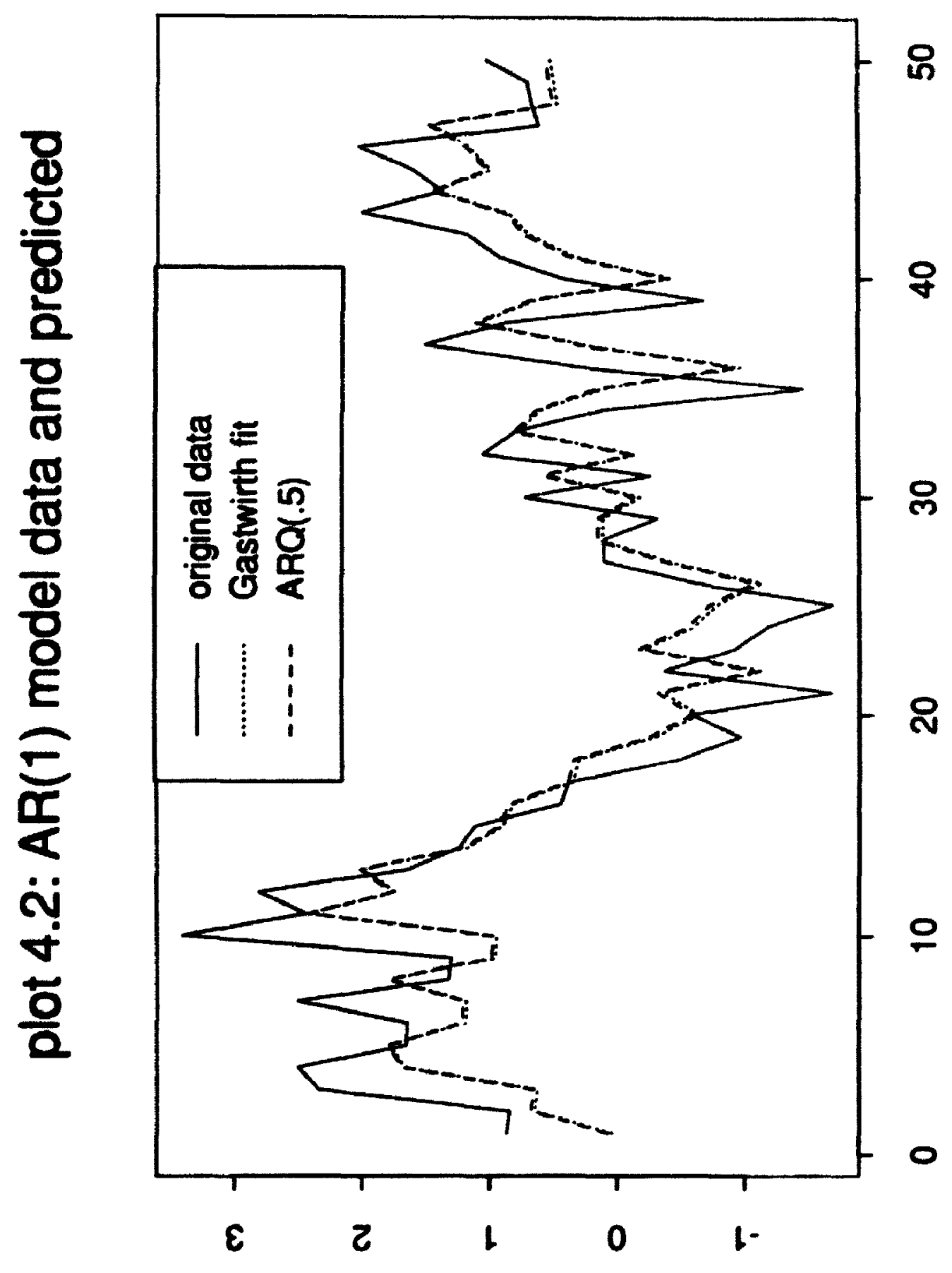




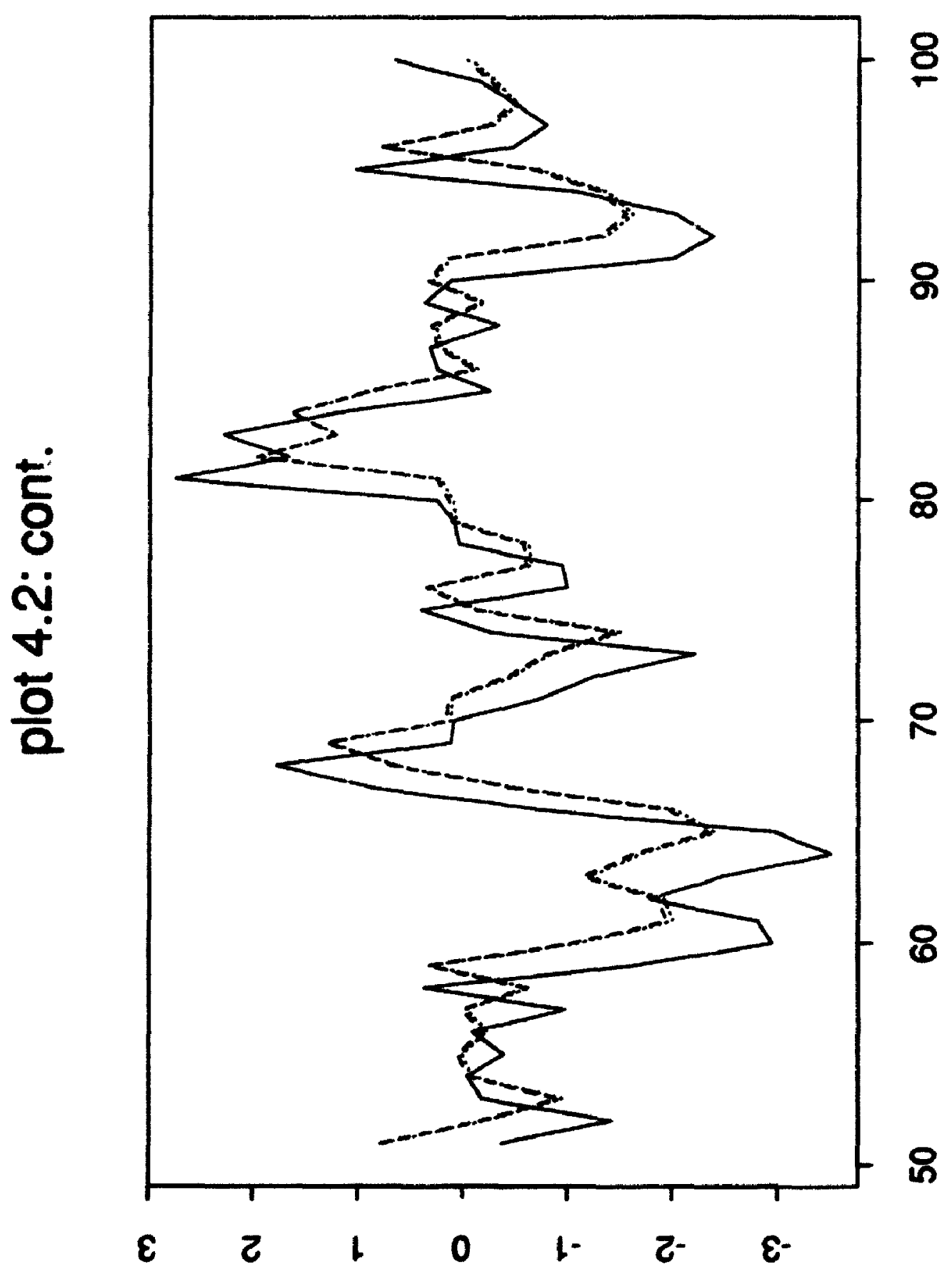


Example 4.3.3 Trimmed LSE and Estimation based on 7 RQs.

Estimate the parameters based on a few selected autorgression quantiles and trimmod least squares estimator are two L-estimators which ue shall estimate $\left(\mu_{0}, p_{1}\right)$ based on these two methods. The $10 \%$ TLSE of the parameters is $(.0138, .7273)$ and the corresponding predicted values are

\begin{tabular}{|c|c|c|c|c|c|c|c|c|c|}
\hline .014 & 0.649 & .632 & .719 & 1.833 & 1.219 & 1.213 & $3: 31$ & .981 & 0.96 .1 \\
\hline 0 & 1.792 & $\boldsymbol{t}$ & 6 & 0 & 5 & 0 & S & 11 & 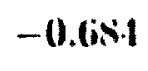 \\
\hline 0.407 & -1.204 & -0.252 & $-0.65 i$ & -0.840 & -1.212 & -0.432 & 0.091 & 0.0946 & -0.210 \\
\hline 0.540 & -0.173 & 0.778 & 0.598 & 0.098 & -1.044 & 0.229 & 1.105 & 0.654 & -0.175 \\
\hline .331 & 0.690 & 0.863 & $46 \mathrm{~s}$ & .015 & $1.18 i$ & 1.490 & $0.46:$ & $0.49 !$ & 0.58 \\
\hline 0.762 & -0.252 & -1.019 & -0.119 & -0.018 & -0.271 & -0.055 & -0.69 .2 & $0.2+4$ & -1.166 \\
\hline 2.125 & -2.033 & -1.284 & 783 & -2.538 & - & -0.553 & 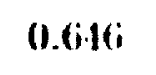 & 1.31:3 & 1118 \\
\hline 0.081 & -0.518 & -0.898 & -1.596 & -0.169 & $0.30 \mathrm{~T}$ & -0.710 & -0.676 & 0.0 .17 & 0.0180 \\
\hline 0.199 & 2.014 & 1.231 & 1.676 & 0.901 & -0.172 & 0.197 & 0.250 & -0.232 & 0.289 \\
\hline 0.106 & -1.445 & -1.717 & -1.458 & -0.775 & 0.768 & -0.326 & -0.56 .1 & $-0.3 \cdot 15$ & -0.100 \\
\hline
\end{tabular}

The estimation of parameters based on 7 optimal autorgression guantile is (0.020.1, .731) with the following prediction valucs

$\begin{array}{cccccccccc}0.020 & 0.659 & 0.642 & 1.735 & 1.849 & 1.232 & 1.226 & 1.816 & 0.9983 & 0.976 \\ 2.509 & 1.808 & 2.071 & 1.219 & 0.927 & 0.836 & 0.348 & 0.296 & -0.3336 & -0.6 \times 1 \\ -0.403 & -1.203 & -0.247 & -0.654 & -0.838 & -1.211 & -0.428 & 0.101 & 0.103 & -0.205 \\ 0.549 & -0.167 & 0.789 & 0.607 & 0.105 & -1.042 & 0.237 & 1.118 & 0.664 & -0.471 \\ 0.339 & 0.700 & 0.874 & 1.482 & 1.026 & 1.200 & 1.504 & 0.471 & 0.508 & 0.537 \\ 0.773 & -0.247 & -1.018 & -0.113 & -0.012 & -0.26 .5 & -0.049 & -0.6 \times 9 & 0.292 & -1.165 \\ -2.129 & -2.037 & -1.284 & -1.786 & -2.544 & -2.157 & -0.549 & 0.656 & 1.326 i & 0.110 \\ 0.088 & -0.514 & -0.896 & -1.597 & -0.164 & 0.315 & -0.707 & -0.673 & 0.054 & 0.057 \\ 0.206 & 2.031 & 1.243 & 1.691 & 0.912 & -0.167 & 0.205 & 0.258 & -0.227 & 0.297 \\ 0.113 & -1.446 & -1.719 & -1.459 & -0.773 & 0.778 & -0.321 & -0.560 & -0.340 & -0.694\end{array}$

Plot 4.3 shows these two results as well as the original data. 


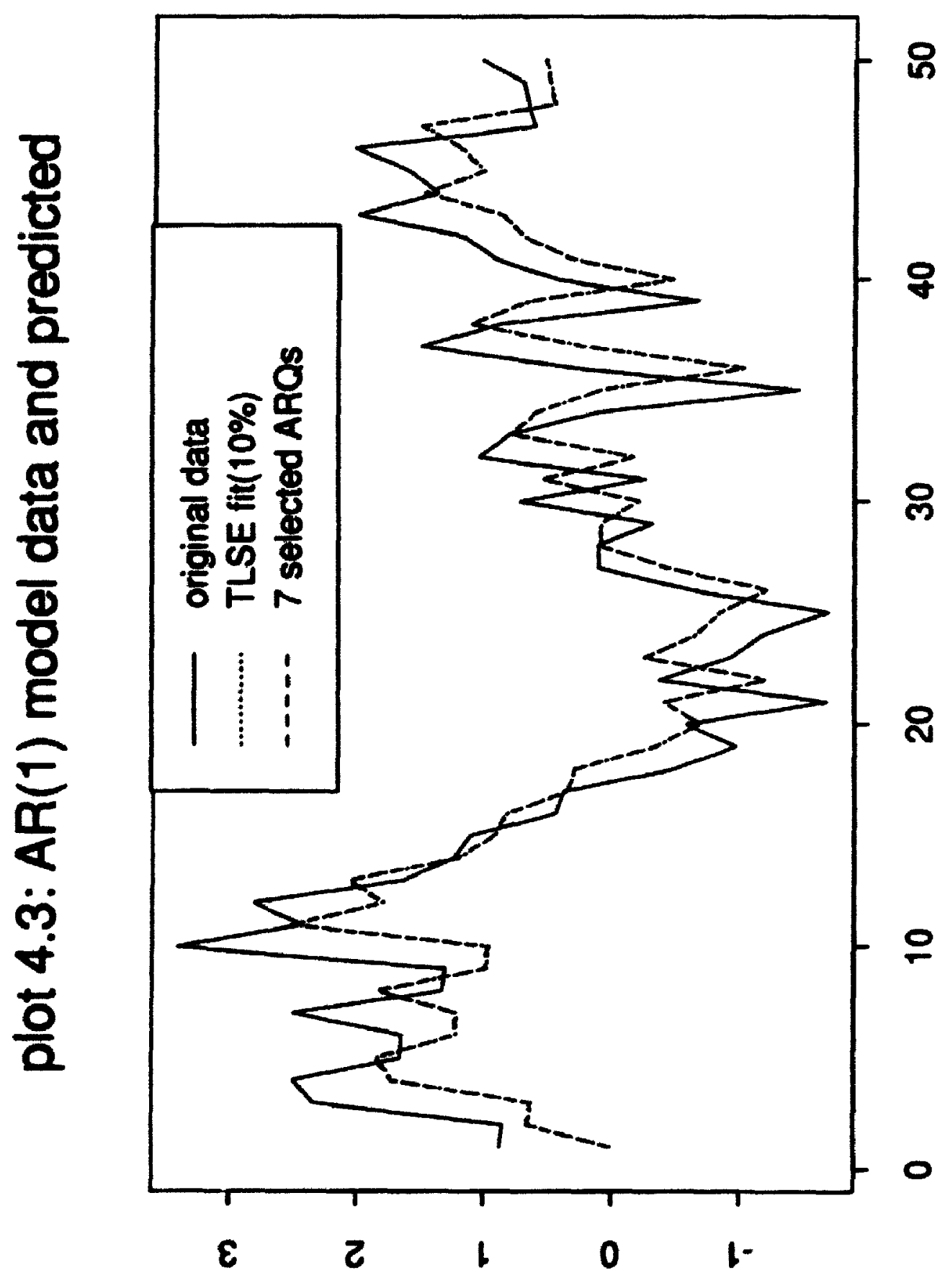




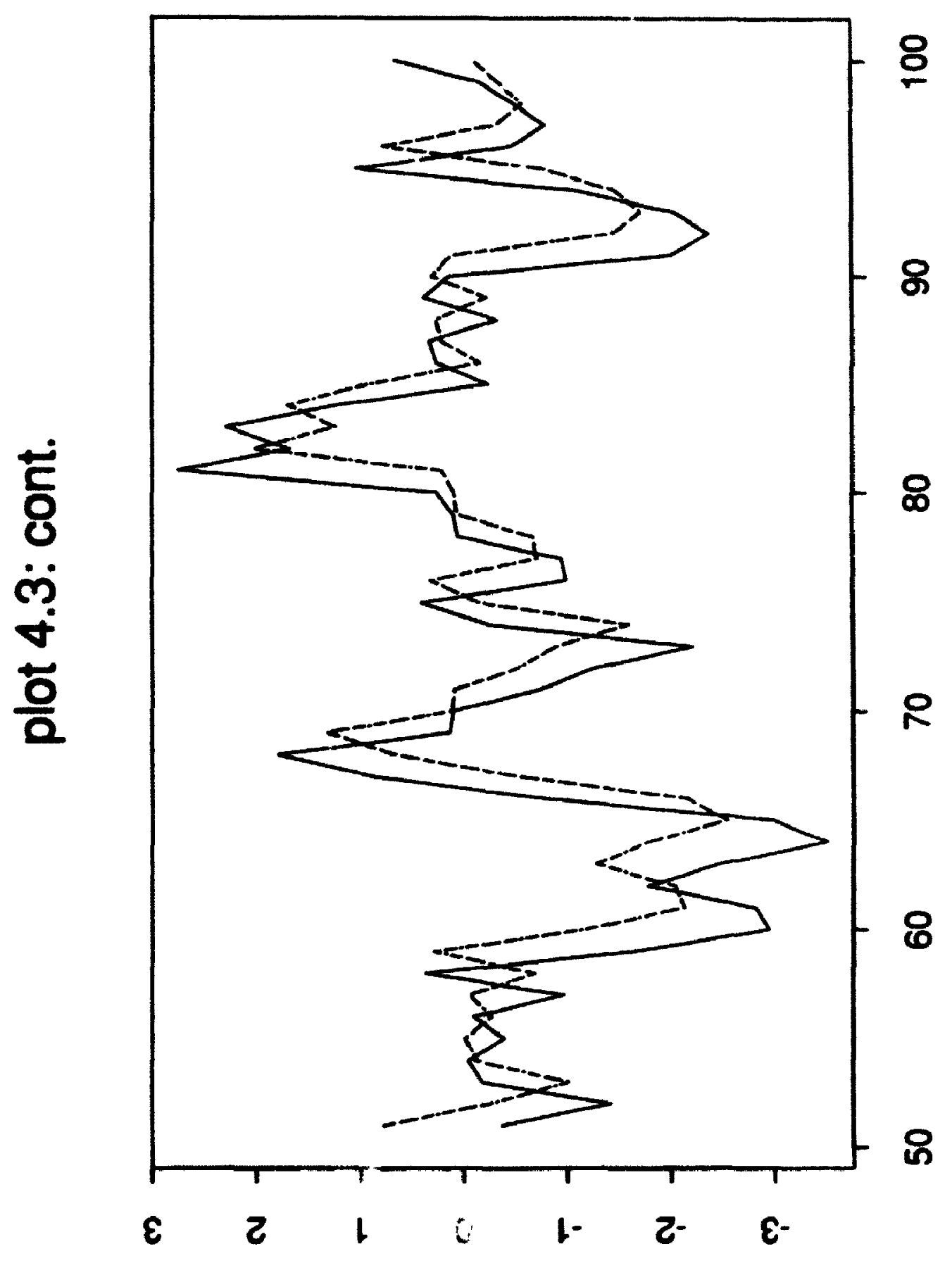




\subsection{Trimmed Least Squares Estimation of $\mathbf{A R}(p)$ Parameters}

In Section 4.3, we studied the L-estimators of the autoregression parameters under known error distribution based on a few selected autoregression quantiles. In this section, we shall consider another L-estimator of $p$ when $F_{0}$ is unknown. It is the Trimmed Least Squares Estimator (TLSE) of the autoregression parameters. To obtain the TLSE of $\rho$, we consider the autoregression model (4.2) and define an $n \times n$ diagonal matrix $A$ whose elements are

$$
a_{11}=\left\{\begin{array}{cc}
0, & \text { if } X_{i} \leq X_{i-1}^{\prime} \hat{\boldsymbol{p}}_{n}\left(\lambda_{1}\right) \text { or } X_{1}>X_{i-1}^{\prime} \hat{\boldsymbol{\rho}}_{n}\left(\lambda_{2}\right) \\
1, & \text { otherwise }
\end{array}\right.
$$

where $\dot{\rho}_{n}\left(\lambda_{1}\right)$ and $\dot{\rho}_{n}\left(\lambda_{2}\right)$ are the autoregression quantiles of orders $\lambda_{1}$ and $\lambda_{2}$ respectively for $0<\lambda_{1}<1 / 2<\lambda_{2}<1$. We obtain the TLSE of $\rho$ by minimizing

$$
\left(X-X_{n} \rho\right)^{\prime} A\left(X-X_{n} \rho\right)
$$

with respect to $\rho$ which yields

$$
\bar{\rho}_{n}\left(\lambda_{1}, \lambda_{2}\right)=\left(\mathcal{X}_{n}^{\prime} A \mathcal{X}_{n}\right)^{-1} \mathcal{X}_{n}^{\prime} A X
$$

We are going to study the asymptotic property of the above estimator under the same condition as in Section 4.2 and via asymptotic linearity. The following main theorem of this section represents the TLSE of $\rho$.

Theorem 4.4.1 Let $F_{0}$ be continuous with positive and continuous density $f_{0}$ in the neighborhood of $Q_{0}\left(\lambda_{1}\right)$ and $Q_{0}\left(\lambda_{2}\right)$. Then, if conditions a1 and a2 hold, for each $\lambda_{1}$ and $\lambda_{2}$ such that $0<\lambda_{1}<1 / 2<\lambda_{2}<1$

$$
\text { i) } n^{1 / 2}\left(\bar{\rho}_{n}\left(\lambda_{1}, \lambda_{2}\right)-\rho\right)=
$$




$$
\begin{array}{r}
n^{-1 / 2}\left\{\left(\lambda_{2}-\lambda_{1}\right) \Sigma_{n}\right\}^{-1} \sum_{i=1}^{n} \mathbf{X}_{1-1}\left(\phi\left(Z_{1}\right)-\vartheta\right)+o_{r}(1) \\
\text { ii) } \quad n^{1 / 2}\left(\bar{\rho}_{n}\left(\lambda_{1}, \lambda_{2}\right)-\rho\right) \stackrel{D}{\rightarrow} \mathcal{N}_{r+1}\left(0, \sigma^{2}\left(\lambda_{1}, \lambda_{2}\right) \Sigma^{-1}\right)
\end{array}
$$

where $\phi\left(Z_{i}\right), \gamma$ and $\sigma^{2}\left(\lambda_{1}, \lambda_{2}\right)$ are defined in (2.97) and (2.98) respectirely.

\section{Proof}

This Theorem will be proved with the aid of two lemmas. First, we define the following processes

$$
\dot{T}_{n}(\mathbf{t} ; \lambda)=n^{-1 / 2} \sum_{i=1}^{n} \mathbf{X}_{t-1} Z_{i} I\left(Z_{i} \leq Q_{U}(\lambda)+n^{-1 / 2} \mathbf{X}_{i-1}^{\prime} t\right)
$$

and

$$
\left.\dot{m}_{n} i i ; \lambda\right)=n^{-1 / 2} \sum_{i=1}^{n} \mathbf{X}_{t-1} \mu\left(Q_{0}(\lambda)+n^{-1 / 2} \mathbf{X}_{t-1}^{\prime} \mathbf{t}\right)
$$

where

$$
\mu(x)=E\left(Z_{1} J\left(Z_{1} \leq x\right) \mid \mathcal{F}_{1}\right)
$$

Then, we define the following center $: d$ process

$$
\begin{aligned}
\dot{\boldsymbol{T}}_{n}^{0}(\mathbf{t} ; \lambda)= & \dot{\boldsymbol{T}}_{n}(\mathbf{t} ; \lambda)-\dot{\boldsymbol{m}}_{n}(\mathbf{t} ; \lambda) \\
=n^{-1 / 2} \sum_{i=1}^{n} \mathbf{X}_{i-1} & \left\{Z_{i} I\left(Z_{i} \leq Q_{0}(\lambda)+n^{-1 / 2} \mathbf{X}_{i-1}^{\prime} t\right)\right. \\
& \left.-\mu\left(Q_{0}(\lambda)+n^{-1 / 2} \mathbf{X}_{i-1}^{\prime} \mathbf{t}\right)\right\}
\end{aligned}
$$

Lemma 4.4.2 Under the condition of Theorem 4.4.1 for each $0<b<x$

$$
\sup _{\|t\| \leq b}\left\|\tilde{T}_{n}(t ; \lambda)-\tilde{T}_{n}(0 ; \lambda)-g_{0}(\lambda) Q_{0}(\lambda) \Sigma_{n} t\right\|=o_{\nu}(1)
$$

\section{Proof}

Consider $\tilde{T}_{n j}(t ; \lambda)$ as the $j^{\text {th }}$ component of $\tilde{T}_{n}(t ; \lambda)$. Then, we have

$$
\sup _{\| t \mid \leq b}\left|\tilde{T}_{n j}(\mathbf{t} ; \lambda)-\tilde{T}_{n j}(\mathbf{0} ; \lambda)-q_{0}(\lambda) Q_{0}(\lambda) \Sigma_{n} \mathbf{t}\right|
$$




$$
\begin{aligned}
\leq & \sup _{\|t\| \leq b}\left|\tilde{T}_{n j}^{0}(t ; \lambda)-\tilde{T}_{n j}^{0}(0 ; \lambda)\right| \\
& +\sup _{\|t\| \leq b}\left|\tilde{m}_{n}(t ; \lambda)-\dot{m}_{n}(0 ; \lambda)-g_{0}(\lambda) Q_{0}(\lambda) \Sigma_{n} t\right| \\
= & J_{1}+J_{2} .
\end{aligned}
$$

First, note that

$$
J_{2} \leq n^{-1 / 2} \sum_{i=1}^{n}\left|X_{i-j}\right| \sup _{\|t\| \leq b}\left|\mu\left(Q_{0}(\lambda)+n^{-1 / 2} X_{i-1}^{\prime} t\right)-\mu\left(Q_{0}(\lambda)\right)-q_{0}(\lambda) Q_{0}(\lambda) n^{-1 / 2} X_{i-1}^{\prime} t\right|
$$

Then, by mean value Theorem there is a $\Delta(t) \in\left(0, n^{-1 / 2} X_{i-1}^{\prime} t\right)$ such that if $t \in$ $\mathcal{N}(b),|\Delta(t)| \leq n^{-1 / 2}\left\|X_{1-1}\right\| b=\delta_{n}$. Therefore,

$$
\begin{aligned}
J_{2} \leq b n^{-1} \sum_{i=1}^{n}\left|X_{i-j}\right|\left\|\mathbf{X}_{i-1}\right\| \sup _{\|t\| \leq b} \mid\left(Q_{0}(\lambda)+\Delta(t)\right) f_{0}\left(Q_{0}(\lambda)+\Delta(t)\right) \\
\quad-Q_{0}(\lambda) f_{0}\left(Q_{0}(\lambda)\right) \mid \\
\leq b n^{-1} \sum_{i=1}^{n}\left|X_{i-j}\right|\left\|\mathbf{X}_{i-1}\right\|\left\{\sup _{|\Delta(t)| \leq \delta_{n}}\left|Q_{0}(\lambda)\left(f_{0}\left(Q_{0}(\lambda)+\Delta(t)\right)-f_{0}\left(Q_{0}(\lambda)\right)\right)\right|\right. \\
\left.\quad+\sup _{|\Delta(t)| \leq \delta_{n}}\left|\Delta(t) f_{0}\left(Q_{0}(\lambda)+\Delta(t)\right)\right|\right\} \\
=b O_{P}(1)\left\{h \cdot o(1)+o_{P}(1) O(1)\right\} \\
=O_{P}(1) \quad \text { as } n \rightarrow \infty
\end{aligned}
$$

by $F 1$ and $C-S$ inequality. To prove Lemma 4.4.2 it is enough to show that $J_{1}=o_{P}(1)$ where

$$
J_{1}=\sup _{\|t\| \leq b}\left|\tilde{T}_{n j}^{0}(\mathbf{t} ; \lambda)-\dot{T}_{n j}^{0}(0 ; \lambda)\right|
$$

First, let $t$ be fixed.

$$
\begin{aligned}
& \left|\dot{I}_{n j}^{0}(\mathbf{t} ; \lambda)-\dot{T}_{n j}^{0}(0 ; \lambda)\right| \\
& =\mid n^{-1 / 2} \sum_{i=1}^{n} X_{i-j}\left\{Z_{i} I\left(Q_{0}(\lambda)<Z_{i} \leq Q_{0}(\lambda)+n^{-1 / 2} \mathbf{X}^{\prime} \quad t\right)\right. \\
& \left.\quad-\mu\left(Q_{0}(\lambda)+n^{-1 / 2} \mathbf{X}_{i-1}^{\prime} t\right)+\mu\left(Q_{0}(\lambda)\right)\right\} \mid
\end{aligned}
$$




$$
=\left|n^{-1 / 2} \sum_{i=1}^{n} x_{1-}\left\{\tilde{l}_{i}(t)-\dot{\nu}_{n i}(t)\right\}\right|
$$

where

$$
\begin{aligned}
& \dot{V}_{i}(t)=Z_{1} I\left(Q_{0}(\lambda)<Z_{i} \leq Q_{0}(\lambda)+n^{-1 / 2} X_{i-1}^{\prime} t\right) \\
& \dot{\nu}_{n t}(t)=\mu\left(Q_{0}(\lambda)+n^{-1 / 2} X_{i-1}^{\prime} t\right)-\mu\left(Q_{0}(\lambda)\right)
\end{aligned}
$$

Therefore,

$$
\begin{aligned}
& P\left\{\left|n^{-1 / 2} \sum_{i=1}^{n} X_{1-},\left\{\dot{V}_{i}(t)-\tilde{\nu}_{n i}(t)\right\}\right|^{2}>c^{2}\right\} \\
& \leq \frac{1}{\epsilon^{2}} \frac{1}{n} E\left(\sum_{i=1}^{n} X_{i-1}\left\{\hat{l}_{i}(t)-i_{n i}(t)\right\}\right)^{2}
\end{aligned}
$$

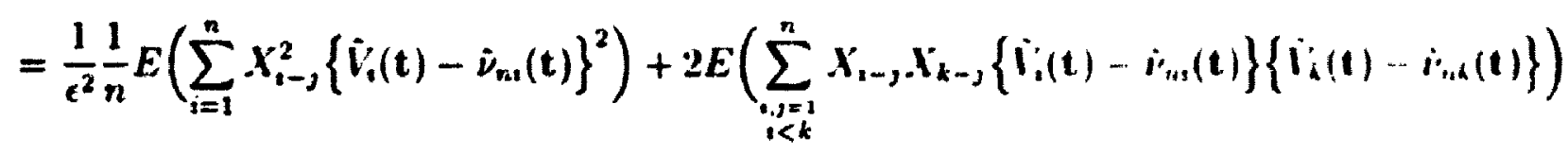

$$
\begin{aligned}
& =\frac{1}{\epsilon^{2}} \frac{1}{n} E\left(\sum_{i=1}^{n} X_{i-j}^{2}\left\{E\left(\tilde{V}_{i}\left(t \mid \mathcal{F}_{i}\right)^{2}-\dot{\nu}_{n k}(t)^{2}\right\}\right)\right. \\
& =\frac{1}{\epsilon^{2}} \frac{1}{n} \sum_{i=1}^{n} E\left(X_{i-j}^{2}\right)\left(\left\{\int_{Q_{0}(\lambda)}^{Q_{0}(\lambda)+n^{-1 / 2} X_{i-1} t} Z^{2} d F_{0}(Z)\right\}-\left\{\int_{Q_{0}(\lambda)}^{Q_{i}(\lambda)+n^{-1 / i X_{i-1}} t / d r_{i}(Z)}\right\}^{2}\right)
\end{aligned}
$$

Now, the first term of R.H.S of (4.63) may be written as

$$
\begin{aligned}
\frac{1}{c^{2}} \frac{1}{n} \sum_{i=1}^{n} E\left(X_{i-j}^{2}\right) & \left\{\int_{Q_{0}(\lambda)}^{Q_{0}(\lambda)+n^{-1 / 2} X_{i-1}^{t} t} Z^{2} d F_{0}(Z) I\left(u^{-1 / 2} X_{i-1}^{\prime} t>\lambda\right)\right. \\
& \left.-\int_{Q_{0}(\lambda)}^{Q_{0}(\lambda)+n^{-1 / 2} X_{i-1} t} Z^{2} d F_{0}(Z) I\left(n^{-1 / 2} X_{i-1}^{\prime} t \leq \delta\right)\right\}
\end{aligned}
$$

$\leq \epsilon$ by al.

By similar argument we get the same result for the second term. Then, we have $J_{1}=o_{P}(1)$ for fixed $t$. For the tightness, we use the compactness property of $\mathcal{N}(b)$. So, it is necessary to show that

$\forall \in>0 \exists \delta>0 \quad \forall \forall s \in \mathcal{N}(b)$

$$
\limsup _{n} P\left(\sup _{\|t-s\| \leq b}\left|T_{n,}^{\infty}(t ; \lambda)-\tilde{T}_{n}^{\infty}(s ; \lambda)\right|>2 \ell\right)<c
$$


In this case, we have

$$
\begin{aligned}
& \left|T_{n j}^{0}(t ; \lambda)-T_{n,}^{0}(s ; \lambda)\right| \leq \\
& \quad n^{-1 / 2} \sum_{i=1}^{n}\left|X_{i-1}, Z_{i}\right|\left|I\left(Z_{i} \leq Q_{0}(\lambda)+n^{-1 / 2} X_{i-1}^{\prime} t\right)-I\left(Z_{i} \leq Q_{0}(\lambda)+n^{-1 / 2} X_{i-1}^{\prime} s\right)\right| \\
& \quad+n^{-1 / 2} \sum_{i=1}^{n}\left|X_{i-j}\right|\left|\mu\left(Q_{0}(\lambda)+n^{-1 / 2} X_{i-1}^{\prime} t\right)-\mu\left(Q_{0}(\lambda)+n^{-1 / 2} X_{i-1}^{\prime} s\right)\right| . \quad
\end{aligned}
$$

Hy the inequality (3.93) the above expression is bounded above by

$$
\begin{aligned}
& n^{-1 / 2} \sum_{i=1}^{n}\left|X_{i-3} Z_{1}\right|\left\{I\left(Z_{1} \leq Q_{0}(\lambda)+n^{-1 / 2} \mathbf{X}_{1-1}^{\prime}+n^{-1 / 2}\left\|\mathbf{X}_{1-1}\right\| \delta\right)\right. \\
& \left.\quad-I\left(Z_{i} \leq Q_{0}(\lambda)+n^{-1 / 2} \mathbf{X}_{i-1}^{\prime}-n^{-1 / 2}\left\|\mathbf{X}_{t-1}\right\| \delta\right)\right\} \\
& +n^{-1 / 2} \sum_{i=1}^{n}\left|X_{i-3}\right|\left|\mu\left(Q_{0}(\lambda)+n^{-1 / 2} \mathbf{X}_{i-1}^{\prime} t\right)-\mu\left(Q_{0}(\lambda)+n^{-1 / 2} \mathbf{X}_{i-1}^{\prime} \delta\right)\right| \\
& =J_{11}+J_{12} .
\end{aligned}
$$

By. mean value Theoren, for sufficiently small $\delta$ and as $n \rightarrow \infty$ we see that sujp $J_{12}=u_{P}(1)$. As we see, $J_{11}$ does not depend on $t$ and so it is enough lo show that $J_{11}=$ op(I) where

$$
\begin{gathered}
J_{11}=n^{-1 / 2} \sum_{i=1}^{n}\left|X_{1-1}\right|\left\{|| Z_{i} \mid I\left(Z_{i} \leq Q_{0}(\lambda)+n^{-1 / 2} \mathbf{X}_{i-1}^{\prime} s+n^{-1 / 2}\left\|\mathbf{X}_{i-1}\right\| \delta\right)\right. \\
\left.-\mu^{-}\left(Q_{0}(\lambda)+n^{-1 / 2} \mathbf{X}_{i-1}^{\prime} s+n^{-1 / 2}\left\|\mathbf{X}_{i-1}\right\| \delta\right)\right] \\
-\left[\left|Z_{i}\right| I\left(Z_{i} \leq Q_{0}(\lambda)+n^{-1 / 2} \mathbf{X}_{i-1}^{\prime}-n^{-1 / 2}\left\|\mathbf{X}_{i-1}\right\| \delta\right)\right. \\
\left.\left.-\mu^{*}\left(Q_{0}(\lambda)+n^{-1 / 2} \mathbf{X}_{i-1}^{\prime}-n^{-1 / 2}\left\|\mathbf{X}_{i-1}\right\| \delta\right)\right]\right\} \\
+n^{-1 / 2} \sum_{i=1}^{n}\left|X_{i-1}\right|\left\{\mu^{-}\left(Q_{0}(\lambda)+n^{-1 / 2} \mathbf{X}_{i-1}^{\prime} s+n^{-1 / 2}\left\|\mathbf{X}_{i-1}\right\| \delta\right)\right. \\
\left.-\mu^{*}\left(Q_{0}(\lambda)+n^{-1 / 2} \mathbf{X}_{i-1}^{\prime} s-n^{-1 / 2}\left\|\mathbf{X}_{i-1}\right\| \delta\right)\right\} \\
=J_{111}+J_{112} \quad \text { respectively. }
\end{gathered}
$$


where

$$
\mu^{*}(x)=E\left(\left|Z_{1}\right| I\left(Z_{1} \leq x\right)\right) \text { and } \mu^{* \prime}(x)=|x| f_{0}(x)
$$

Again, by the mean value Theorem we obtain $J_{112}=o_{r}(1)$. For $I_{111}$. We use the' same idea of proving $J_{1}=o_{P}(1)$ for fixed $t$. Therefore, the proof of I.'mmia 1.1 .1 is complete.

Before we state the next Lemma, it is necessary to introduce the following pro cesses,namely

$$
U_{3 k}(t)=n^{-1} \sum_{i=1}^{n} X_{i-1}, X_{i-d} I\left(Z_{1} \leq Q_{0}(\lambda)+n^{-1 / 2} \mathbf{X}_{i-1}^{\prime} 1\right)
$$

Then we have following lemma.

Lemma 4.4.3 I'nder the conditions of Theore'" 4.4.1. for ach 10 . b. a and $\lambda \in(0,1)$

$$
\sup _{\|t\| \leq b}\left|H_{j k}(t)-\lambda \sigma_{3 k}\right| \stackrel{\prime}{\rightarrow} 0
$$

where $\sigma_{j k}$ is the $j \times k^{\text {th }}$ clement of $\Sigma$.

\section{Proof}

By definition of $U_{j k}(\mathbf{t})$ we have

$$
\begin{aligned}
& \sup _{\|t\| \leq b}\left|U_{\jmath k}(\mathbf{t})-\lambda \sigma_{\jmath k}\right| \\
& =\sup _{\|t\| \leq b}\left|n^{-1} \sum_{i=1}^{n} X_{1-j}, X_{1-k} I\left(Z_{1} \leq Q_{0}(\lambda)+n^{-1 / 2} X_{1-1}^{\prime} t\right)-\sigma_{, k} \lambda\right| \\
& \leq \sup _{\|t\| \leq b}\left|n^{-1} \sum_{i=1}^{n} X_{i-}, X_{i-k}\left\{I\left(Z_{i} \leq Q_{0}(\lambda)+n^{-1 / 2} X_{i-1}^{\prime} t\right)-F_{0}\left(Q_{0}(\lambda)+n^{-1 / 2} X_{i-1}^{\prime} t\right)\right\}\right| \\
& +\sup _{\|t\| \leq b}\left|n^{-1} \sum_{i=1}^{n} X_{i-j} \cdot X_{i-k} F_{0}\left(Q_{0}(\lambda)+n^{-1 / 2} X_{i-1}^{\prime} t\right)-\sigma, k \lambda\right| \\
& =I_{1}+I_{2} \text {. }
\end{aligned}
$$

So, we have to show that $I_{1}=o_{P}(1)=I_{2}$. First, consider $I_{1}$ for fixcod $t \in \mathcal{N}(t)$ ).

$$
I_{1} \leq \mid n^{-1} \sum_{i=1}^{n} X_{i-j} X_{i-k}\left\{I\left(Z_{i} \leq Q_{0}(\lambda)+n^{-1 / 2} X_{i-1}^{\prime} t\right)-r_{i}\left(Q_{0}(\lambda)+n^{-1 / i} X_{i-1}^{\prime} t\right)\right.
$$




$$
\begin{gathered}
-I\left(Z_{1} \leq Q_{0}(\lambda)\right)+F_{0}\left(Q_{0}(\lambda)\right) \mid \\
+\left|n^{-1} \sum_{i=1}^{n} X_{1-j} X_{1-k}\left\{I\left(Z_{1} \leq Q_{0}(\lambda)\right)-F_{0}\left(Q_{0}(\lambda)\right)\right\}\right| \\
=I_{11}+I_{12} .
\end{gathered}
$$

Let us cor:;ider $I_{12}$ first. $I_{12}$ in the absolute value sign is the sum of stationary and ergodic random variables with mean zero and we have

$$
E\left(\left|X_{1-}, X_{i-k}\left\{I\left(Z_{1} \leq Q_{0}(\lambda)\right)-F_{0}\left(Q_{0}(\lambda)\right)\right\}\right|\right) \leq E\left|X_{1-1}, X_{1-k}\right|<x .
$$

'i hen, by Theorem IV.2.2 of Hannan (1970) we have

$$
n^{-1} \sum_{i=1}^{n} X_{i-1} X_{1-k}\left\{I\left(Z_{1} \leq Q_{0}(\lambda)\right)-F_{0}\left(Q_{0}(\lambda)\right)\right\} \rightarrow 0 \text { a.s. }
$$

For $I_{11}$, by the C-S inequality

$$
\begin{aligned}
I_{11}^{2} \leq & n^{-1} \sum_{i=1}^{n} X_{i-1}^{2}, \cdot n^{-1} \sum_{i=1}^{n} X_{i-k}^{2}\left\{I\left(Z_{i} \leq Q_{u}(\lambda)+n^{-1 / 2} \mathbf{X}_{i-1}^{\prime} \mathbf{t}\right)\right. \\
& \left.\quad-F_{0}\left(Q_{0}(\lambda)+n^{-1 / 2} \mathbf{X}_{i-1}^{\prime} \mathbf{t}\right)-I\left(Z_{i} \leq Q_{0}(\lambda)\right)+F_{0}\left(Q_{0}(\lambda)\right)\right\}^{2} \\
= & O_{P}(1) \cdot o_{P}(1) .
\end{aligned}
$$

The first term of R.H.S of $(4.76)$ is $O_{P}(1)$ by B3. The second term is op(1) by Theorem 2.23 of Hall and Heyde (1980). But we have to check the conditions of this theoren. For this reason let us define

$$
\begin{aligned}
l_{n}^{\prime 2}= & \sum_{i=1}^{n}\left(n^{-1 / 2} X_{i-\jmath}\right)^{2}\left\{I\left(Q_{0}(\lambda)<Z_{i} \leq Q_{0}(\lambda)+n^{-1 / 2} \mathbf{X}_{i-1}^{\prime} \mathbf{t}\right)\right. \\
& \left.\quad-F_{0}\left(Q_{0}(\lambda)+n^{-1 / 2} \mathbf{X}_{i-1}^{\prime} \mathbf{t}\right)+F_{0}\left(Q_{0}(\lambda)\right)\right\}^{2} \\
= & \sum_{i=1}^{n} I_{n i}^{2}
\end{aligned}
$$

and

$$
V_{n}^{2}=\sum_{i=1}^{n} E\left(W_{n i}^{2} \mid \mathcal{F}_{i}\right)
$$


The first condition is

$$
\sup _{n} P\left(l_{n}^{-2}>\eta\right) \rightarrow 0 \text { as } \eta \rightarrow \infty
$$

But by definition of $V_{n}^{2}$ we have

$$
\begin{aligned}
V_{n}^{2}= & n^{-1} \sum_{i=1}^{n} X_{i-j}^{2}\left[\left\{F_{0}\left(Q_{0}(\lambda)+n^{-1 / 2} X_{i-1}^{\prime} t\right)-F_{0}\left(Q_{0}(\lambda)\right\}\right.\right. \\
& \times\left(1-\left\{F_{0}\left(Q_{0}(\lambda)+n^{-1 / 2} \mathbf{X}_{i-1}^{\prime} t\right)-F_{0}\left(Q_{0}(\lambda)\right\}\right)\right] \\
\leq & n^{-1} \sum_{i=1}^{n} X_{i-j}^{2}\left\{F_{0}\left(Q_{0}(\lambda)+n^{-1 / 2} X_{i-1}^{\prime} t\right)-F_{0}\left(Q_{u 1}(\lambda)\right\}\right. \\
= & n^{-1} \sum_{i=1}^{n} X_{i-j}^{2}\left(n^{-1 / 2} \mathbf{X}_{t-1}^{\prime} t f_{0}(c)\right) \\
\leq & n^{-1} \sum_{i=1}^{n} X_{i-j}^{2} n^{-1 / 2}\left\|\mathbf{X}_{i-1}\right\| b f_{0}(c) \\
= & O_{P}(1) \cdot o_{P}(1) \cdot b f_{0}(c) \\
= & o_{P}(1) .
\end{aligned}
$$

Therefore, the first condition holds. The second one is the conditional lindelectg, condition, i.e.

$$
\forall \epsilon>0 \quad \sum_{i=1}^{n} E\left\{W_{n}^{2} I\left(\left|W_{n t}\right|>c\right) \mid \mathcal{F}_{1}\right\} \stackrel{\bullet}{\rightarrow} 0
$$

This condition also holds by the definition of $W_{n t}$. Then, by Theorem 2.2:3 of Ilall and Heyde (1980) we have

$$
\left|U_{n}^{2}-V_{n}^{2}\right| \stackrel{P}{\rightarrow} 0
$$

which implies that $I_{1}=o_{P}(1)$. For tightness, we use the compacturns propuerty of $\mathcal{N}(b)$ and nondecreasing property of the indicator function. Then we changer the process with respect to $t$ to a random variable with fixed $s$ and we use a similat method as we did for fixed $t$ in this lemma. As a result we get $I_{1}=0,(1)$.

Now, consider $I_{2}$,

$$
I_{2}=\sup _{\|t\| \leq b}\left|n^{-1} \sum_{i=1}^{n} X_{1-,} X_{1-k} F_{0}\left(Q_{0}(\lambda)+n^{-1 / 2} \mathbf{X}_{i-1}^{\prime} t\right)-\sigma, k \lambda\right|
$$




$$
\begin{aligned}
& \leq \sup _{\|t\| \leq b}\left|n^{-1} \sum_{i=1}^{n} X_{t-j} X_{i-k}\left\{F_{0}\left(Q_{0}(\lambda)+n^{-1 / 2} X_{i-1}^{\prime} t\right)-F_{0}\left(Q_{0}(\lambda)\right)\right\}\right| \\
& \quad+\left|n^{-1} \sum_{i=1}^{n} X_{i-j}, X_{i-k}-\sigma_{j k}\right| F_{0}\left(Q_{0}(\lambda)\right) \\
& =o p(1)+o_{P}(1)=o p(1) \quad \text { by C-S inequality and B3. }
\end{aligned}
$$

Therefore, the proof of Lemma 4.4.3 is complete.

Now we define

$$
U_{j k}(\mathbf{t})=n^{-1} \sum_{i=1}^{n} X_{i-j} X_{i-k} I\left(Q_{0}\left(\lambda_{1}\right)+n^{-1 / 2} \mathbf{X}_{i-1}^{\prime} t<Z_{t} \leq Q_{0}\left(\lambda_{2}\right)+n^{-1 / 2} \mathbf{X}_{i-1}^{\prime} t\right)
$$

for each $\lambda_{1}, \lambda_{2} \ni 0<\lambda_{1}<\lambda_{2}<1$. Then, we have

$$
\sup _{\|t\| \leq b}\left|\ell_{j k}^{\prime *}(t)-\left(\lambda_{2}-\lambda_{1}\right) \sigma_{t k}\right|=o_{P}(1)
$$

Wr. apply this result to prove Theorem 4.4.1

Proof of Theorem 4.4.1

(i): By using the result of Lemma 4.4 .3 in the matrix form we have

$$
\begin{aligned}
& n^{-1} \boldsymbol{X}_{n}^{\prime} \boldsymbol{A} \mathcal{X}_{n}=n^{-1} \sum_{i=1}^{n} \mathbf{X}_{i-1} \boldsymbol{X}_{i-1}^{\prime} I\left(Q_{0}\left(\lambda_{1}\right)+n^{-1 / 2} \mathbf{X}_{i-1}^{\prime} \mathbf{t}<Z_{i}\right. \\
& \left.\leq Q_{0}\left(\lambda_{2}\right)+n^{-1 / 2} \mathbf{X}_{1-1}^{\prime} \mathbf{t}\right) \\
& =\left(\lambda_{2}-\lambda_{1}\right) \boldsymbol{\Sigma}+o_{P}(1) \quad \text { as } n \rightarrow \infty .
\end{aligned}
$$

13y ihe structure of the matrix $A$,

$$
\begin{aligned}
n^{-1 / 2} \boldsymbol{X}_{n}^{\prime} A Z= & n^{-1 / 2} \sum_{i=1}^{n} \mathbf{X}_{1-1}\left\{I\left(Z_{1} \leq Q_{0}\left(\lambda_{2}\right)+\mathbf{X}_{i-1}^{\prime}\left(\hat{\boldsymbol{\rho}}_{n}\left(\lambda_{2}\right)-\rho\left(\lambda_{2}\right)\right)\right)\right. \\
& \left.-I\left(Z_{1} \leq Q_{0}\left(\lambda_{1}\right)+\mathbf{X}_{i-1}^{\prime}\left(\hat{\boldsymbol{\rho}}_{n}\left(\lambda_{1}\right)-\rho\left(\lambda_{1}\right)\right)\right)\right\} Z_{i} \\
= & \tilde{\boldsymbol{T}}_{n}\left(\mathbf{t}_{2} ; \lambda_{2}\right)-\tilde{\boldsymbol{T}}_{n}\left(\mathbf{t}_{1} ; \lambda_{1}\right)
\end{aligned}
$$

where

$$
\mathbf{t}_{1}=n^{1 / 2}\left(\hat{\boldsymbol{\rho}}_{n}\left(\lambda_{\imath}\right)-\boldsymbol{\rho}\left(\lambda_{\imath}\right)\right) \quad i=1,2
$$


But

$$
\begin{aligned}
\dot{\boldsymbol{T}}_{n}\left(t_{2} ; \lambda_{2}\right)-\dot{\boldsymbol{T}}_{n}\left(t_{1} ; \lambda_{1}\right)= & {\left[\dot{\boldsymbol{T}}_{n}\left(t_{2} ; \lambda_{2}\right)-\dot{\boldsymbol{T}}_{n}\left(0 ; \lambda_{2}\right)-q_{0}\left(\lambda_{2}\right) Q_{0}\left(\lambda_{2}\right) \boldsymbol{\Sigma}_{n} t_{2}\right] } \\
& -\left[\dot{T}_{n}\left(t_{1} ; \lambda_{1}\right)-\dot{\boldsymbol{T}}_{n}\left(0 ; \lambda_{1}\right)-q_{0}\left(\lambda_{1}\right) Q_{0}\left(\lambda_{1}\right) \boldsymbol{\Sigma}_{n_{1}} t_{1}\right] \\
& +\left[\dot{\boldsymbol{T}}_{n}\left(0 ; \lambda_{2}\right)-\dot{\boldsymbol{T}}_{n}\left(0 ; \lambda_{1}\right)+q_{0}\left(\lambda_{2}\right) Q_{0}\left(\lambda_{2}\right) \Sigma_{n} t_{2}\right. \\
& \left.-q_{0}\left(\lambda_{1}\right) Q_{0}\left(\lambda_{1}\right) \Sigma_{n} t_{1}\right]
\end{aligned}
$$

By Lemma 4.2.2 the first and second term of (4.89) arc $o_{p}(1)$. Alsu, by substituting $t_{i}$ with its representation given in Theorem 4.2 .1 we have

$$
\begin{aligned}
n^{-1 / 2} X_{n}^{\prime} A Z & =T_{n}\left(\rho\left(\lambda_{1}\right) ; \lambda_{1}\right) Q_{0}\left(\lambda_{1}\right)-T_{n}\left(\rho\left(\lambda_{2}\right) ; \lambda_{2}\right) Q_{0}\left(\lambda_{2}\right) \\
& +n^{-1 / 2} \sum_{i=1}^{n} X_{i-1} Z_{1}\left\{I\left(Q_{0}\left(\lambda_{1}\right)<Z_{1} \leq Q_{u}\left(\lambda_{2}\right)\right)\right\}+u_{1} \cdot(1)
\end{aligned}
$$

and then by definition of $T_{n}(\rho(\lambda): \lambda)$ and using the fact $I\left(Z_{1} \leq a\right)=1-I\left(\%_{1}: a\right)$ we have

$$
\begin{aligned}
n^{-1 / 2} X_{n}^{\prime} A Z= & n^{-1 / 2} \sum_{i=1}^{n} \mathbf{X}_{i-1}^{\prime}\left\{I\left(Z_{1} \leq Q_{0}\left(\lambda_{1}\right)\right) Q_{0}\left(\lambda_{1}\right)+Z_{1} I\left(Q_{0}\left(\lambda_{1}\right)<Z_{2} \leq Q_{0}\left(\lambda_{2}\right)\right)\right. \\
& +I\left(Z_{2}>Q_{0}\left(\lambda_{2}\right)\right) Q_{0}\left(\lambda_{2}\right)-\lambda_{1} Q_{0}\left(\lambda_{1}\right)-\left\{I-\lambda_{2}\right)\left(Q_{0}\left(\lambda_{2}\right)\right\}+(I / 1) \\
= & n^{-1 / 2} \sum_{i=1}^{n} \mathbf{X}_{1-1}\left\{\phi\left(Z_{i}\right)-\gamma\right\}+o_{\mu}(1)
\end{aligned}
$$

where $\phi\left(Z_{i}\right)$ and $\gamma$ are given in (2.97). Finally, we have

$$
\left(\mathcal{X}_{n}^{\prime} A \mathcal{X}_{n}\right)^{-1} \mathcal{X}_{n}^{\prime} A Z=\left(\mathcal{X}_{n}^{\prime} A X_{n}\right)^{-1} \mathcal{X}_{n}^{\prime} A X-\rho
$$

or

$$
\left(n^{-1} \boldsymbol{X}_{n}^{\prime} \boldsymbol{A} \boldsymbol{X}_{n}\right)^{-1} n^{-1 / 2} \boldsymbol{X}_{n}^{\prime} \boldsymbol{A} Z=n^{1 / 2}\left(\bar{\rho}_{n}\left(\lambda_{1}, \lambda_{2}\right)-\rho\right)
$$

and as a result

$$
n^{1 / 2}\left(\bar{\rho}_{n}\left(\lambda_{1}, \lambda_{2}\right)-\rho\right)=\left(\left(\lambda_{2}-\lambda_{1}\right) \Sigma_{n}\right)^{-1} n^{-1 / 2} \sum_{i=1}^{n} \mathbf{X}_{i-1}\left\{\phi\left(Z_{i}\right)-\gamma\right\}+0 \mu(1)
$$


Therefore, the proof of the first part of Theorem 4.4.1 is complete.

Phoof of Theorem 4.4.1

(ii): The result of the second part is obtained by using the result of part (i), taking $F_{0}$ symmetric with $\lambda_{1}=1-\lambda_{2}$ and Corollary 3.1 of Hall and Heyde (1980). For this reason we check the necessary conditions. First, we have to check that

$$
\sum_{i=1}^{n} E\left[n^{-1}\left\|\mathbf{X}_{i-1} \phi\left(Z_{i}\right)\right\|^{2} I\left(n^{-1 / 2}\left\|\mathbf{X}_{i-1} \phi\left(Z_{i}\right)\right\|>\epsilon\right) \mid \mathcal{F}_{i}\right] \rightarrow 0
$$

But.

$$
\begin{aligned}
\left|\phi\left(Z_{1}\right)\right| \leq & \left|I\left(Z_{1} \leq Q_{0}\left(\lambda_{1}\right)\right)\right|\left|Q_{0}\left(\lambda_{1}\right)\right|+\left|I\left(Z_{1} \geq Q_{0}\left(\lambda_{2}\right)\right)\right|\left|Q_{0}\left(\lambda_{2}\right)\right| \\
& +\left|Z_{i} I\left(Q_{0}\left(\lambda_{1}\right)<Z_{i} \leq Q_{0}\left(\lambda_{2}\right)\right)\right|
\end{aligned}
$$

and because $\lambda_{1}$ and $\lambda_{2}$ are fixed, there are $M_{1}, M_{2}>0$ such that $\left|Q_{0}\left(\lambda_{1}\right)\right| \leq M_{1}$ and $\left|Q_{0}\left(\lambda_{2}\right)\right| \leq M_{2}$. Thus, by taking $M=\max \left(M_{1}, M_{2}\right) / 4$ we have

$$
\left|\phi\left(Z_{i}\right)\right| \leq M
$$

and then

$$
\begin{gathered}
n^{-1} \sum_{i=1}^{n} E\left[\left\|\mathrm{X}_{i-1} \phi\left(Z_{i}\right)\right\|^{2} I\left(\left\|\mathrm{X}_{i-1} \phi\left(Z_{i}\right)\right\|>n^{1 / 2} \epsilon\right) \mid \mathcal{F}_{i}\right] \\
\leq M n^{-1} \sum_{i=1}^{n} E\left[\left\|\mathrm{X}_{i-1}\right\|^{2} I\left(\left\|\mathrm{X}_{i-1}\right\|>n^{1 / 2} \epsilon^{*}\right) \mid \mathcal{F}_{i}\right]
\end{gathered}
$$

where $c^{*}=c / M$. Therefore, the first condition is satisfied. For the second condition, we have to show that

$$
\sum_{i=1}^{n} E\left[n^{-1}\left\|\mathbf{X}_{i-1} \phi\left(Z_{i}\right)\right\|^{2} \mid \mathcal{F}_{i}\right] \rightarrow C<\infty
$$

In this case, we have

$$
\begin{aligned}
& n^{-1} \sum_{i=1}^{n} E\left[\left\|\mathbf{X}_{i-1} \phi\left(Z_{i}\right)\right\|^{2} \mid \mathcal{F}_{i}\right] \\
& =n^{-1} \sum_{i=1}^{n}\left\|\mathbf{X}_{i-1}\right\|^{2} E\left(\phi^{2}\left(Z_{i}\right) \mid \mathcal{F}_{i}\right) \\
& \left.=\sigma^{2}\left(\lambda_{1}, \lambda_{2}\right) E\left\|\mathbf{X}_{0}\right\|^{2}\right] \text { as } n \rightarrow \infty
\end{aligned}
$$


Therefore, the proof of Theorem is complete and we obtain

$$
n^{1 / 2}\left(\bar{\rho}_{n}\left(\lambda_{1}, \lambda_{2}\right)-\rho\right) \stackrel{D}{\rightarrow} \mathcal{N}_{p+1}\left(0, \sigma^{2}\left(\lambda_{1}, \lambda_{2}\right) \Sigma^{-1}\right)
$$

\subsection{Estimation of Parameters Under Uncertain Prior Information.}

In the last two sections we estimated the autoregression paramete $\rho$ using $\mathrm{L}$ estimation based on a few selected autoregression quantiles as well as a trimmerl least squares estimator. The estimators could be improved under some prior information on $p$ in the form of a subhypothesis. In this section we are going to improve therse estimators based on some uncertain prior information. We shall obtain the result for a general autoregression quantile for any fixed $\lambda$, which could be replaced ly any of the L-estimators defined in the last sections.

Consider the autoregression model (4.2)

$$
X_{i}=X_{i-1}^{\prime} \rho+Z_{i}, \quad \rho \in \mathbb{R}^{p+1} \quad i=0, \pm 1, \pm 2, \ldots
$$

and let $1 \leq p_{1} \leq p+1$ and $p_{2}=(p+1)-p_{1}$. Partition $p$ into two sulseds namely, $\rho^{\prime}=\left(\rho_{1}^{\prime}, \rho_{2}^{\prime}\right)$ where $\rho_{i} \in \mathbb{R}^{p_{4}} i=1,2$. We are primarily interested in the L-estimation of $\rho_{1}$ when it is suspected but not sure that

$$
H_{0}: \rho_{2}=0
$$

holds. For this we partition the matrix $\boldsymbol{\Sigma}_{n}$ as

$$
\Sigma_{n}=\left[\begin{array}{ll}
\boldsymbol{\Sigma}_{n 11} & \boldsymbol{\Sigma}_{n 12} \\
\boldsymbol{\Sigma}_{n 21} & \boldsymbol{\Sigma}_{n 22}
\end{array}\right]
$$

where $\Sigma_{n 11}, \Sigma_{n 12}$ and $\Sigma_{n 22}$ are $p_{1} \times p_{1}, p_{1} \times p_{2}$ and $p_{2} \times p_{2}$ mat ricen respentively: with $\Sigma_{n 12}=\Sigma_{n 21}^{\prime}$. Now, write $\hat{\rho}_{n}(\lambda)$, the autoregression quantile of $\rho$ for fixed 
$\lambda \in(0,1)$, as $\hat{\boldsymbol{p}}_{n}^{\prime}(\lambda)=\left(\hat{\boldsymbol{\rho}}_{1 n}^{\prime}(\lambda), \hat{\boldsymbol{\rho}}_{2 n}^{\prime}(\lambda)\right)$. In Corollary 4.2.4, we showed that

$$
n^{1 / 2}\left(\hat{\rho}_{n}(\lambda)-\rho(\lambda)\right) \stackrel{D}{\rightarrow} \mathcal{N}_{p+1}\left(0, \frac{\lambda(1-\lambda)}{q_{0}^{2}(\lambda)} \Sigma^{-1}\right) .
$$

Let $\dot{\boldsymbol{\rho}}_{1 n}(\lambda)$ be the restricted L-estimator (RLE) of $\rho_{1}$ while $\dot{\boldsymbol{\rho}}_{1 n}(\lambda)$ be the unrestricted $L$-estimator (ULE) of $\rho_{1}$. This RLE performs better than ULE when $\rho_{2}=0$ holds. We are going to consider the preliminary test L-estimator (PTLE) and the Shrinkage $L$-estimator (SLE) of $\rho_{1}$ when $\rho_{2}$ is suspected to be near 0 . For this reason we need to introduce a test statistic to test $H_{0}: \rho_{2}=0$ as

$$
\mathcal{L}_{n}=n d^{2} \hat{\boldsymbol{\rho}}_{2 n}^{\prime}(\lambda) \Sigma_{n 22.1}^{-1} \dot{\boldsymbol{\rho}}_{2 n}(\lambda)
$$

where

$$
\boldsymbol{\Sigma}_{n 22.1}^{-1}=\boldsymbol{\Sigma}_{n 22}-\boldsymbol{\Sigma}_{n 21} \boldsymbol{\Sigma}_{n 11}^{-1} \boldsymbol{\Sigma}_{n 12} \text { and } d^{2}=\frac{\lambda(1-\lambda)}{q_{0}(\lambda)} .
$$

Then under $H_{0}, \mathcal{L}_{n}$ has asymptotically central chi-square distribution with $p_{2}$ degrees of freedom (df). The PTLE of $\rho_{1}$ is defined by

$$
\dot{\boldsymbol{\rho}}_{1 n}^{P T}(\lambda)=\hat{\boldsymbol{\rho}}_{1 n}(\lambda)+I\left(\mathcal{L}_{n} \geq \gamma_{p_{2}, a}^{2}\right)\left(\hat{\boldsymbol{\rho}}_{1 n}(\lambda)-\dot{\boldsymbol{\rho}}_{1 n}(\lambda)\right)
$$

where $\chi_{p_{2}, \alpha}^{2}$ is the upper $100 \alpha \%$ point of the central chi-squared distribution with $p_{2}$ df. The SLE is defined following Berger, Bock, Brown, Casella and Gleser (197i) as

$$
\dot{\boldsymbol{\rho}}_{i_{n}}^{\mathrm{S}}(\lambda)=\dot{\boldsymbol{\rho}}_{1 n}(\lambda)+\left(\mathbf{I}_{p_{1}}-k c_{n} n^{-1} \mathcal{L}_{n}^{-1} \boldsymbol{W}^{-1} \boldsymbol{\Sigma}_{n 11.2}\right)\left(\dot{\boldsymbol{\rho}}_{1 n}(\lambda)-\dot{\boldsymbol{\rho}}_{1 n}(\lambda)\right)
$$

where $c_{n}=c h_{p_{1}}\left(n \boldsymbol{W} \Sigma_{n 11.2}^{-1}\right)=$ smallest characteristic root of $n \boldsymbol{W} \boldsymbol{\Sigma}_{v .11 .2}^{-1}$, and $k$ is a positive shrinkage factor. If one chooses $W=n^{-1} \Sigma_{n 11.2}$, then we get the simpler form of (4.109)

$$
\tilde{\rho}_{1 n}^{S}(\lambda)=\tilde{\rho}_{1 n}(\lambda)+\left(1-k \mathcal{L}_{n}^{-1}\right)\left(\hat{\rho}_{1 n}(\lambda)-\tilde{\rho}_{1 n}(\lambda)\right), \quad 0<k<2\left(p_{2}-2\right) .
$$

We improve the SLE by defining the positive-rule shrinkage L-estimator (PRSLE) due to Sclove, Morris and Radhakrishnan (1972)

$$
\dot{\boldsymbol{\rho}}_{1 n}^{S+}(\lambda)=\dot{\boldsymbol{\rho}}_{1 n}(\lambda)+\left(1-k \mathcal{L}_{n}^{-1}\right) I\left(\mathcal{C}_{n}>k\right)\left(\hat{\boldsymbol{\rho}}_{1 n}(\lambda)-\dot{\boldsymbol{\rho}}_{1 n}(\lambda)\right)
$$


Note that $\tilde{\rho}_{1 n}^{P T}(\lambda)$ and $\tilde{\rho}_{1 n}^{S+}(\lambda)$ are convex combinations of $\dot{\boldsymbol{p}}_{1 n}(\lambda)$ and $\dot{\boldsymbol{\rho}}_{1 n}(\lambda)$ while $\tilde{\rho}_{1 n}^{S}(\lambda)$ is not. We shall discuss the asymptotic relative optimality of these estimators in the next subsection in terms of asymptotic distribution risk (ADR).

\subsubsection{ADR and ADRE}

We follow Saleh and Sen (1986) and Sen and Saleh (1987) to obtain the expressions for ADR of the above estimators. We use the sequence of alternatives

$$
K_{(n)}: \rho_{2(n)}=n^{-1 / 2} \xi, \quad \xi \in \mathbb{R}^{p^{2}}
$$

Let $H_{m}(\cdot ; \Delta)$ represent the cdf of a noncentral chi-square random varialoke with $m$ df and noncentrality parameter $\Delta$ and

$$
E\left(\chi_{m}^{-2 r}(\Delta)\right)=\int_{0}^{\infty} x^{-r} d H_{m}(x ; \Delta), r \geq 1
$$

For any estimator $\rho_{\text {in }}^{*}$ of $\rho_{1}$ such that

$$
n^{1 / 2}\left(\rho_{i_{n}}^{*}-\rho_{1}\right) \stackrel{\mathcal{D}}{\rightarrow} \mathcal{N}_{p_{1}}\left(0, V^{*}\right)
$$

under $K_{(n)}$, we define the ADR with respect to the loss

$$
n\left(\rho_{i n}^{*}-\rho_{1}\right)^{\prime} W\left(\rho_{i n}^{*}-\rho_{1}\right)
$$

to be

$$
R\left(\rho_{i n}^{*}: W\right) \equiv \operatorname{tr}\left(W V^{*}\right)
$$

where $W$ is a p.d matrix and tr stands for trace operator. The ADR of four estimators are given in the following theorem.

Theorem 4.5.1 Under the conditions al and a.2, the ADR's of the four costimators are given by

$$
R\left(\hat{\rho}_{1 n}(\lambda): W\right)=d^{2} \operatorname{tr}\left(W \Sigma_{11.2}^{-1}\right)
$$




$$
\begin{aligned}
R\left(\tilde{\rho}_{1 n}(\lambda): W\right)= & d^{2} \operatorname{tr}\left(W \Sigma_{11}^{-1}\right)+\xi^{\prime} M \xi \\
R\left(\tilde{\rho}_{1 n}^{P T}(\lambda): W\right)=d^{2}\left\{\operatorname{tr}\left(W \Sigma_{11.2}^{-1}\right)\left[1-H\left(\chi_{p_{2}, a}^{2} ; \Delta\right)\right]\right. & \left.\quad+\operatorname{tr}\left(W \Sigma_{11}^{-1}\right) H_{p_{2}+2}\left(\chi_{p_{2}, a}^{2} ; \Delta\right)\right\} \\
& +\left(\xi^{\prime} M \xi\right)\left[2 H_{p^{2}+2}\left(\chi_{p_{2}, a}^{2} ; \Delta\right)-H_{p_{2}+4}\left(\chi_{p_{2}, a}^{2} ; \Delta\right)\right],( \\
R\left(\tilde{\rho}_{1 n}^{S}(\lambda): W\right)= & d^{2}\left\{\operatorname{tr}\left(W \Sigma_{11.2}^{-1}\right)-k \operatorname{tr}\left(M \Sigma_{22.1}^{-1}\right)\left[2 E\left(\chi_{p_{2}+2}^{-2}(\Delta)\right)\right.\right. \\
& \left.\left.-k E\left(\chi_{p_{2}+2}^{-4}(\Delta)\right)\right]\right\} \\
& +k(k+4)\left(\xi^{\prime} M \xi\right) E\left(\lambda_{p_{2}+4}^{-4}(\Delta)\right)
\end{aligned}
$$

where

$$
M=\Sigma_{21} \Sigma_{11}^{-1} W \Sigma_{11}^{-1} \Sigma_{12}
$$

We shall now discuss the asymptotic distribution risk efficiency (ADRE) results. Note that the risk of $\dot{\boldsymbol{\rho}}_{1 n}(\lambda)$ does not depend on $\xi$ and if we consider the Mahalanobis distance (loss) as

$$
d^{2} n\left(\hat{\rho}_{1 n}(\lambda)-\rho_{1}(\lambda)\right)^{\prime} \Sigma_{11.2}\left(\hat{\rho}_{1 n}(\lambda)-\rho_{1}(\lambda)\right)
$$

then $W=d^{2} \Sigma_{11.2}$, and $R\left(\hat{\rho}_{1 n}(\lambda): W\right)$ reduces to $p_{1}$. Now let

$$
M^{*}=\Sigma_{21} \Sigma_{11}^{-1} \Sigma_{11.2} \Sigma_{11}^{-1} \Sigma_{12} \text { and } M^{0}=\Sigma_{12} \Sigma_{22}^{-1} \Sigma_{21} \Sigma_{11}^{-1}
$$

Theorem 4.5.2 Under the conditions of Theorem 1.5.1, we have

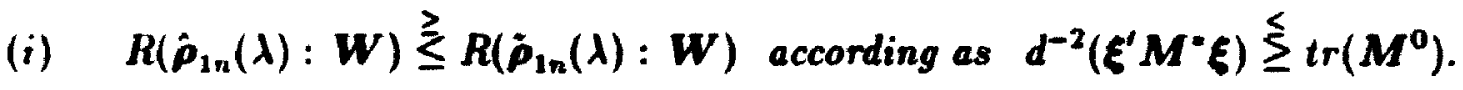

(ii) $\quad R\left(\hat{\boldsymbol{p}}_{1 n}(\lambda): W\right) \stackrel{\gtrless}{ } R\left(\tilde{\rho}_{1 n}^{P T}(\lambda): W\right)$ according as

$$
\left(\xi^{\prime} M^{*} \xi\right) \leqq \frac{d^{2} \operatorname{tr}\left(M^{0}\right) H_{p_{2}+2}\left(\chi_{p_{2}, a}^{2} ; \Delta\right)}{2 H_{p_{2}+2}\left(\chi_{p_{2}, a}^{2} ; \Delta\right)-H_{p_{2}+4}\left(x_{p_{2}, \alpha}^{2} ; \Delta\right)} .
$$

(iii) if $k=\left(p_{2}-2\right)$ and $W$ is an arbitrary positive definite matrix, then

$$
R\left(\tilde{\boldsymbol{\rho}}_{1_{n}}^{S}(\lambda): W\right) \leq R\left(\hat{\boldsymbol{\rho}}_{1_{n}}(\lambda): W\right) \quad \forall W \in \mathcal{W} \text { and } \xi \in \mathbb{R}^{p_{2}}
$$


where

$$
W=\left\{W \text { p.d. }: \frac{\operatorname{tr}\left(M \Sigma_{22.1}^{-1}\right)}{c h_{\max }\left(M \Sigma_{22.1}^{-1}\right)} \geq \frac{p_{2}+2}{2}\right\}
$$

with $M$ given by (4.121) and $\operatorname{ch}_{\max }(A)=$ maximum of the characteristic roots of $A$.

Proof:

The proof of (i) and (ii) is straightforward. We prove (iii). Use the identity

$$
E\left(\chi_{p_{2}+2}^{-2}(\Delta)\right)-\left(p_{2}-2\right) E\left(\chi_{p_{2}+2}^{-4}(\Delta)\right)=\Delta E\left(\gamma_{p_{2}+4}^{-4}(\Delta)\right)
$$

in Theorem 4.5.1 to obtain

$$
\begin{aligned}
& R\left(\dot{\rho}_{1 n}^{S}(\lambda): W\right)=R\left(\dot{\rho}_{1 n}(\lambda): W\right) \\
& -\left(p_{2}-2\right) d^{2} \operatorname{tr}\left(M \Sigma_{22.1}^{-1}\right)\left\{\left(p_{2}-2\right) E\left(x_{p_{2}+2}^{-4}(\Delta)\right)\right. \\
& \left.+\left[1-\frac{\left(p_{2}+2\right)\left(\xi^{\prime} M \xi\right) d^{2}}{2 \operatorname{tr}\left(M \Sigma_{22.1}^{-1}\right) \Delta}\right] 2 \Delta E\left(x_{r_{2}+4}^{-4}(\Delta)\right)\right\}
\end{aligned}
$$

From (1.126), it follows that

$$
R\left(\hat{\boldsymbol{\rho}}_{1 n}^{S}(\lambda): \boldsymbol{W}\right) \leq R\left(\dot{\boldsymbol{\rho}}_{1 n}(\lambda): \boldsymbol{W}\right)
$$

for all $\varepsilon \in \mathbb{R}^{p 2}$ if

$$
\frac{\operatorname{tr}\left(M \Sigma_{22.1}^{-1}\right)}{c h_{\max }\left(M \Sigma_{22.1}^{-1}\right)} \geq \frac{\left(p_{2}+2\right)}{2}
$$

that is, if $\boldsymbol{W} \in \boldsymbol{W}$.

In particular, if $\boldsymbol{W}=d^{2} \Sigma_{11.2}$, then the risk expression (4.127) is the sante with $M$ replaced by $M^{*}$ of $(4.123)$. In this case the condition (4.125) reduces $t, 1$,

$$
W=\left\{W \text { p.d. }: \frac{\operatorname{tr}\left(M^{0}\right)}{c h_{\max }\left(M^{0}\right)} \geq \frac{p_{2}+2}{2}\right\}
$$

since

$$
\operatorname{tr}\left(M^{*} \Sigma_{22.1}^{-1}\right)=\operatorname{tr}\left(M^{0}\right)
$$

We note that $\operatorname{tr}\left(M^{0}\right)=\operatorname{tr}\left(I_{p_{1}}-\Sigma_{11.2} \Sigma_{11}^{-1}\right) \leq \min \left(p_{1}, p_{2}\right)=p^{*}>3$ simc $\cdots p_{2}>3$. Also, if the rank of $M^{0} \leq 2$, then (4.129) does not hold with $M=M^{\circ}$. However. 
if $p_{2} \geq 3$ and $2 \operatorname{tr}\left(M^{0}\right) \geq c h_{\max }\left(M^{0}\right)$, then (4.129) holds. Thus, for Mahalanobis loss, (iii) holds. Fortunately, the condition (4.129) is verifiable for a given $\Sigma_{n}$ for any $\mathbf{A R}(\mathbf{p})$ model. For an arbitrary $\boldsymbol{W}$ not belonging to $W$, (iii) may not hold for all $M$. In that case one may use the SLE given in (4.110).

Now we assume that $k$ is an arbitrary positive integer and that $W=d^{-2} \Sigma_{11.2}$. The following theorem gives a sufficient condition on $k$ for which SLE dominates ULE under ADR.

Theorem 4.5.3 A sufficient condition for the asymptotic dominance of SLE over $U L E\left[\right.$ i.e., $\left.R\left(\hat{\boldsymbol{\rho}}_{1 n}(\lambda): W\right) \geq R\left(\hat{\boldsymbol{\rho}}_{1 n}^{S}(\lambda): W\right) \forall \xi \in \mathbb{E}^{p_{2}}\right]$ is that the shrinkage factor $k$ is positive and satisfies the inequality

$$
2 E\left(x_{p_{2}+2}^{-2}(\Delta)\right)-k E\left(x_{p_{2}+2}^{-4}(\Delta)\right)-(k+4) h \Delta E\left(x_{p_{2}+2}^{-4}(\Delta)\right) \geq 0 \quad \forall \Delta,
$$

which in turn, requires that

$$
p_{2} \geq 3, \quad 0<k<2\left(p_{2}-2\right) \text { and } h(k+4) \leq 2
$$

where $h=c h_{\max }\left(M^{0}\right) / \operatorname{tr}\left(M^{0}\right)$ so that $0<h<1$.

\section{Proof:}

Details of the proof are similar to Sen and Saleh (1987) and are left out.

Similarly, one has the following theorem.

Theorem 4.3.4 Under the conditions of Theorem 4.5.3, the PTLE fails to dominate SLE. Also, if for an $\alpha(0<\alpha<1)$, the level of significance of the $P T$, we have

$$
\begin{gathered}
H_{p_{2}+2}\left(\chi_{p_{2}, \alpha}^{2} ; 0\right) \geq q\left\{2\left(p_{2}-2\right)-q\right\} / p_{2}\left(p_{2}-2\right), \\
q=\left(p_{2}-2\right) \wedge(2 / h-4) .
\end{gathered}
$$

then the SLE fails to dominate the PTLE. 
However, one may verify that under $H_{0}$ the ordering of the ADR of the four extimators is as follows:

$$
R\left(\dot{\boldsymbol{\rho}}_{1 n}(\lambda): W\right) \leq R\left(\dot{\boldsymbol{\rho}}_{1 n}^{K T}(\lambda): W\right) \leq R\left(\hat{\boldsymbol{\rho}}_{1 n}^{S}(\lambda): W\right) \leq R\left(\dot{\rho}_{1 n}(\lambda): W^{\prime}\right)
$$

The general behavior of these risks is similar to those discussed in Sin and Saleht (1987) in connection with linear regression models. 


\section{Appendix A}

\section{t-dist. (location and scale parameters)}


Table A.1: Optimal Spacing for the location and scale parameters along with the coefficeints for the ABLUE of t-distribution.

\begin{tabular}{|c|c|c|c|c|c|c|c|c|c|}
\hline \multicolumn{10}{|c|}{ D.F. $=1$} \\
\hline$k$ & 2 & 3 & 4 & 5 & 6 & $i$ & 8 & $y$ & 10 \\
\hline $\begin{array}{l}\lambda_{1} \\
a_{1} \\
b_{1}\end{array}$ & $\begin{array}{r}0.3333 \\
0.5000 \\
-.8660\end{array}$ & $\begin{array}{r}0.2500 \\
0.0000 \\
-.5000\end{array}$ & $\begin{array}{r}0.2000 \\
-.0854 \\
-.2629\end{array}$ & $\begin{array}{r}0.1667 \\
-.0833 \\
-.1443\end{array}$ & $\begin{array}{r}0.1429 \\
-.0671 \\
-.0841\end{array}$ & $\begin{array}{c}0.1250 \\
-.0518 \\
-.0518\end{array}$ & $\begin{array}{c}0.1111 \\
-.0398 \\
-.0331\end{array}$ & $\begin{array}{r}0.1000 \\
-.030 ! \\
-.0225\end{array}$ & $\begin{array}{l}0.0941 ! ! \\
-.01913 \\
-.01: 46\end{array}$ \\
\hline $\begin{array}{l}\lambda_{2} \\
a_{2} \\
b_{2}\end{array}$ & $\begin{array}{l}0.6667 \\
0.5000 \\
0.8660\end{array}$ & $\begin{array}{l}0.5000 \\
1.0000 \\
0.0000\end{array}$ & $\begin{array}{c}0.4000 \\
0.5854 \\
-.4253\end{array}$ & $\begin{array}{c}0.3333 \\
0.2500 \\
-.4330\end{array}$ & $\begin{array}{r}0.2857 \\
0.0777 \\
-.3405\end{array}$ & $\begin{array}{r}0.2500 \\
0.0000 \\
-.2500\end{array}$ & $\begin{array}{r}0.2223 \\
-.03119 \\
-.1808\end{array}$ & $\begin{array}{l}0.2010) \\
-.01: 27 \\
-.131 .1\end{array}$ & $\begin{array}{l}0.1818 \\
-.011: \\
-.04015\end{array}$ \\
\hline $\begin{array}{l}\lambda_{3} \\
a_{3} \\
b_{3}\end{array}$ & & $\begin{array}{l}0.7500 \\
0.0000 \\
0.5000\end{array}$ & $\begin{array}{l}0.6000 \\
0.5854 \\
0.4253\end{array}$ & $\begin{array}{l}0.5000 \\
0.6667 \\
0.0000\end{array}$ & $\begin{array}{c}0.4286 \\
0.4893 \\
-.235 \bar{T}\end{array}$ & $\begin{array}{c}0.3750 \\
0.3018 \\
-.3018\end{array}$ & $\begin{array}{l}0.333333 \\
0.1667 \\
-.2887\end{array}$ & $\begin{array}{l}0.30(1) \\
0.0 \times(1) ! \\
-.21401\end{array}$ & 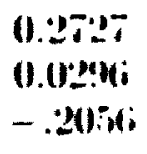 \\
\hline $\begin{array}{l}\lambda_{4} \\
a_{4} \\
b_{4}\end{array}$ & & & $\begin{array}{c}0.8000 \\
-.0854 \\
0.2629\end{array}$ & $\begin{array}{l}0.6667 \\
0.2500 \\
0.4330\end{array}$ & $\begin{array}{l}0.5714 \\
0.4893 \\
0.2357\end{array}$ & $\begin{array}{l}0.5000 \\
0.5000 \\
0.0000\end{array}$ & $\begin{array}{r}0.1111 \\
0.1050 \\
-.147 .1\end{array}$ & $\begin{array}{l}0.101101 \\
0.29127 \\
-.2127\end{array}$ & $\begin{array}{l}\text { 0.3tikfi } \\
\text { 0.11110 } \\
-.227 .1\end{array}$ \\
\hline $\begin{array}{l}\lambda_{5} \\
a_{5} \\
b_{5}\end{array}$ & & & & $\begin{array}{c}0.8333 \\
-.0833 \\
0.1443\end{array}$ & $\begin{array}{l}0.7143 \\
0.0777 \\
0.3405\end{array}$ & $\begin{array}{l}0.6250 \\
0.3018 \\
0.3018\end{array}$ & $\begin{array}{l}0.55 .56 \\
0.4050 \\
0.1471\end{array}$ & $\begin{array}{l}0.50(1) 0 \\
0.100(0) \\
0.0(0)(1)\end{array}$ & $\begin{array}{l}0.4515 \\
0.311 \times \\
-.|(n)|\end{array}$ \\
\hline $\begin{array}{l}\lambda_{6} \\
a_{6} \\
b_{i}\end{array}$ & & & & & $\begin{array}{c}0.8571 \\
-.0671 \\
0.0841\end{array}$ & $\begin{array}{l}0.7500 \\
0.0000 \\
0.2500\end{array}$ & $\begin{array}{l}0.66675 \\
0.1667 \\
0.2 \times \times 7\end{array}$ & $\begin{array}{l}0 .(a)(x) \\
0.29+27 \\
0.21 \cdot 27\end{array}$ & $\begin{array}{l}0.515 ; \\
0.311 \mathrm{~s} \\
0.1(\mathrm{M}) \mathrm{l}\end{array}$ \\
\hline $\begin{array}{l}\lambda_{7} \\
a_{7} \\
b_{7}\end{array}$ & & & & & & $\begin{array}{c}0.8750 \\
-.0518 \\
0.0518\end{array}$ & $\begin{array}{c}0.7778 \\
-.0319 \\
0.1808\end{array}$ & $\begin{array}{l}0.7001) \\
0.08(19) \\
0.2490\end{array}$ & $\begin{array}{l}0.636 i 1 \\
0.1970 \\
0.2+27.1\end{array}$ \\
\hline $\begin{array}{l}\lambda_{8} \\
a_{8} \\
b_{8}\end{array}$ & & & & & & & $\begin{array}{c}0.8889 \\
-.039 \times \\
0.033341\end{array}$ & $\begin{array}{c}0.8000 \\
-.0127 \\
0.1: 114\end{array}$ & $\begin{array}{l}0.727: 3 \\
0.012 ! \mathrm{k}, \\
0.211 . x,\end{array}$ \\
\hline $\begin{array}{l}\lambda_{9} \\
a_{9} \\
b_{9}\end{array}$ & & & & & & & & $\begin{array}{c}0.90(0) \\
-.0309 \\
0.0 .2 \times)\end{array}$ & $\begin{array}{l}0 . \times 1 \times 2 \\
-.0112 \\
0.0 \times 4,1\end{array}$ \\
\hline $\begin{array}{l}\lambda_{10} \\
a_{10} \\
b_{10}\end{array}$ & & & & & & & & & $\begin{array}{l}0 .(x)(1) \\
-.029 .8 \\
0 .(11: x\end{array}$ \\
\hline $\begin{array}{c}\boldsymbol{K}_{1} \\
\boldsymbol{K}_{2} \\
\boldsymbol{K}_{3} \\
\Delta\end{array}$ & $\begin{array}{l}0.3420 \\
0.3420 \\
0.0000 \\
0.1169\end{array}$ & $\begin{array}{l}0.405 .3 \\
0.4053 \\
0.0000 \\
0.1643\end{array}$ & $\begin{array}{l}0.4376 \\
0.4376 \\
0.0000 \\
0.1915\end{array}$ & $\begin{array}{l}0.4559 \\
0.4559 \\
0.0000 \\
0.2079\end{array}$ & $\begin{array}{l}0.4673 \\
0.4673 \\
0.0000 \\
0.2184\end{array}$ & $\begin{array}{l}0.4748 \\
0.4748 \\
0.0000 \\
0.225 .5\end{array}$ & $\begin{array}{l}0.1800 \\
0.4800 \\
0.00(10) \\
0.2304\end{array}$ & $\begin{array}{l}0.45 .3 \times \\
0.48 .3 \times \\
0.00(301 \\
0.23 .40\end{array}$ & $\begin{array}{l}0.450,6 \\
0.456,6 \\
0.013111 \\
0.2367\end{array}$ \\
\hline
\end{tabular}


Table: A.2: Optimal Spacing for the location and scale parameters along with the roefficeints for the ABLUE of t-distribution.

$$
\text { D.F. }=2
$$

\begin{tabular}{|c|c|c|c|c|c|c|c|c|c|}
\hline$k$ & 2 & 3 & 4 & 5 & 6 & 7 & 8 & 9 & 10 \\
\hline $\begin{array}{l}\lambda_{1} \\
a_{1} \\
b_{1}\end{array}$ & $\begin{array}{r}0.2688 \\
0.5000 \\
-.6778\end{array}$ & $\begin{array}{c}0.1964 \\
0.1091 \\
-.4627\end{array}$ & $\begin{array}{l}0.1487 \\
-.0037 \\
-.2674\end{array}$ & $\begin{array}{l}0.1183 \\
-.0312 \\
-.1662\end{array}$ & $\begin{array}{r}0.0968 \\
-.0355 \\
-.1078\end{array}$ & $\begin{array}{l}0.0812 \\
-.0329 \\
-.0731\end{array}$ & $\begin{array}{l}0.0693 \\
-.0286 \\
-.0313\end{array}$ & $\begin{array}{l}0.0601 \\
-.0244 \\
-.03 i 1\end{array}$ & $\begin{array}{l}0.052 i \\
-.0206 \\
-.0275\end{array}$ \\
\hline $\begin{array}{l}\lambda_{2} \\
a_{2} \\
b_{2}\end{array}$ & $\begin{array}{l}0.7312 \\
0.5000 \\
0.6778\end{array}$ & $\begin{array}{l}0.5000 \\
0.7819 \\
0.0000\end{array}$ & $\begin{array}{l}0.3634 \\
0.5037 \\
-.3154\end{array}$ & $\begin{array}{l}0.2854 \\
0.2578 \\
-.3306\end{array}$ & $\begin{array}{l}0.2327 \\
0.1216 \\
-.2713\end{array}$ & $\begin{array}{l}0.1951 \\
0.0523 \\
-.2109\end{array}$ & $\begin{array}{l}0.1669 \\
0.0168 \\
-.1625\end{array}$ & $\begin{array}{l}0.1451 \\
-.0014 \\
-.1259\end{array}$ & $\begin{array}{r}0.1278 \\
-.0106 \\
-.0986\end{array}$ \\
\hline $\begin{array}{l}\lambda_{3} \\
a_{3} \\
b_{3}\end{array}$ & & $\begin{array}{l}0.8036 \\
0.1091 \\
0.462 \tau\end{array}$ & $\begin{array}{l}0.6366 \\
0.5037 \\
0.3154\end{array}$ & $\begin{array}{l}0.5000 \\
0.5467 \\
0.0000\end{array}$ & $\begin{array}{l}0.4028 \\
0.4139 \\
-.1760\end{array}$ & $\begin{array}{c}0.3349 \\
0.2718 \\
-.2264\end{array}$ & $\begin{array}{l}0.2850 \\
0.1690 \\
-.2191\end{array}$ & $\begin{array}{c}0.24 i 1 \\
0.102 .3 \\
-.193: 1\end{array}$ & $\begin{array}{l}0.2172 \\
0.0600 \\
-.16 .17\end{array}$ \\
\hline $\begin{array}{l}\lambda_{4} \\
a_{4}\end{array}$ & & & $\begin{array}{c}0.8813 \\
-.003 i \\
0.2674\end{array}$ & $\begin{array}{l}0.7146 \\
0.2578 \\
0.3306\end{array}$ & $\begin{array}{l}0.5972 \\
0.4139 \\
0.1760\end{array}$ & $\begin{array}{l}0.5000 \\
0.4175 \\
0.0000\end{array}$ & $\begin{array}{c}0.4243 \\
0.3428 \\
-.1119\end{array}$ & $\begin{array}{r}0.3661 \\
0.2548 \\
-.1608\end{array}$ & $\begin{array}{l}0.3206 \\
0.1809 \\
-.1717\end{array}$ \\
\hline$\lambda_{5}$ & & & & $\begin{array}{c}0.8817 \\
-.0312 \\
0.1662\end{array}$ & $\begin{array}{l}0.7673 \\
0.12 i 6 \\
0.2713\end{array}$ & $\begin{array}{l}0.6651 \\
0.2718 \\
0.2264\end{array}$ & $\begin{array}{l}0.5757 \\
0.3428 \\
0.1119\end{array}$ & $\begin{array}{l}0.5000 \\
0.3373 \\
0.0000\end{array}$ & $\begin{array}{l}0.1379 \\
0.2902 \\
-.0773\end{array}$ \\
\hline $\begin{array}{l}\lambda_{6 ;} \\
a_{6} \\
b_{6}\end{array}$ & & & & & $\begin{array}{c}0.9032 \\
-.0355 \\
0.1078\end{array}$ & $\begin{array}{l}0.8049 \\
0.0523 \\
0.2109\end{array}$ & $\begin{array}{l}0.7150 \\
0.1690 \\
0.2191\end{array}$ & $\begin{array}{l}0.6339 \\
0.2518 \\
0.1608\end{array}$ & $\begin{array}{l}0.5621 \\
0.2902 \\
0.0773\end{array}$ \\
\hline $\begin{array}{l}\lambda_{i} \\
a_{7} \\
b_{7}\end{array}$ & & & & & & $\begin{array}{c}0.9188 \\
-.0329 \\
0.0731\end{array}$ & $\begin{array}{l}0.8331 \\
0.0168 \\
0.1625\end{array}$ & $\begin{array}{l}0.7529 \\
0.1023 \\
0.1933\end{array}$ & $\begin{array}{l}0.6794 \\
0.1809 \\
0.1717\end{array}$ \\
\hline $\begin{array}{l}\lambda_{8} \\
a_{k} \\
b_{s}\end{array}$ & & & & & & & $\begin{array}{c}0.9307 \\
-.0286 \\
0.0513\end{array}$ & $\begin{array}{c}0.8549 \\
-.0014 \\
0.1259\end{array}$ & $\begin{array}{l}0.7828 \\
0.0600 \\
0.1647\end{array}$ \\
\hline $\begin{array}{l}\lambda_{1} \\
a_{9} \\
b_{3}\end{array}$ & & & & & & & & $\begin{array}{c}0.9399 \\
-.0244 \\
0.0371\end{array}$ & $\begin{array}{c}0.8722 \\
-.0106 \\
0.0986\end{array}$ \\
\hline $\begin{array}{l}\lambda_{10} \\
a_{10} \\
b_{10}\end{array}$ & & & & & & & & & $\begin{array}{c}0.9473 \\
-.0206 \\
0.0275\end{array}$ \\
\hline $\begin{array}{l}\boldsymbol{K}_{1} \\
\boldsymbol{K}_{2} \\
\boldsymbol{K}_{3} \\
\dot{J}\end{array}$ & $\begin{array}{l}0.4519 \\
0.5317 \\
0.0000 \\
0.2403\end{array}$ & $\begin{array}{l}0.5248 \\
0.6161 \\
0.0000 \\
0.3233\end{array}$ & $\begin{array}{l}0.5452 \\
0.6842 \\
0.0000 \\
0.3730\end{array}$ & $\begin{array}{l}0.5609 \\
0.7174 \\
0.0000 \\
0.4024\end{array}$ & $\begin{array}{l}0.5702 \\
0.7389 \\
0.0000 \\
0.4214\end{array}$ & $\begin{array}{l}0.5767 \\
0.7528 \\
0.0000 \\
0.4342\end{array}$ & $\begin{array}{l}0.5813 \\
0.7625 \\
0.0000 \\
0.4433\end{array}$ & $\begin{array}{l}0.5847 \\
0.7695 \\
0.0000 \\
0.4499\end{array}$ & $\begin{array}{l}0.5872 \\
0.7746 \\
0.0000 \\
0.4549\end{array}$ \\
\hline
\end{tabular}


Table A.3: Optimal Spacing for the location and scale parameters along with the coefficeints for the ABLUE of $t$-distribution.

\begin{tabular}{|c|c|c|c|c|c|c|c|c|c|}
\hline \multicolumn{10}{|c|}{ D.F. $=3$} \\
\hline$k$ & 2 & 3 & 4 & 5 & 6 & 7 & 8 & 9 & 10 \\
\hline $\begin{array}{l}\lambda_{1} \\
a_{1} \\
b_{1}\end{array}$ & $\begin{array}{l}0.2352 \\
0.5000 \\
-.6069\end{array}$ & $\begin{array}{l}0.1672 \\
0.1467 \\
-.4358\end{array}$ & $\begin{array}{l}0.1212 \\
0.0322 \\
-.2554\end{array}$ & $\begin{array}{l}0.0939 \\
-.0018 \\
-.1657\end{array}$ & $\begin{array}{r}0.0750 \\
-.0134 \\
-.1115\end{array}$ & $\begin{array}{r}0.0616 \\
-.0165 \\
-.0787\end{array}$ & $\begin{array}{l}0.0516 \\
-.0165 \\
-.0573\end{array}$ & $\begin{array}{r}0.0440 \\
-.0153 \\
-.0429 !\end{array}$ & $\begin{array}{l}0.0381 \\
-.01: 28 \\
-.032 ! 9\end{array}$ \\
\hline $\begin{array}{l}\lambda_{2} \\
a_{2} \\
b_{2}\end{array}$ & $\begin{array}{l}0.7648 \\
0.5000 \\
0.6069\end{array}$ & $\begin{array}{l}0.5000 \\
0.7066 \\
0.0000\end{array}$ & $\begin{array}{l}0.3405 \\
0.4678 \\
-.2844\end{array}$ & $\begin{array}{l}0.2579 \\
0.2520 \\
-.2974\end{array}$ & $\begin{array}{l}0.2035 \\
0.1311 \\
-.2444\end{array}$ & $\begin{array}{l}0.1662 \\
0.0687 \\
-.1921\end{array}$ & $\begin{array}{l}0.1390 \\
0.0349 \\
-.150 .4\end{array}$ & $\begin{array}{l}0.1184 \\
0.0161 \\
-.1187\end{array}$ & $\begin{array}{l}0.1112 .4 \\
0.0055 .3 \\
-.0914\end{array}$ \\
\hline $\begin{array}{l}\lambda_{3} \\
a_{3} \\
b_{3}\end{array}$ & & $\begin{array}{l}0.8328 \\
0.1467 \\
0.4358\end{array}$ & $\begin{array}{l}0.6595 \\
0.4678 \\
0.2844\end{array}$ & $\begin{array}{l}0.5000 \\
0.4995 \\
0.0000\end{array}$ & $\begin{array}{l}0.386 i \\
0.3823 \\
-.1593\end{array}$ & $\begin{array}{l}0.3114 \\
0.2557 \\
-.2033\end{array}$ & $\begin{array}{l}0.2579 \\
0.1648 \\
--.1957\end{array}$ & $\begin{array}{l}0.2181 \\
0.1062 \\
-.1728\end{array}$ & $\begin{array}{l}0.15 \times 1 \\
0.06 i s t \\
-.1 .4 s 1\end{array}$ \\
\hline $\begin{array}{l}\lambda_{4} \\
a_{4} \\
b_{4}\end{array}$ & & & $\begin{array}{l}0.8788 \\
0.0322 \\
0.2554\end{array}$ & $\begin{array}{l}0.7421 \\
0.2520 \\
0.2974\end{array}$ & $\begin{array}{l}0.6133 \\
0.3823 \\
0.1593\end{array}$ & $\begin{array}{l}0.5000 \\
0.3543 \\
0.0000\end{array}$ & $\begin{array}{l}0.4118 \\
0.3168 \\
-.1017\end{array}$ & $\begin{array}{l}0.3461 \\
0.2372 \\
-.1450\end{array}$ & $\begin{array}{l}0.29(61) \\
0.1713 \\
-.1535\end{array}$ \\
\hline $\begin{array}{l}\lambda_{5} \\
a_{5} \\
b_{5}\end{array}$ & & & & $\begin{array}{c}0.9061 \\
-.0018 \\
0.1657\end{array}$ & $\begin{array}{l}0.7965 \\
0.1311 \\
0.2444\end{array}$ & $\begin{array}{l}0.6886 \\
0.2557 \\
0.2033\end{array}$ & & & $\begin{array}{l}0.4277 \\
0.2686 \\
-.0705\end{array}$ \\
\hline $\begin{array}{l}\lambda_{6} \\
a_{6} \\
b_{6}\end{array}$ & & & & & $\begin{array}{c}0.9250 \\
-.0134 \\
0.1115\end{array}$ & $\begin{array}{l}0.8338 \\
0.0687 \\
0.1921\end{array}$ & $\begin{array}{l}0.7421 \\
0.1648 \\
0.1958\end{array}$ & $\begin{array}{l}0.6539 \\
0.2372 \\
0.1 .150\end{array}$ & $\begin{array}{l}0.572 .3 \\
0.26 \times 6 \\
0.0705\end{array}$ \\
\hline $\begin{array}{l}\lambda_{7} \\
a_{7} \\
b_{7}\end{array}$ & & & & & & $\begin{array}{c}0.9384 \\
-.016 .5 \\
0.0787\end{array}$ & $\begin{array}{l}0.8610 \\
0.0349 \\
0.150 .1\end{array}$ & & $\begin{array}{l}0.70 .39 \\
0.1713 \\
0.15 .37\end{array}$ \\
\hline $\begin{array}{l}\lambda_{8} \\
a_{8} \\
b_{8}\end{array}$ & & & & & & & $\begin{array}{c}0.9484 \\
-.016 .7 \\
0.0573\end{array}$ & $\begin{array}{l}0.8816 \\
0.0161 \\
0.1187\end{array}$ & $\begin{array}{l}0.8119 \\
0.0686 ; \\
0.14 \times 1\end{array}$ \\
\hline $\begin{array}{l}\lambda_{9} \\
a_{9} \\
b_{9}\end{array}$ & & & & & & & & $\begin{array}{c}0.95(60) \\
-.015 .3 \\
0.0429\end{array}$ & $\begin{array}{l}0.8976 \\
0 .(00) \\
0.0948\end{array}$ \\
\hline $\begin{array}{l}\lambda_{10} \\
a_{10} \\
b_{10}\end{array}$ & & & & & & & & & $\begin{array}{r}0.96119 \\
-.013 \times \\
0.0329\end{array}$ \\
\hline $\begin{array}{c}K_{1} \\
K_{2} \\
K_{3} \\
\Delta\end{array}$ & $\begin{array}{l}0.5081 \\
0.6511 \\
0.0000 \\
0.3308\end{array}$ & $\begin{array}{l}0.5940 \\
0.7457 \\
0.0000 \\
0.4430\end{array}$ & $\begin{array}{l}0.6124 \\
0.8395 \\
0.0000 \\
0.5141\end{array}$ & $\begin{array}{l}0.6292 \\
0.8831 \\
0.0000 \\
0.5556\end{array}$ & $\begin{array}{l}0.6379 \\
0.9134 \\
0.0000 \\
0.5826\end{array}$ & $\begin{array}{l}0.6442 \\
0.9327 \\
0.0000 \\
0.6009\end{array}$ & $\begin{array}{l}0.6486 \\
0.9465 \\
0.0000 \\
0.6138\end{array}$ & $\begin{array}{l}0.65 .518 \\
0.956 .3 \\
0.00(40) \\
0.6 ; 23.3\end{array}$ & $\begin{array}{l}0.6512 \\
0.964 .37 \\
0.0000) \\
0(630)\end{array}$ \\
\hline
\end{tabular}


Table A.4: Optimal Spacing for the location and scale parameters along with the coefficeints for the ABLUE of $t$-distribution.

$$
\text { D.F. }=5
$$

\begin{tabular}{|c|c|c|c|c|c|c|c|c|c|}
\hline$k$ & 2 & 3 & 4 & 5 & 6 & 7 & 8 & 9 & 10 \\
\hline $\begin{array}{l}\lambda_{1} \\
a_{3} \\
b_{1}\end{array}$ & $\begin{array}{c}0.2012 \\
0.5000 \\
-.5467\end{array}$ & $\begin{array}{r}0.1380 \\
0.1770 \\
-.4089\end{array}$ & $\begin{array}{r}0.0936 \\
0.0623 \\
-.2386\end{array}$ & & & & & $\begin{array}{r}0.0290 \\
-.0041 \\
-.0449\end{array}$ & $\begin{array}{r}0.0246 \\
-.0046 \\
-.0352\end{array}$ \\
\hline $\begin{array}{l}\lambda_{2} \\
a_{2} \\
b_{2}\end{array}$ & $\begin{array}{l}0.7988 \\
0.5000 \\
0.5467\end{array}$ & $\begin{array}{l}0.5000 \\
0.6459 \\
0.0000\end{array}$ & $\begin{array}{l}0.3134 \\
0.4377 \\
-.2619\end{array}$ & $\begin{array}{l}0.2275 \\
0.2450 \\
-.2724\end{array}$ & $\begin{array}{l}0.1715 \\
0.135 ! \\
-.2221\end{array}$ & & & $\begin{array}{r}0.0907 \\
-.0298 \\
-.1099\end{array}$ & $\begin{array}{l}0.0764 \\
-.0184 \\
-.0886\end{array}$ \\
\hline $\begin{array}{l}\lambda_{3} \\
a_{3} \\
b_{3}\end{array}$ & & $\begin{array}{l}0.8620 \\
0.1770 \\
0.4089\end{array}$ & $\begin{array}{l}0.6866 \\
0.4377 \\
0.2619\end{array}$ & $\begin{array}{l}0.5000 \\
0.4604 \\
0.0000\end{array}$ & & & & $\begin{array}{l}0.1863 \\
0.1072 \\
-.1566\end{array}$ & $\begin{array}{l}0.1561 \\
0.0737 \\
-.1341\end{array}$ \\
\hline $\begin{array}{l}\lambda_{4} \\
a_{4} \\
b_{4}\end{array}$ & & & $\begin{array}{l}0.9064 \\
0.0623 \\
0.2386\end{array}$ & $\begin{array}{l}0.7725 \\
0.2450 \\
0.2724\end{array}$ & & & & $\begin{array}{l}0.3220 \\
0.2217 \\
-.1346\end{array}$ & $\begin{array}{l}0.2674 \\
0.1617 \\
-.1409\end{array}$ \\
\hline $\begin{array}{l}\lambda_{5} \\
a_{5} \\
b_{5}\end{array}$ & & & & $\begin{array}{l}0.9301 \\
0.0248 \\
0.1594\end{array}$ & & & & & \\
\hline $\begin{array}{l}\lambda_{6} \\
a_{6} \\
b_{6}\end{array}$ & & & & & & & & & $\begin{array}{l}0.5851 \\
0.2508 \\
0.0667\end{array}$ \\
\hline $\begin{array}{l}\lambda_{7} \\
a_{7} \\
b_{7}\end{array}$ & & & & & & & & $\begin{array}{l}0.8137 \\
0.1072 \\
0.1566\end{array}$ & $\begin{array}{l}0.7326 \\
0.1617 \\
0.1409\end{array}$ \\
\hline $\begin{array}{l}\lambda_{8} \\
a_{8} \\
b_{8}\end{array}$ & & & & & & & $\begin{array}{c}0.9651 \\
-.0026 \\
0.0587\end{array}$ & & $\begin{array}{l}0.8439 \\
0.0737 \\
0.1341\end{array}$ \\
\hline $\begin{array}{l}\lambda_{9} \\
a_{9} \\
b_{y}\end{array}$ & & & & & & & & $\begin{array}{c}0.9710 \\
-.0041 \\
0.0449\end{array}$ & $\begin{array}{l}0.9236 \\
0.0184 \\
0.0886\end{array}$ \\
\hline 0 & & & & & & & & & $\begin{array}{c}0.9754 \\
-.0046 \\
0.0352\end{array}$ \\
\hline $\begin{array}{l}K_{1} \\
K_{2} \\
K_{3}\end{array}$ & $\begin{array}{l}0.5663 \\
0.7926 \\
0.0000 \\
0.4488\end{array}$ & $\begin{array}{l}0.6702 \\
0.8977 \\
0.0000 \\
0.6016\end{array}$ & $\begin{array}{l}0.6890 \\
1.0267 \\
0.0000 \\
0.7074\end{array}$ & $\begin{array}{l}0.7097 \\
1.0827 \\
0.0000 \\
0.7683\end{array}$ & $\begin{array}{l}0.7189 \\
1.1253 \\
0.0000 \\
0.8090\end{array}$ & $\begin{array}{l}0.7261 \\
1.1521 \\
0.0000 \\
0.8365\end{array}$ & $\begin{array}{l}0.7307 \\
1.1717 \\
0.0000 \\
0.8562\end{array}$ & $\begin{array}{l}0.7342 \\
1.1858 \\
0.0000 \\
0.8707\end{array}$ & $\begin{array}{l}0.7368 \\
1.1965 \\
0.0000 \\
0.8816\end{array}$ \\
\hline
\end{tabular}


Table A.5: Optimal Spacing for the location and scale parameters along with the coefficeints for the ABLUE of $t$-distribution.

\begin{tabular}{|c|c|c|c|c|c|c|c|c|c|}
\hline \multicolumn{10}{|c|}{ D.F. $=10$} \\
\hline$k$ & 2 & 3 & 4 & 5 & 6 & 7 & 8 & !) & 10 \\
\hline $\begin{array}{l}\lambda_{1} \\
a_{1} \\
b_{1}\end{array}$ & $\begin{array}{l}0.1702 \\
0.5000 \\
-.4994\end{array}$ & $\begin{array}{l}0.1122 \\
0.2002 \\
-.3860\end{array}$ & $\begin{array}{l}0.0699 \\
0.0852 \\
-.2217\end{array}$ & $\begin{array}{c}0.0499 \\
0.0458 \\
-.1511\end{array}$ & $\begin{array}{r}0.0363 \\
0.0255 \\
-.1038\end{array}$ & $\begin{array}{r}0.0277 \\
0.0153 \\
-.0758\end{array}$ & $\begin{array}{l}0.0217 \\
0.009 .1 \\
-.056 s\end{array}$ & $\begin{array}{l}0.0174 \\
0.0059 \\
-.0439\end{array}$ & $\begin{array}{r}0.0148 \\
0.0037 \\
-.0346\end{array}$ \\
\hline $\begin{array}{l}\lambda_{2} \\
a_{2} \\
b_{2}\end{array}$ & $\begin{array}{l}0.8298 \\
0.5000 \\
0.4994\end{array}$ & $\begin{array}{l}0.5000 \\
0.5995 \\
0.0000\end{array}$ & $\begin{array}{l}0.2849 \\
0.4148 \\
-.2459\end{array}$ & $\begin{array}{l}0.1974 \\
0.2393 \\
-.2543\end{array}$ & $\begin{array}{l}0.1406 \\
0.1388 \\
-.2048\end{array}$ & $\begin{array}{l}0.1062 \\
0.0879 \\
-.1616\end{array}$ & $\begin{array}{l}0.0824 \\
0.0580 \\
-.1270\end{array}$ & $\begin{array}{l}0.0658 \\
0.0399 \\
-.1014\end{array}$ & $\begin{array}{l}0.05360 \\
0.0282 \\
-.05^{2} 20\end{array}$ \\
\hline $\begin{array}{l}\lambda_{3} \\
a_{3} \\
b_{3}\end{array}$ & & $\begin{array}{l}0.8878 \\
0.2002 \\
0.3860\end{array}$ & $\begin{array}{l}0.7151 \\
0.4148 \\
0.2459\end{array}$ & $\begin{array}{l}0.5000 \\
0.4297 \\
0.0000\end{array}$ & $\begin{array}{l}0.3447 \\
0.3357 \\
-.1420\end{array}$ & $\begin{array}{l}0.2552 \\
0.2290 \\
-.1766\end{array}$ & $\begin{array}{c}0.1951 \\
0.1538 \\
-.1659\end{array}$ & $\begin{array}{l}0.15 .13 \\
0.107 . \\
-.1417\end{array}$ & $\begin{array}{l}0.1215 \\
0.107(64) \\
-.1233\end{array}$ \\
\hline $\begin{array}{l}\lambda_{4} \\
a_{4} \\
b_{4}\end{array}$ & & & $\begin{array}{l}0.9301 \\
0.0852 \\
0.2217\end{array}$ & $\begin{array}{l}0.8026 \\
0.2393 \\
0.2543\end{array}$ & $\begin{array}{l}0.6553 \\
0.3357 \\
0.1420\end{array}$ & $\begin{array}{l}0.5000 \\
0.3356 \\
0.0000\end{array}$ & $\begin{array}{l}0.3785 \\
0.2788 \\
-.0925\end{array}$ & $\begin{array}{l}0.29157 \\
0.2019 .8 \\
-.1266\end{array}$ & $\begin{array}{l}0.2367 \\
0.1537 \\
-.1327\end{array}$ \\
\hline $\begin{array}{l}\lambda_{5} \\
a_{5} \\
b_{5}\end{array}$ & & & & $\begin{array}{l}0.9501 \\
0.0458 \\
0.1511\end{array}$ & $\begin{array}{l}0.8594 \\
0.1388 \\
0.2048\end{array}$ & $\begin{array}{l}0.7448 \\
0.2290 \\
0.1766\end{array}$ & $\begin{array}{l}0.6218 \\
0.2788 \\
0.0925\end{array}$ & $\begin{array}{l}0.50(0) \\
0.2751 \\
0.00001\end{array}$ & $\begin{array}{r}0.4001 \\
0.2375 \\
-.065 .52\end{array}$ \\
\hline $\begin{array}{l}\lambda_{6} \\
a_{6} \\
b_{6}\end{array}$ & & & & & $\begin{array}{l}0.9637 \\
0.0255 \\
0.1038\end{array}$ & $\begin{array}{l}0.8938 \\
0.0879 \\
0.1616\end{array}$ & $\begin{array}{l}0.80 .19 \\
0.15 .38 \\
0.165 !\end{array}$ & $\begin{array}{l}0.701 .3 \\
0.200 .4 \\
0.12 \times 60\end{array}$ & $\begin{array}{l}0.59949 \\
0.2375 \\
0.06652\end{array}$ \\
\hline $\begin{array}{l}\lambda_{7} \\
a_{7} \\
b_{7}\end{array}$ & & & & & & $\begin{array}{l}0.972 .3 \\
0.0153 \\
0.0758\end{array}$ & $\begin{array}{l}0.9176 \\
0.05 \times 0 \\
0.1270\end{array}$ & $\begin{array}{l}0.8157 \\
0.1072 \\
0.1447\end{array}$ & $\begin{array}{l}0.76 .3 .33 \\
0.15 .37 \\
0.1327\end{array}$ \\
\hline $\begin{array}{l}\lambda_{8} \\
a_{8} \\
b_{8}\end{array}$ & & & & & & & $\begin{array}{l}0.978 .3 \\
0.0094 \\
0.0568\end{array}$ & $\begin{array}{l}0.9342 \\
0.03999 \\
0.1014\end{array}$ & $\begin{array}{l}0.8752 \\
0.0769 \\
0.1235\end{array}$ \\
\hline $\begin{array}{l}\lambda_{9} \\
a_{9} \\
b_{9}\end{array}$ & & & & & & & & $\begin{array}{l}0.9826 \\
0.0059 \\
0.0439\end{array}$ & $\begin{array}{l}0.94(; 1) \\
0.0282 \\
0.0820\end{array}$ \\
\hline $\begin{array}{l}\lambda_{10} \\
a_{10} \\
b_{10}\end{array}$ & & & & & & & & & $\begin{array}{l}0.9 \times 57 \\
0.00 .37 \\
0.0346\end{array}$ \\
\hline $\begin{array}{c}K_{1} \\
K_{2} \\
K_{3} \\
\Delta\end{array}$ & $\begin{array}{l}0.6222 \\
0.9455 \\
0.0000 \\
0.5883\end{array}$ & $\begin{array}{l}0.7473 \\
1.0613 \\
0.0000 \\
0.7924\end{array}$ & $\begin{array}{l}0.7687 \\
1.2342 \\
0.0000 \\
0.9487\end{array}$ & $\begin{array}{l}0.7962 \\
1.3035 \\
0.0000 \\
1.0379\end{array}$ & $\begin{array}{l}0.8071 \\
1.3627 \\
0.0000 \\
1.0998\end{array}$ & $\begin{array}{l}0.8166 \\
1.3984 \\
0.0000 \\
1.1418\end{array}$ & $\begin{array}{l}0.8222 \\
1.4259 \\
0.0000 \\
1.1724\end{array}$ & $\begin{array}{l}0.82677 \\
1.4455 \\
0.0000 \\
1.1949\end{array}$ & $\begin{array}{l}0.8235 \\
1.4606 \\
0.0000 \\
1.2121\end{array}$ \\
\hline
\end{tabular}


Table A.6: Optimal Spacing for the location and scale parameters along with the coefficeints for the ABLUE of $t$-distribution.

\begin{tabular}{|c|c|c|c|c|c|c|c|c|c|}
\hline \multicolumn{10}{|c|}{ D.F. $=20$} \\
\hline$k$ & 2 & 3 & 4 & 5 & 6 & 7 & 8 & 9 & 10 \\
\hline $\begin{array}{l}\lambda_{1} \\
a_{1} \\
b_{1}\end{array}$ & $\begin{array}{l}0.1525 \\
0.5000 \\
-.4750\end{array}$ & $\begin{array}{c}0.0981 \\
0.2122 \\
-.3739\end{array}$ & $\begin{array}{c}0.0575 \\
0.0967 \\
-.2119\end{array}$ & $\begin{array}{c}0.0398 \\
0.0566 \\
-.1460\end{array}$ & $\begin{array}{l}0.0277 \\
0.0343 \\
-.1000\end{array}$ & $\begin{array}{r}0.0206 \\
0.0227 \\
-.0734\end{array}$ & $\begin{array}{r}0.0156 \\
0.0155 \\
-.0550\end{array}$ & $\begin{array}{c}0.0122 \\
0.0110 \\
-.0426\end{array}$ & $\begin{array}{r}0.0097 \\
0.0080 \\
-.0336\end{array}$ \\
\hline $\begin{array}{l}\lambda_{2} \\
a_{2} \\
b_{2}\end{array}$ & $\begin{array}{l}0.8475 \\
0.5000 \\
0.4750\end{array}$ & $\begin{array}{l}0.5000 \\
0.5757 \\
0.0000\end{array}$ & $\begin{array}{c}0.2668 \\
0.4033 \\
-.2380\end{array}$ & $\begin{array}{l}0.1795 \\
0.2366 \\
-.2453\end{array}$ & $\begin{array}{l}0.1226 \\
0.1402 \\
-.1958\end{array}$ & $\begin{array}{c}0.0900 \\
0.0921 \\
-.1544\end{array}$ & $\begin{array}{c}0.0676 \\
0.0629 \\
-.1210\end{array}$ & $\begin{array}{r}0.0526 \\
0.0450 \\
-.0966\end{array}$ & $\begin{array}{r}0.0417 \\
0.0331 \\
-.0780\end{array}$ \\
\hline $\begin{array}{l}\lambda_{3} \\
a_{3} \\
b_{3}\end{array}$ & & $\begin{array}{l}0.9019 \\
0.2122 \\
0.3739\end{array}$ & $\begin{array}{l}0.7332 \\
0.4033 \\
0.2380\end{array}$ & $\begin{array}{l}0.5000 \\
0.4135 \\
0.0000\end{array}$ & $\begin{array}{l}0.3299 \\
0.3254 \\
-.1392\end{array}$ & $\begin{array}{c}0.2371 \\
0.2230 \\
-.1717\end{array}$ & & $\begin{array}{l}0.1353 \\
0.1073 \\
-.1386\end{array}$ & $\begin{array}{l}0.1066 \\
0.0786 \\
-.1177\end{array}$ \\
\hline $\begin{array}{l}\lambda_{4} \\
a_{4} \\
b_{4}\end{array}$ & & & $\begin{array}{l}0.9425 \\
0.0967 \\
0.2119\end{array}$ & $\begin{array}{l}0.8205 \\
0.2366 \\
0.2453\end{array}$ & $\begin{array}{l}0.6701 \\
0.3254 \\
0.1392\end{array}$ & $\begin{array}{l}0.5000 \\
0.3244 \\
0.0000\end{array}$ & $\begin{array}{l}0.3664 \\
0.2704 \\
-.0915\end{array}$ & & $\begin{array}{l}0.2172 \\
0.1496 \\
-.1288\end{array}$ \\
\hline $\begin{array}{l}\lambda_{5} \\
a_{5} \\
b_{5}\end{array}$ & & & & $\begin{array}{l}0.9602 \\
0.0566 \\
0.1460\end{array}$ & $\begin{array}{l}0.8774 \\
0.1402 \\
0.1958\end{array}$ & $\begin{array}{l}0.7629 \\
0.2230 \\
0.1717\end{array}$ & $\begin{array}{l}0.6336 \\
0.2704 \\
0.0915\end{array}$ & $\begin{array}{l}0.5000 \\
0.2670 \\
0.0000\end{array}$ & $\begin{array}{l}0.3898 \\
0.2307 \\
-.0650\end{array}$ \\
\hline $\begin{array}{l}\lambda_{i} \\
a_{i ;} \\
b_{i}\end{array}$ & & & & & $\begin{array}{l}0.9723 \\
0.0343 \\
0.1000\end{array}$ & $\begin{array}{l}0.9100 \\
0.0921 \\
0.1544\end{array}$ & $\begin{array}{l}0.8244 \\
0.1512 \\
0.1598\end{array}$ & $\begin{array}{l}0.7214 \\
0.2032 \\
0.1261\end{array}$ & $\begin{array}{l}0.6102 \\
0.2307 \\
0.0650\end{array}$ \\
\hline $\begin{array}{l}\lambda_{7} \\
a_{7} \\
b_{7}\end{array}$ & & & & & & $\begin{array}{l}0.9794 \\
0.0227 \\
0.0734\end{array}$ & $\begin{array}{l}0.9324 \\
0.0629 \\
0.1210\end{array}$ & $\begin{array}{l}0.8647 \\
0.1073 \\
0.1386\end{array}$ & $\begin{array}{l}0.7828 \\
0.1496 \\
0.1288\end{array}$ \\
\hline $\begin{array}{l}\lambda_{8} \\
a_{8} \\
b_{8}\end{array}$ & & & & & & & $\begin{array}{l}0.9844 \\
0.0155 \\
0.0550\end{array}$ & $\begin{array}{l}0.9474 \\
0.0450 \\
0.0966\end{array}$ & $\begin{array}{l}0.8934 \\
C .0786 \\
0.1177\end{array}$ \\
\hline $\begin{array}{l}\lambda_{9} \\
a_{9} \\
b_{9}\end{array}$ & & & & & & & & $\begin{array}{l}0.9878 \\
0.0110 \\
0.0426\end{array}$ & $\begin{array}{l}0.9583 \\
0.0331 \\
0.0780\end{array}$ \\
\hline 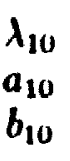 & & & & & & & & & $\begin{array}{l}0.9903 \\
0.0080 \\
0.0336\end{array}$ \\
\hline $\begin{array}{l}\boldsymbol{K}_{1} \\
\boldsymbol{h}_{2} \\
\boldsymbol{K}_{3} \\
\Delta\end{array}$ & $\begin{array}{l}0.6559 \\
1.0459 \\
0.0000 \\
0.6860\end{array}$ & $\begin{array}{l}0.7958 \\
1.1659 \\
0.0000 \\
0.9278\end{array}$ & $\begin{array}{l}0.8197 \\
1.3735 \\
0.0000 \\
1.1258\end{array}$ & $\begin{array}{l}0.8531 \\
1.4513 \\
0.0000 \\
1.2381\end{array}$ & $\begin{array}{l}0.8655 \\
1.5234 \\
0.0000 \\
1.3186\end{array}$ & $\begin{array}{l}0.8772 \\
1.5655 \\
0.0000 \\
1.3732\end{array}$ & $\begin{array}{l}0.8838 \\
1.5992 \\
0.0000 \\
1.4134\end{array}$ & $\begin{array}{l}0.8893 \\
1.6229 \\
0.0000 \\
1.4432\end{array}$ & $\begin{array}{l}0.8931 \\
1.6416 \\
0.0000 \\
1.4661\end{array}$ \\
\hline
\end{tabular}


Table A.7: Optimal Spacing for the location and scale parameters along with the coefficeints for the ABLUE of $t$-distribution.

\begin{tabular}{|c|c|c|c|c|c|c|c|c|c|}
\hline \multicolumn{10}{|c|}{ D.F. $=500$} \\
\hline$k$ & 2 & 3 & 4 & 5 & 6 & 7 & 8 & 9 & 10 \\
\hline $\begin{array}{l}\lambda_{1} \\
a_{1} \\
b_{1}\end{array}$ & $\begin{array}{c}0.1342 \\
0.5000 \\
-.4512\end{array}$ & $\begin{array}{l}\mathbf{0 . 0 8 3 9} \\
\mathbf{0 . 2 2 3 9} \\
-. .3619\end{array}$ & $\begin{array}{l}0.0454 \\
0.1079 \\
-.2017\end{array}$ & $\begin{array}{c}0.0303 \\
0.0671 \\
-.1408\end{array}$ & $\begin{array}{l}0.0200 \\
0.0428 \\
-.0959\end{array}$ & $\begin{array}{c}0.0143 \\
0.0298 \\
-.0707\end{array}$ & $\begin{array}{l}0.0104 \\
0.0213 \\
-.0528\end{array}$ & $\begin{array}{l}0.0079 \\
0.0159 \\
-.0410\end{array}$ & $\begin{array}{l}0.0061 \\
0.0121 \\
-.0323\end{array}$ \\
\hline $\begin{array}{l}\lambda_{2} \\
a_{2} \\
b_{2}\end{array}$ & $\begin{array}{l}0.8658 \\
0.5000 \\
0.4512\end{array}$ & $\begin{array}{l}0.5000 \\
0.5521 \\
0.0000\end{array}$ & $\begin{array}{l}0.2462 \\
0.3921 \\
-.2303\end{array}$ & $\begin{array}{c}0.1602 \\
0.2344 \\
-.2365\end{array}$ & $\begin{array}{l}0.1038 \\
0.1419 \\
-.1867\end{array}$ & $\begin{array}{l}0.0735 \\
0.0964 \\
-.1472\end{array}$ & $\begin{array}{c}0.0530 \\
0.0678 \\
-.1149\end{array}$ & $\begin{array}{l}0.03999 \\
0.0501 \\
-.0917\end{array}$ & $\begin{array}{r}0.0306 \\
0.0379 \\
-.0734\end{array}$ \\
\hline $\begin{array}{l}\lambda_{3} \\
a_{3} \\
b_{3}\end{array}$ & & $\begin{array}{l}0.9161 \\
0.2239 \\
0.3619\end{array}$ & $\begin{array}{l}0.7538 \\
0.3921 \\
0.2303\end{array}$ & $\begin{array}{l}0.5000 \\
0.3970 \\
0.0000\end{array}$ & $\begin{array}{c}0.3122 \\
\mathbf{0 . 3 1 5 3} \\
-.1368\end{array}$ & $\begin{array}{l}0.2168 \\
0.2173 \\
-.1670\end{array}$ & $\begin{array}{l}0.1543 \\
0.1488 \\
-.1538\end{array}$ & $\begin{array}{l}0.1151 \\
0.1077 \\
-.1327\end{array}$ & $\begin{array}{l}0.0877 \\
0.080 .4 \\
-.1121\end{array}$ \\
\hline $\begin{array}{l}\lambda_{4} \\
a_{4} \\
b_{4}\end{array}$ & & & $\begin{array}{l}0.9546 \\
0.1079 \\
0.2017\end{array}$ & $\begin{array}{l}0.8398 \\
0.2344 \\
0.2365\end{array}$ & $\begin{array}{l}0.6878 \\
0.3153 \\
0.1368\end{array}$ & $\begin{array}{l}0.5000 \\
0.3129 \\
0.0000\end{array}$ & $\begin{array}{l}0.2516 \\
0.2621 \\
-.0910\end{array}$ & $\begin{array}{l}0.2588 \\
0.1970 \\
-.1239\end{array}$ & $\begin{array}{r}0.1953 \\
0.1456 \\
-.1252\end{array}$ \\
\hline $\begin{array}{l}\lambda_{5} \\
a_{5} \\
b_{5}\end{array}$ & & & & $\begin{array}{l}0.9697 \\
0.0671 \\
0.1408\end{array}$ & $\begin{array}{l}0.8962 \\
0.1419 \\
0.1867\end{array}$ & $\begin{array}{l}0.7832 \\
0.2173 \\
0.1670\end{array}$ & $\begin{array}{l}0.6481 \\
0.2621 \\
0.0910\end{array}$ & $\begin{array}{l}0.5000 \\
0.2586 \\
0.0000\end{array}$ & $\begin{array}{r}0.3773 \\
0.2240 \\
-.06 .31\end{array}$ \\
\hline $\begin{array}{l}\lambda_{6} \\
a_{6} \\
b_{6}\end{array}$ & & & & & $\begin{array}{l}0.9800 \\
0.0428 \\
0.0959\end{array}$ & $\begin{array}{l}0.9265 \\
0.0964 \\
0.1472\end{array}$ & $\begin{array}{l}0.8457 \\
0.1488 \\
0.1538\end{array}$ & $\begin{array}{l}0.7412 \\
0.1970 \\
0.1239\end{array}$ & $\begin{array}{l}0.6227 \\
0.2240 \\
0.0651\end{array}$ \\
\hline $\begin{array}{l}\lambda_{7} \\
a_{7} \\
b_{7}\end{array}$ & & & & & & $\begin{array}{l}0.9857 \\
0.0298 \\
0.0707\end{array}$ & $\begin{array}{l}0.9470 \\
0.0678 \\
0.1149\end{array}$ & $\begin{array}{l}0.8819 \\
0.1077 \\
0.1327\end{array}$ & $\begin{array}{l}0.8047 \\
0.1456 \\
0.1252\end{array}$ \\
\hline $\begin{array}{l}\lambda_{8} \\
a_{8} \\
b_{8}\end{array}$ & & & & & & & $\begin{array}{l}0.9896 \\
0.0213 \\
0.0528\end{array}$ & $\begin{array}{l}0.9601 \\
0.0501 \\
0.0917\end{array}$ & $\begin{array}{l}0.9123 \\
0.0801 \\
0.1121\end{array}$ \\
\hline $\begin{array}{l}\lambda_{9} \\
a_{9} \\
b_{9}\end{array}$ & & & & & & & & $\begin{array}{l}0.9921 \\
0.0159 \\
0.0410\end{array}$ & $\begin{array}{l}0.9694 \\
0.0379 \\
0.0739\end{array}$ \\
\hline $\begin{array}{l}\lambda_{10} \\
a_{10} \\
b_{10}\end{array}$ & & & & & & & & & $\begin{array}{l}0.9939 \\
0.0121 \\
0.0323\end{array}$ \\
\hline $\begin{array}{c}K_{1} \\
K_{2} \\
K_{3} \\
\Delta\end{array}$ & $\begin{array}{l}0.6935 \\
1.1639 \\
0.0000 \\
0.8072\end{array}$ & $\begin{array}{l}0.8511 \\
1.2891 \\
0.0000 \\
1.0972\end{array}$ & $\begin{array}{l}0.8789 \\
1.5407 \\
0.0000 \\
1.3541\end{array}$ & $\begin{array}{l}0.9204 \\
1.6282 \\
0.0000 \\
1.4985\end{array}$ & $\begin{array}{l}0.9351 \\
1.7179 \\
0.0000 \\
1.6064\end{array}$ & $\begin{array}{l}0.9500 \\
1.7679 \\
0.0000 \\
1.6796\end{array}$ & $\begin{array}{l}0.9582 \\
1.8103 \\
0.0000 \\
1.7346\end{array}$ & $\begin{array}{l}0.965 .33 \\
1.8393 \\
0.0000 \\
1.7754\end{array}$ & $\begin{array}{l}0.9701 \\
1.8620 \\
0.0000 \\
1.8072\end{array}$ \\
\hline
\end{tabular}


Appendix A

t-Dist. (Regression Parameters, p=1 ) 
Table A.1: Optimal Spacing for the regression and scale paraneters along with the coefficeints for the ABLUE of $t$-distribution with $p=1$.

\begin{tabular}{|c|c|c|c|c|c|c|c|c|}
\hline \multicolumn{9}{|c|}{$D . F .=1$} \\
\hline$k$ & 3 & 4 & 5 & 6 & 7 & 8 & 9 & 10 \\
\hline $\begin{array}{l}\lambda_{1} \\
a_{1}\end{array}$ & $\begin{array}{l}0.2500 \\
0.0000\end{array}$ & $\begin{array}{l}0.1837 \\
-.0769\end{array}$ & $\begin{array}{r}0.1535 \\
-.0730\end{array}$ & $\begin{array}{l}0.1321 \\
-.0593\end{array}$ & $\begin{array}{l}0.1147 \\
-.0446\end{array}$ & $\begin{array}{r}0.1013 \\
-.0333\end{array}$ & $\begin{array}{l}0.0911 \\
-.025 .54\end{array}$ & $\begin{array}{l}0.08: 27 \\
-.01 ! 97\end{array}$ \\
\hline $\begin{array}{l}\lambda_{2} \\
a_{2}\end{array}$ & $\begin{array}{l}0.5000 \\
1.0000\end{array}$ & $\begin{array}{l}0.4099 \\
0.5769\end{array}$ & $\begin{array}{l}0.3465 \\
0.2664\end{array}$ & $\begin{array}{l}0.2941 \\
0.0892\end{array}$ & $\begin{array}{l}0.2500 \\
0.0000\end{array}$ & $\begin{array}{l}0.2170 \\
-.0350\end{array}$ & $\begin{array}{l}0.1426 \\
-.0153\end{array}$ & $\begin{array}{l}0.1732 \\
-.045 .1\end{array}$ \\
\hline $\begin{array}{l}\lambda_{3} \\
a_{3}\end{array}$ & $\begin{array}{l}0.7500 \\
0.0000\end{array}$ & $\begin{array}{l}0.5901 \\
0.5769\end{array}$ & $\begin{array}{l}0.5000 \\
0.6132\end{array}$ & $\begin{array}{l}0.4364 \\
0.4701\end{array}$ & $\begin{array}{l}0.3853 \\
0.3147\end{array}$ & $\begin{array}{l}0.3431 \\
0.1860\end{array}$ & $\begin{array}{l}0.3071 \\
0.0911\end{array}$ & $\begin{array}{l}0.27(i 5) \\
0.03151\end{array}$ \\
\hline $\begin{array}{l}\lambda_{4} \\
a_{4}\end{array}$ & & $\begin{array}{r}0.8163 \\
-.0769\end{array}$ & $\begin{array}{l}0.6535 \\
0.2664\end{array}$ & $\begin{array}{l}0.5636 \\
0.4701\end{array}$ & $\begin{array}{l}0.5000 \\
0.4599\end{array}$ & $\begin{array}{l}0.4501 \\
0.3823\end{array}$ & $\begin{array}{l}0.4069 \\
0.293 .1\end{array}$ & $\begin{array}{l}0.3736 \\
0.2117\end{array}$ \\
\hline $\begin{array}{l}\lambda_{5} \\
a_{5}\end{array}$ & & & $\begin{array}{r}0.8465 \\
-.0730\end{array}$ & $\begin{array}{l}0.7059 \\
0.0892\end{array}$ & $\begin{array}{l}0.6147 \\
0.3147\end{array}$ & $\begin{array}{l}0.5499 \\
0.3823\end{array}$ & $\begin{array}{l}0.50600 \\
0.3650\end{array}$ & $\begin{array}{l}0.4591 \\
0.31 \times 0\end{array}$ \\
\hline $\begin{array}{l}\lambda_{6} \\
a_{6}\end{array}$ & & & & $\begin{array}{r}0.8679 \\
-.0593\end{array}$ & $\begin{array}{l}0.7500 \\
0.0000\end{array}$ & $\begin{array}{l}0.65699 \\
0.1860\end{array}$ & $\begin{array}{l}0.5911 \\
0.29 ! 14\end{array}$ & $\begin{array}{l}0.5 .10 ! \\
0.31: 0\end{array}$ \\
\hline $\begin{array}{l}\lambda_{i} \\
a_{i}\end{array}$ & & & & & $\begin{array}{c}0.88533 \\
-.0446\end{array}$ & $\begin{array}{r}0.7830 \\
-.0350\end{array}$ & $\begin{array}{l}0.6926 \\
0.0918\end{array}$ & $\begin{array}{l}0.626 .1 \\
0.2117\end{array}$ \\
\hline $\begin{array}{l}\lambda_{8} \\
a_{8}\end{array}$ & & & & & & $\begin{array}{c}0.8987 \\
-.0333\end{array}$ & $\begin{array}{r}0.8074 \\
-.0453\end{array}$ & $\begin{array}{l}0.72351 \\
0.0351\end{array}$ \\
\hline $\begin{array}{l}\lambda_{9} \\
a_{9}\end{array}$ & & & & & & & $\begin{array}{r}0.90 \times 9 \\
-.025 .4\end{array}$ & $\begin{array}{l}0.826 \% \\
-.0454\end{array}$ \\
\hline $\begin{array}{l}\lambda_{10} \\
a_{10}\end{array}$ & & & & & & & & $\begin{array}{l}0.9173 \\
-.0197\end{array}$ \\
\hline $\begin{array}{c}K_{1} \\
K_{2} \\
K_{3} \\
\Delta\end{array}$ & $\begin{array}{l}0.4053 \\
0.4053 \\
0.0000 \\
0.0666\end{array}$ & $\begin{array}{l}0.4469 \\
0.4244 \\
0.0000 \\
0.0848\end{array}$ & $\begin{array}{l}0.4631 \\
0.4457 \\
0.0000 \\
0.0956\end{array}$ & $\begin{array}{l}0.4712 \\
0.4619 \\
0.0000 \\
0.1025\end{array}$ & $\begin{array}{l}0.4774 \\
0.4713 \\
0.0000 \\
0.1074\end{array}$ & $\begin{array}{l}0.4822 \\
0.4770 \\
0.0000 \\
0.1109\end{array}$ & $\begin{array}{l}0.485,5 \\
0.4813 \\
0.0000 \\
0.1135\end{array}$ & $\begin{array}{l}0.4880 \\
0.4846 \\
0.0000 \\
0.1154\end{array}$ \\
\hline
\end{tabular}


Table A.2: Optimal Spacing for the regression and scale parameters along with the coefficeints for the ABLUE of t-distribution with $p=1$.

\begin{tabular}{|c|c|c|c|c|c|c|c|c|}
\hline \multicolumn{9}{|c|}{ D.F. $=2$} \\
\hline$k$ & 3 & 4 & 5 & 6 & 7 & 8 & 9 & 10 \\
\hline $\begin{array}{l}\lambda_{1} \\
a_{1}\end{array}$ & $\begin{array}{l}0.2152 \\
0.1330\end{array}$ & $\begin{array}{l}0.1578 \\
0.0087\end{array}$ & $\begin{array}{l}0.1204 \\
-.0273\end{array}$ & $\begin{array}{r}0.0955 \\
-.0339\end{array}$ & $\begin{array}{l}0.0781 \\
-.0317\end{array}$ & $\begin{array}{l}0.0654 \\
-.0272\end{array}$ & $\begin{array}{l}0.0558 \\
-.0227\end{array}$ & $\begin{array}{r}0.0483 \\
-.0188\end{array}$ \\
\hline $\begin{array}{l}\lambda_{2} \\
a_{2}\end{array}$ & $\begin{array}{l}0.5000 \\
0.7341\end{array}$ & $\begin{array}{l}0.3837 \\
0.4913\end{array}$ & $\begin{array}{l}0.3047 \\
0.2781\end{array}$ & $\begin{array}{l}0.2479 \\
0.1437\end{array}$ & $\begin{array}{l}0.2057 \\
0.0663\end{array}$ & $\begin{array}{l}0.1736 \\
0.0237\end{array}$ & $\begin{array}{l}0.1487 \\
0.0009\end{array}$ & $\begin{array}{l}0.1290 \\
-.0106\end{array}$ \\
\hline $\begin{array}{l}\lambda_{3} \\
a_{3}\end{array}$ & $\begin{array}{l}0.7848 \\
0.1330\end{array}$ & $\begin{array}{l}0.6163 \\
0.4913\end{array}$ & $\begin{array}{l}0.5000 \\
0.4983\end{array}$ & $\begin{array}{l}0.4156 \\
0.3902\end{array}$ & $\begin{array}{l}0.3515 \\
0.2774\end{array}$ & $\begin{array}{l}0.3015 \\
0.1870\end{array}$ & $\begin{array}{l}0.2615 \\
0.1208\end{array}$ & $\begin{array}{l}0.2290 \\
0.0746\end{array}$ \\
\hline $\begin{array}{l}\lambda_{4} \\
a_{4}\end{array}$ & & $\begin{array}{l}0.8422 \\
0.0087\end{array}$ & $\begin{array}{l}0.6953 \\
0.2781\end{array}$ & $\begin{array}{l}0.5844 \\
0.3902\end{array}$ & $\begin{array}{l}0.5000 \\
0.3758\end{array}$ & $\begin{array}{l}0.4337 \\
0.3166\end{array}$ & $\begin{array}{l}0.3803 \\
0.2501\end{array}$ & $\begin{array}{l}0.3364 \\
0.1901\end{array}$ \\
\hline $\begin{array}{l}\lambda_{5} \\
a_{5}\end{array}$ & & & $\begin{array}{r}0.8796 \\
-.0273\end{array}$ & $\begin{array}{l}0.7521 \\
0.1437\end{array}$ & $\begin{array}{l}0.6485 \\
0.2774\end{array}$ & $\begin{array}{l}0.5663 \\
0.3166\end{array}$ & $\begin{array}{l}0.5000 \\
0.3018\end{array}$ & $\begin{array}{l}0.4454 \\
0.2647\end{array}$ \\
\hline $\begin{array}{l}\lambda_{6} \\
a_{6}\end{array}$ & & & & $\begin{array}{c}0.9045 \\
-.0339\end{array}$ & $\begin{array}{l}0.7943 \\
0.0663\end{array}$ & $\begin{array}{l}0.6985 \\
0.1870\end{array}$ & $\begin{array}{l}0.6197 \\
0.2501\end{array}$ & $\begin{array}{l}0.5546 \\
0.2647\end{array}$ \\
\hline $\begin{array}{l}\lambda_{i} \\
a_{i}\end{array}$ & & & & & $\begin{array}{r}0.9219 \\
-.0317\end{array}$ & $\begin{array}{l}0.8264 \\
0.0237\end{array}$ & $\begin{array}{l}0.7385 \\
0.1208\end{array}$ & $\begin{array}{l}0.6636 \\
0.1901\end{array}$ \\
\hline $\begin{array}{l}\lambda_{8} \\
a_{8}\end{array}$ & & & & & & $\begin{array}{r}0.9346 \\
-.0272\end{array}$ & $\begin{array}{l}0.8513 \\
0.0009\end{array}$ & $\begin{array}{l}0.7710 \\
0.0746\end{array}$ \\
\hline $\begin{array}{l}\lambda_{9} \\
a_{9}\end{array}$ & & & & & & & $\begin{array}{c}0.9442 \\
-.0227\end{array}$ & $\begin{array}{r}0.8710 \\
-.0106\end{array}$ \\
\hline $\begin{array}{l}\lambda_{10} \\
a_{10}\end{array}$ & & & & & & & & $\begin{array}{r}0.9517 \\
-.0188\end{array}$ \\
\hline $\begin{array}{c}\boldsymbol{K}_{1} \\
\boldsymbol{K}_{2} \\
\boldsymbol{K}_{3} \\
\boldsymbol{\Delta}\end{array}$ & $\begin{array}{l}0.5318 \\
0.6040 \\
0.0000 \\
0.1708\end{array}$ & $\begin{array}{l}0.5516 \\
0.6733 \\
0.0000 \\
0.2049\end{array}$ & $\begin{array}{l}0.5648 \\
0.7103 \\
0.0000 \\
0.2266\end{array}$ & $\begin{array}{l}0.5735 \\
0.7330 \\
0.0000 \\
0.2411\end{array}$ & $\begin{array}{l}0.5793 \\
0.7481 \\
0.0000 \\
0.2510\end{array}$ & $\begin{array}{l}0.5834 \\
0.7586 \\
0.0000 \\
0.2582\end{array}$ & $\begin{array}{l}0.5864 \\
0.7662 \\
0.0000 \\
0.2635\end{array}$ & $\begin{array}{l}0.5887 \\
0.7719 \\
0.0000 \\
0.2675\end{array}$ \\
\hline
\end{tabular}


Table A.3: Optimal Spacing for the regression and scale parameters along with the coefficeints for the ABLUE of $t$-distribution with $p=1$.

$$
\text { D.F. }=\mathbf{3}
$$

\begin{tabular}{|c|c|c|c|c|c|c|c|c|}
\hline$k$ & 3 & 4 & 5 & 6 & 7 & 8 & 9 & 10 \\
\hline $\begin{array}{l}\lambda_{1} \\
a_{1}\end{array}$ & $\begin{array}{l}0.1862 \\
0.1694\end{array}$ & $\begin{array}{l}0.1354 \\
0.0505\end{array}$ & $\begin{array}{l}0.1027 \\
0.0061\end{array}$ & $\begin{array}{r}0.0805 \\
-.0103\end{array}$ & $\begin{array}{l}0.0648 \\
-.0155\end{array}$ & $\begin{array}{r}0.0533 \\
-.0163\end{array}$ & & $\begin{array}{c}0.0381 \\
-.01: 38\end{array}$ \\
\hline $\begin{array}{l}\lambda_{2} \\
a_{2}\end{array}$ & $\begin{array}{l}0.5000 \\
0.6612\end{array}$ & $\begin{array}{l}0.3661 \\
0.4495\end{array}$ & & $\begin{array}{l}0.2234 \\
0.1550\end{array}$ & $\begin{array}{l}1819 \\
0873\end{array}$ & $\begin{array}{l}0.1512 \\
0.0473\end{array}$ & $\begin{array}{l}0.1277 \\
0.02 \pm 37\end{array}$ & $\begin{array}{l}0.10 ! 9.9 \\
0.000 ! 96\end{array}$ \\
\hline $\begin{array}{l}\lambda_{3} \\
a_{3}\end{array}$ & $\begin{array}{l}0.8138 \\
0.1694\end{array}$ & $\begin{array}{l}0.6339 \\
0.4495\end{array}$ & $\begin{array}{l}0.5000 \\
0.4503\end{array}$ & $\begin{array}{l}0.4035 \\
0.3553\end{array}$ & & $\begin{array}{l}0.2787 \\
0.1 \times 0.4\end{array}$ & $\begin{array}{l}0.2372 \\
0.12 .12\end{array}$ & $\begin{array}{l}0.20 .41 \\
0.0 \times 1.1\end{array}$ \\
\hline $\begin{array}{l}\lambda_{4} \\
a_{4}\end{array}$ & & $\begin{array}{l}0.8646 \\
0.0505\end{array}$ & & & & & & $\begin{array}{l}0.31(i 0) \\
0.17 \times 10\end{array}$ \\
\hline $\begin{array}{l}\lambda_{3} \\
a_{5}\end{array}$ & & & $\begin{array}{l}0.8973 \\
0.0061\end{array}$ & $\begin{array}{l}0.7766 \\
0.1550\end{array}$ & & & & $\begin{array}{l}0.4376 \\
0.2418\end{array}$ \\
\hline $\begin{array}{l}\lambda_{6} \\
a_{6}\end{array}$ & & & & $\begin{array}{r}0.9195 \\
-.0103\end{array}$ & & & & $\begin{array}{l}0.51624 \\
0.2418\end{array}$ \\
\hline $\begin{array}{l}\lambda_{i} \\
a_{i}\end{array}$ & & & & & $\begin{array}{r}0.9352 \\
-.0155\end{array}$ & $\begin{array}{l}0.8488 \\
0.0473\end{array}$ & $\begin{array}{l}0.762 x \\
0.1242\end{array}$ & $\begin{array}{l}0.68 .10 \\
0.1780\end{array}$ \\
\hline $\begin{array}{l}\lambda_{8} \\
a_{8}\end{array}$ & & & & & & $\begin{array}{l}0.9467 \\
-.0163\end{array}$ & $\begin{array}{l}0.8723 \\
0.02: 37\end{array}$ & $\begin{array}{l}0.7954 \\
0.0844\end{array}$ \\
\hline $\begin{array}{l}\lambda_{9} \\
a_{9}\end{array}$ & & & & & & & $\begin{array}{r}0.955 .3 \\
-.015 .4\end{array}$ & $\begin{array}{l}0.89106 \\
0.0097\end{array}$ \\
\hline $\begin{array}{l}\lambda_{10} \\
a_{10}\end{array}$ & & & & & & & & $\begin{array}{l}0.96(1) \\
-.01:\end{array}$ \\
\hline $\begin{array}{c}K_{1} \\
K_{2} \\
K_{3} \\
\Delta\end{array}$ & $\begin{array}{l}0.6030 \\
0.7293 \\
0.0000 \\
0.2651\end{array}$ & $\begin{array}{l}0.6224 \\
0.8211 \\
0.0000 \\
0.3180\end{array}$ & $\begin{array}{l}0.6340 \\
0.8734 \\
0.0000 \\
0.3511\end{array}$ & $\begin{array}{l}0.6417 \\
0.9058 \\
0.0000 \\
0.3730\end{array}$ & $\begin{array}{l}0.6470 \\
0.9272 \\
0.0000 \\
0.3881\end{array}$ & $\begin{array}{l}0.6507 \\
0.9420 \\
0.0000 \\
0.3989\end{array}$ & $\begin{array}{l}0.65336 \\
0.95527 \\
0.0000 \\
0.4069\end{array}$ & $\begin{array}{l}0.65557 \\
0.9607 \\
0.0600 \\
0.41: 36)\end{array}$ \\
\hline
\end{tabular}


Table A.4: Optimal Spacing for the regression and scale parameters along with the cuefficeints for the ABLUE of $t$-distribution with $p=1$.

\begin{tabular}{|c|c|c|c|c|c|c|c|c|}
\hline \multirow[b]{2}{*}{$k$} & \multicolumn{8}{|c|}{$D . F .=5$} \\
\hline & 2 & 4 & 5 & 6 & 7 & 8 & 9 & 10 \\
\hline $\begin{array}{l}\lambda_{1} \\
a_{1}\end{array}$ & $\begin{array}{l}0.1553 \\
0.1966\end{array}$ & $\begin{array}{l}0.1084 \\
0.0824\end{array}$ & $\begin{array}{l}0.0799 \\
0.0355\end{array}$ & $\begin{array}{l}0.0613 \\
0.0143\end{array}$ & $\begin{array}{l}0.0485 \\
0.0041\end{array}$ & $\begin{array}{r}0.0393 \\
-.0008\end{array}$ & $\begin{array}{r}0.0325 \\
-.0032\end{array}$ & $\begin{array}{r}0.0273 \\
-.0043\end{array}$ \\
\hline $\begin{array}{l}\lambda_{2} \\
a_{2}\end{array}$ & $\begin{array}{l}0.5000 \\
0.6068\end{array}$ & $\begin{array}{l}0.3438 \\
0.4176\end{array}$ & $\begin{array}{l}0.2523 \\
0.2570\end{array}$ & $\begin{array}{l}0.1933 \\
0.1574\end{array}$ & $\begin{array}{l}0.1529 \\
0.0981\end{array}$ & & $\begin{array}{l}0.102 \pi \\
0.0398\end{array}$ & $\begin{array}{l}0.0864 \\
0.0256\end{array}$ \\
\hline $\begin{array}{l}\lambda_{3} \\
a_{3}\end{array}$ & $\begin{array}{l}0.8447 \\
0.1966\end{array}$ & $\begin{array}{l}0.6562 \\
0.4176\end{array}$ & & & & & & \\
\hline $\begin{array}{l}\lambda_{4} \\
a_{4}\end{array}$ & & $\begin{array}{l}0.8916 \\
0.0824\end{array}$ & $\begin{array}{l}0.7477 \\
0.2570\end{array}$ & & & & & \\
\hline $\begin{array}{l}\lambda_{\mathrm{s}} \\
a_{\mathrm{s}}\end{array}$ & & & $\begin{array}{l}0.9201 \\
0.0355\end{array}$ & $\begin{array}{l}0.8067 \\
0.1574\end{array}$ & $\begin{array}{l}0.6918 \\
0.2402\end{array}$ & & $\begin{array}{l}0.5000 \\
0.2544\end{array}$ & \\
\hline $\begin{array}{l}\lambda_{6} \\
a_{6}\end{array}$ & & & & $\begin{array}{l}0.9387 \\
0.0143\end{array}$ & & & & \\
\hline $\begin{array}{l}\lambda_{i} \\
a_{i}\end{array}$ & & & & & $\begin{array}{l}0.9515 \\
0.0041\end{array}$ & $\begin{array}{l}0.8759 \\
0.0621\end{array}$ & $\begin{array}{l}0.7922 \\
0.1229\end{array}$ & $\begin{array}{l}0.7092 \\
0.1665\end{array}$ \\
\hline $\begin{array}{l}\lambda_{*} \\
a_{8}\end{array}$ & & & & & & $\begin{array}{l}0.9607 \\
-.0008\end{array}$ & $\begin{array}{l}0.8973 \\
0.0398\end{array}$ & $\begin{array}{l}0.8249 \\
0.0884\end{array}$ \\
\hline $\begin{array}{l}\lambda_{9} \\
a_{9}\end{array}$ & & & & & & & $\begin{array}{r}0.9675 \\
-.0032\end{array}$ & $\begin{array}{l}0.9136 \\
0.0256\end{array}$ \\
\hline $\begin{array}{l}\lambda_{1} \\
a_{1}\end{array}$ & & & & & & & & $\begin{array}{r}0.9727 \\
-.0043\end{array}$ \\
\hline $\begin{array}{c}\boldsymbol{K}_{1} \\
\boldsymbol{h}_{2} \\
\boldsymbol{h}_{3} \\
\boldsymbol{J}\end{array}$ & $\begin{array}{l}0.6799 \\
0.8789 \\
0.0000 \\
0.4062\end{array}$ & $\begin{array}{l}0.7026 \\
0.9994 \\
0.0000 \\
0.4933\end{array}$ & $\begin{array}{l}0.7160 \\
1.0690 \\
0.0000 \\
0.5480\end{array}$ & $\begin{array}{l}0.7242 \\
1.1137 \\
0.0000 \\
0.5842\end{array}$ & $\begin{array}{l}0.7298 \\
1.1438 \\
0.0000 \\
0.6092\end{array}$ & $\begin{array}{l}0.7337 \\
1.1650 \\
0.0000 \\
0.6272\end{array}$ & $\begin{array}{l}0.7366 \\
1.1805 \\
0.0000 \\
0.6405\end{array}$ & $\begin{array}{l}0.7387 \\
1.1921 \\
0.0000 \\
0.6506\end{array}$ \\
\hline
\end{tabular}


Table A.5: Optimal Spacing for the regression and scale parameters alung with the. coefficeints for the ABLUE of $t$-distribution with $p=1$.

\begin{tabular}{|c|c|c|c|c|c|c|c|c|}
\hline \multirow[b]{2}{*}{$k$} & \multicolumn{8}{|c|}{ D.F. $=10$} \\
\hline & 3 & 4 & 5 & 6 & 7 & 8 & 9 & 10 \\
\hline$\lambda_{1}$ & 0.1270 & 0.0830 & 0.0583 & $0.042 y$ & 0.0327 & 0.0256 & $0.0204 i$ & 0.0169 \\
\hline$a_{1}$ & 0.2165 & 0.1045 & $0.05 i 1$ & 0.0332 & 0.0202 & $0.012 i$ & 0.001:-2 & 0.005 .1 \\
\hline$\lambda_{2}$ & 0.5000 & 0.3190 & 0.2214 & 0.1615 & $0.1226 ;$ & 0.0958 & o.ontis & 0.0Mi2s \\
\hline$a_{2}$ & 0.5669 & 0.3951 & 0.2477 & 0.1571 & 0.1034 & $0.070 ;$ & 0.0.49: & 0.018506 \\
\hline$\lambda_{3}$ & 0.8730 & 0.6810 & 0.5000 & 0.3692 & 0.28066 & 0.2192 & 0.175 .4 & $0.14: 31$ \\
\hline$a_{3}$ & 0.2165 & 0.3951 & 0.3904 & 0.3098 & 0.2273 & 0.1645 & 0.1203 & (0.115:03 \\
\hline$\lambda_{1}$ & & 0.9170 & 0.7786 & 0.6308 & 0.5000 & 0.3971 & 0.3197 & (0.2(i): \\
\hline$a_{4}$ & & 0.1049 & 0.2477 & 0.3098 & 0.2981 & 0.25283 & 0.2011 .1 & ה \\
\hline$\lambda_{5}$ & & & 0.9417 & 0.8385 & 0.7194 & 0.6029 & 0.50100 & (0.1151 \\
\hline$a_{5}$ & & & 0.0571 & 0.1571 & 0.2273 & 0.2523 & 0.2413 & (1.21:20) \\
\hline$\lambda_{6}$ & & & & 0.9571 & 0.8774 & $0.780 x$ & $0.6 \times 013$ & $0.5 \times 19$ \\
\hline$a_{6}$ & & & & 0.0332 & 0.1034 & 0.1645 & 0.2011 .1 & $0.21: 21$ \\
\hline$\lambda_{7}$ & & & & & 0.9673 & 0.9042 & $0.8: .16$ & 0.735 .5 \\
\hline$a_{7}$ & & & & & 0.0202 & 0.0705 & 0.1203 & (0.15) \\
\hline$\lambda_{8}$ & & & & & & 0.974. & 0.9232 & O.spotig? \\
\hline$a_{8}$ & & & & & & 0.0127 & 0.0.49: & $0.08 \div 13$ \\
\hline$\lambda_{9}$ & & & & & & & 0.9794 & $0.937: 2$ \\
\hline$a_{9}$ & & & & & & & $0.001 \times 2$ & (0.0:35x \\
\hline$\lambda_{10}$ & & & & & & & & 0.98 .31 \\
\hline-10 & & & & & & & & O.(4)ind \\
\hline$K$ & 0.7568 & 0.78 & 0.8033 & 0.8139 & 0.8210 & 0.8260 & $0.8296 ;$ & $0.532: 3$ \\
\hline$K_{2}$ & 1.0408 & 1.1981 & 1.2871 & 1.3469 & 1.3875 & 1.4167 & 1.4381 & $1.45,44$ \\
\hline 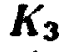 & 0.0000 & 0.0000 & 0.0000 & 0.0000 & 0.0000 & 0.0000 & 0.00000 & 0.0010) \\
\hline & 0.5962 & 0.7390 & 0.830 .5 & 0.8922 & 0.93533 & 0.9665 & 0.9898 & 1.00076 \\
\hline
\end{tabular}


Table A.6: Optimal Spacing for the regression and scale parameters along with the roefficeints for the ABLUE of $t$-distribution with $p=1$.

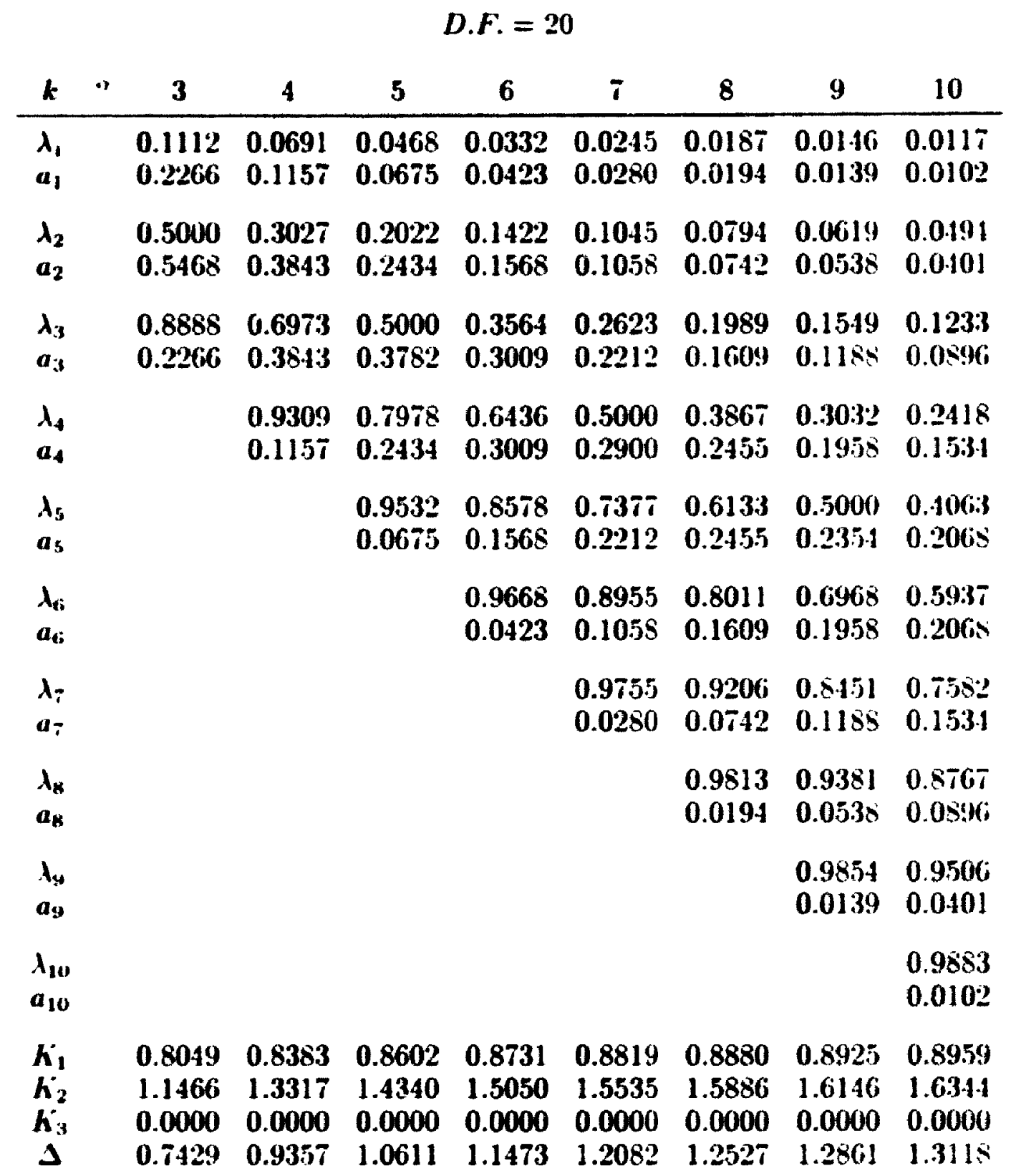


Table A.7: Optimal Spacing for the regression and scale parantelers along with the roefficeints for the ABLIE of 1 -distribution with $p=1$.

$$
\text { D.F. }=5(10
$$

\begin{tabular}{|c|c|c|c|c|c|c|c|c|}
\hline$k$ & 3 & 4 & 5 & 6 & 7 & 8 & 9 & 10 \\
\hline$\lambda_{1}$ & 0.0952 & 0.05 .54 & 0.0357 & 0.0242 & $0.01 i 1$ & 0.0126 & 0.00095 & 0.0013 \\
\hline$a_{1}$ & 0.2365 & 0.1254 & 0.0773 & 0.0507 & 0.0352 & 0.0255 .1 & $0.0 \mathrm{~s}(90)$ & $0.01118 ;$ \\
\hline$\lambda_{2}$ & 0.5000 & 0.2538 & 0.1811 & 0.1215 & $0.085 i$ & $0.062 \%$ & 0.0 .472 & U.uisti:, \\
\hline$a_{2}$ & 0.5270 & 0.37 .11 & 0.2396 & 0.1569 & 0.10 הi: & 0.0779 & $0.05 \times 00$ & $0.0111: 8$ \\
\hline$\lambda_{3}$ & 0.9048 & 0.7162 & 0.5000 & 0.3411 & 0.2411 & 0.1761 & $0.132 \cdot .1$ & 0.10101 \\
\hline$a_{::}$ & 0.236 .3 & $0.37+1$ & 0.3662 & 0.292 .4 & 0.215 .1 & 0.1570 & $0.117 \pi$ & 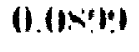 \\
\hline$\lambda_{4}$ & & 0.9446 & 0.8189 & 0.6589 & 0.5000 & $0.373 ! 9$ & $0.2 \times 34$ & $0.21 ! M 1$ \\
\hline$a_{4}$ & & 0.1254 & 0.2396 & $0.29 \div 24$ & $0.2 \times \times 2$ & $0.23: 11$ & 0.15014 & 0.1 .1918 \\
\hline$\lambda_{s}$ & & & $0.96+3$ & 0.8785 & 0.7589 & 0.6261 & 0.50000 & 0.3495 .8 \\
\hline$a s$ & & & 0.0773 & 0.1569 & 0.2151 & 0.2391 & $0.2: 294$ & 0.2019 \\
\hline$A_{f s}$ & & & & 0.9758 & 0.9143 & 0.82349 & 0.7160 & 0.6(1)17 \\
\hline$a_{*}$ & & & & 0.0507 & 0.1083 & 0.1576 & 0.19011 & 0.201019 \\
\hline$A=$ & & & & & 0.9829 & 0.9373 & o.k6ijti & 0.7810 \\
\hline$a=$ & & & & & 0.03552 & 0.0779 & 0.1177 & 0.1 .1938 \\
\hline$\lambda_{k}$ & & & & & & $0.9 \times 71$ & איק:10.95 & 0.8974 \\
\hline$a_{8}$ & & & & & & $0.025,4$ & $0.05 \pi(1)$ & (.) (18.09 \\
\hline$\lambda_{y}$ & & & & & & & 0.900: & $0.996 ; 35)$ \\
\hline$a_{\sharp}$ & & & & & & & 0.01900 & 0.04113 \\
\hline$\lambda_{10}$ & & & & & & & & i \\
\hline$a_{10}$ & & & & & & & & 0.0146 \\
\hline $\boldsymbol{K}_{\mathbf{I}}$ & 0.8597 & 0.8995 & 0.9274 & 0.9436 & 0.9549 & $0.962 x$ & 0.9687 & 0.9731 \\
\hline$K_{2}$ & 1.2705 & 1.4921 & $1.610 ;$ & 1.6966 & $1 \therefore: 50$ & 1.7981 & 1.83012 & $1.85,4 !$ \\
\hline $\boldsymbol{K}_{3}$ & 0.0000 & 0.0000 & 0.0000 & 0.0000 & 0.0000 & 0.0000 & $0.000(0)$ & О.(1)(н)(1) \\
\hline$\Delta$ & 0.9389 & 1.2074 & 1.38 .51 & 1.5106 & 1.6001 .1 & $1.666 !)$ & 1.7173 & $1.75,6.8$ \\
\hline
\end{tabular}


Appendix A

t-Dist. (Regression Parameters, $\mathbf{p = 2}$ ) 
Table A.1: Optimal Spacing for the regression and scale parameters along with the coefficeints for the ABLUE of $t$-distribution with $p=$.

\begin{tabular}{|c|c|c|c|c|c|c|c|}
\hline \multicolumn{8}{|c|}{ D.F. $=1$} \\
\hline$k$ & 4 & 5 & 6 & 7 & 8 & 9 & 10 \\
\hline $\begin{array}{l}\lambda_{1} \\
a_{1}\end{array}$ & $\begin{array}{r}0.1676 \\
-.0701\end{array}$ & $\begin{array}{r}0.1444 \\
-.0660\end{array}$ & $\begin{array}{r}0.1276 \\
-.0561\end{array}$ & $\begin{array}{r}0.1098 \\
-.0414\end{array}$ & $\begin{array}{r}0.0958 \\
-.0296\end{array}$ & $\begin{array}{l}0.0 \times 66 i \\
-.02066\end{array}$ & $\begin{array}{l}0.07 ! 91 \\
-.0176\end{array}$ \\
\hline $\begin{array}{l}\lambda_{2} \\
a_{2}\end{array}$ & $\begin{array}{l}0.4133 \\
0.5701\end{array}$ & $\begin{array}{l}0.3556 \\
0.2778\end{array}$ & $\begin{array}{l}0.3049 \\
0.1049\end{array}$ & $\begin{array}{l}0.2500 \\
0.0000\end{array}$ & $\begin{array}{r}0.2105 \\
-.0372\end{array}$ & $\begin{array}{l}0.1 \times 6 i 7 \\
-.0 .460\end{array}$ & $\begin{array}{l}0.168101 \\
-.01555\end{array}$ \\
\hline $\begin{array}{l}\lambda_{3} \\
a_{3}\end{array}$ & $\begin{array}{l}0.5867 \\
0.5701\end{array}$ & $\begin{array}{l}0.5000 \\
0.5764\end{array}$ & $\begin{array}{l}0.4409 \\
0.4512\end{array}$ & $\begin{array}{l}0.3902 \\
0.3207\end{array}$ & $\begin{array}{l}0.3 \cdot 1822 \\
0.1957\end{array}$ & $\begin{array}{l}0.313313 \\
0.111 .11\end{array}$ & $\begin{array}{l}11.2 \times 017 \\
0.010 .5\end{array}$ \\
\hline $\begin{array}{l}\lambda_{4} \\
a_{4}\end{array}$ & $\begin{array}{l}0.8324 \\
-.0701\end{array}$ & $\begin{array}{l}0.6444 \\
0.2778\end{array}$ & $\begin{array}{l}0.5591 \\
0.4512\end{array}$ & $\begin{array}{l}0.5000 \\
0.4414\end{array}$ & $\begin{array}{l}0.452 .3 \\
0.3711\end{array}$ & $\begin{array}{l}0.413 .14 \\
0.2908\end{array}$ & $\begin{array}{l}0.3791 \\
0.217 ! 1\end{array}$ \\
\hline $\begin{array}{l}\lambda_{5} \\
a_{5}\end{array}$ & & $\begin{array}{l}0.8556 \\
-.0660\end{array}$ & $\begin{array}{l}0.6951 \\
0.1049\end{array}$ & $\begin{array}{l}0.6098 \\
0.2207\end{array}$ & $\begin{array}{l}0.5477 \\
0.3711\end{array}$ & $\begin{array}{l}0.5000 \\
0.3473\end{array}$ & $\begin{array}{l}0.46113 \\
0.3010\end{array}$ \\
\hline $\begin{array}{l}\lambda_{6} \\
a_{6}\end{array}$ & & & $\begin{array}{l}0.8724 \\
-.0561\end{array}$ & $\begin{array}{l}0.7500 \\
0.0000\end{array}$ & $\begin{array}{l}0.6518 \\
0.1957\end{array}$ & $\begin{array}{l}0.5 \times 666 \\
0.2906\end{array}$ & $\begin{array}{l}0.5 .3 \times 17 \\
0.30 .17\end{array}$ \\
\hline $\begin{array}{l}\lambda_{7} \\
a_{7}\end{array}$ & & & & $\begin{array}{l}0.8902 \\
-.0414\end{array}$ & $\begin{array}{c}0.7895 \\
-.0372\end{array}$ & $\begin{array}{l}0.6 \times 6 i 7 \\
0.1011\end{array}$ & $\begin{array}{l}0.6206 \\
0.2179\end{array}$ \\
\hline $\begin{array}{l}\lambda_{8} \\
a_{8}\end{array}$ & & & & & $\begin{array}{r}0.9042 \\
-.0296\end{array}$ & $\begin{array}{l}0.8133 \\
-.0460\end{array}$ & $\begin{array}{l}0.7193 \\
0.0405\end{array}$ \\
\hline $\begin{array}{l}\lambda_{9} \\
a_{9}\end{array}$ & & & & & & $\begin{array}{r}0.9131 \\
-.0226\end{array}$ & $\begin{array}{l}0.831 ! ! \\
-.045 !)\end{array}$ \\
\hline $\begin{array}{l}\lambda_{10} \\
a_{10}\end{array}$ & & & & & & & $\begin{array}{l}0.920 ! 9 \\
-.0177\end{array}$ \\
\hline$K_{1}$ & 0.4530 & 0.4675 & 0.4734 & 0.4782 & $0.4 \times 332$ & (1. $4 \times(.5)$ & $0.4 \sin \theta$ \\
\hline$K_{2}$ & 0.4106 & 0.4355 & 0.4566 & 0.4693 & 0.4744 & 0.4790 & 0.48 .30 \\
\hline$K_{3}$ & 0.0000 & 0.0000 & 0.0000 & 0.0000 & 0.0000 & 0.00000 & 0.001000 \\
\hline$\Delta$ & 0.0382 & 0.0445 & 0.0484 & 0.0513 & 0.05355 & 0.0551 & (0.0), (6.3) \\
\hline
\end{tabular}


Table A.2: Optimal Spacing for the regression and scale parameters along with the coefficeints for the ABLUE of $t$-distribution with $p=2$.

\begin{tabular}{|c|c|c|c|c|c|c|c|}
\hline \multicolumn{8}{|c|}{$D . F .=2$} \\
\hline$k$ & 4 & 5 & 6 & 7 & 8 & 9 & 10 \\
\hline $\begin{array}{l}\lambda_{1} \\
a_{1}\end{array}$ & $\begin{array}{l}0.1651 \\
0.0168\end{array}$ & $\begin{array}{l}0.1194 \\
-.0254\end{array}$ & $\begin{array}{c}0.0924 \\
-.0326\end{array}$ & $\begin{array}{r}0.0748 \\
-.0305\end{array}$ & $\begin{array}{l}0.0622 \\
-.0261\end{array}$ & $\begin{array}{c}0.0527 \\
-.0216\end{array}$ & $\begin{array}{c}\mathbf{0 . 0 4 5 4} \\
-.0176\end{array}$ \\
\hline $\begin{array}{l}\lambda_{2} \\
a_{2}\end{array}$ & $\begin{array}{l}0.3920 \\
0.4832\end{array}$ & $\begin{array}{l}0.3146 \\
0.2886\end{array}$ & $\begin{array}{l}0.2576 \\
0.1564\end{array}$ & $\begin{array}{l}0.2136 \\
0.0755\end{array}$ & $\begin{array}{l}0.1786 \\
0.0284\end{array}$ & $\begin{array}{l}0.1510 \\
0.0026\end{array}$ & $\begin{array}{r}0.1293 \\
-.0105\end{array}$ \\
\hline $\begin{array}{l}\lambda_{3} \\
a_{3}\end{array}$ & $\begin{array}{l}0.6080 \\
0.4832\end{array}$ & $\begin{array}{l}0.5000 \\
0.4735\end{array}$ & $\begin{array}{l}0.4208 \\
0.3762\end{array}$ & $\begin{array}{l}0.3599 \\
0.2776\end{array}$ & & & $\begin{array}{l}0.2370 \\
0.08 .10\end{array}$ \\
\hline $\begin{array}{l}\lambda_{4} \\
a_{4}\end{array}$ & $\begin{array}{l}0.8349 \\
0.0168\end{array}$ & $\begin{array}{l}0.6854 \\
0.2886\end{array}$ & $\begin{array}{l}0.5792 \\
0.3762\end{array}$ & $\begin{array}{l}0.5000 \\
0.3547\end{array}$ & & $\begin{array}{l}0.3871 \\
0.2451\end{array}$ & $\begin{array}{l}0.3448 \\
0.1925\end{array}$ \\
\hline $\begin{array}{l}\lambda_{5} \\
a_{5}\end{array}$ & & $\begin{array}{l}0.8806 \\
-.0254\end{array}$ & $\begin{array}{l}0.7424 \\
0.1564\end{array}$ & $\begin{array}{l}0.6401 \\
0.2776\end{array}$ & & $\begin{array}{l}0.5000 \\
0.2847\end{array}$ & $\begin{array}{l}0.4487 \\
0.2517\end{array}$ \\
\hline $\begin{array}{l}\lambda_{6} \\
a_{6}\end{array}$ & & & $\begin{array}{r}0.9076 \\
-.0326\end{array}$ & & & & $\begin{array}{l}0.5513 \\
0.2517\end{array}$ \\
\hline $\begin{array}{l}\lambda_{7} \\
a_{7}\end{array}$ & & & & $\begin{array}{l}0.9252 \\
-.0305\end{array}$ & $\begin{array}{l}0.8214 \\
0.0284\end{array}$ & $\begin{array}{l}0.7294 \\
0.1316\end{array}$ & $\begin{array}{l}0.6552 \\
0.1925\end{array}$ \\
\hline $\begin{array}{l}\lambda_{8} \\
a_{8}\end{array}$ & & & & & $\begin{array}{l}0.9378 \\
-.0261\end{array}$ & $\begin{array}{l}0.8490 \\
0.0026\end{array}$ & $\begin{array}{l}0.7630 \\
0.0840\end{array}$ \\
\hline $\begin{array}{l}\lambda_{9} \\
a_{9}\end{array}$ & & & & & & $\begin{array}{l}0.9473 \\
-.0216\end{array}$ & $\begin{array}{l}0.8707 \\
-.0105\end{array}$ \\
\hline $\begin{array}{l}\lambda_{10} \\
a_{10}\end{array}$ & & & & & & & $\begin{array}{l}0.9546 \\
-.0176\end{array}$ \\
\hline $\begin{array}{l}\boldsymbol{K}_{1} \\
\boldsymbol{K}_{2} \\
\boldsymbol{K}_{3} \\
\boldsymbol{\Delta}\end{array}$ & $\begin{array}{l}0.5534 \\
0.6682 \\
0.0000 \\
0.1132\end{array}$ & $\begin{array}{l}0.5666 \\
0.7050 \\
0.0000 \\
0.1262\end{array}$ & $\begin{array}{l}0.5752 \\
0.7274 \\
0.0000 \\
0.138\end{array}$ & $\begin{array}{l}0.5807 \\
0.7437 \\
0.0000 \\
0.1456\end{array}$ & $\begin{array}{l}0.5844 \\
0.7553 \\
0.0000 \\
0.1508\end{array}$ & $\begin{array}{l}0.5872 \\
0.7636 \\
0.0000 \\
0.1546\end{array}$ & $\begin{array}{l}0.5894 \\
0.7698 \\
0.0000 \\
0.1576\end{array}$ \\
\hline
\end{tabular}


Table A.3: Optimal Spacing for the regression and scale parameters along with the coefficeints for the ABLUE of $t$-distribution with $p=2$.

$$
\text { D.F. }=3
$$

\begin{tabular}{|c|c|c|c|c|c|c|c|}
\hline$k$ & 4 & 5 & 6 & 7 & 8 & 9 & 10 \\
\hline $\begin{array}{l}\lambda_{1} \\
a_{1}\end{array}$ & $\begin{array}{l}0.1472 \\
0.0640\end{array}$ & $\begin{array}{l}0.1098 \\
0.0124\end{array}$ & $\begin{array}{r}0.0843 \\
-.0078\end{array}$ & $\begin{array}{r}0.0665 \\
-.0146\end{array}$ & $\begin{array}{c}0.0539) \\
-.0160\end{array}$ & $\begin{array}{r}0.0 \cdot 446 \\
-.0152\end{array}$ & $\begin{array}{l}0.0375 \\
-.0137\end{array}$ \\
\hline $\begin{array}{l}\lambda_{2} \\
a_{2}\end{array}$ & $\begin{array}{l}0.3774 \\
0.4360\end{array}$ & $\begin{array}{l}0.2953 \\
0.2770\end{array}$ & $\begin{array}{l}0.2365 \\
0.1688\end{array}$ & $\begin{array}{l}0.1930 \\
0.0995\end{array}$ & $\begin{array}{l}0.1599 \\
0.0561\end{array}$ & $\begin{array}{l}0.134 .4 \\
0.0293\end{array}$ & $\begin{array}{l}0.11 .13 \\
0.01330\end{array}$ \\
\hline $\begin{array}{l}\lambda_{3} \\
a_{3}\end{array}$ & $\begin{array}{l}6226 \\
4360\end{array}$ & & & & $\begin{array}{l}0.2907 \\
0.1867\end{array}$ & $\begin{array}{l}0.2489 \\
0.1337\end{array}$ & $\begin{array}{l}0.215 \% 2 \\
0.09 .1 .2\end{array}$ \\
\hline $\begin{array}{l}\lambda_{4} \\
a_{4}\end{array}$ & & & & & & & $\begin{array}{l}0.32660 \\
0.17 \times 6\end{array}$ \\
\hline $\begin{array}{l}\lambda_{5} \\
a_{5}\end{array}$ & & $\begin{array}{l}0.8902 \\
0.0124\end{array}$ & $\begin{array}{l}0.7635 \\
0.1688\end{array}$ & & $\begin{array}{l}0.5704 \\
0.2732\end{array}$ & $\begin{array}{l}0.5000 \\
0.2571\end{array}$ & $\begin{array}{l}0.441 ! \\
0.2: 27 !\end{array}$ \\
\hline $\begin{array}{l}\lambda_{6} \\
a_{6}\end{array}$ & & & & & & & $\begin{array}{l}0.5151 \\
0.2279\end{array}$ \\
\hline $\begin{array}{l}\lambda_{7} \\
a_{7}\end{array}$ & & & & $\begin{array}{r}0.9335 \\
-.0146\end{array}$ & $\begin{array}{l}0.8401 \\
0.0561\end{array}$ & $\begin{array}{l}0.7511 \\
0.1337\end{array}$ & $\begin{array}{l}0.67335 \\
0.1786\end{array}$ \\
\hline $\begin{array}{l}\lambda_{8} \\
a_{8}\end{array}$ & & & & & $\begin{array}{l}0.9461 \\
-.0160\end{array}$ & $\begin{array}{l}0.8656 \\
0.0293\end{array}$ & $\begin{array}{l}0.7848 \\
0.09942\end{array}$ \\
\hline $\begin{array}{l}\lambda_{9} \\
a_{9}\end{array}$ & & & & & & $\begin{array}{r}0.9554 \\
-.0152\end{array}$ & $\begin{array}{l}0.85: 7 \\
0.01330\end{array}$ \\
\hline $\begin{array}{l}\lambda_{10} \\
a_{10}\end{array}$ & & & & & & & $\begin{array}{l}0.96225) \\
-.0137\end{array}$ \\
\hline $\begin{array}{c}K_{1} \\
K_{2} \\
K_{3} \\
\Delta\end{array}$ & $\begin{array}{l}0.6262 \\
0.8089 \\
0.0000 \\
0.1986\end{array}$ & $\begin{array}{l}0.6363 \\
0.8660 \\
0.0000 \\
0.2231\end{array}$ & $\begin{array}{l}0.6432 \\
0.9005 \\
0.0000 \\
0.2397\end{array}$ & $\begin{array}{l}0.6482 \\
0.9229 \\
0.0000 \\
0.2513\end{array}$ & $\begin{array}{l}0.6517 \\
0.9385 \\
0.0000 \\
0.2598\end{array}$ & $\begin{array}{l}0.6544 \\
0.9498 \\
0.0000 \\
0.2661\end{array}$ & $\begin{array}{l}0.65644 \\
0.95882 \\
0.0000 \\
0.2710\end{array}$ \\
\hline
\end{tabular}


Table A.4: Optimal Spacing for the regression and scale parameters along with the coefficeints for the ABLUE of $t$-distribution with $p=2$.

\begin{tabular}{cccccccc}
\multicolumn{7}{c}{$D . F .=5$} \\
$k$ & 4 & 5 & 6 & 7 & 8 & 9 & 10 \\
\hline$\lambda_{1}$ & 0.1196 & 0.0883 & 0.0676 & 0.0532 & 0.0429 & 0.0352 & 0.0294 \\
$a_{1}$ & 0.0963 & 0.0444 & 0.0196 & 0.0072 & 0.0009 & -.0023 & -.0038 \\
$\lambda_{2}$ & 0.3572 & 0.2679 & 0.2080 & 0.1659 & 0.1351 & 0.1120 & 0.0943 \\
$a_{2}$ & 0.4037 & 0.2621 & 0.1691 & 0.1099 & 0.0721 & 0.0476 & 0.0314 \\
$\lambda_{3}$ & 0.6428 & 0.5000 & 0.3969 & 0.3215 & 0.2651 & 0.2219 & 0.1882 \\
$a_{3}$ & 0.4037 & 0.3871 & 0.3114 & 0.2365 & 0.1763 & 0.1308 & 0.0972 \\
$\lambda_{4}$ & 0.8804 & 0.7321 & 0.6031 & 0.5000 & 0.4190 & 0.3550 & 0.3040 \\
$a_{4}$ & 0.0963 & 0.2621 & 0.3114 & 0.2928 & 0.2507 & 0.2060 & 0.1660 \\
$\lambda_{5}$ & & 0.9117 & 0.7920 & 0.6785 & 0.5810 & 0.5000 & 0.4331 \\
$a_{5}$ & & 0.0444 & 0.1691 & 0.2365 & 0.2507 & 0.2359 & 0.2093 \\
$\lambda_{6}$ & & & 0.9324 & 0.8341 & 0.7349 & 0.6450 & 0.5669 \\
$a_{6}$ & & & 0.0196 & 0.1099 & 0.1763 & 0.2060 & 0.2093 \\
$\lambda_{7}$ & & & & 0.9468 & 0.8649 & 0.7781 & 0.6960 \\
$a_{7}$ & & & & 0.0072 & 0.0721 & 0.1308 & 0.1660 \\
$\lambda_{8}$ & & & & & 0.9571 & 0.8880 & 0.8118 \\
$a_{8}$ & & & & & 0.0009 & 0.0476 & 0.0972 \\
$\lambda_{9}$ & & & & & & 0.9648 & 0.9057 \\
$a_{9}$ & & & & & & -.0023 & 0.0314 \\
$\lambda_{10}$ & & & & & & & 0.9706 \\
$a_{10}$ & & & & & & & -.0038 \\
$K_{1}$ & 0.7076 & 0.7192 & 0.7265 & 0.7315 & 0.7350 & 0.7376 & 0.7396 \\
$\boldsymbol{h}_{2}$ & 0.9820 & 1.0571 & 1.1050 & 1.1373 & 1.1599 & 1.1763 & 1.1887 \\
$\boldsymbol{K}_{3}$ & 0.0000 & 0.0000 & 0.0000 & 0.0000 & 0.0000 & 0.0000 & 0.0000 \\
$\Delta$ & 0.3479 & 0.3933 & 0.4238 & 0.4452 & 0.4606 & 0.4721 & 0.4809
\end{tabular}


Table A.5: Optimal Spacing for the regression and scale parameters along with the coefficeints for the ABLUE of $t$-distribution with $\mu=2$.

\begin{tabular}{|c|c|c|c|c|c|c|c|}
\hline \multicolumn{8}{|c|}{$D . F .=10$} \\
\hline$k$ & 4 & 5 & 6 & 7 & 8 & 9 & 111 \\
\hline$\lambda_{1}$ & 0.0923 & 0.0652 & 0.0481 & 0.0368 & 0.0289 & 0.0233 & $0.019 !$ \\
\hline$a_{1}$ & 0.1175 & 0.0659 & 0.0392 & 0.0244 & 0.0157 & 0.0103 & $0.00(6)$ \\
\hline$\lambda_{2}$ & $\mathbf{0 . 3 3 4 1}$ & 0.2368 & 0.1754 & 0.1344 & 0.1050 & 0.085is & 0.0700 \\
\hline$a_{2}$ & 0.3825 & 0.2510 & 0.1663 & 0.1131 & 0.0790 & 0.05666 & 0.01113 \\
\hline$\lambda_{3}$ & 0.6659 & 0.5000 & 0.3798 & 0.2948 & 0.2340 & 0.189 .1 & 0.15 .59 \\
\hline$a_{3}$ & 0.3825 & 0.3662 & 0.2944 & 0.2237 & 0.1679 & $0.126(j$ & $0.094(1)$ \\
\hline$\lambda_{4}$ & 0.9077 & 0.7632 & 0.6202 & 0.5000 & 0.4053 & $0.33: 3$ & 0.2757 \\
\hline$a_{4}$ & 0.1175 & 0.2510 & 0.2944 & 0.2775 & 0.237 .4 & $0.1911 i$ & 0.1570 \\
\hline$\lambda_{5}$ & & 0.9348 & 0.8246 & 0.7052 & 0.5947 & 0.5000 & 0.4218 \\
\hline$a_{5}$ & & 0.0659 & 0.1663 & 0.2237 & 0.2374 & 0.2238 & $0.1 ! 183$ \\
\hline$\lambda_{6}$ & & & 0.9519 & 0.8656 & 0.7660 & 0.6675 & $0.57 \times 2$ \\
\hline$a_{6}$ & & & 0.0392 & 0.1131 & 0.1679 & 0.1946 & 0.19.8.3 \\
\hline$\lambda_{7}$ & & & & 0.9632 & 0.8941 & 0.8106 & $0.7 \div 913$ \\
\hline$a_{7}$ & & & & 0.0244 & 0.0790 & $0.126 i$ & 0.1570 \\
\hline$\lambda_{B}$ & & & & & 0.9711 & 0.9147 & 0.8 .111 \\
\hline$a_{8}$ & & & & & 0.0157 & 0.05660 & 0.0966 .4 \\
\hline$\lambda_{9}$ & & & & & & 0.9767 & 0.93300 \\
\hline$a_{9}$ & & & & & & 0.0103 & 0.0413 \\
\hline$\lambda_{10}$ & & & & & & & 0.9809 \\
\hline$a_{10}$ & & & & & & & $0.006,9$ \\
\hline$K_{1}$ & 0.7911 & 0.8069 & 0.8167 & 0.8232 & 0.8277 & 0.8 .110 & 0.8.3.35 \\
\hline$K_{2}$ & 1.1771 & 1.2729 & 1.3357 & 1.3788 & 1.4095 & 1.4323 & 1.4495 \\
\hline$K_{3}$ & 0.0000 & 0.0000 & 0.0000 & 0.0000 & 0.0000 & 0.00000 & $0.00(0) 0$ \\
\hline$\Delta$ & 0.5828 & 0.6687 & 0.7275 & 0.7690 & 0.7993 & 0.8219 & $0.83 \% 3$ \\
\hline
\end{tabular}


Table A.6: Optimal Spacing for the regression and scale parameters along with the coefficeints for the ABLUE of t-distribution with $p=2$.

\begin{tabular}{cccccccc}
\multicolumn{8}{c}{$D . F .=20$} \\
$k$ & 4 & 5 & 6 & 7 & 8 & 9 & 10 \\
\hline$\lambda_{1}$ & 0.0771 & 0.0523 & $j .0373$ & 0.0277 & 0.0212 & 0.0166 & 0.0133 \\
$a_{1}$ & 0.1273 & 0.0757 & 0.0482 & 0.0323 & 0.0225 & 0.0162 & 0.0120 \\
$\lambda_{2}$ & 0.3188 & 0.2170 & 0.1549 & 0.1150 & 0.0880 & 0.0690 & 0.0553 \\
$a_{2}$ & 0.3727 & 0.2461 & 0.1649 & 0.1143 & 0.0817 & 0.0601 & 0.0453 \\
$\lambda_{3}$ & 0.6812 & 0.5000 & 0.3678 & 0.2766 & 0.2131 & 0.1679 & 0.1348 \\
$a_{3}$ & 0.3727 & 0.3565 & 0.2869 & 0.2179 & 0.1640 & 0.1244 & 0.0958 \\
& & & & & & & \\
$\lambda_{4}$ & 0.9229 & 0.7830 & 0.6322 & 0.5000 & 0.3955 & 0.3161 & 0.2560 \\
$a_{4}$ & 0.1273 & 0.2461 & 0.2869 & 0.2710 & 0.2318 & 0.1897 & 0.1529 \\
$\lambda_{5}$ & & 0.9477 & 0.8451 & 0.7234 & 0.6045 & 0.5000 & 0.4135 \\
$a_{5}$ & & 0.0757 & 0.1649 & 0.2179 & 0.2318 & 0.2191 & 0.1940 \\
$\lambda_{6}$ & & & 0.9627 & 0.8850 & 0.7869 & 0.6839 & 0.5865 \\
$a_{6}$ & & & 0.0482 & 0.1143 & 0.1640 & 0.1897 & 0.1940 \\
$\lambda_{7}$ & & & & 0.9723 & 0.9120 & 0.8321 & 0.7440 \\
$a_{7}$ & & & & 0.0323 & 0.0817 & 0.1244 & 0.1529 \\
$\lambda_{8}$ & & & & & 0.9788 & 0.9310 & 0.8652 \\
$a_{8}$ & & & & & 0.0225 & 0.0601 & 0.0958 \\
$\lambda_{9}$ & & & & & & 0.9834 & 0.9447 \\
$a_{9}$ & & & & & & 0.0162 & 0.0453 \\
$\lambda_{10}$ & & & & & & & 0.9867 \\
$a_{10}$ & & & & & & & 0.0120 \\
$\boldsymbol{K}_{1}$ & 0.8443 & 0.8639 & 0.8760 & 0.8841 & 0.8898 & 0.8940 & 0.8971 \\
$\boldsymbol{K}_{2}$ & 1.3086 & 1.4192 & 1.4929 & 1.5440 & 0.5808 & 1.6081 & 1.6290 \\
$K_{3}$ & 0.0000 & 0.0000 & 0.0000 & 0.0000 & 0.0000 & 0.0000 & 0.0000 \\
$\Delta$ & 0.7876 & 0.9149 & 1.0036 & 1.0670 & 1.1137 & 1.1489 & 1.1761
\end{tabular}


Table A.7: Optimal Spacing for the regression and scale parameters along with the. coefficeints for the ABLUE of $t$-distribution with $p=2$.

\begin{tabular}{|c|c|c|c|c|c|c|c|}
\hline \multicolumn{8}{|c|}{ D.F. $=500$} \\
\hline $\boldsymbol{k}$ & 4 & 5 & 6 & 7 & 8 & 9 & 10 \\
\hline $\begin{array}{l}\lambda_{1} \\
a_{1}\end{array}$ & $\begin{array}{l}0.0619 \\
0.1365\end{array}$ & $\begin{array}{l}0.0400 \\
0.0847\end{array}$ & $\begin{array}{l}0.0272 \\
0.0562\end{array}$ & $\begin{array}{l}0.0193 \\
0.0393\end{array}$ & $\begin{array}{l}0.0142 \\
0.0285\end{array}$ & $\begin{array}{l}0.0108 \\
0.021 .4\end{array}$ & $\begin{array}{l}0.0083 \\
0.016 i 5\end{array}$ \\
\hline $\begin{array}{ll}\lambda_{2} & \\
a_{2} & 0 .\end{array}$ & $\begin{array}{l}0.3007 \\
0.3635\end{array}$ & $\begin{array}{l}0.1947 \\
0.2417\end{array}$ & $\begin{array}{l}0.1327 \\
0.1639\end{array}$ & $\begin{array}{l}0.0944 \\
0.1155\end{array}$ & $\begin{array}{l}0.0695 \\
0.08 .43\end{array}$ & $\begin{array}{l}0.052 \overline{1} \\
0.0633\end{array}$ & $\begin{array}{l}0.0 .108 \\
0.0 .18 \times\end{array}$ \\
\hline $\begin{array}{l}\lambda_{3} \\
a_{3}\end{array}$ & $\begin{array}{l}0.6993 \\
0.3635\end{array}$ & $\begin{array}{l}0.5000 \\
0.3472\end{array}$ & & & $\begin{array}{l}0.1892 \\
0.160 .1\end{array}$ & $\begin{array}{l}0.1439 \\
0.1226\end{array}$ & $\begin{array}{l}0.1118 \\
0.0952\end{array}$ \\
\hline $\begin{array}{l}\lambda_{4} \\
a_{4}\end{array}$ & $\begin{array}{l}0.9381 \\
0.1365\end{array}$ & $\begin{array}{l}0.8053 \\
0.2417\end{array}$ & $\begin{array}{l}0.6468 \\
0.2799\end{array}$ & $\begin{array}{l}0.5000 \\
0.2651\end{array}$ & $\begin{array}{l}0.3834 \\
0.2267\end{array}$ & $\begin{array}{l}0.2965 \\
0.185 \%\end{array}$ & $\begin{array}{l}0.2: 326 \\
0.1 .492\end{array}$ \\
\hline $\begin{array}{l}\lambda_{5} \\
a_{5}\end{array}$ & & $\begin{array}{l}0.9600 \\
0.0847\end{array}$ & $\begin{array}{l}0.8673 \\
0.1639\end{array}$ & & & $\begin{array}{l}0.5000 \\
0.2150\end{array}$ & $\begin{array}{l}0.4031 \\
0.190: 3\end{array}$ \\
\hline $\begin{array}{l}\lambda_{6} \\
a_{6}\end{array}$ & & & $\begin{array}{l}0.9728 \\
0.0562\end{array}$ & $\begin{array}{l}0.9056 \\
0.1155\end{array}$ & $\begin{array}{l}0.8108 \\
0.1604\end{array}$ & $\begin{array}{l}0.7035: \\
0.1852\end{array}$ & $\begin{array}{l}0.5969 \\
0.1903\end{array}$ \\
\hline $\begin{array}{l}\lambda_{7} \\
a_{7}\end{array}$ & & & & $\begin{array}{l}0.9807 \\
0.0303\end{array}$ & $\begin{array}{l}0.9305 \\
0.0843\end{array}$ & $\begin{array}{l}0.856(61 \\
0.1226\end{array}$ & $\begin{array}{l}0.7674 \\
0.1492\end{array}$ \\
\hline $\begin{array}{l}\lambda_{8} \\
a_{8}\end{array}$ & & & & & $\begin{array}{l}0.9858 \\
0.0285\end{array}$ & $\begin{array}{l}0.9473 \\
0.063 .33\end{array}$ & $\begin{array}{l}0.8852 \\
0.09522\end{array}$ \\
\hline $\begin{array}{l}\lambda_{9} \\
a_{9}\end{array}$ & & & & & & $\begin{array}{l}0.9892 \\
0.0214\end{array}$ & $\begin{array}{l}0.9592 \\
0.0488\end{array}$ \\
\hline $\begin{array}{l}\lambda_{10} \\
a_{10}\end{array}$ & & & & & & & $\begin{array}{l}0.9917 \\
0.0165\end{array}$ \\
\hline $\begin{array}{c}K_{1} \\
K_{2} \\
K_{3} \\
\Delta\end{array}$ & $\begin{array}{l}0.9060 \\
1.4666 \\
0.0000 \\
1.0905\end{array}$ & $\begin{array}{l}0.9310 \\
1.5955 \\
0.0000 \\
1.2873\end{array}$ & $\begin{array}{l}0.9465 \\
1.6837 \\
0.0000 \\
1.4279\end{array}$ & $\begin{array}{l}0.9571 \\
1.7452 \\
0.0000 \\
1.5302\end{array}$ & $\begin{array}{l}0.9646 \\
1.7899 \\
0.0000 \\
1.6066\end{array}$ & $\begin{array}{l}0.9701 \\
1.8234 \\
0.0000 \\
1.6649\end{array}$ & $\begin{array}{l}0.9743 \\
1.8491 \\
0.0000 \\
1.7102\end{array}$ \\
\hline
\end{tabular}


Appendix A

t-Dist. (Regression Parameters, $p=3$ ) 
Table A.1: Optimal Spacing for the regression and scale parameters along with the coefficeints for the ABLUE of $t$-distribution with $p=3$.

\begin{tabular}{|c|c|c|c|c|c|c|}
\hline \multirow[b]{2}{*}{$\boldsymbol{k}$} & \multicolumn{5}{|c|}{ D.F. $=1$} & \multirow[b]{2}{*}{10} \\
\hline & 5 & 6 & 7 & 8 & 9 & \\
\hline $\begin{array}{l}\lambda_{1} \\
a_{1}\end{array}$ & $\begin{array}{r}0.1377 \\
-.0610\end{array}$ & $\begin{array}{r}0.1253 \\
-.0543\end{array}$ & $\begin{array}{l}0.1071 \\
-.0397\end{array}$ & $\begin{array}{r}0.0915 \\
-.0265\end{array}$ & $\begin{array}{c}0.08: 36 \\
-.0208\end{array}$ & $\begin{array}{l}0.0772 \\
-.0166 i\end{array}$ \\
\hline $\begin{array}{l}\lambda_{2} \\
a_{2}\end{array}$ & $\begin{array}{l}0.3623 \\
0.2860\end{array}$ & $\begin{array}{l}0.3159 \\
0.1218\end{array}$ & $\begin{array}{l}0.2500 \\
0.0000\end{array}$ & $\begin{array}{l}0.2026 \\
-.0393\end{array}$ & $\begin{array}{r}0.1816 \\
-.0489\end{array}$ & $\begin{array}{r}0.165 .2 \\
-.0451\end{array}$ \\
\hline $\begin{array}{l}\lambda_{3} \\
a_{3}\end{array}$ & $\begin{array}{l}0.5000 \\
0.5500\end{array}$ & $\begin{array}{l}0.4443 \\
0.4325\end{array}$ & $\begin{array}{l}0.3929 \\
0.3242\end{array}$ & $\begin{array}{l}0.3507 \\
0.2006\end{array}$ & $\begin{array}{l}0.318 .1 \\
0.1115\end{array}$ & $\begin{array}{l}0.286(0) \\
0.016(i t i\end{array}$ \\
\hline $\begin{array}{l}\lambda_{4} \\
a_{4}\end{array}$ & $\begin{array}{l}0.6377 \\
0.2860\end{array}$ & $\begin{array}{l}0.5557 \\
0.4325\end{array}$ & $\begin{array}{l}0.5000 \\
0.4310\end{array}$ & $\begin{array}{l}0.4533 \\
0.3652\end{array}$ & $\begin{array}{l}0.416 .1 \\
02875\end{array}$ & $\begin{array}{l}0.3 \times 3737 \\
0.220107\end{array}$ \\
\hline $\begin{array}{l}\lambda_{5} \\
a_{5}\end{array}$ & $\begin{array}{r}0.8623 \\
-.0610\end{array}$ & $\begin{array}{l}0.6841 \\
0.1218\end{array}$ & $\begin{array}{l}0.6071 \\
0.3242\end{array}$ & $\begin{array}{l}0.5467 \\
0.3652\end{array}$ & $\begin{array}{l}0.5000 \\
0.3356\end{array}$ & $\begin{array}{l}0.46 .27 \\
0.29 .47\end{array}$ \\
\hline $\begin{array}{l}\lambda_{6} \\
a_{6}\end{array}$ & & $\begin{array}{l}\mathbf{0 . 8 7 4 7} \\
-.0543\end{array}$ & $\begin{array}{l}0.7500 \\
0.0000\end{array}$ & $\begin{array}{l}0.6493 \\
0.2006\end{array}$ & $\begin{array}{l}0.58 .36 \\
0.2875\end{array}$ & $\begin{array}{l}0.53 .373 \\
0.29 .47\end{array}$ \\
\hline $\begin{array}{l}\lambda_{7} \\
a_{7}\end{array}$ & & & $\begin{array}{c}0.8929 \\
-.0397\end{array}$ & $\begin{array}{l}0.7974 \\
-.0393\end{array}$ & $\begin{array}{l}0.6816 \\
0.1115\end{array}$ & $\begin{array}{l}0.61(f i 3) \\
0.22(1)\end{array}$ \\
\hline $\begin{array}{l}\lambda_{8} \\
a_{8}\end{array}$ & & & & $\begin{array}{r}0.9085 \\
-.0265\end{array}$ & $\begin{array}{l}0.8184 \\
-.0459\end{array}$ & $\begin{array}{l}0.71411 \\
0.0466\end{array}$ \\
\hline $\begin{array}{l}\lambda_{9} \\
a_{9}\end{array}$ & & & & & $\begin{array}{l}0.9164 \\
-.0208\end{array}$ & $\begin{array}{r}0.83 .38 \\
-.0454\end{array}$ \\
\hline $\begin{array}{l}\lambda_{10} \\
a_{10}\end{array}$ & & & & & & $\begin{array}{l}0.9228 \\
-.0166\end{array}$ \\
\hline $\begin{array}{c}K_{1} \\
K_{2} \\
K_{3} \\
\Delta\end{array}$ & $\begin{array}{l}0.4703 \\
0.4264 \\
0.0000 \\
0.0209\end{array}$ & $\begin{array}{l}0.4752 \\
0.4506 \\
0.0000 \\
0.0230\end{array}$ & $\begin{array}{l}0.4786 \\
0.4681 \\
0.0000 \\
0.0246\end{array}$ & $\begin{array}{l}0.4841 \\
0.4716 \\
0.0000 \\
0.0259\end{array}$ & $\begin{array}{l}0.4872 \\
0.4766 \\
0.0000 \\
0.0268\end{array}$ & $\begin{array}{l}0.4890 \\
0.4815) \\
0.000(0) \\
0.0275)\end{array}$ \\
\hline
\end{tabular}


Table A.2: Optimal Spacing the regression and scale parameters along with the coefficeints for the ABLUE of $t$-distribution with $p=3$.

\begin{tabular}{ccccccc}
\multicolumn{7}{c}{$D . F .=2$} \\
$k$ & 5 & 6 & 7 & 8 & 9 & 10 \\
\hline$\lambda_{1}$ & 0.1162 & 0.0884 & 0.0719 & 0.0599 & 0.0506 & 0.0434 \\
$a_{1}$ & -.0248 & -.0313 & -.0293 & -.0252 & -.0207 & -.0165 \\
$\lambda_{2}$ & 0.3199 & 0.2641 & 0.2204 & 0.1837 & 0.1531 & 0.1293 \\
$a_{2}$ & 0.2946 & 0.1645 & 0.0829 & 0.0327 & 0.0040 & -.0105 \\
$\lambda_{3}$ & 0.5000 & 0.4238 & 0.3654 & 0.3176 & 0.2771 & 0.2430 \\
$a_{3}$ & 0.4603 & 0.3668 & 0.2760 & 0.2002 & 0.1387 & 0.0908 \\
$\lambda_{4}$ & 0.6801 & 0.5762 & 0.5000 & 0.4404 & 0.3913 & 0.3500 \\
$a_{4}$ & 0.2946 & 0.3668 & 0.3408 & 0.2923 & 0.2409 & 0.1929 \\
$\lambda_{5}$ & 0.8838 & 0.7359 & 0.6346 & 0.5596 & 0.5000 & 0.4506 \\
$a_{5}$ & -.0248 & 0.1645 & 0.2760 & 0.2923 & 0.2742 & 0.2435 \\
$\lambda_{6}$ & & 0.9116 & 0.7796 & 0.6824 & 0.6087 & 0.5494 \\
$a_{6}$ & & -.0313 & 0.0829 & 0.2002 & 0.2409 & 0.2435 \\
$\lambda_{7}$ & & & 0.9281 & 0.8163 & 0.7229 & 0.6500 \\
$a_{7}$ & & & -.0293 & 0.0327 & 0.1387 & 0.1929 \\
$\lambda_{8}$ & & & & 0.9401 & 0.8469 & 0.7570 \\
$a_{8}$ & & & & -.0252 & 0.0040 & 0.0908 \\
$\lambda_{9}$ & & & & & 0.9494 & 0.8707 \\
$a_{9}$ & & & & & -.0207 & -.0105 \\
$\lambda_{10}$ & & & & & & 0.9566 \\
$a_{10}$ & & & & & & -.0168 \\
$K_{1}$ & 0.5676 & 0.5766 & 0.5817 & 0.5851 & 0.5877 & 0.5897 \\
$K_{2}$ & 0.7007 & 0.7216 & 0.7390 & 0.7523 & 0.7615 & 0.7680 \\
$K_{3}$ & 0.0000 & 0.0000 & 0.0000 & 0.0000 & 0.0000 & 0.0000 \\
$\Delta$ & 0.0727 & 0.0797 & 0.0846 & 0.0882 & 0.0908 & 0.0929
\end{tabular}


Table A.3: Optimal Spacing the regression and scale parameters along with the coefficeints for the ABLUE of $t$-distribution with $p=3$.

\begin{tabular}{|c|c|c|c|c|c|c|}
\hline \multirow[b]{2}{*}{$\boldsymbol{k}$} & \multicolumn{5}{|c|}{ D.F. $=3$} & \multirow[b]{2}{*}{10} \\
\hline & 5 & 6 & 7 & 8 & 9 & \\
\hline $\begin{array}{l}\lambda_{1} \\
a_{1}\end{array}$ & $\begin{array}{l}0.1165 \\
0.0182\end{array}$ & $\begin{array}{l}0.0872 \\
-.0057\end{array}$ & $\begin{array}{l}0.0674 \\
-.0139\end{array}$ & $\begin{array}{l}0.0538 \\
-.0157\end{array}$ & $\begin{array}{l}0.0440 \\
-.0151\end{array}$ & $\begin{array}{l}0.03685 \\
-.0136\end{array}$ \\
\hline $\begin{array}{l}\lambda_{2} \\
a_{2}\end{array}$ & $\begin{array}{l}0.3047 \\
0.2809\end{array}$ & $\begin{array}{l}0.2457 \\
0.1779\end{array}$ & $\begin{array}{l}0.2011 \\
0.1083\end{array}$ & $\begin{array}{l}0.1668 \\
0.0629\end{array}$ & $\begin{array}{l}0.139 \mathrm{~S} \\
0.0339\end{array}$ & $\begin{array}{l}0.115 .8 \\
0.0155\end{array}$ \\
\hline $\begin{array}{l}\lambda_{3} \\
a_{3}\end{array}$ & $\begin{array}{l}0.5000 \\
0.4017\end{array}$ & $\begin{array}{l}0.4150 \\
0.3277\end{array}$ & $\begin{array}{l}0.3498 \\
0.2528\end{array}$ & $\begin{array}{l}0.2985 \\
0.1896\end{array}$ & $\begin{array}{l}0.2531 \\
0.1349 .4\end{array}$ & $\begin{array}{l}0.22: 31) \\
0.1(010 \times\end{array}$ \\
\hline $\begin{array}{l}\lambda_{4} \\
a_{4}\end{array}$ & $\begin{array}{l}0.6953 \\
0.2809\end{array}$ & $\begin{array}{l}0.2850 \\
0.327 i\end{array}$ & $\begin{array}{l}0.5000 \\
0.305 i\end{array}$ & $\begin{array}{l}0.4327 \\
0.2633\end{array}$ & $\begin{array}{l}0.3783 \\
0.2186\end{array}$ & $\begin{array}{l}0.33333 \\
0.1774\end{array}$ \\
\hline $\begin{array}{l}\lambda_{5} \\
a_{5}\end{array}$ & $\begin{array}{l}0.8835 \\
0.0182\end{array}$ & $\begin{array}{l}0.7543 \\
0.1779\end{array}$ & $\begin{array}{l}0.6502 \\
0.2528\end{array}$ & $\begin{array}{l}0.5673 \\
0.26333\end{array}$ & $\begin{array}{l}0.5000 \\
0.246 \pm 2\end{array}$ & $\begin{array}{l}0.44 .14 \\
0.2192\end{array}$ \\
\hline $\begin{array}{l}\lambda_{6} \\
a_{6}\end{array}$ & & $\begin{array}{c}0.9128 \\
-.0057\end{array}$ & $\begin{array}{l}0.7989 \\
0.1083\end{array}$ & $\begin{array}{l}0.7015 \\
0.1896\end{array}$ & $\begin{array}{l}0.6217 \\
0.2186\end{array}$ & $\begin{array}{l}0.55 i n d \\
0.2192\end{array}$ \\
\hline $\begin{array}{l}\lambda_{7} \\
a_{7}\end{array}$ & & & $\begin{array}{l}0.9326 \\
-.0139\end{array}$ & $\begin{array}{l}0.8332 \\
0.0629\end{array}$ & $\begin{array}{l}0.7429 \\
0.1394\end{array}$ & $\begin{array}{l}\text { O.figifit } \\
\text { 0.1779 }\end{array}$ \\
\hline $\begin{array}{l}\lambda_{8} \\
a_{8}\end{array}$ & & & & $\begin{array}{l}0.9462 \\
-.0157\end{array}$ & $\begin{array}{l}0.8602 \\
0.0339\end{array}$ & $\begin{array}{l}0.7769 \\
0.160 \times\end{array}$ \\
\hline $\begin{array}{l}\lambda_{9} \\
a_{9}\end{array}$ & & & & & $\begin{array}{r}0.95600 \\
-.0151\end{array}$ & $\begin{array}{l}0 . \times \times 17 \\
0.0157\end{array}$ \\
\hline $\begin{array}{l}\lambda_{10} \\
a_{10}\end{array}$ & & & & & & $\begin{array}{r}0.96 i 32 \\
-.0136\end{array}$ \\
\hline $\begin{array}{c}K_{1} \\
K_{2} \\
K_{3} \\
\Delta\end{array}$ & $\begin{array}{l}0.6376 \\
0.8599 \\
0.0000 \\
0.1421\end{array}$ & $\begin{array}{l}0.6441 \\
0.8964 \\
0.0000 \\
0.1543\end{array}$ & $\begin{array}{l}0.6489 \\
0.9194 \\
0.0000 \\
0.1630\end{array}$ & $\begin{array}{l}0.6523 \\
0.9354 \\
0.0000 \\
0.1694\end{array}$ & $\begin{array}{l}0.6549 \\
0.9472 \\
0.0000 \\
0.1742\end{array}$ & $\begin{array}{l}0.656(6 x \\
0.9560 \\
0 .(6001) \\
0.1779\end{array}$ \\
\hline
\end{tabular}


Talde: A.4: Optimal Spacing the regression and scale parameters along with the coeffireints for the ABLUE of t-distributic, 1 with $p=3$.

\begin{tabular}{|c|c|c|c|c|c|c|}
\hline \multirow[b]{2}{*}{$\boldsymbol{k}$} & \multicolumn{5}{|c|}{$D . F=5$} & \multirow[b]{2}{*}{10} \\
\hline & 5 & 6 & 7 & 8 & 9 & \\
\hline $\begin{array}{l}\lambda_{1} \\
a_{1}\end{array}$ & $\begin{array}{l}0.0959 \\
0.0522\end{array}$ & $\begin{array}{l}0.0734 \\
0.0244\end{array}$ & $\begin{array}{l}0.0576 \\
0.0100\end{array}$ & $\begin{array}{l}0.0461 \\
0.0025\end{array}$ & $\begin{array}{r}0.0376 \\
-.0014\end{array}$ & $\begin{array}{c}0.0312 \\
-.0034\end{array}$ \\
\hline $\begin{array}{l}\lambda_{2} \\
a_{2}\end{array}$ & $\begin{array}{l}0.2786 \\
0.2638\end{array}$ & $\begin{array}{l}0.2190 \\
0.1763\end{array}$ & $\begin{array}{l}0.1760 \\
0.1183\end{array}$ & $\begin{array}{l}0.1441 \\
0.0798\end{array}$ & $\begin{array}{l}0.1198 \\
0.0539\end{array}$ & $\begin{array}{l}0.100 \\
0.036 .4\end{array}$ \\
\hline $\begin{array}{l}\lambda_{3} \\
a_{3}\end{array}$ & $\begin{array}{l}0.5000 \\
0.3682\end{array}$ & $\begin{array}{l}0.4025 \\
0.2993\end{array}$ & $\begin{array}{l}\mathbf{0 . 3 3 0 1} \\
\mathbf{0 . 2 3 2 5}\end{array}$ & $\begin{array}{l}0.2749 \\
0.1777\end{array}$ & $\begin{array}{l}0.2320 \\
0.1352\end{array}$ & $\begin{array}{l}0.1980 \\
0.1027\end{array}$ \\
\hline $\begin{array}{l}\lambda_{1} \\
a_{4}\end{array}$ & $\begin{array}{l}0.7214 \\
0.2638\end{array}$ & $\begin{array}{l}0.5975 \\
0.2993\end{array}$ & $\begin{array}{l}0.5000 \\
0.2783\end{array}$ & $\begin{array}{l}0.4232 \\
0.2400\end{array}$ & $\begin{array}{l}0.3620 \\
0.2002\end{array}$ & $\begin{array}{l}0.3126 \\
0.1643\end{array}$ \\
\hline $\begin{array}{l}\lambda_{5} \\
a_{5}\end{array}$ & $\begin{array}{l}0.9041 \\
0.0522\end{array}$ & $\begin{array}{l}0.7810 \\
0.1763\end{array}$ & $\begin{array}{l}0.6699 \\
0.2325\end{array}$ & $\begin{array}{l}0.5768 \\
0.2400\end{array}$ & $\begin{array}{l}0.5000 \\
0.22 .43\end{array}$ & $\begin{array}{l}0.4365 \\
0.1999\end{array}$ \\
\hline $\begin{array}{l}\lambda_{6} \\
a_{6}\end{array}$ & & $\begin{array}{l}0.9266 \\
0.0244\end{array}$ & $\begin{array}{l}0.8240 \\
0.1183\end{array}$ & $\begin{array}{l}0.7251 \\
0.1777\end{array}$ & $\begin{array}{l}0.6380 \\
0.2002\end{array}$ & $\begin{array}{l}0.5635 \\
0.1999\end{array}$ \\
\hline $\begin{array}{l}\lambda_{i} \\
a_{i}\end{array}$ & & & $\begin{array}{l}0.9424 \\
0.0100\end{array}$ & $\begin{array}{l}0.8559 \\
0.0798\end{array}$ & $\begin{array}{l}0.7680 \\
0.1352\end{array}$ & $\begin{array}{l}0.6874 \\
0.16 .13\end{array}$ \\
\hline $\begin{array}{l}\lambda_{8} \\
a_{s}\end{array}$ & & & & $\begin{array}{l}0.9539 \\
0.0025\end{array}$ & $\begin{array}{l}0.8802 \\
0.0539\end{array}$ & $\begin{array}{l}0.8020 \\
0.102 \tau\end{array}$ \\
\hline $\begin{array}{l}\lambda_{y} \\
a_{9}\end{array}$ & & & & & $\begin{array}{r}0.9624 \\
-.0014\end{array}$ & $\begin{array}{l}0.8992 \\
0.0364\end{array}$ \\
\hline $\begin{array}{l}\lambda_{10} \\
a_{10}\end{array}$ & & & & & & $\begin{array}{r}0.9688 \\
-.0034\end{array}$ \\
\hline $\begin{array}{l}\boldsymbol{K}_{1} \\
\boldsymbol{K}_{2} \\
\boldsymbol{h}_{3} \\
\boldsymbol{\Delta}\end{array}$ & $\begin{array}{l}0.7213 \\
1.0465 \\
0.0000 \\
0.2833\end{array}$ & $\begin{array}{l}0.7280 \\
1.0974 \\
0.0000 \\
0.3082\end{array}$ & $\begin{array}{l}0.7326 \\
1.1315 \\
0.0000 \\
0.3259\end{array}$ & $\begin{array}{l}0.7358 \\
1.1555 \\
0.0000 \\
0.3388\end{array}$ & $\begin{array}{l}0.7383 \\
1.1728 \\
0.0000 \\
0.3484\end{array}$ & $\begin{array}{l}0.7401 \\
1.1858 \\
0.0000 \\
0.3558\end{array}$ \\
\hline
\end{tabular}


Table A.5: Optimal Spacing the regression and scale paramelers along with the coefficeints for the ABLLE of $t$-distribution with $p=3$.

\begin{tabular}{|c|c|c|c|c|c|c|}
\hline \multicolumn{7}{|c|}{$D . F=10$} \\
\hline$k$ & 5 & 6 & $i$ & 8 & 9 & 10 \\
\hline$\lambda_{1}$ & 0.0710 & 0.0527 & 0.040 .4 & 0.0319 & 0.0257 & 0.0210 \\
\hline$a_{1}$ & 0.0730 & 0.0444 & 0.0281 & 0.0183 & 0.0122 & (1.00N:3 \\
\hline$\lambda_{2}$ & 0.247 .4 & 0.1856 & 0.1436 & $0.113 !$ & 0.0923 & 0.07itil) \\
\hline$a_{2}$ & 0.2521 & 0.1718 & 0.1197 & 0.085 .3 & $0.06 i 20$ & 0.016010 \\
\hline$\lambda_{3}$ & 0.5000 & 0.3861 & 0.3041 & 0.24 .2 & 0.1995: & (1. 1 (65s) \\
\hline$a_{3}$ & 0.3499 & 0.28 .35 & $0.2: 201$ & $0.16 \times 4$ & 0.130110 & 0.111114 \\
\hline$\lambda_{4}$ & 0.7526 & 0.6139 & 0.5000 & 0.4103 & 0.3402 & $0.2 \times 282$ \\
\hline$a_{4}$ & 0.2521 & 0.2838 & 0.2643 & 0.2275 & 0.18903 & 0.1551 \\
\hline$\lambda_{s}$ & 0.9290 & 0.8141 & 0.6929 & 0.58 .97 & 0.501000 & 0.4254 \\
\hline$a_{:}$ & 0.0730 & 0.1718 & 0.2201 & 0.2275 & $0.21: 29$ & 0.1895 \\
\hline$\lambda_{6}$ & & 0.9473 & 0.8564 & 0.7558 & 0.6598 & 0.5741 \\
\hline$a_{6}$ & & 0.0444 & 0.1197 & 0.1689 & 0.1893 & $0.16 ! 97$ \\
\hline$\lambda_{i}$ & & & 0.9596 & 0.8861 & $0.8000 ;$ & $0.714 x$ \\
\hline$a_{:}$ & & & $0.0 \pm 81$ & 0.0853 & 0.13060 & 0.15 .5 .4 \\
\hline$\lambda_{E}$ & & & & 0.9681 & 0.9077 & 0.5.315 \\
\hline$a_{8}$ & & & & 0.018 .3 & 0.0620 & $0.100 \%$ \\
\hline$\lambda_{9}$ & & & & & 0.9743 & 0.92 .411 \\
\hline$a_{9}$ & & & & & 0.0122 & 0.0 .16010 \\
\hline$\lambda_{10}$ & & & & & & 0.97900 \\
\hline$a_{10}$ & & & & & & $0.00 \times .3$ \\
\hline$K_{1}$ & 0.8092 & 0.8183 & 0.8245 & 0.8287 & 0.8311 & 0.8342 \\
\hline$K_{2}$ & $1.260 \pi$ & 1.3262 & 1.3712 & 1.4034 & 1.4272 & $1.44 \%, 3$ \\
\hline$K_{3}$ & 0.0000 & 0.0000 & 0.0000 & 0.00000 & $0.00001)$ & $0.00000)$ \\
\hline$\Delta$ & 0.5404 & 0.5948 & 0.6335 & 0.66221 & $0.68 \times 3.4$ & (0.689.9) \\
\hline
\end{tabular}


Table: A.6: Optimal Spacing the regression and scale parameters along with the coefficeints for the ABLUE of $t$-distribution with $p=3$.

\begin{tabular}{|c|c|c|c|c|c|c|}
\hline \multicolumn{7}{|c|}{ D.F. $=20$} \\
\hline$k$ & 5 & 6 & 7 & 8 & 9 & 10 \\
\hline$\lambda_{1}$ & 0.0569 & 0.0408 & 0.0304 & 0.0233 & 0.0184 & 0.014 ? \\
\hline$a_{1}$ & 0.0821 & 0.0530 & 0.0359 & 0.0252 & 0.0183 & 0.0136 \\
\hline$\lambda_{2}$ & 0.2271 & 0.1642 & 0.1230 & 0.0948 & 0.0748 & 0.0602 \\
\hline$a_{2}$ & 0.2469 & 0.1697 & 0.1199 & 0.0870 & 0.0648 & 0.0493 \\
\hline$\lambda_{3}$ & 0.5000 & 0.3744 & 0.2859 & 0.2229 & 0.1773 & 0.1434 \\
\hline$a_{3}$ & 0.3419 & 0.2773 & $0 .: 148$ & 0.1650 & 0.1275 & 0.0996 \\
\hline$\lambda_{4}$ & 0.7729 & 0.6256 & 0.5000 & 0.4007 & 0.3242 & 0.2654 \\
\hline$a_{4}$ & 0.2469 & 0.2773 & 0.2589 & 0.2227 & 0.1849 & 0.1516 \\
\hline$\lambda_{5}$ & 0.9431 & 0.8358 & 0.7141 & 0.5993 & 0.5000 & 0.4178 \\
\hline$a_{3}$ & 0.0821 & 0.1697 & 0.2148 & $0.222 i$ & 0.2089 & 0.1859 \\
\hline$\lambda_{6}$ & & 0.9592 & 0.8770 & 0.7771 & 0.6758 & 0.5822 \\
\hline$a_{6}$ & & 0.0530 & 0.1199 & 0.1650 & 0.1849 & 0.1859 \\
\hline$\lambda_{i}$ & & & 0.9696 & 0.9052 & $0.822 i$ & 0.7346 \\
\hline$a_{7}$ & & & 0.0359 & 0.0870 & 0.1275 & 01516 \\
\hline$\lambda_{k}$ & & & & 0.9767 & 0.9252 & 0.8566 \\
\hline$a_{\mathrm{g}}$ & & & & 0.0252 & 0.0648 & 0.0996 \\
\hline$\lambda_{y}$ & & & & & 0.9816 & 0.9398 \\
\hline$a_{9}$ & & & & & 0.0183 & 0.0493 \\
\hline$\lambda_{10}$ & & & & & & 0.9853 \\
\hline$a_{10}$ & & & & & & 0.0136 \\
\hline$k_{1}$ & 0.8661 & 0.8777 & 0.8854 & 0.8909 & 0.8948 & 0.8978 \\
\hline $\boldsymbol{h}_{2}$ & 1.4069 & 1.4832 & 1.5362 & 1.5744 & 1.6028 & 1.6244 \\
\hline$h_{3}$ & 0.0000 & 0.0000 & 0.0000 & 0.0000 & 0.0000 & 0.0000 \\
\hline$\Delta$ & 0.7915 & 0.8800 & 0.9441 & 0.9916 & 1.0276 & 1.0555 \\
\hline
\end{tabular}


Table A.7: Optimal Spacing the regression and scale parameters along with the coefficeints for the ABLUE of $t$-distribution with $p=3$.

\begin{tabular}{|c|c|c|c|c|c|c|}
\hline \multicolumn{7}{|c|}{$D \cdot F .=500$} \\
\hline$k$ & 5 & 6 & 7 & 8 & 9 & 10 \\
\hline$\lambda_{1}$ & 0.0433 & 0.0296 & 0.0211 & 0.0156 & 0.0118 & 0.000 .1 \\
\hline$a_{1}$ & 0.0903 & .0605 & .0425 & 0.0311 & 0.023 .4 & 0.0180 \\
\hline$\lambda_{2}$ & 0.2041 & 0.1407 & 0.1009 & 0.0748 & 0.0 .569 & 0.0 .1 .13 \\
\hline$a_{2}$ & 0.2425 & 0.1681 & 0.1203 & 0.088 & 0.0672 & 0.0521 \\
\hline$\lambda_{3}$ & 0.5000 & 0.3602 & 0.2642 & 0.1982 & 0.1521 & 0.1190 \\
\hline$a_{3}$ & 0.3344 & 0.2715 & 0.2100 & 0.1615 & 0.1253 & 0.098 \\
\hline$\lambda_{4}$ & 0.7959 & 0.6398 & 0.5000 & 0.3888 & 0.30 .16 & 0.2 .116 \\
\hline$a_{4}$ & 0.2425 & 0.2715 & 0.2543 & 0.2187 & 0.1812 & 0.148 .3 \\
\hline$\lambda_{5}$ & 0.9567 & 0.8593 & 0.7358 & 0.6112 & 0.5000 & 0.4076 \\
\hline$a_{5}$ & 0.0903 & 0.1681 & 0.2100 & 0.2187 & 0.2058 & $0.1 \times 30$ \\
\hline$\lambda_{6}$ & & 0.9704 & 0.8991 & 0.8018 & 0.6954 & $0.59 \div 24$ \\
\hline$a_{6}$ & & 0.0605 & 0.1203 & $0 . i+15$ & 0.1812 & 0.1830 \\
\hline$\lambda_{r}$ & & & 0.9789 & $0.925 \%$ & 0.8479 & $0.75 \times 4$ \\
\hline$a_{7}$ & & & 0.0425 & 0.0887 & 0.125 .3 & 0.148 .3 \\
\hline$\lambda_{8}$ & & & & 0.9844 & 0.9431 & $0.8 \times 10$ \\
\hline$a_{5 s}$ & & & & 0.0311 & 0.0672 & 0.09855 \\
\hline$\dot{\lambda}_{9}$ & & & & & 0.9882 & 0.95557 \\
\hline$a_{9}$ & & & & & 0.0234 & 0.0521 \\
\hline$\lambda_{10}$ & & & & & & 0.9308 \\
\hline$a_{10}$ & & & & & & 0.0180 \\
\hline$K_{1}$ & 0.9330 & 0.9481 & 0.9584 & 0.9656 & 0.9710 & 0.9750 \\
\hline$K_{2}$ & 1.5834 & 1.6739 & 1.7374 & 1.7835 & 1.8180 & 1.8445 \\
\hline$K_{3}$ & 0.0000 & 0.0000 & 0.0000 & 0.0000 & 0.0000 & 0.00000 \\
\hline$\Delta$ & 1.1999 & 1.3528 & 1.4657 & 1.5507 & 1.6159 & $1.66 ; 6 ; 9$ \\
\hline
\end{tabular}




\section{Appendix A}

\section{Logistic Distribution Table}


Table A.1: Optimal Spacing for the regression and scale parameters along with the coefficients for the ABLUE of Logistic distribution when $\Delta$ is maximized

\begin{tabular}{|c|c|c|c|c|c|c|c|c|c|}
\hline$k$ & 2 & 3 & 4 & 5 & 6 & 7 & 8 & 9 & 10 \\
\hline $\begin{array}{l}\lambda_{1} \\
a_{1} \\
b_{1}\end{array}$ & $\begin{array}{r}\mathbf{0 . 1 8 7 3} \\
\mathbf{0 . 5 0 0 0} \\
\cdot \quad .3407\end{array}$ & $\begin{array}{c}0.1213 \\
0.1801 \\
-.2526\end{array}$ & $\begin{array}{c}0.0752 \\
0.0686 \\
-.1424\end{array}$ & $\begin{array}{l}0.0517 \\
0.0326 \\
-.0929\end{array}$ & $\begin{array}{r}0.0363 \\
0.0164 \\
-.0619\end{array}$ & $\begin{array}{r}0.0265 \\
0.0089 \\
-.0437\end{array}$ & $\begin{array}{l}0.01999 \\
0.0031 \\
-.031 \text { ' }\end{array}$ & $\begin{array}{l}0.01531 \\
0.0103: 31 \\
-.0233 ! 1\end{array}$ & $\begin{array}{l}(1.012010 \\
0 .(0014 \\
-.0184\end{array}$ \\
\hline $\begin{array}{l}\lambda_{2} \\
a_{2} \\
b_{2}\end{array}$ & $\begin{array}{l}0.8 i 27 \\
0.5000 \\
0.3407\end{array}$ & $\begin{array}{l}0.5000 \\
0.6397 \\
0.0000\end{array}$ & $\begin{array}{l}0.3001 \\
0.4314 \\
-.1686\end{array}$ & $\begin{array}{l}0.2086 \\
0.2355 \\
-.1724\end{array}$ & $\begin{array}{l}0.1490 \\
0.1271 \\
-.1370\end{array}$ & $\begin{array}{l}0.1112 \\
0.0734 \\
-.105 .4\end{array}$ & $\begin{array}{l}0.0 \times 19 \\
0.041 .2 \\
-.0809\end{array}$ & $\begin{array}{l}0.06(6) 3 \\
0.0277 \\
-.06(299\end{array}$ & $\begin{array}{l}0.05: 7 \\
0.017 ! \\
-.0196\end{array}$ \\
\hline $\begin{array}{l}\lambda_{3} \\
a_{3} \\
b_{3}\end{array}$ & & $\begin{array}{l}0.8787 \\
0.1801 \\
0.2526\end{array}$ & $\begin{array}{l}0.6999 \\
0.4314 \\
0.1686\end{array}$ & $\begin{array}{l}0.5000 \\
0.4638 \\
0.0000\end{array}$ & $\begin{array}{l}0.3554 \\
0.3566 \\
-.0977\end{array}$ & $\begin{array}{l}0.2657 \\
0.2357 \\
-.1213\end{array}$ & $\begin{array}{l}0.21142 \\
0.1511 \\
-.11: 2\end{array}$ & $\begin{array}{l}0.16(1) ! \\
0.0 ! 1 \times ! \\
-.097 .1\end{array}$ & 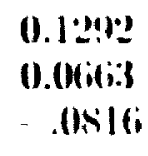 \\
\hline $\begin{array}{l}\lambda_{4} \\
a_{4} \\
b_{4}\end{array}$ & & & $\begin{array}{l}0.9248 \\
0.0686 \\
0.1424\end{array}$ & $\begin{array}{l}0.7914 \\
0.2355 \\
0.1724\end{array}$ & $\begin{array}{l}0.6446 \\
0.3566 \\
0.0977\end{array}$ & $\begin{array}{l}0.5000 \\
0.36: 39 \\
0.0000\end{array}$ & $\begin{array}{l}0.386(33 \\
0.29996 \\
-.06(3)\end{array}$ & $\begin{array}{l}0.305010 \\
0.2201 \\
-.0 \times 599\end{array}$ & $\begin{array}{l}0.2155 \\
0.1568 \\
-.0915\end{array}$ \\
\hline $\begin{array}{l}\lambda_{5} \\
a_{5} \\
b_{5}\end{array}$ & & & & $\begin{array}{l}0.9483 \\
0.0326 \\
0.0929\end{array}$ & $\begin{array}{l}0.8510 \\
0.1271 \\
0.1370\end{array}$ & $\begin{array}{l}0.7343 \\
0.2357 \\
0.1213\end{array}$ & $\begin{array}{l}0.6137 \\
0.2996 \\
0.06: 39\end{array}$ & $\begin{array}{l}0.50000 \\
0.29993 \\
0.010000\end{array}$ & $\begin{array}{l}0.40601 \\
0.2570 \\
-.01501\end{array}$ \\
\hline $\begin{array}{l}\lambda_{6} \\
a_{6} \\
b_{6}\end{array}$ & & & & & $\begin{array}{l}0.9637 \\
0.0164 \\
0.0619\end{array}$ & $\begin{array}{l}0.8888 \\
0.0734 \\
0.1051\end{array}$ & $\begin{array}{l}0.7958 \\
0.1511 \\
0.1132\end{array}$ & $\begin{array}{l}0.6950 \\
0.20206 \\
0.0 \times 49\end{array}$ & $\begin{array}{l}0.5910 \\
0.257010 \\
0.0 .150\end{array}$ \\
\hline $\begin{array}{l}\lambda_{7} \\
a_{7} \\
b_{7}\end{array}$ & & & & & & $\begin{array}{l}0.97355 \\
0.0069 \\
0.0437\end{array}$ & $\begin{array}{l}0.9151 \\
0.0 .142 \\
0.0809\end{array}$ & $\begin{array}{l}0 . \times 3911 \\
0.09989 \\
0.09974\end{array}$ & $\begin{array}{l}0.7515 \\
0.1506 \\
0.0515\end{array}$ \\
\hline $\begin{array}{l}\lambda_{8} \\
a_{8} \\
b_{8}\end{array}$ & & & & & & & $\begin{array}{l}0.9801 \\
0.0051 \\
0.0318\end{array}$ & $\begin{array}{l}0.93337 \\
0.0277 \\
0.0632 !\end{array}$ & $\begin{array}{l}0.8708 \\
0.06660 .3 \\
0.0 \times 16\end{array}$ \\
\hline $\begin{array}{l}\lambda_{9} \\
a_{9} \\
b_{9}\end{array}$ & & & & & & & & $\begin{array}{l}0.9 \times 17 \\
0.00(31 \\
0.0239\end{array}$ & $\begin{array}{l}0.9473 \\
0.0179 \\
0.0436\end{array}$ \\
\hline $\begin{array}{l}\lambda_{10} \\
a_{10} \\
b_{10}\end{array}$ & & & & & & & & & $\begin{array}{l}0.019 \times 010 \\
0.0019 \\
0.0181\end{array}$ \\
\hline $\begin{array}{c}K_{1} \\
K_{2} \\
K_{3} \\
\Delta\end{array}$ & $\begin{array}{l}0.2474 \\
0.8521 \\
0.0000 \\
0.2108\end{array}$ & $\begin{array}{l}0.2959 \\
0.9697 \\
0.0000 \\
0.2870\end{array}$ & $\begin{array}{l}0.3042 \\
1.1267 \\
0.0000 \\
0.3427\end{array}$ & $\begin{array}{l}0.3142 \\
1.1961 \\
0.0000 \\
0.3758\end{array}$ & $\begin{array}{l}0.3184 \\
1.2518 \\
0.0000 \\
0.3986\end{array}$ & $\begin{array}{l}0.3219 \\
1.2874 \\
0.0000 \\
0.4144\end{array}$ & $\begin{array}{l}0.3241 \\
1.3143 \\
0.0000 \\
0.4259\end{array}$ & $\begin{array}{l}0.3257 \\
1.3339 \\
0.00600 \\
0.4345\end{array}$ & $\begin{array}{l}0.3270 \\
1.3490 \\
0.000(1) \\
0.1411\end{array}$ \\
\hline
\end{tabular}


Table A.2: Optimal Spacing for the regression and scale parameters along with the cuefficients for the ABLUE of Logistic distribution when $K_{1} \Delta$ is maximized

\begin{tabular}{|c|c|c|c|c|c|c|c|c|}
\hline$k$ & 3 & 4 & 5 & 6 & 7 & 8 & 9 & 10 \\
\hline $\begin{array}{l}\lambda_{1} \\
a_{1} \\
b_{1}\end{array}$ & $\begin{array}{r}0.1381 \\
0.1984 \\
-.2731\end{array}$ & $\begin{array}{c}0.0895 \\
0.0873 \\
-.1680\end{array}$ & $\begin{array}{c}0.0611 \\
0.0424 \\
-.1092\end{array}$ & $\begin{array}{r}0.0433 \\
0.0222 \\
-.0743\end{array}$ & $\begin{array}{r}0.0317 \\
0.0123 \\
-.0526\end{array}$ & $\begin{array}{c}0.0239 \\
0.0071 \\
-.0386\end{array}$ & $\begin{array}{l}0.0184 \\
0.0043 \\
-.0290\end{array}$ & $\begin{array}{l}0.0144 \\
0.0027 \\
-.0224\end{array}$ \\
\hline $\begin{array}{l}\lambda_{2} \\
a_{2} \\
b_{2}\end{array}$ & $\begin{array}{l}0.5000 \\
0.6032 \\
0.0000\end{array}$ & $\begin{array}{l}0.3331 \\
0.4127 \\
-.1587\end{array}$ & & $\begin{array}{r}0.1718 \\
0.1486 \\
-.1452\end{array}$ & & & $\begin{array}{l}0.0784 \\
0.0366 \\
-.0730\end{array}$ & $\begin{array}{l}0.0626 \\
0.0242 \\
-.0583\end{array}$ \\
\hline $\begin{array}{l}\lambda_{3} \\
a_{3} \\
b_{3}\end{array}$ & $\begin{array}{l}0.8619 \\
0.1984 \\
0.2731\end{array}$ & $\begin{array}{l}0.6669 \\
0.4127 \\
0.158 i\end{array}$ & & $\begin{array}{l}0.3787 \\
0.3293 \\
-.0840\end{array}$ & & $\begin{array}{l}0.2302 \\
0.1656 \\
-.1110\end{array}$ & $\begin{array}{l}0.18 .11 \\
0.1155 \\
-.1008\end{array}$ & $\begin{array}{l}0.1495 \\
0.0811 \\
-.0879\end{array}$ \\
\hline $\begin{array}{l}\lambda_{4} \\
a_{4} \\
b_{4}\end{array}$ & & $\begin{array}{l}0.9105 \\
0.0873 \\
0.1680\end{array}$ & & $\begin{array}{l}0.6213 \\
0.3293 \\
0.084 C\end{array}$ & & $\begin{array}{l}0.4041 \\
0.2704 \\
-.0522\end{array}$ & $\begin{array}{l}0.3297 \\
0.2134 \\
-.0769\end{array}$ & $\begin{array}{r}0.2719 \\
0.1636 \\
-.0846\end{array}$ \\
\hline $\begin{array}{l}\lambda_{5} \\
a_{5} \\
b_{5}\end{array}$ & & & & & & $\begin{array}{l}0.5959 \\
0.2704 \\
0.0522\end{array}$ & $\begin{array}{l}0.5000 \\
0.2603 \\
0.0000\end{array}$ & $\begin{array}{l}0.4206 \\
0.228 . \\
-.0357\end{array}$ \\
\hline $\begin{array}{l}\lambda_{6} \\
a_{6} \\
b_{6}\end{array}$ & & & & $\begin{array}{l}0.9567 \\
0.0222 \\
0.0743\end{array}$ & & $\begin{array}{l}0.7698 \\
0.1656 \\
0.1110\end{array}$ & $\begin{array}{l}0.6703 \\
0.2134 \\
0.0769\end{array}$ & $\begin{array}{l}0.579 .1 \\
0.228 .1 \\
0.03:=\end{array}$ \\
\hline $\begin{array}{l}\lambda_{7} \\
a_{7} \\
b_{i}\end{array}$ & & & & & & $\begin{array}{l}0.9002 \\
0.0569 \\
0.0921\end{array}$ & $\begin{array}{l}0.8159 \\
0.1155 \\
0.1008\end{array}$ & $\begin{array}{l}0.7281 \\
0.1636 \\
0.0846\end{array}$ \\
\hline $\begin{array}{l}\lambda_{8} \\
a_{8} \\
b_{8}\end{array}$ & & & & & & $\begin{array}{l}0.9761 \\
0.0071 \\
0.0386\end{array}$ & $\begin{array}{l}0.9216 \\
0.0366 \\
0.0730\end{array}$ & $\begin{array}{l}0.8505 \\
0.0811 \\
0.0879\end{array}$ \\
\hline $\begin{array}{l}\lambda_{y} \\
a_{9} \\
b_{y}\end{array}$ & & & & & & & $\begin{array}{l}0.9816 \\
0.0043 \\
0.0290\end{array}$ & $\begin{array}{l}0.9374 \\
0.0242 \\
0.0583\end{array}$ \\
\hline $\begin{array}{l}\lambda_{10} \\
a_{10} \\
b_{10}\end{array}$ & & & & & & & & $\begin{array}{l}0.9856 \\
0.0027 \\
0.0224\end{array}$ \\
\hline $\begin{array}{l}\boldsymbol{K}_{1} \\
\boldsymbol{K}_{2} \\
\boldsymbol{K}_{3}\end{array}$ & $\begin{array}{l}0.3000 \\
0.9505 \\
0.0000 \\
0.0855\end{array}$ & $\begin{array}{l}1.0935 \\
0.0000 \\
0.1056\end{array}$ & $\begin{array}{l}0.3173 \\
1.1794 \\
0.0000 \\
0.1187\end{array}$ & $\begin{array}{l}0.3212 \\
1.2366 \\
0.0000 \\
0.1276\end{array}$ & $\begin{array}{l}0.3238 \\
1.2763 \\
0.0000 \\
0.1339\end{array}$ & $\begin{array}{l}0.3257 \\
1.3050 \\
0.0000 \\
0.1384\end{array}$ & $\begin{array}{l}0.3270 \\
1.3263 \\
0.000 n \\
0.14\end{array}$ & $\begin{array}{l}0.3281 \\
1.3426 \\
0.0000 \\
0.1445\end{array}$ \\
\hline
\end{tabular}


Table A.3: Optimal Spacing for the regression and scale parameters along with the coefficients for the ABLUE of Logistic distribution when $h_{1}^{2} \Delta$ is maximized

\begin{tabular}{|c|c|c|c|c|c|c|c|}
\hline$k$ & 4 & 5 & 6 & $\tau$ & 8 & 9 & 10 \\
\hline $\begin{array}{l}\lambda_{1} \\
a_{1} \\
b_{1}\end{array}$ & $\begin{array}{c}0.1000 \\
0.0999 \\
-.1842\end{array}$ & $\begin{array}{l}0.0687 \\
0.0505 \\
-.1218\end{array}$ & $\begin{array}{l}0.0490 \\
0.0271 \\
-.0839\end{array}$ & $\begin{array}{c}0.0361 \\
0.0153 \\
-.0599\end{array}$ & $\begin{array}{c}0.0272 \\
0.0090 \\
-.0441\end{array}$ & $\begin{array}{r}0.0210 \\
0.0055 \\
-.0333\end{array}$ & $\begin{array}{c}0.0165 \\
0.0035 \\
-.0258\end{array}$ \\
\hline $\begin{array}{l}\lambda_{2} \\
a_{2} \\
b_{2}\end{array}$ & $\begin{array}{l}0.3475 \\
0.4001 \\
-.1510\end{array}$ & $\begin{array}{l}0.2515 \\
0.2546 \\
-.1675\end{array}$ & $\begin{array}{l}0.1874 \\
0.1605 \\
-.1480\end{array}$ & $\begin{array}{l}0.1430 \\
0.1023 \\
-.1224\end{array}$ & $\begin{array}{l}0.1112 \\
0.0663 \\
-.0992\end{array}$ & $\begin{array}{l}0.0880 \\
0.0138 \\
-.0800\end{array}$ & $\begin{array}{l}0.0707 \\
0.0295 \\
-.06 .15\end{array}$ \\
\hline $\begin{array}{l}\lambda_{3} \\
a_{3} \\
b_{3}\end{array}$ & $\begin{array}{l}0.6525 \\
0.4001 \\
0.1510\end{array}$ & $\begin{array}{l}0.5000 \\
0.3898 \\
0.0000\end{array}$ & $\begin{array}{l}0.3889 \\
0.3124 \\
--.0758\end{array}$ & $\begin{array}{l}0.3073 \\
0.2340 \\
-.103 i\end{array}$ & $\begin{array}{l}0.2462 \\
0.1711 \\
-.1072\end{array}$ & $\begin{array}{l}0.1998 \\
0.1242 \\
-.10017\end{array}$ & $\begin{array}{r}0.16 .10 \\
0 .(090) 3 \\
-.09(1) 3\end{array}$ \\
\hline $\begin{array}{l}\lambda_{4} \\
a_{4} \\
b_{4}\end{array}$ & $\begin{array}{l}0.9000 \\
0.0999 \\
0.1842\end{array}$ & $\begin{array}{l}0.7485 \\
0.2546 \\
0.1675\end{array}$ & $\begin{array}{l}0.6111 \\
0.3124 \\
0.0758\end{array}$ & $\begin{array}{l}0.5000 \\
0.2967 \\
0.0000\end{array}$ & $\begin{array}{l}0.4121 \\
0.2536 \\
-.0460\end{array}$ & $\begin{array}{r}0.3426 \\
0.2063 \\
-.0694\end{array}$ & $\begin{array}{l}0.2870 \\
0.16 i 3 ! \\
-.0785\end{array}$ \\
\hline $\begin{array}{l}\lambda_{5} \\
a_{5} \\
b_{5}\end{array}$ & & $\begin{array}{l}0.9313 \\
0.0505 \\
0.1218\end{array}$ & $\begin{array}{l}0.8126 \\
0.1605 \\
0.1480\end{array}$ & $\begin{array}{l}0.6927 \\
0.2340 \\
0.1034\end{array}$ & $\begin{array}{l}0.5879 \\
0.2536 \\
0.0460\end{array}$ & $\begin{array}{l}0.5000 \\
0.240 .3 \\
0.0000\end{array}$ & $\begin{array}{l}0.4272 \\
0.21 .28 \\
-.0310\end{array}$ \\
\hline $\begin{array}{l}\lambda_{6} \\
a_{6} \\
b_{6}\end{array}$ & & & $\begin{array}{l}0.9510 \\
0.0271 \\
0.0839\end{array}$ & $\begin{array}{l}0.8570 \\
0.1023 \\
0.1224\end{array}$ & $\begin{array}{l}0.7538 \\
0.1711 \\
0.1072\end{array}$ & $\begin{array}{l}0.6574 \\
0.20633 \\
0.06994\end{array}$ & $\begin{array}{l}0.5726 \\
0.21: x \\
0.0310\end{array}$ \\
\hline $\begin{array}{l}\lambda_{7} \\
a_{7} \\
b_{7}\end{array}$ & & & & $\begin{array}{l}0.9639 \\
0.0153 \\
0.0599\end{array}$ & $\begin{array}{l}0.8888 \\
0.0663 \\
0.0992\end{array}$ & $\begin{array}{l}0.80002 \\
0.1242 \\
0.10017\end{array}$ & $\begin{array}{l}0.7130 \\
0.1639 \\
0.07 \times 7\end{array}$ \\
\hline $\begin{array}{l}\lambda_{8} \\
a_{8} \\
b_{8}\end{array}$ & & & & & $\begin{array}{l}0.9728 \\
0.0090 \\
0.0441\end{array}$ & $\begin{array}{l}0.9120 \\
0.0438 \\
0.0800\end{array}$ & $\begin{array}{l}0.8360 \\
0.0903 \\
0.0903\end{array}$ \\
\hline $\begin{array}{l}\lambda_{9} \\
a_{9} \\
b_{9}\end{array}$ & & & & & & $\begin{array}{l}0.9790 \\
0.0055 \\
0.0333\end{array}$ & $\begin{array}{l}0.929 .3 \\
0.0295 \\
0.0648\end{array}$ \\
\hline $\begin{array}{l}\lambda_{10} \\
a_{10} \\
b_{10}\end{array}$ & & & & & & & $\begin{array}{l}0.3 \times 35 \\
0.0035 \\
0.0258\end{array}$ \\
\hline $\begin{array}{c}K_{1} \\
K_{2} \\
K_{3} \\
\Delta\end{array}$ & $\begin{array}{l}0.3131 \\
1.0741 \\
0.0000 \\
0.0330\end{array}$ & $\begin{array}{l}0.3188 \\
1.1653 \\
0.0000 \\
0.0378\end{array}$ & $\begin{array}{l}0.3224 \\
1.2256 \\
0.0000 \\
0.0411\end{array}$ & $\begin{array}{l}0.3248 \\
1.2675 \\
0.0000 \\
0.0434\end{array}$ & $\begin{array}{l}0.3264 \\
1.2978 \\
0.0000 \\
0.0451\end{array}$ & $\begin{array}{l}0.3277 \\
1.320 .3 \\
0.0000 \\
0.0464\end{array}$ & $\begin{array}{l}0.3286 ; \\
1.3375 \\
0.0000 \\
0.0474\end{array}$ \\
\hline
\end{tabular}


Table A.4: Optimal Spacing for the regression and scale parameters along with the coefficients for the ABLUE of Logistic distribution when $K_{1}^{3} \Delta$ is maximized

\begin{tabular}{|c|c|c|c|c|c|c|}
\hline $\boldsymbol{k}$ & 5 & 6 & 7 & 8 & 9 & 10 \\
\hline $\begin{array}{l}\lambda_{1} \\
a_{1} \\
b_{1}\end{array}$ & $\begin{array}{l}0.0754 \\
0.0573 \\
-.1320\end{array}$ & $\begin{array}{l}0.0541 \\
0.0315 \\
-.0919\end{array}$ & $\begin{array}{l}0.0399 \\
0.0181 \\
-.0661\end{array}$ & $\begin{array}{l}0.0302 \\
0.0108 \\
-.0490\end{array}$ & $\begin{array}{l}0.0234 \\
0.0067 \\
-.0371\end{array}$ & $\begin{array}{l}0.0184 \\
0.0043 \\
-.0288\end{array}$ \\
\hline $\begin{array}{l}\lambda_{2} \\
a_{2} \\
b_{2}\end{array}$ & $\begin{array}{l}0.2628 \\
0.2572 \\
-.1640\end{array}$ & $\begin{array}{l}0.1989 \\
0.1682 \\
-.1486\end{array}$ & $\begin{array}{l}0.1536 \\
0.1106 \\
-.1259\end{array}$ & $\begin{array}{l}0.1206 \\
0.0737 \\
-.1040\end{array}$ & $\begin{array}{l}0.0961 \\
0.0497 \\
-.0852\end{array}$ & $\begin{array}{r}0.0776 \\
0.0341 \\
-.0698\end{array}$ \\
\hline $\begin{array}{l}\lambda_{3} \\
a_{3} \\
b_{3}\end{array}$ & $\begin{array}{l}0.5000 \\
0.3709 \\
0.0000\end{array}$ & $\begin{array}{l}0.3950 \\
0.3004 \\
-.0703\end{array}$ & $\begin{array}{l}0.3168 \\
0.2305 \\
-.0975\end{array}$ & $\begin{array}{r}0.2573 \\
0.1733 \\
-.1034\end{array}$ & $\begin{array}{l}0.2113 \\
0.1293 \\
-.0993\end{array}$ & $\begin{array}{l}0.1751 \\
0.0964 \\
-.0910\end{array}$ \\
\hline $\begin{array}{l}\lambda_{4} \\
a_{4} \\
b_{4}\end{array}$ & $\begin{array}{l}0.7372 \\
0.2572 \\
0.1640\end{array}$ & $\begin{array}{l}0.6050 \\
0.3004 \\
0.0703\end{array}$ & $\begin{array}{l}0.5000 \\
0.2815 \\
0.0000\end{array}$ & $\begin{array}{l}0.4170 \\
0.2422 \\
-.0420\end{array}$ & $\begin{array}{l}0.3507 \\
0.2004 \\
-.0643\end{array}$ & $\begin{array}{l}0.2971 \\
0.1626 \\
-.0742\end{array}$ \\
\hline $\begin{array}{l}\lambda_{5} \\
a_{5} \\
b_{5}\end{array}$ & $\begin{array}{l}0.9246 \\
0.0573 \\
0.1320\end{array}$ & $\begin{array}{l}0.8011 \\
0.1682 \\
0.1486\end{array}$ & $\begin{array}{l}0.6832 \\
0.2305 \\
0.0975\end{array}$ & $\begin{array}{l}0.5830 \\
0.2422 \\
0.0420\end{array}$ & $\begin{array}{l}0.5000 \\
0.2276 \\
0.0000\end{array}$ & $\begin{array}{l}0.4312 \\
0.2026 \\
-.0281\end{array}$ \\
\hline $\begin{array}{l}\lambda_{6} \\
a_{6} \\
b_{6}\end{array}$ & & $\begin{array}{l}0.9459 \\
0.0315 \\
0.0919\end{array}$ & $\begin{array}{l}0.8464 \\
0.1106 \\
0.1259\end{array}$ & $\begin{array}{l}0.7427 \\
0.1733 \\
0.1034\end{array}$ & $\begin{array}{l}0.6493 \\
0.2004 \\
0.0643\end{array}$ & $\begin{array}{l}0.5688 \\
0.2026 \\
0.0281\end{array}$ \\
\hline $\begin{array}{l}\lambda_{7} \\
a_{7} \\
b_{7}\end{array}$ & & & $\begin{array}{l}0.9601 \\
0.0181 \\
0.0661\end{array}$ & $\begin{array}{l}0.879 .4 \\
0.0737 \\
0.1040\end{array}$ & $\begin{array}{l}0.7887 \\
0.1293 \\
0.0993\end{array}$ & $\begin{array}{l}0.7029 \\
0.1626 \\
0.0742\end{array}$ \\
\hline $\begin{array}{l}\lambda_{8} \\
a_{8} \\
b_{8}\end{array}$ & & & & $\begin{array}{l}0.9698 \\
0.0108 \\
0.0490\end{array}$ & $\begin{array}{l}0.9039 \\
0.0497 \\
0.0852\end{array}$ & $\begin{array}{l}0.8249 \\
0.0964 \\
0.0910\end{array}$ \\
\hline $\begin{array}{l}\lambda_{9} \\
a_{9} \\
b_{9}\end{array}$ & & & & & $\begin{array}{l}0.9766 \\
0.0067 \\
0.0371\end{array}$ & $\begin{array}{l}0.9224 \\
0.0341 \\
0.0698\end{array}$ \\
\hline $\begin{array}{l}\lambda_{10} \\
a_{10} \\
b_{10}\end{array}$ & & & & & & $\begin{array}{l}0.9816 \\
0.0043 \\
0.0288\end{array}$ \\
\hline $\begin{array}{c}K_{1} \\
K_{2} \\
K_{3} \\
\Delta\end{array}$ & $\begin{array}{l}0.3198 \\
1.1534 \\
0.0000 \\
0.0121\end{array}$ & $\begin{array}{l}0.3231 \\
1.2163 \\
0.0000 \\
0.0133\end{array}$ & $\begin{array}{l}0.3253 \\
1.2600 \\
0.0000 \\
0.0141\end{array}$ & $\begin{array}{l}0.3269 \\
1.2916 \\
0.0000 \\
0.0147\end{array}$ & $\begin{array}{l}0.3280 \\
1.3152 \\
0.0000 \\
0.0152\end{array}$ & $\begin{array}{l}0.3289 \\
1.3332 \\
0.0000 \\
0.0156\end{array}$ \\
\hline
\end{tabular}




\section{Bibliography}

Abdelmalek, N.N. (1974). On the Discreate Linear $L_{1}$ Approximation and $L_{1}$ Solution of over determind Linear Equation. Journal of Approsimation Theory, 11 38-53.

Bahadur, R.R. (1966). A Note on Quantiles in Large Samples. Ann. Mtuth. Statist., 37, 577-580.

Balmer, D.W., Boulton, M. and Sack, A. (1974). Optimal Solutions in Paran!eter Estimation for Cauchy Distribution. JASA, 69, 238-242.

Balakrishnan, N. and Cohen, A.C. (1991). Order Statistics and Infr wurt. Academic Press

Bassett, G. and Koenker,R. (1978). Asymptotic Theory of least Almelute Error Regression. JASA, Vol. 73, No. 363, 618-622.

Berger, J.O., Bock, M.E., Brown, L.D., Casella, G. and (Gleser, L. (1977). Minimax estimation of normal mean vector for artitrary quadratic loss and unknown covariance matrix. Ann. Statist. 5 763-771.

Bickel, P.J. (1968). Some Contributions to the Theory of Order Statistic's. Proceedings of the Fifth Berkeley Symposium on Mathematical Statistu's, Vol. 1, 575-591.

Bickel, P.J. (1973). On Some Analogues to Linear Combinations of (Orders Statistics in the Linear Model. Annals of Statistics, 1, 597-616. 
Brockwell, P.J. and Davis, R.A. (1991). Time Series: Theory and Methods. New York: Springer.

Cane, G.J. (1974). Linear Estimation of Parameters of the Cauchy Distribution Based on Sample Quantiles. JASA, 69, 242-245.

Chernoff, H., Gastwirth, J.L. and Johns, M.V. (1967). Asymptotic Distribution of Linear Combinations of Functions of Order Statistics with Applications to Estimation. Ann. Math. Statist. 38, 52-72.

Csörgö, M. and Révész, P. (1975). Some Notes on the Empirical Distribution Function and the Quantile Process, in Limit Theorem of Probability. Amsterdam: North-Holland Publishing Co., 59-71.

David, H.A. (1981). Order Statistics, 2nd ed. Ncw York, Wilcy.

Ferguson, T.S. (1967). Mathematical Statistics, a Decision Theoretic Approach. Academic Press.

Fuller, W.A (1976). Introduction to Statistical Time Series. Neu York. Miley Gastwirth, J.L. (1966). On Robust Procedures. JASA, 65, 946-973.

Hall, P. and Heyde, C.C. (1980). Martingale Limit Theory and its Application. Academic Press.

Hannan, E.J. (1970). Multiple Time Series. New York, Wiley.

Hodges, J.L., Jr. and Lehmann, E.L. (1963). Estimation of Location based on rank test. Ann. Math. Statist. 34, 598-611.

Hogg, R.V. (1974). Adaptive Robust Procedures: A Partial Review and Some Suggestions for Future Application and Theory. JASA, 69, 909-927.

Huber, P.J. (1981). Robust Statistics. New York, Wiley.

Judge, G.G. and Bock, M.E. (1978). The statistical implications of pre-test and Stein rule estimators in econometrics. North-Holland Publisher, Amsterdam. 
Jung, J. (1955). On Linear Estimates Defined by a Continuous Wright Punction. Ark. Mat., Band 3, Nr. 15, 199-209.

Jurècková, J. (1984). Regression Quantile and Trimmed Leavt Squuares Fistimator Under a General Design. Kybernctika, Vol.20, No.5, 345.35i.

Koenker, R. and Bassett, G. (1978). Regression Quantiles. Economu trica, lul. 46, No. 1 .

Koenker, R. and D'Orey, V. (1987). Algorithm AS 299: (Computing Regressiem Quantiles. J. Roy. Statist. Soc. Ser. C, 36, 383-393.

Koenker, R. and D'Orey, V. (1993). Remark AS R 92. A remark on Algu. rithm AS 229: Computing Dual Regression Quantiles and Regression Rank Scores. J. Roy. Statist. Soc. Ser. C, 43, 410-414.

Koul, H.L. (1992). Weighted Empiricals and Linear Models. Instilult of Malhematical Statistics, Lecture Notes-Monograph Serics, Vol. 21.

Koul, H.L. (1994). Weak Convergence of Randomly Weighted Ihepe."idenut Residual Empiricals With Applications to Autoregression. The Ann. of Statist., Vol, 22, No. 1, 540-562.

Koul, H.L. and Saleh, A.K.Md.E. (1993). R-Estimation of 'T he l'at amuters of Autoregressive [AR(p)] Models. The Annals of Statistics, Vol. 21. No. 1 , 534-551.

Koul, H.L. and Saleh, A.K.Md.E. (1995). Autoregression Quantile and Related Rank-Scores Processes. The Annals of Statistics, Vol. 23, No. 2, (iîu-(i\&!.

Kulldorf, G. (1964). On the Optimum Spacing of Sample Quantiles from a Normal Distribution, Part II. Skand, Aktuarietidskr, 46, 71-87.

Lloyd, E.H. (1952). Least-Squares Estimation of Location and Sc alu- I'atatnuters Using Order Statistics. Biometrica,39. 
Mosteller, F. (1946). On Some Useful Inefficient statistics. Ann.Math. statist., 17, 377-408.

Ogawa, J. (1951). Contributions to the Theory of Systematic Statistics. I.Osaka Math. 74, 105-121.

Parzen, E. (1979). Nonparametric Statistical Data Modeling. JASA, Vol. 74, No. $365,105-131$.

Puri, M.L. and Sen, P.K. (1985). Nonparametric Methods in General Lincar Models. New York: Wiley.

Randles, R.H and Woife, D.A. (1979). Introduction to The Theory of Nonparametric Statistics. New York: Wiley.

Rav, C.K. (1973). Linear Statistical Inference and Its Applications. 2nd ad. Ncw York: Wiley.

Ruppert, D. and Carroll, R.J. (1980). Trimmed Least Squares Estimation in the Linear Model. JASA, Vol. 75, No. 372, 828-838.

Saleh, A.K.Md.E (1967). Determination of the Exact Optimum Order Statistics for Estimating the Parameters of the Exponential distribution from Censored Samples. Technometrics 9, 279-292.

Saleh, A.K.Md.E. (1992). Contributions to Order Statistics. Dept. of Malh. and Stats., Carleton University, ottaura.

Saleh, A.K.Md.E. and Han, C.P. (1990). Shrinkage Estimation in Regression Analysis. Estadistica, 42, 139, 40-63.

Saleh, A.K.Md.E and Sen, P.K. (1978). Nonparametric Estimation of Location Parameter after a Preliminary Test on Regression. Annals of Statistics, 6, 154-168. 
Saleh, A.K.Md.E and Sen, P.K. (1983). Nonparametric Tist of location after a Preliminary Test on Regression in the Multivariate Case. Communicafions in Statistics, Theory and Method, 12(16), 1855-187:

Saleh, A.K.Md.E and Sen, P.K. (1984a). Least Squares and IRank Order l're' liminary Test Estimation in General Multivariate Linear Mondels. I'rorced-

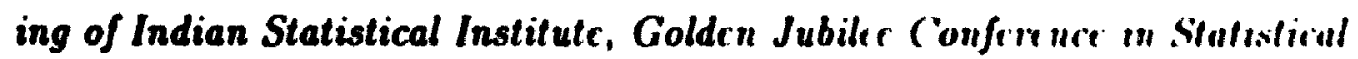
Applications and New Direction-, J.K. Ghosh and J. Roy (tids), 2:BT-2ris.

Saleh, A.K.Md.E and Sen, P.K. (1984b). Nonparametric Preliminary 'Thest In ference. Handbook of Statistics, 4, P.R. Krishnaiah and P.K. S."N (lids), North-Holland, Amsterdam, 275-297.

Saleh, A.K.Md.E and Sen, P.K. (1985a). Nonparametric Shrinkang Histimbiliun in a Parallelism Problem. Sankhya, 47 A, 156-16.5.

Saleh, A.K.Md.E and Sen, P.K. (1985b). On Shrinkage M-estimators of IN cation Parameters. Communications in Statistics, The ary and Mellest. 14(10),2313-2329.

Saleh, A.K.Md.E and Sen, P.K. (1985c). Preliminary Trest Predictedl in (ie.n eral Multivariate Linear Models. Procetdings of the Pacific A re a Situfrolicul Conference, North-Holland Publishers, 619-633x.

Saleh. A.K.Md.E and Sen, P.K. (1985d). Shrinkago Least Squares Fstimation in a Generol Multivariate Linear Model. Proceeding of the Fiffh I'an "om in!" Symposium on Mathematical Statistics, 307-325.

Saleh, A.K.Md.E and Sen, P.K. (1986a). On Shrinkage Last Squat 's listima tion in a Parallelism Problem. Communications in Stutistics, Ihorry and Method, 15(5), 1451-1466.

Saleh, A.K.Md.E and Sen, P.K. (1986b). On Shrinkage K-estimatiom in a Mul tiple Regression Model. Communications in Statistics, Theory and Mc thod. 
15(7), 2229-2244.

Saleh, A.K.Md.E and Shiraishi, T. (1989). On some R-and M-estimators of regression parameters under uncertain restriction. J. Japan Slalist. Sor. Vol. 19 No. 21989 129-137.

Sarhan, A.E. and Greenberg, B.G. (1962). Contributions to Order Statistics. New York: Wiley.

Sclove, S.L., Morris, C. and Radhakrishnan, (1972). Non-optimality of preliminary test estimator of the mean of a multivariate normal distribution. Ann. Statist. 43 1481-1490.

Sen, P.K and Saleh, A.K.Md.E. (198\%). On the preliminary test and shrinkage M-estimation in linear models. Ann. Statist. 15 1580-1592.

Sen, P.K and Singer, J.M. (1993). Large Sample Mtthods in Statisticr. Chapman and Hall.

Stigler, S.M. (1973). Simon Newcomb, Percy Daniell, and the History of Robust Estimation 1885-1920. JASA. 68, 872-79.

Stigler, S.M. (1974). Linear Function of Order Statistics with Smooth Weight Functions. Ann. of Statistics, 2, 676-693.

Stigler, E.M. (1977). Do Robust Estimators Work with Real Data. Annals of Statistics, 5, 1055-1098.

Tukey, J.W. (1960). A Survey Sampling from Contaminated Distribution, Contributions to Probability and Statistics. (I. Olkin, rd.). Stanford ('niv. Press.

Eubank, R.L. (1981a). A Density-Quantile Function Approach to Optimal Spacing Selection. Ann. Statist. 8, 494-500. 
Eubank, R.L. (1981b). Estimation of the Parameters and Quantiles of the Logistic Distribution by Linear Functions of Sample Quantiles. Sirnud. Actuar.J, 229-236. 

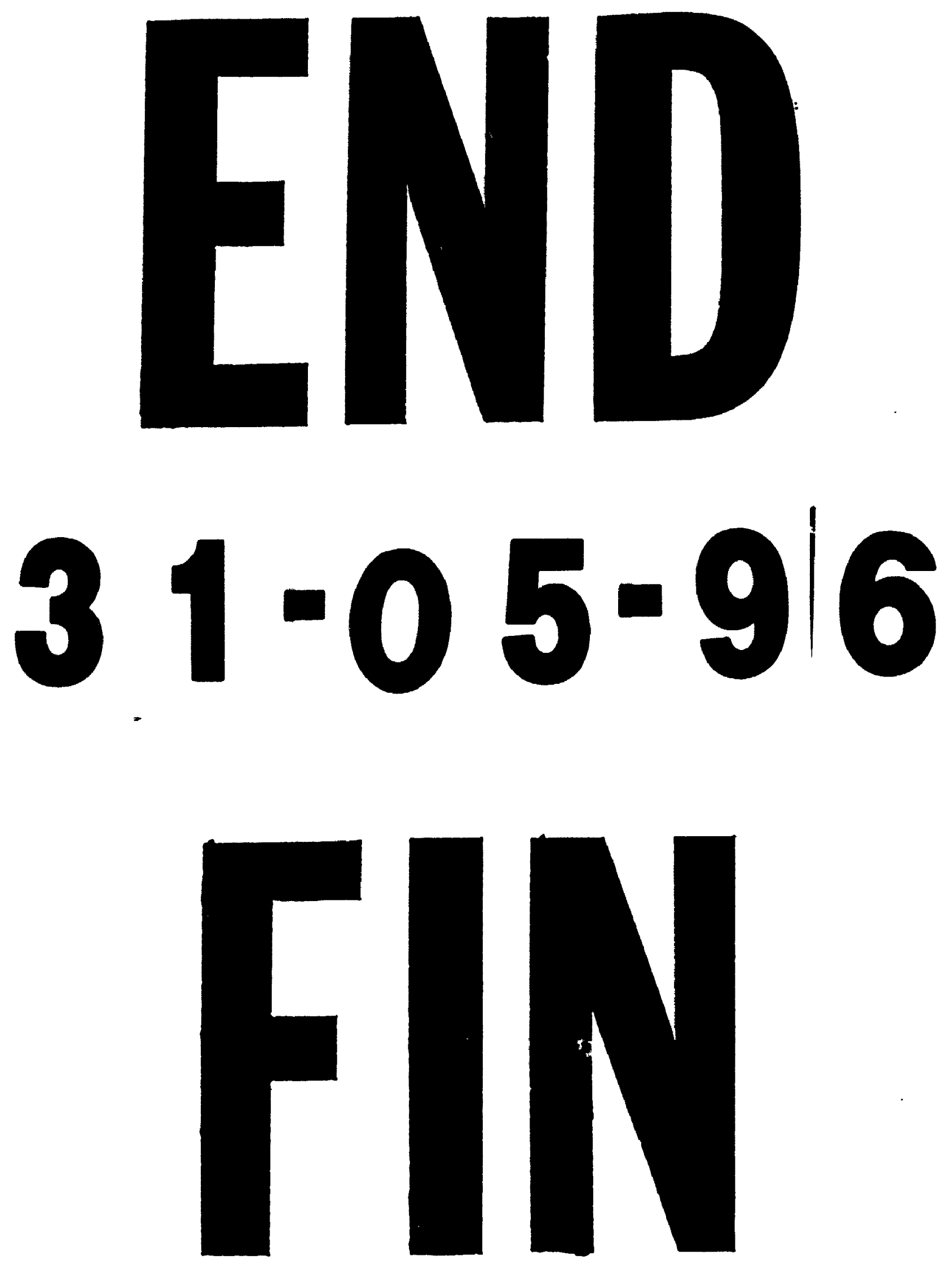